\title{
Preliminary Assessment of Two Alternative Core Design Concepts for the Special Purpose Reactor
}

\author{
James W. Sterbentz \\ James E. Werner \\ Andrew J. Hummel \\ John C. Kennedy \\ Robert C. O'Brien \\ Axel M. Dion \\ Richard N. Wright \\ Krishnan P. Ananth
}

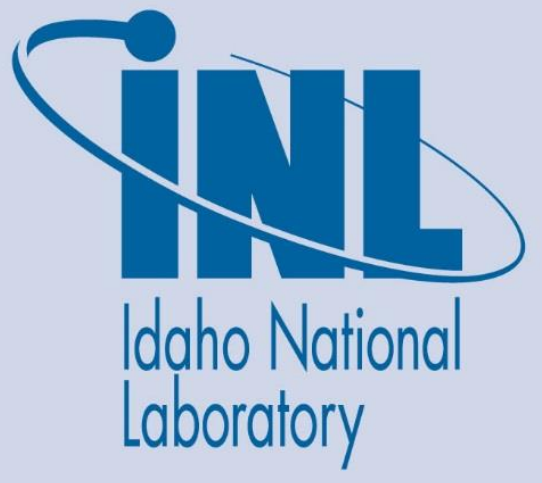

May 2018 


\section{DISCLAIMER}

This information was prepared as an account of work sponsored by an agency of the U.S. Government. Neither the U.S. government nor any agency thereof, nor any of their employees, makes any warranty, expressed or implied, or assumes any legal liability or responsibility for the accuracy, completeness, or usefulness, of any information, apparatus, product, or process disclosed, or represents that its use would not infringe privately owned rights. References herein to any specific commercial product, process, or service by trade name, trade mark, manufacturer, or otherwise, does not necessarily constitute or imply its endorsement, recommendation, or favoring by the U.S. Government or any agency thereof. The views and opinions of authors expressed herein do not necessarily state or reflect those of the U.S. government or any agency thereof. 


\title{
Preliminary Assessment of Two Alternative Core Design Concepts for the Special Purpose Reactor
}

\author{
James W. Sterbentz \\ James E. Werner \\ Andrew J. Hummel \\ John C. Kennedy \\ Robert C. O'Brien \\ Axel M. Dion \\ Richard N. Wright \\ Krishnan P. Ananth
}

May 2018

\author{
Idaho National Laboratory \\ Nuclear Science and Technology Division \\ Idaho Falls, Idaho 83415
}

http://www.inl.gov

Prepared for the

U.S. Department of Energy

Office of Nuclear Energy

Under DOE Idaho Operations Office

Contract DE-AC07-05ID14517 



\begin{abstract}
The Special Purpose Reactor (SPR) is a small $5 \mathrm{MWt}$, heat pipe-cooled, fast reactor based on the Los Alamos National Laboratory (LANL) Mega-Power concept. The LANL concept features a stainless steel monolithic core structure with drilled channels for $\mathrm{UO}_{2}$ pellet stacks and evaporator sections of the heat pipes. Two alternative active core designs are presented here that replace the monolithic core structure with simpler and easier to manufacture fuel elements. The two new core designs are simply referred to as Design A and Design B. In addition to ease of manufacturability, the fuel elements for both Design A and Design B can be individually fabricated, assembled, inspected, tested, and qualified prior to their installation into the reactor core leading to greater reactor system reliability and safety. Design A fuel elements will require the development of a new hexagonally-shaped $\mathrm{UO}_{2}$ fuel pellet. The Design $\mathrm{A}$ configuration will consist of an array of hexagonally-shaped fuel elements with each fuel element having a central heat pipe. This hexagonal fuel element configuration results in four radial gaps or thermal resistances per element. Neither the fuel element development, nor the radial gap issue are deemed to be serious and should not impact an aggressive reactor deployment schedule. Design B uses embedded arrays of heat pipes and fuel pins in a double-wall tank filled with liquid metal sodium. Sodium is used to thermally bond the heat pipes to the fuel pins, but its usage may create reactor transportation and regulatory challenges.

An independent panel of U.S. manufacturing experts has preliminarily assessed the three SPR core designs and views Design A as simplest to manufacture. Herein are the results of a preliminary neutronic, thermal, mechanical, material, and manufacturing assessment of both Design A and Design B along with comparisons to the LANL concept (monolithic core structure). Despite the active core differences, all three reactor concepts behave similarly and retain the same ex-core features and characteristics. While INL has developed Designs A and B, LANL is evaluating a Hot Isostatic Pressed reactor configuration to overcome the drawbacks of the monolithic core design.
\end{abstract}




\section{EXECUTIVE SUMMARY}

The Special Purpose Reactor (SPR) is an innovative small nuclear reactor concept conceived by the Los Alamos National Laboratory (LANL). The heat pipe-cooled, fast-spectrum reactor is designed to be transportable and operate at $5 \mathrm{MWt}$. The active core features an array of liquid metal potassium heat pipes and $\mathrm{UO}_{2}$ fuel pellets embedded in a solid stainless steel monolithic core structure. The heat pipes provide passive cooling without the use of pumps, valves, or primary loop piping, thus avoiding the usual offnormal operating conditions involving loss-of-coolant accident scenarios in commercial reactors. Heat pipe technology is mature and robust, and the large number of in-core heat pipes proposed for the SPR should provide reliable fission heat removal and redundant backup in the event of heat pipe failures. The low reactor power translates into a small reactor footprint, and a self-contained system geometry ideal for small, mobile, plug-and-play power sources for military installations and civilian communities in remote, off-grid locations.

The LANL design concept, also known as the Mega-Power reactor, is mature and well optimized, especially in the areas of neutronics and thermal-hydraulics. The reactor design exhibits many positive characteristics typical of a well-designed reactor core. These include strong negative reactivity feedbacks, low power density, long-life, ample control-drum worth, independent emergency control rod shutdown systems, and passive heat removal via the heat pipes. Reactor kinetic response for this very small fast reactor should be straightforward, predictable, and easy to control with its low-enriched core. In order to maximize electrical output using an open-air Brayton cycle, the active core needs to operate at temperatures as high as possible, in this case approximately $700^{\circ} \mathrm{C}$ which presents material challenges to the in-core stainless steel monolith structure.

The Idaho National Laboratory (INL) recently completed a Phenomena Identification and Ranking Table (PIRT) assessment of the LANL concept. The goal was to identify potential technical and safety issues associated with the concept. Many of the identified issues centered on the stainless steel monolith core structure. In particular the two main issues included: (1) complexity in manufacturing the steel monolith and (2) thermal stresses in the steel under normal operating and failed heat pipe conditions. The steel monolith core requires 3,336 drilled channels to hold $\mathrm{UO}_{2}$ fuel pellets and to act as the in-core evaporator section of the heat pipes. To create the channels in the solid steel monolith, they must be drilled, but deep channel drilling $(1.5 \mathrm{~m})$ with $1 \mathrm{~mm}$ webbing between channels is currently beyond the state of the art for Type 316 stainless steel drilling technology.

Three alternative manufacturing techniques have been proposed for the steel monolith. The first proposed solution is to hot isostatically press (HIP) a stack of pre-drilled plates for a pre-determined amount of time to diffusion bond the plates into a single unit. Each plate would be $2.54 \mathrm{~cm}(1 \mathrm{in}$.) to $30.48 \mathrm{~cm}$ (12 in.) thick with pre-drilled holes (channels) for $\mathrm{UO}_{2}$ pellets and heat pipes. This process avoids deep bore drilling, but does require pre-HIP canning of the exposed plate and channel surfaces, plus post-HIP can removal and channel reaming. Although the HIP process is straightforward, application to a complex object like the proposed monolith structure with a multitude of individual plates and heat pipes, plus other ex-core components will be heavy and difficult to orient, move, and align. The final HIP structure may also prove to be difficult to de-can, inspect, test, repair, and therefore guarantee $100 \%$ bonding of all surfaces and joints. A research and development program will be required to perfect the HIP-core structure and technique. Other possible solutions include powder metallurgy HIP and additive manufacturing; however, these alternative solutions for monolith fabrication will require substantially more research and development effort as well. LANL is currently evaluating these alternative manufacturing approaches.

To avoid the use of a stainless steel monolithic core structure and thereby circumvent the PIRTidentified issues associated with the monolith, INL has proposed two new alternative core design concepts for the Special Purpose Reactor. The two designs are simply referred to as Design A and Design B. The main difference between the three concepts (Design A, Design B, and the LANL concept) is the 
active core geometry and configuration, otherwise all other ex-core features essentially remain the same. In addition, Design A and B adhere to the goals of maintaining the same core size, uranium enrichment, materials, excess reactivity, and performance as the LANL concept. An independent panel of U.S. manufacturing experts assessed the three design concepts and favored Design A for its readiness to manufacture. Design B was viewed as having potential regulatory issues with transport of liquid metal sodium; and alternative manufacturing approaches to the LANL monolith called for further research and development.

In Design A, the active core is composed of an array of hexagonally-shaped fuel elements which are used in place of the LANL steel monolith core structure. Each fuel element features a centrally-located heat pipe surrounded by the hexagonal fuel pellet cladded on both radial sides. Design A fuel pellets will require the development of a new hexagonally-shaped $\mathrm{UO}_{2}$ fuel pellet with a central hole, plus the unique fuel element design will have four radial gaps or thermal resistances per element. But neither the fuel pellet or gas gaps pose a serious design issue. Similarly, Design B uses individual heat pipes and more conventional cladded fuel pins in a sodium-filled double-walled tank. In both Design A and B, the individual heat pipes and fuel elements or fuel pins are readily manufacturable with existing commercial technologies and U.S. vendors. Also, fabrication of these components can be done using production lines to ensure component fabrication, assembly, loading, testing, and inspection consistency, and therefore will provide a high degree of operational reliability for each manufactured component.

In addition to manufacturability, the stainless steel monolith structure exhibits under normal operating conditions high induced thermo-mechanical stresses in the thin webbing between heat pipes and fuel pellet stacks at normal operating temperatures. At temperatures above $575^{\circ} \mathrm{C}$, Type 316 stainless steel is in the high-temperature regime where the physical strength properties began to degrade and ASME code allowable stress levels begin to drop rapidly. The stresses in the monolith steel may be a potential concern down the road, particularly if the steel is deemed to be part of a pressure boundary or load-bearing structure. The two new concepts also use in-core steel for the heat pipe walls and fuel element cladding. Since the core power, core size, heat pipe diameters, and in-core steel wall thicknesses are similar in all three concepts, the maximum steel temperatures are also similar in magnitude. Design A heat-pipe/clad stress levels, however, may prove to be lower than the LANL concept, because of the azimuthal symmetry of the fuel elements and the ability of individual elements to slide axially. Design B clad stress levels are expected to be relatively minor due to the liquid metal sodium bond between fuel pins and heat pipes.

No matter which one of the three active core designs is ultimately selected, the Special Purpose Reactor with its low total core power level of only $5 \mathrm{MWt}$ will allow for the construction of a full-scale, non-nuclear engineering demonstration unit (EDU) that can be heated using electrical heating elements in place of the nuclear fuel. The non-nuclear EDU would be fully instrumented and run at various steadystate power levels and even time-dependent power profiles to simulate reactor transient behavior. The measured data (temperature, stress, strain, pressure, thermal expansion, temperature gradients, and input power) could then be used to establish the reactor system operating parameters and transient characteristics and to validate the computer code models, software, and calculated variable predictions. The EDU could greatly facilitate and accelerate the licensing process. Once the EDU operating characteristics are known and validated, a nuclear-fueled first-of-a-kind (FOAK) reactor system could be built. 


\section{CONTENTS}

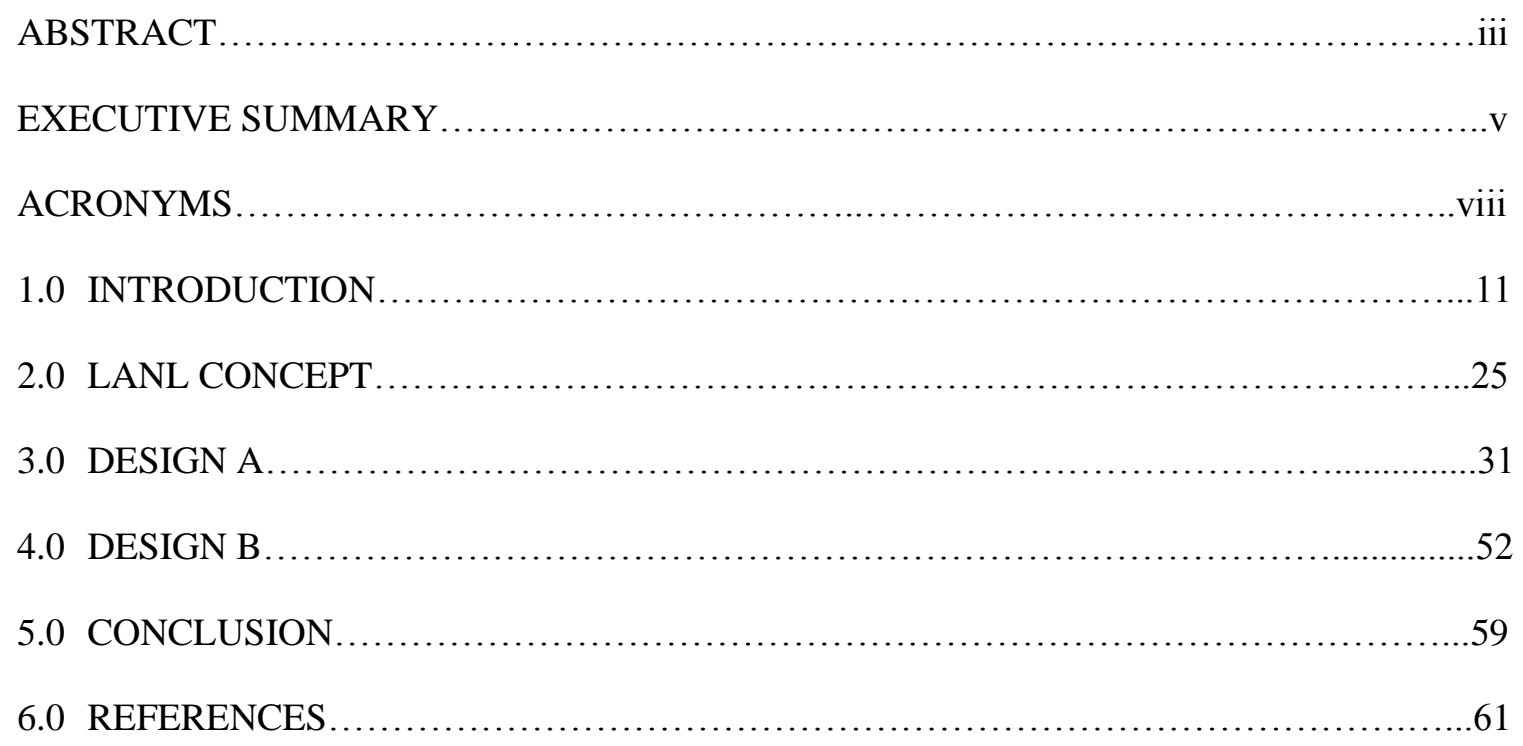

Appendix A: Neutronics Analysis Design A

Appendix B: Neutronics Analysis Design B

Appendix C: Thermal Analysis Design A

Appendix D: Thermal Analysis Design B

Appendix E: Heat Pipe Analysis 


\section{ACRONYMS}

ADU ammonium diuranate

AFC advanced fuel cycle

ASME American Society of Mechanical Engineers

ATC Advanced Cooling Technologies, Inc.

BOL beginning of life

BWXT BWX Technologies, Inc.

CD control drum

CFA Central Facilities Area

CFD computational fluid dynamics

CPP Chemical Processing Plant

DOD Department of Defense

DOE Department of Energy

DPA displacements per atom

EBR Experimental Breeder Reactor

EDM electric discharge machining

EDU engineering demonstration unit

EFF Experimental Fuels Facility

FASB Fuels and Applied Science Building

FCCI fuel-clad chemical interaction

FFTF Fast Flux Test Reactor

FOAK first-of-a-kind

HIP hot isostatic press

HP heat pipe

HPR heat pipe reactor

HX heat exchanger

IAB Industrial Advisory Board

INL Idaho National Laboratory

INTEC Idaho Nuclear Technology and Engineering Center

LANL Los Alamos National Laboratory

LEU low-enriched uranium

LWR light water reactor

MFC Materials and Fuels Complex

MTU metric ton uranium heavy metal 


$\begin{array}{ll}\text { MW } & \text { megawatt } \\ \text { MWD } & \text { megawatt-day } \\ \text { MWe } & \text { megawatt electric } \\ \text { MWt } & \text { megawatt thermal } \\ \text { NNSA } & \text { National Nuclear Security Administration } \\ \text { NRC } & \text { Nuclear Regulatory Commission } \\ \text { PCU } & \text { power conversion unit } \\ \text { PIRT } & \text { phenomena identification and ranking table } \\ \text { PWR } & \text { pressurized water reactor } \\ \text { QA } & \text { quality assurance } \\ \text { RCL } & \text { Radioanalytical Chemistry Laboratory } \\ \text { RSPS } & \text { Radiological Spark Plasma Sintering } \\ \text { SMR } & \text { small modular reactor } \\ \text { SPS } & \text { spark plasma sintering } \\ \text { SPR } & \text { Special Purpose Reactor } \\ \text { SS } & \text { stainless steel } \\ \text { UAC } & \text { ammonium-uranyl-carbonate } \\ \text { ZPPR } & \text { zero power physics reactor }\end{array}$




\section{Preliminary Assessment of Two Alternative Core Design Concepts for the Special Purpose Reactor 1. INTRODUCTION}

\subsection{Micro-Reactor Need}

The U.S. Department of Energy (DOE) and the Department of Defense (DOD) have identified very small modular nuclear reactors (vSMR), or micro-reactors, as a potential means to provide reliable and cost-effective power between 1 and 10 MWe for remote installations. Remote installations could include military operating bases and monitoring stations; perhaps an even larger market demand could come from remote civilian communities and mining operations. Military installations and civilian communities often do not have access to commercial power grids and must rely on fossil fuels or diesel generators to generate their electricity. The cost to generate their electricity becomes exorbitant, not only from the price of the fuel, but also from the transportation costs associated with moving the fuel over long distances and often unpaved roads. Weather conditions, road conditions, and human casualties make electric generation in this manner both costly and unpredictable. Small nuclear reactors may have a role to play here as a steady and reliable source of power.

Nearly $50 \%$ of DOD bases currently require less than 10 MWe and many need only 2 MWe or less. There are approximately 25 critical remote bases, numerous remote mining operations, and multitudes of remote civilian communities that could benefit from a Special Purpose Reactor (SPR) power source. An SPR operating at just 2 MWe could supply the daily electrical needs for 1,550 typical American households or 6,000 people. The SPR could produce electricity for electrical equipment, computers, sensors, communications, diesel generator replacement, and process heat $\left(675^{\circ} \mathrm{C}\right)$ for space heating, chemical reactions, fracking, heavy crude oil cracking, and many other applications. An SPR could also be used to power a large skyscraper or other large facility in an urban setting.

The Defense Science Board (DSB), under the auspices of the DOD, has preliminarily investigated and surveyed the status of micro-reactors currently available to fill the military need for small power sources. The DSB identified two prospective micro-reactor design concepts as its top picks [1][2]. One micro-reactor is the Los Alamos National Laboratory (LANL) heat pipe-cooled, fast reactor concept, also

known as the Mega-Power reactor concept and now as the SPR. This innovative $5 \mathrm{MWt}$ reactor concept is built around a solid stainless steel monolithic core structure with drilled channels for both heat pipes and $\mathrm{UO}_{2}$ fuel pellets [3] [4] [5]. Under steady-state operating conditions, the maximum temperature of the monolith steel core is approximately $700^{\circ} \mathrm{C}$ with maximum $\mathrm{UO}_{2}$ fuel temperatures of around $770^{\circ} \mathrm{C}$ and isothermal heat pipes operating around $675^{\circ} \mathrm{C}$. The ex-core condenser section of the heat pipes will be cooled by forced air convective flow in an open-air recuperated Brayton cycle with an optimal $40.3 \%$ thermal efficiency to generate approximately $2 \mathrm{MWe}$.

The LANL Mega-Power concept was presented to Idaho National Laboratory (INL) by LANL in January 2016 to explore the possibility of an inter-laboratory collaboration effort between the two national laboratories given the role of INL in the DOE, as the lead nuclear energy laboratory and INL's background in reactor development and demonstration. Interest in the concept led to an assessment of the LANL concept using the Phenomena Identification and Ranking Table (PIRT) technique which identified 
both strengths and weaknesses in the design concept. In addition, it led to the INL invention, development, and preliminary assessment of two alternative active core designs for the Special Purpose Reactor, referred to herein simply as Design A and Design B. Based on the preliminary assessments here for Designs A and B, both core designs are viable alternatives to the LANL steel monolith.

\subsection{Manufacturing Capabilities}

During the INL PIRT effort, which was focused on the LANL steel monolith concept, and while the neutronic, thermal, and material analysis of the design was being conducted, several U.S. manufacturing companies (i.e., Westinghouse, PCC York, ATI Metals, and Dearborn Inc.) were contacted to determine the state of the art for bore drilling in a Type 316 stainless steel block. Steel block similar to that which might be used for the LANL monolith structure. After providing company technical experts with drilling specifications for the desired bore channels, it was discovered that the steel monolith could not be fabricated to the desired specifications for single bore channels 1.5 meters in length using current state-ofthe-art drilling technology. An alternative manufacturing technique would be needed.

It was recognized then that industry and stakeholder engagement would be critical in the design, development, and operation of the prototype reactor, both to identify potential collaborators or partners and to ensure early identification of available manufacturing capabilities for timely and affordable reactor development. To facilitate this engagement, INL has, with the help of the DOE-NE Small Modular Reactor (SMR) workshop liaison, reached out to relevant manufacturing experts in industry and academia and assembled an Industrial Advisory Board (IAB). The purpose of IAB was to review the current INL and LANL efforts and critically comment on what the INL-LANL team might have missed or overlooked, as well as to also identify anything in the current approach that could cause concern during manufacturing, system assembly, or deployment.

The IAB is composed of the following members: (1) Jack Lance, DOE/SMR manufacturing workshop liaison, retired nuclear engineer from industry, retired INL employee, and consultant for the energy industry; (2) Nate Ames from Ohio State University, engineering manager and associate director of the Center for Design and Manufacturing Excellence; and (3) David Gandy from the Electric Power Research Institute, technical executive for Nuclear Materials, leader for innovative manufacturing for nuclear power plant components via powder metallurgy and hot isostatic pressing. This group met all day July 20, 2017 with the both the INL and LANL teams together and provided comments and insights related to the manufacturability of the three INL and LANL SPR design concepts.

In advancing Designs A and B, INL considered using an array of Type 316 stainless steel tubes, since they were commercially available in the size of interest and could simplify the core design considerably. Safety was also enhanced because of the ability to clad the fuel pellets and the heat pipes, inspect the tubes, and make assembly easier. These advantages could translate to rapid design and reduced manufacturing cost.

In addition to the IAB panel of manufacturing experts, Professor Mohamed El-Genk, who is an expert from the University of New Mexico on heat pipes, met with the INL team on July 24, 2017. Based on the discussion with Dr. El-Genk, the INL team is confident in the feasibility of using heat pipes for passive cooling of the Special Purpose Reactor. In addition, a manufacturer of heat pipes, Advanced Cooling 
Technologies, Inc. (ATC) was identified, and INL an initiated contact and collaborative effort. LANL has worked with ATC in the past as well. ATC can fabricate custom heat pipes to desired specifications.

\subsection{Stainless Steel}

The LANL concept has several unique design features. One of these unique features is the stainless steel monolith core. Even though stainless steel is a fairly robust structural material with a melting point around $1,510^{\circ} \mathrm{C}$, the steel monolith structure will be operating at elevated temperatures, i.e. in the hightemperature regime of $700-800^{\circ} \mathrm{C}$. The steel monolith core structure is expected to operate under normal steady-state power conditions within a temperature range of $650-720^{\circ} \mathrm{C}$. At these relatively elevated reactor core temperatures, most practical metals suffer some degree of material property change, loss of strength, increased grain growth, migration of elemental constituents, and thermal creep under load. As a result, the number of code-approved metals by the American Society of Mechanical Engineers (ASME) is limited. Type 316 stainless steel is one of the few alloys that has been approved for nuclear applications at temperatures up to $800^{\circ} \mathrm{C}$.

Above $575^{\circ} \mathrm{C}$, the maximum allowable stress values for Type 316 stainless steel alloys fall-off fairly rapidly, and at $700^{\circ} \mathrm{C}$, the maximum allowable stress level is $29.6 \mathrm{MPa}$, a reduction of $70 \%$. Figure 1 shows the maximum allowable stress values for Type 316 stainless steel as a function of temperature per the American Society of Mechanical Engineers (ASME) Section I; Section III, Classes 2 and 3, Section VIII, Division 1; and Section XII (Table 1A). The monolith steel in the LANL concept is expected to operate at a maximum temperature of $721^{\circ} \mathrm{C}$ [4], where the maximum allowable stress is $25.0 \mathrm{MPa}$.

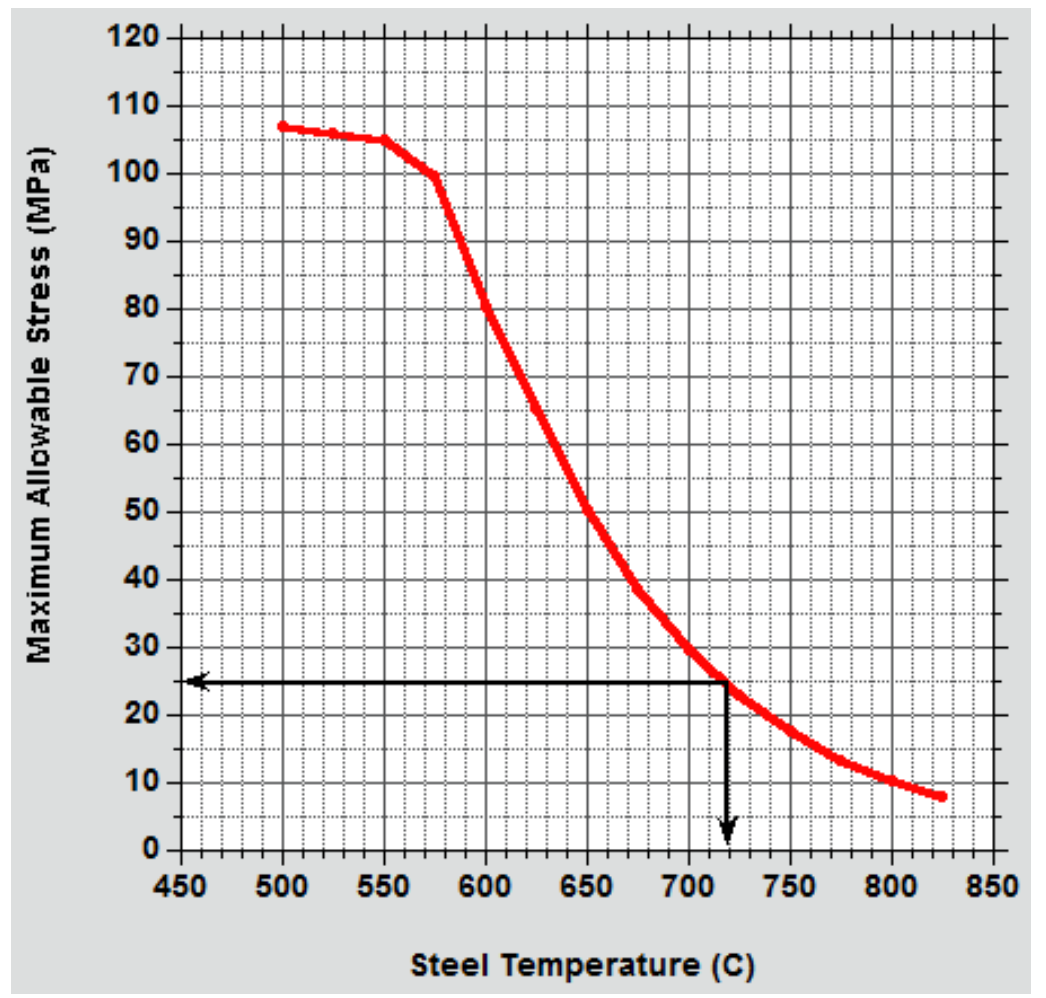

Figure 1. Maximum allowable stress values for Type 316 stainless steel. 
Section III Division 5 of the ASME Boiler and Pressure Vessel Code contains design rules that are applicable for anticipated design conditions for the SPR. This section of the Code also specifies those materials that are allowed for nuclear construction, contains the required material properties for design and construction, specifies acceptable welding processes, and specifies inspection requirements. As previously mentioned, there is only a handful of materials for which sufficient high temperature properties for design are available. These include Type 316 and Type 304 stainless steel and Alloy $800 \mathrm{H}$. These three materials are qualified for Section III, Division 5 in the temperature range anticipated for the Special Purpose Reactor. Time-dependent allowable stresses for Type 316 stainless steel are given for up to 300,000 hours. The design rules can be applied up to $800^{\circ} \mathrm{C}$.

The INL PIRT thermo-mechanical analysis [6] showed that the maximum calculated stress level in the LANL steel monolith under normal reactor operating conditions was approximately $37.1 \mathrm{MPa}$. This maximum stress occurred in the thin $(1.75 \mathrm{~mm}$ thick) monolith steel webbing between two fuel pins. The peak local monolith temperature in this maximum stress region was calculated to be $696^{\circ} \mathrm{C}$. In the ASME code, a temperature of $700^{\circ} \mathrm{C}$ corresponds to a maximum allowable stress of $29.6 \mathrm{MPa}$. The calculated monolith stress at normal operating conditions exceeds this limit by $25 \%$, a potential issue for monolith performance and reactor licensing.

There are several additional factors that could further elevate the stainless steel monolith temperature above the normal operating limit, such as a single or multiple heat pipe failures, core over-power transient, or a loss of heat removal by the power conversion system. Each of these off-normal conditions could potentially push the stress levels further in excess of the allowable limits. An example is a single heat pipe failure [6] which would boost the local maximum stress level to $154.8 \mathrm{MPa}$, a factor of 4 beyond the already exceeded level calculated level at normal operation. It has been argued that the ASME code limits might not apply to the steel monolith, if the monolith is not designated to be a pressure vessel boundary. The final decision would be made by the U.S. Nuclear Regulatory Agency (NRC). For now, the thermal stress issue needs to be further evaluated. Design A attempts to mitigate these stresses and Design B uses a liquid metal sodium fluid to thermally-bond the heat source (fuel) to the heat sink (heat pipe), thus relegating thermal stress to be an insignificant concern.

\subsection{Design Options}

It must be noted that there are other design options that can possibly be implemented in the LANL concept to mitigate the excessive calculated stress levels in the monolith and even perhaps establish an acceptable thermal margin. One option might be to increase the thickness of the stainless steel webs where the stresses are highest. This should help flatten thermal gradients and stiffen the web, but would also increase the reactor footprint. Also, the reactor power and operating temperature could be decreased and limited to a level below $675^{\circ} \mathrm{C}$. There could be pushback on this second option, since the prime goal of the micro-reactor is to produce electricity. In order to maximize electrical output, the reactor power and monolith temperature need to operate at levels as high as possible. Maximized and optimal operating conditions must also include a thermal margin for safety. Other design options may exist as well; these might include the use of a higher-temperature monolith material, but this would require substantial time and effort for development of acceptable ASME code case.

The high monolith thermal stress levels at normal operating conditions, excessive levels under offnormal conditions, and the currently unknown overlaid stress consequences of a seismic event led the INL 
PIRT team to core design alternatives that could replace the monolithic steel core structure. Two new non-monolithic core design alternatives are proposed here by INL (Designs A and B).

\subsection{Design A}

Design A replaces the monolithic core structure with a collection of individual fuel elements. The fuel element design is unique, with a central heat pipe surrounded by a $\mathrm{UO}_{2}$ fuel pellet with cladding on both radial sides of the pellet (inner and outer cladding). The heat pipe tube and cladding are made of stainless steel, and the total core mass of stainless steel is comparable to that of the LANL design. The steel is in the form of fuel cladding. Its intended use is solely to contain the $\mathrm{UO}_{2}$ fuel and fission gases; it is not meant to act as a core structural component. The Design A fuel elements are independent and unrestrained in the core (i.e. they are free to move axially against one another). This freedom of motion could potentially lower thermo-mechanical stress and eliminate those stresses induced by the flexure of the monolithic structure as a whole. The Design A fuel element also has azimuthal symmetry about the central steel heat pipe tube and the surrounding inner steel cladding. This symmetry simplifies the radial stress patterns and should also result in lower stress levels. The annular fuel pellet in a single Design A contains a $\mathrm{UO}_{2}$ fuel mass roughly equivalent to the six monolith fuel pellet stacks around a heat pipe. This annular pellet design is an efficient $\mathrm{UO}_{2}$ loading pattern, which consequently frees up precious active core space, allowing for both inner and outer fuel cladding. The outer radial surface of the fuel pellet is hexagonal, as is the outer clad tube. This hexagonal fuel element shape in turn permits an efficient packing of the fuel elements in the core. At the outer clad interface between fuel elements, the thermal gradients and stress levels are relatively small and inconsequential.

The Design A fuel element does, however, have four gas gaps, or four thermal resistances, between the four element components (heat pipe, inner clad, $\mathrm{UO}_{2}$ pellet, and outer clad). Gas gaps can potentially elevate core temperatures slightly. The LANL monolith core structure has only one gas gap, or thermal resistance, namely the gap between the $\mathrm{UO}_{2}$ fuel pellet and the monolith. This gap, however, will be filled with pressurized helium gas to boost the gas gap thermal conductance and minimize any radial temperature profile increase. The four gas gaps in the Design A elements are located in the element as follows: (1) between the heat pipe outer surface and the inner clad, (2) between the inner clad and the $\mathrm{UO}_{2}$ fuel pellet, (3) between the $\mathrm{UO}_{2}$ fuel pellet and the outer clad, and (4) between the outer clads of adjacent fuel elements. The first gap could potentially be eliminated by co-extrusion of the heat pipe and inner clad. The second and third gaps will be thermally bonded using pressurized helium gas (as in the LANL case) and by minimizing the gap widths by sizing the gaps to close by thermal expansion at operating temperatures. The fourth gap can either be designed to close at operational temperatures through thermal expansion, or possibly be thermally bonded with a liquid metal, if necessary. The first gap could also be liquid metal bonded, if co-extrusion is not used. Options exist to reduce the thermal resistances associated with the four gaps.

Perhaps the biggest selling point for Design A is not just the potential to significantly reduce thermal stresses in the in-core steel structures, but the ease of fabrication of all the fuel element components with existing manufacturing technologies. Only the hexagonal $\mathrm{UO}_{2}$ fuel pellet will require some fabrication testing to achieve the appropriate sintered density and dimensions. Fabrication of the fuel pellet is doable today with current fuel fabrication technologies available at INL for experimentation and testing. 


\subsection{Design B}

The active core of Design B is even simpler than Design A. The Design B core is composed of fuel pins and heat pipes in a liquid metal sodium bath. The high-conductivity sodium thermally bonds the fuel pins to the heat pipes. The configuration of the fuel pin and heat pipe arrays is identical to that of the LANL concept, except the pitch is slightly larger in order to accommodate thin spacer plates that separate and hold the fuel pins and heat pipes in place. The fuel pins are clad. Replacement of the parasitic steel monolith with non-parasitic liquid sodium provides a boost in core reactivity, which easily compensates for the reactivity loss due to the increase in array pitch. The use of liquid metal sodium also eliminates the in-core thermal stress issues associated with the LANL monolith.

Loss of the liquid metal sodium in Design B may seem at first to be a potential neutronic concern. After all, this is a fast reactor, and some fast reactor designs have had small positive void coefficients in some operating regimes. However, the compact SPR has a calculated negative void coefficient of reactivity-an excellent safety feature. Second, the loss of sodium is deemed to be a low probability accident scenario due to the double-wall containment structure and the vertical orientation of the reactor. The double walls could be fabricated as two individual seamless tanks composed with top plates welded to the tanks. Only a high-kinetic energy projectile could conceivably breach both tank sidewalls and drain the sodium from the reactor. A loss of the sodium in one core segment could potentially uncover the fuel pins and degrade the high-conductance thermal path between fuel pin and heat pipe, which could in turn allow the decay heat to heat the fuel pins to excessive temperatures. Fortunately, stainless steel has a relatively high melting point of $1,510^{\circ} \mathrm{C}$. Careful design could allow sufficient decay heat to exit the core through radial and axial conduction to the side, upper, and lower reflectors, plus radial conduction via the spacer plates to the heat pipes, thus reducing the impact of a loss-of-sodium event to a manageable condition. The six-segment core, each segment having a double tank containment, would also serve to limit the extent of a loss-of-sodium event.

Like Design A, fabrication of the Design B core components can all be done with existing technologies and vendors. The transportability of liquid sodium, however, poses potential regulatory challenges.

\subsection{Heat Pipes}

Heat pipes are efficient heat transport devices, and their use in nuclear reactors to transport (lift) fission heat out of the reactor core is a novel application. The heat pipe working fluid is specifically designed for particular operational temperature ranges, and for the SPR, which operates in the $650-750^{\circ} \mathrm{C}$ temperature range, potassium-filled heat pipes are most efficient and will provide the greatest operating margin. Heat pipes have no moving parts (pumps, valves, or loop pipes) and, therefore, naturally avoid the standard loss-of-coolant accident scenarios associated with all commercial power reactors today. When a heat pipe is first fabricated, it is sized for length and inner diameter based on its intended application and desired lift capacity, then loaded with a wick and a specific amount of potassium, airevacuated, sealed, and left in vacuum. At normal operating conditions, alkali heat pipes optimally operate at vapor pressures $\leq 0.1 \mathrm{MPa}(\leq 14.5 \mathrm{psi})$, which helps to maintain a subsonic condition [7]. Therefore, even at hot operating conditions, the heat pipes are at low pressures and do not present a concern relative to high-pressure explosions or structural ruptures.

Heat pipes have other beneficial properties as well. Heat pipes operate at temperatures below their standard boiling points, due to sub-atmospheric working fluid vapor pressures inside the heat pipes. 
Potassium boils under standard temperature and pressure conditions at around $1,032^{\circ} \mathrm{C}$, but in the SPR heat pipes, the vapor-liquid operating temperature is approximately $650-750^{\circ} \mathrm{C}$. During operation, the heat pipes are nearly isothermal along their entire length. Another remarkable feature of heat pipes is the ability to self-adjust their lift capacity due to variations in the reactor core power. Over-power transients, due to small inadvertent reactivity insertions, are easily accommodated. This self-adjustment or heat flux balancing via temperature changes also applies to failed heat pipes, where adjacent heat pipes can naturally pick up the added heat flux load from the failed pipe.

With no moving parts, these heat pipe devices can efficiently transport considerable amounts of heat from one section of pipe (heated) to the other (cooled) in a two-phase counter flow with vapor in the center of the pipe and liquid flowing on the sidewall and wick. In all three SPR concepts, the heat pipe will be 4.0 meters $(13.1 \mathrm{ft}$.) in length and have an inner diameter of $1.575-\mathrm{cm}$. In the LANL and Design B concepts, both concepts will use 1,224 heat pipes to lift $5 \mathrm{MWt}$ core fission heat, which translates into approximately $4.1 \mathrm{~kW} /$ pipe. Design A currently has 1,134 heat pipes, and each pipe will need to lift, on average, $4.4 \mathrm{~kW} /$ pipe, or slightly more than the LANL and Design B concepts. In addition, the large numbers of heat pipes in the SPR cores provides not only backup heat life capability, but also adds to system reliability due to the redundancy.

Heat pipes in a nuclear reactor core have one obvious downside, especially for compact, fastspectrum reactor cores like the SPR. Fast neutrons (for that matter, neutrons of all energies) can readily leak out of the core through the heat pipes. The heat pipes will contain only a small amount or mass of potassium ( 100 g), not enough to scatter and reflect neutrons back into the core. In fact, most of the inner volume of the heat pipe is low-density potassium vapor. With a large number of heat pipes extending out one of the axial faces of the active core, a significant cross sectional area of that core face is available for neutrons to stream directly out the core (neutron leakage). The three SPR concepts employ either 1,224 or 1,134 heat pipes, and it is estimated that $30 \%$ or $41 \%$ of the cross-sectional area, respectively, is essentially open for neutrons to escape. High neutron leakage translates directly into loss of core reactivity. Reactivity loss can be compensated by increasing the uranium enrichment, core fuel load (core size increase), or outer side reflector thickness, but perhaps the most obvious solution would be to simply reduce the inner diameter of all the heat pipes, thus reducing the streaming surface area and leakage. Unfortunately, reducing the heat pipe inner diameter also reduces the heat pipe lift capacity and operating margin. Therefore, a heat pipe inner diameter made as large as possible is always most desirable. The inner heat pipe diameter is thus an important variable to optimize.

LANL has provided a preliminary specification for a heat pipe design to be used in the SPR (Appendix E). This heat pipe design has been independently evaluated by INL and will operate in an acceptable temperature range, comfortably below the sonic and capillary limits, even in the event of two adjacent heat pipe failures. The heat pipe should easily operate in a steady-power mode for the 5-year lifetime of the reactor at $675^{\circ} \mathrm{C}$ and in the neutron-irradiation environment as well. Neutron fast fluence can induce material hardening, but fluence levels and material damage over 5 years are very minimal (1.9 dpa maximum) and should be inconsequential to in-core stainless steel.

Activation of the potassium working fluid will occur and over a 5-year irradiation period and will produce at least three radionuclides with reportable curie (Ci) activities longer than a few days (Table 1). The activities, however, are relatively small and in consequential. For a heat pipe breach, especially in the condenser section above the core, these radionuclides could contaminate the primary heat exchanger system and be expelled to the outside environment. 
Table 1. Activation products in a single SPR heat pipe with $100 \mathrm{~g}$ potassium loading.

\begin{tabular}{|c|c|c|c|}
\hline Radioisotope & Half-life & Decay Mode & Activity \\
\hline $\mathrm{Ar}-39$ & $269 \mathrm{yr}$ & beta- decay (no gamma) & $0.255 \mathrm{Ci}$ \\
\hline $\mathrm{K}-42$ & $12.36 \mathrm{hr}$ & beta-/gamma decay & $0.213 \mathrm{Ci}$ \\
\hline $\mathrm{Cl}-36$ & $301,000 \mathrm{yr}$ & beta+/beta- emitter & $54.6 \mu \mathrm{Ci}$ \\
\hline
\end{tabular}

A properly fabricated heat pipe should operate at its rated performance levels, but heat pipe performance may degrade over time due to steel material property changes. Examples include grain growth and elemental migration in the steel due to high temperature operation or corrosion of the steel heat pipe wall and wick due to chemical reactions with impurities in the steel or working fluid [7]. Other, more mundane problems associated with heat pipe performance, such as damaged wicks, improper loading of the wick or potassium charge, and improper sealing of the heat pipe can all be handled with a rigorous quality assurance program as part of the fabrication process line.

\subsection{Common Characteristics}

INL's new active core designs have intentionally been designed with operating and performance characteristics and metrics comparable to the LANL concept. The three design concepts (LANL, Design A, Design B) are therefore very similar in most respects despite having different active core design geometries. Some of the common characteristics include: core power, core size, use of heat pipes, $\mathrm{UO}_{2}$ fuel, in-core steel, high temperature, excess reactivity, neutron spectrum, burnup, and core lifetime. In addition, ex-core features and components will also remain virtually the same; some of these include the heat pipe design, power conversion unit, alumina side reflector, and number of control drums, among others. There will, however, be some inevitable design differences, including dimensions, geometry, and number of heat pipes and fuel elements, plus some deliberate differences, such as reactor orientation.

Table 2 provides a side-by-side comparison of the key reactor parameters to highlight the similarities and differences.

Table 2. Nominal reactor design parameters for Design A, Design B, and the LANL design concepts.

\begin{tabular}{|l|l|l|l|}
\hline \multicolumn{1}{|c|}{ REACTOR } & \multicolumn{1}{c|}{ Design A } & \multicolumn{1}{c|}{ Design B } & \multicolumn{1}{c|}{ LANL } \\
\hline Reactor thermal power & $5 \mathrm{MW}$ & $5 \mathrm{MW}$ & $5 \mathrm{MW}$ \\
\hline Reactor electrical output & $2 \mathrm{MWe}$ & $2 \mathrm{MWe}$ & $2 \mathrm{MWe}$ \\
\hline Reactor core orientation & Vertical & Vertical & Horizontal \\
\hline Cycle length & 5 years & 5 years & 5 years \\
\hline Coolant system & Heat pipes & Heat pipes & Heat pipes \\
\hline Reactor structure & Type 316 Stainless steel & Type 316 Stainless steel & Type 316 Stainless steel \\
\hline $\begin{array}{c}\text { POWER CONVERSION } \\
\text { SYSTEM }\end{array}$ & & & \\
\hline Conversion cycle & Open-air Brayton & Open-air Brayton & Open-air Brayton \\
\hline Primary heat exchanger & Air convection over HPs & Air convection over HPs & Air convection over HPs \\
\hline
\end{tabular}




\begin{tabular}{|c|c|c|c|}
\hline Maximum air temperature & $675^{\circ} \mathrm{C}$ & $675^{\circ} \mathrm{C}$ & $675^{\circ} \mathrm{C}$ \\
\hline Cycle efficiency & $40.3 \%$ & $40.3 \%$ & $40.3 \%$ \\
\hline \multicolumn{4}{|l|}{ FUEL } \\
\hline Fuel form & $\mathrm{UO}_{2}$ & $\mathrm{UO}_{2}$ & $\mathrm{UO}_{2}$ \\
\hline Theoretical density (TD) & $10.96 \mathrm{~g} / \mathrm{cm}^{3}$ & $10.96 \mathrm{~g} / \mathrm{cm}^{3}$ & $10.96 \mathrm{~g} / \mathrm{cm}^{3}$ \\
\hline Percent of TD & $96.0 \%$ & $96.0 \%$ & $96.0 \%$ \\
\hline Density & $10.52 \mathrm{~g} / \mathrm{cm}^{3}$ & $10.52 \mathrm{~g} / \mathrm{cm}^{3}$ & $10.52 \mathrm{~g} / \mathrm{cm}^{3}$ \\
\hline U-235 enrichment & $19.75 \mathrm{wt} \%$ & $19.75 \mathrm{wt} \%$ & $19.75 \mathrm{wt} \%$ \\
\hline Fuel pellet form & Solid pellet & Solid pellet & Solid pellet \\
\hline Fuel pellet geometry & $\begin{array}{l}\text { Hexagonal with central } \\
\text { hole }\end{array}$ & Cylindrical & Cylindrical \\
\hline Fuel pellet thicknesses & $\begin{array}{l}0.340 \mathrm{~cm}(\min ) \\
0.538 \mathrm{~cm}(\max )\end{array}$ & $1.492 \mathrm{~cm} \mathrm{dia}$. & $1.412 \mathrm{~cm}$ dia. \\
\hline Central pellet hole diameter & $1.8806 \mathrm{~cm}$ & Solid cylindrical & Solid cylindrical \\
\hline \multicolumn{4}{|l|}{ HEAT PIPES } \\
\hline Number of HPs in-core & 1,134 & 1,224 & 1,224 \\
\hline Average HP power & $4.41 \mathrm{~kW}$ & $4.08 \mathrm{~kW}$ & $4.08 \mathrm{~kW}$ \\
\hline Pipe wall material & SS316 & SS316 & SS316 \\
\hline Pipe inner diameter & $1.575 \mathrm{~cm}$ & $1.575 \mathrm{~cm}$ & $1.575 \mathrm{~cm}$ \\
\hline Pipe outer diameter & $1.757 \mathrm{~cm}$ & $1.757 \mathrm{~cm}$ & ---- \\
\hline Pipe wall thickness & $1.0 \mathrm{~mm}$ & $1.0 \mathrm{~mm}$ & $1.0 \mathrm{~mm}$ (min.) \\
\hline HP-to-HP pitch & $2.78 \mathrm{~cm}$ & $1.80 \mathrm{~cm}$ & $1.60 \mathrm{~cm}$ \\
\hline Working fluid & Potassium (vapor/liquid) & Potassium (vapor/liquid) & Potassium (vapor/liquid) \\
\hline Potassium mass & 100 grams/pipe & 100 grams/pipe & 100 grams/pipe \\
\hline Potassium temperature & $675^{\circ} \mathrm{C}$ & $675^{\circ} \mathrm{C}$ & $675^{\circ} \mathrm{C}$ \\
\hline HP length (evaporator) & $1.5 \mathrm{~m}$ & $1.5 \mathrm{~m}$ & $1.5 \mathrm{~m}$ \\
\hline HP length (adiabatic) & $0.4 \mathrm{~m}$ & $0.4 \mathrm{~m}$ & $0.4 \mathrm{~m}$ \\
\hline HP length (condenser) & $2.1 \mathrm{~m}$ & $2.1 \mathrm{~m}$ & $2.1 \mathrm{~m}$ \\
\hline HP total length & $4.0 \mathrm{~m}$ & $4.0 \mathrm{~m}$ & $4.0 \mathrm{~m}$ \\
\hline \multicolumn{4}{|l|}{ FUEL ELEMENTS } \\
\hline No. of fuel elements in-core & 1,134 & 2,112 & 2,112 \\
\hline Element geometry & hexagonal & cylindrical & cylindrical \\
\hline Geometry & $\begin{array}{l}\text { Central heat pipe } \\
\text { surrounded by clad } \mathrm{UO}_{2} \\
\text { fuel }\end{array}$ & $\begin{array}{l}\text { Fuel pins }+ \text { heat pipes } \\
\text { (hexagonal arrays) }\end{array}$ & $\begin{array}{l}\text { Fuel pellet and heat pipe } \\
\text { channels in monolith } \\
\text { (hexagonal arrays) }\end{array}$ \\
\hline No. of gas gaps & 4 & 1 & 1 \\
\hline Thermal bonding media & Helium/liquid metal & Helium/liquid metal & Helium \\
\hline Helium gas pressure (fuel) & $45 \mathrm{psi}$ & $45 \mathrm{psi}$ & $45 \mathrm{psi}$ \\
\hline Gap thicknesses & $0.0065 \mathrm{~cm}$ & $0.0065 \mathrm{~cm}$ & $0.0065 \mathrm{~cm}$ \\
\hline Fuel pellet stack length & $150.0 \mathrm{~cm}$ & $150.0 \mathrm{~cm}$ & $150.0 \mathrm{~cm}$ \\
\hline Fuel clad material & SS316 & SS316 & SS316 \\
\hline Clad inner diameter & $\begin{array}{l}0.8939 \mathrm{~cm} \text { (inner clad) } \\
1.2867 \mathrm{~cm} \text { (outer clad) }\end{array}$ & $1.505 \mathrm{~cm}$ & $\begin{array}{ll}--- \\
\end{array}$ \\
\hline Clad outer diameter & $\begin{array}{l}0.9339 \mathrm{~cm} \text { (inner clad) } \\
1.3867 \mathrm{~cm} \text { (outer clad) }\end{array}$ & $1.565 \mathrm{~cm}$ & $\begin{array}{ll}--- \\
\end{array}$ \\
\hline
\end{tabular}




\begin{tabular}{|c|c|c|c|}
\hline $\begin{array}{l}\text { Clad thickness or minimum } \\
\text { web thickness }\end{array}$ & $\begin{array}{l}0.4 \mathrm{~mm} \text { (inner) } \\
1.0 \mathrm{~mm} \text { (outer) }\end{array}$ & $0.3 \mathrm{~mm}$ & $\begin{array}{l}1.00 \mathrm{~mm} \text { HP-to-fuel } \\
1.75 \mathrm{~mm} \text { fuel-to-fuel }\end{array}$ \\
\hline Cladding geometry & $\begin{array}{l}\text { Cylindrical tube (inner) } \\
\text { Hexagonal tube (outer) }\end{array}$ & Cylindrical tube & Monolith \\
\hline Fuel pin or element pitch & $2.78 \mathrm{~cm}$ & $1.8 \mathrm{~cm}$ & $1.6 \mathrm{~cm}$ \\
\hline Fission gas plenum & $2.0 \mathrm{~cm}$ & $2.0 \mathrm{~cm}$ & $20.0 \mathrm{~cm}$ \\
\hline \multicolumn{4}{|l|}{ CORE } \\
\hline Type & Hexagonal fuel elements & Fuel pins/heat pipes & Monolith \\
\hline Geometry & Hexagonal & Hexagonal (6 sectors) & Hexagonal (6 sectors) \\
\hline Core diameter & $101.2 \mathrm{~cm}$ flat-to-flat & $113.6 \mathrm{~cm}$ flat-to-flat & $101.2 \mathrm{~cm}$ flat-to-flat \\
\hline Active height & $150.0 \mathrm{~cm}$ & $150.0 \mathrm{~cm}$ & $150.0 \mathrm{~cm}$ \\
\hline Mass of $\mathrm{UO}_{2}$ in-core & $5.19 \mathrm{MT}$ & $5.83 \mathrm{MT}$ & $5.22 \mathrm{MT}$ \\
\hline Mass of U in-core & 4.57 MTU & $5.13 \mathrm{MTU}$ & 4.60 MTU \\
\hline Mass of ${ }^{235} \mathrm{U}$ & $904 \mathrm{~kg}$ & $1,015 \mathrm{~kg}$ & $908 \mathrm{~kg}$ \\
\hline BOL core k-effective & 1.02825 & 1.02417 & 1.02153 \\
\hline BOL excess reactivity & $\$ 3.82$ & $\$ 3.28$ & $\$ 2.93$ \\
\hline Burnup & $1.998 \mathrm{GWD} / \mathrm{MTU}$ & $1.566 \mathrm{GWD} / \mathrm{MTU}$ & 1.985 GWD/MTU \\
\hline Heavy metal burnup & $0.20 \%$ & $0.16 \%$ & $0.20 \%$ \\
\hline Mass of SS316 in-core & $2.03 \mathrm{MT}$ & $1.95 \mathrm{MT}$ & $2.57 \mathrm{MT}$ \\
\hline Mass of $\mathrm{Al}_{2} \mathrm{O}_{3}$ side reflector & $7.93 \mathrm{MT}$ & $8.59 \mathrm{MT}$ & $7.93 \mathrm{MT}$ \\
\hline BOL excess reactivity & $\$ 3.82$ & $\$ 3.28$ & $\$ 2.93$ \\
\hline \multicolumn{4}{|l|}{$\begin{array}{l}\text { NEUTRON } \\
\text { REFLECTORS }\end{array}$} \\
\hline $\begin{array}{l}\text { Number of neutron } \\
\text { reflectors }\end{array}$ & 3 (top, bottom, side) & 3 (top, bottom, side) & 3 (top, bottom, side) \\
\hline Side reflector outer radius & $77.85 \mathrm{~cm}$ & $84.05 \mathrm{~cm}$ & $77.85 \mathrm{~cm}$ \\
\hline Side reflector thickness & $19.4-27.3 \mathrm{~cm}$ & $18.5-27.3 \mathrm{~cm}$ & $19.4-27.3 \mathrm{~cm}$ \\
\hline Side reflector length & $200 \mathrm{~cm}$ & $200 \mathrm{~cm}$ & $200 \mathrm{~cm}$ \\
\hline Side reflector material & Alumina $\left(\mathrm{Al}_{2} \mathrm{O}_{3}\right)$ & Alumina $\left(\mathrm{Al}_{2} \mathrm{O}_{3}\right)$ & Alumina $\left(\mathrm{Al}_{2} \mathrm{O}_{3}\right)$ \\
\hline Alumina density & $3.9 \mathrm{~g} / \mathrm{cm}^{3}$ & $3.9 \mathrm{~g} / \mathrm{cm}^{3}$ & $3.9 \mathrm{~g} / \mathrm{cm}^{3}$ \\
\hline Top axial reflector thickness & $15.0 \mathrm{~cm}$ & $15.0 \mathrm{~cm}$ & $15.0 \mathrm{~cm}$ \\
\hline $\begin{array}{l}\text { Bottom axial reflector } \\
\text { thickness }\end{array}$ & $15.0 \mathrm{~cm}$ & $15.0 \mathrm{~cm}$ & $15.0 \mathrm{~cm}$ \\
\hline $\begin{array}{l}\text { Top/bottom reflector } \\
\text { material }\end{array}$ & $\begin{array}{l}\mathrm{SS} 316+\mathrm{BeO} \text { (above } \\
\text { fuel) }\end{array}$ & $\begin{array}{l}\mathrm{SS} 316+\mathrm{BeO} \text { (above } \\
\text { fuel) }\end{array}$ & $\begin{array}{l}\mathrm{SS} 316+\mathrm{BeO} \text { (above } \\
\text { fuel) }\end{array}$ \\
\hline $\begin{array}{l}\text { Beryllium oxide }(\mathrm{BeO}) \\
\text { density }\end{array}$ & $3.01 \mathrm{~g} / \mathrm{cm}^{3}$ & $3.01 \mathrm{~g} / \mathrm{cm}^{3}$ & $3.01 \mathrm{~g} / \mathrm{cm}^{3}$ \\
\hline \multicolumn{4}{|l|}{ CONTROL DRUMS } \\
\hline Number of control drums & 12 & 12 & 12 \\
\hline Location & Side reflector & Side reflector & Side reflector \\
\hline Drum outer diameter & $25.0 \mathrm{~cm}$ & $25.0 \mathrm{~cm}$ & $25.0 \mathrm{~cm}$ \\
\hline Drum axial length & $200 \mathrm{~cm}$ & $200 \mathrm{~cm}$ & $200 \mathrm{~cm}$ \\
\hline Drum control banks & 6 & 6 & 6 \\
\hline Control material & $\mathrm{B}_{4} \mathrm{C}$ & $\mathrm{B}_{4} \mathrm{C}$ & $\mathrm{B}_{4} \mathrm{C}$ \\
\hline
\end{tabular}




\begin{tabular}{|l|l|l|l|}
\hline Boron-10 enrichment & $90 \%$ & $90 \%$ & $90 \%$ \\
\hline Boron carbide density & $2.51 \mathrm{~g} / \mathrm{cm}^{3}$ & $2.51 \mathrm{~g} / \mathrm{cm}^{3}$ & $2.51 \mathrm{~g} / \mathrm{cm}^{3}$ \\
\hline $\begin{array}{l}\text { Control material } \\
\text { configuration }\end{array}$ & $\begin{array}{l}\text { Crescent-shape of } \mathrm{B}_{4} \mathrm{C} \\
\text { (edge of drum) }\end{array}$ & $\begin{array}{l}\text { Crescent-shape of } \mathrm{B}_{4} \mathrm{C} \\
\text { (edge of drum) }\end{array}$ & $\begin{array}{l}\text { Crescent-shape of } \mathrm{B}_{4} \mathrm{C} \\
\text { (edge of drum) }\end{array}$ \\
\hline Single CD worth & $\$ 1.15$ & $\$ 1.08$ & $\$ 1.20$ \\
\hline Total worth of all CDs & $\$ 13.83$ & $\$ 12.97$ & $\$ 14.42$ \\
\hline $\begin{array}{l}\text { EMERGENCY } \\
\text { CONTROL RODS }\end{array}$ & & & \\
\hline $\begin{array}{l}\text { Number of emergency } \\
\text { control rods }\end{array}$ & 2 & 2 & 2 \\
\hline $\begin{array}{l}\text { Location in-core } \\
\text { Geometry }\end{array}$ & $\begin{array}{l}\text { Inside core central } \\
\text { hexagon volume }\end{array}$ & $\begin{array}{l}\text { Inside core central } \\
\text { hexagon volume }\end{array}$ & $\begin{array}{l}\text { Inside core central } \\
\text { hexagon volume }\end{array}$ \\
\hline Control material & 1 solid rod & 1 solid rod \\
annular tube & $\mathrm{B}$ C & $\mathrm{B} 4 \mathrm{C}$ tube & $\begin{array}{l}1 \mathrm{solid} \mathrm{rod} \\
1 \text { annular tube }\end{array}$ \\
\hline Boron-10 enrichment & $90 \%$ & $90 \%$ & $\mathrm{~B} 4 \mathrm{C}$ \\
\hline Boron carbide density & $2.51 \mathrm{~g} / \mathrm{cm}^{3}$ & $2.51 \mathrm{~g} / \mathrm{cm}^{3}$ & $90 \%$ \\
\hline Solid rod outer radius & $5.6 \mathrm{~cm}$ & $5.6 \mathrm{~cm}$ & $2.51 \mathrm{~g} / \mathrm{cm}^{3}$ \\
\hline Annular tube inner radius & $6.85 \mathrm{~cm}$ & $6.85 \mathrm{~cm}$ & $5.6 \mathrm{~cm}$ \\
\hline Annular tube outer radius & $8.85 \mathrm{~cm}$ & $8.85 \mathrm{~cm}$ & $6.85 \mathrm{~cm}$ \\
\hline Length & $200 \mathrm{~cm}$ & $200 \mathrm{~cm}$ & $8.85 \mathrm{~cm}$ \\
\hline
\end{tabular}

The goal in developing Designs A and B was not to replace the LANL concept, but rather to offer an alternative active core design that does not use a stainless steel monolith structure. The alternative design concepts are simple and should be much easier to manufacture while retaining the majority of the LANL concept components, features, and performance.

\subsection{Neutron Spectra}

The three concepts also exhibit similar fast spectra and burnup characteristics. Figure 2 shows the calculated in-core neutron spectra for the three active core concepts. Despite some variation in the $\mathrm{UO}_{2}$, steel, potassium, and sodium mass loadings between the cores, the three spectra are virtually identical, all hard, fast, fission-like spectra. Some variation in the spectra exist in the low energy range, but the flux magnitude here is very small, as to be inconsequential neutronically. More importantly, the similarity in spectra lead to very comparable burnups at $5 \mathrm{MWt}$ power. 


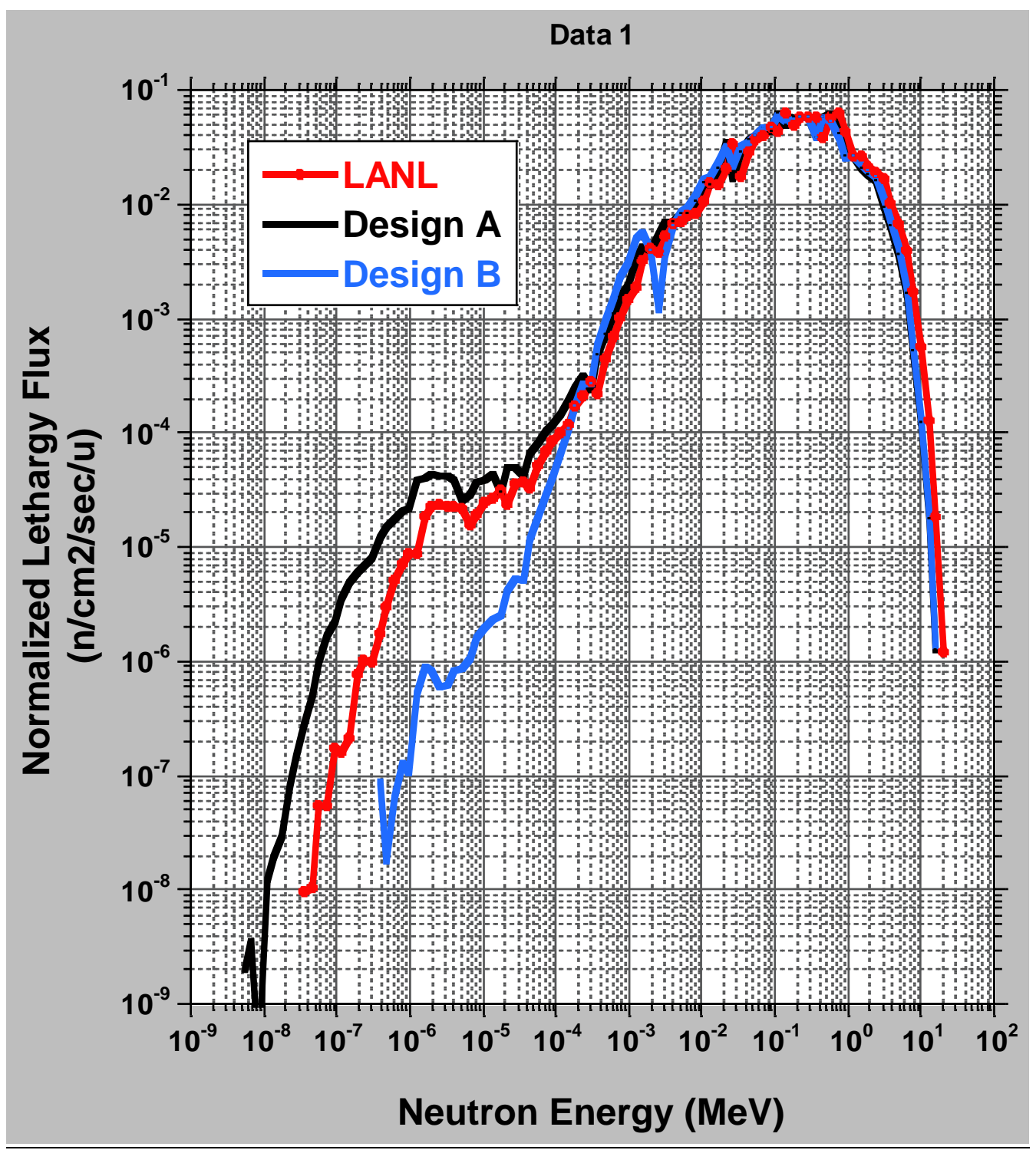

Figure 2. Neutron spectra comparison for the three reactor design concepts.

\subsection{Burnup}

Figure 3 shows calculated reactivity letdown curves for the three designs. The curves are flat and behave similarly, as expected, due to the similar neutron spectra and the same assumed total core power (5 MWt). The curves are, however, shifted vertically from one another due to the differences in the initial beginning-of-life excess reactivities. Extrapolation of all three curves through time shows the potential for very long-lived core lifetimes. In fact, Design A would not go sub-critical even after 50 years of operation at $5 \mathrm{MWt}$. The long-life potential is due to the inherent core physics. The conversion ratio, or ratio of fissile material produced divided by the fissile material destroyed, is $>0.99$ over the 5 -year cycle length. These three reactor cores will produce fissile atoms at virtually the same rate as they are consumed. The buildup of negative reactivity fission products over time and the very slow consumption of fissile fuel ultimately leads to sub-criticality, but only after many decades. 
The initial core loading of $\mathrm{UO}_{2}$ in all three core design concepts is $>5.2 \mathrm{MT}$ with the uranium heavy metal loadings all >4.5 MTU. These are fairly substantial core mass loadings. The $\mathrm{UO}_{2}$ mass is equivalent to approximately eleven PWR 17x17 fuel assemblies. Consumption of the initial core heavy metal at $5 \mathrm{MWt}$ over 5 years is only $0.2 \%$, or about 2 GWD/MTU. These heavy metal burnups are very small, which contributes to the long-life behavior of the cores, but also reflects poor uranium utilization.

The flatness of the letdown curves implies not only a good conversion ratio for the LEU fuel, but also the need for only a small amount of initial or beginning-of-life core excess reactivity. From Table 1, the excess reactivity for Design A, Design B, and LANL is calculated to be only a few dollars, or $\$ 3.82$, $\$ 3.28$, and $\$ 2.93$, respectively. Although the required initial excess reactivity can be low, these cores are very sensitive to small dimensional changes in the core (e.g. web thickness, pitch, pellet diameter, steel mass, and clad thickness). Including a reactivity margin would be prudent, especially for as-built, first-ofa-kind cores like Design B and the LANL concept. Both could potentially undershoot the goal excess core reactivity, leading to a limited core life or even a subcritical reactor, thereby rendering the prototype core useless, since additional fuel cannot be added to these two cores once built. Design A, however, has the ability to add additional fuel elements (reactivity) to the core periphery, an advantage for Design A relative to the other two concepts.

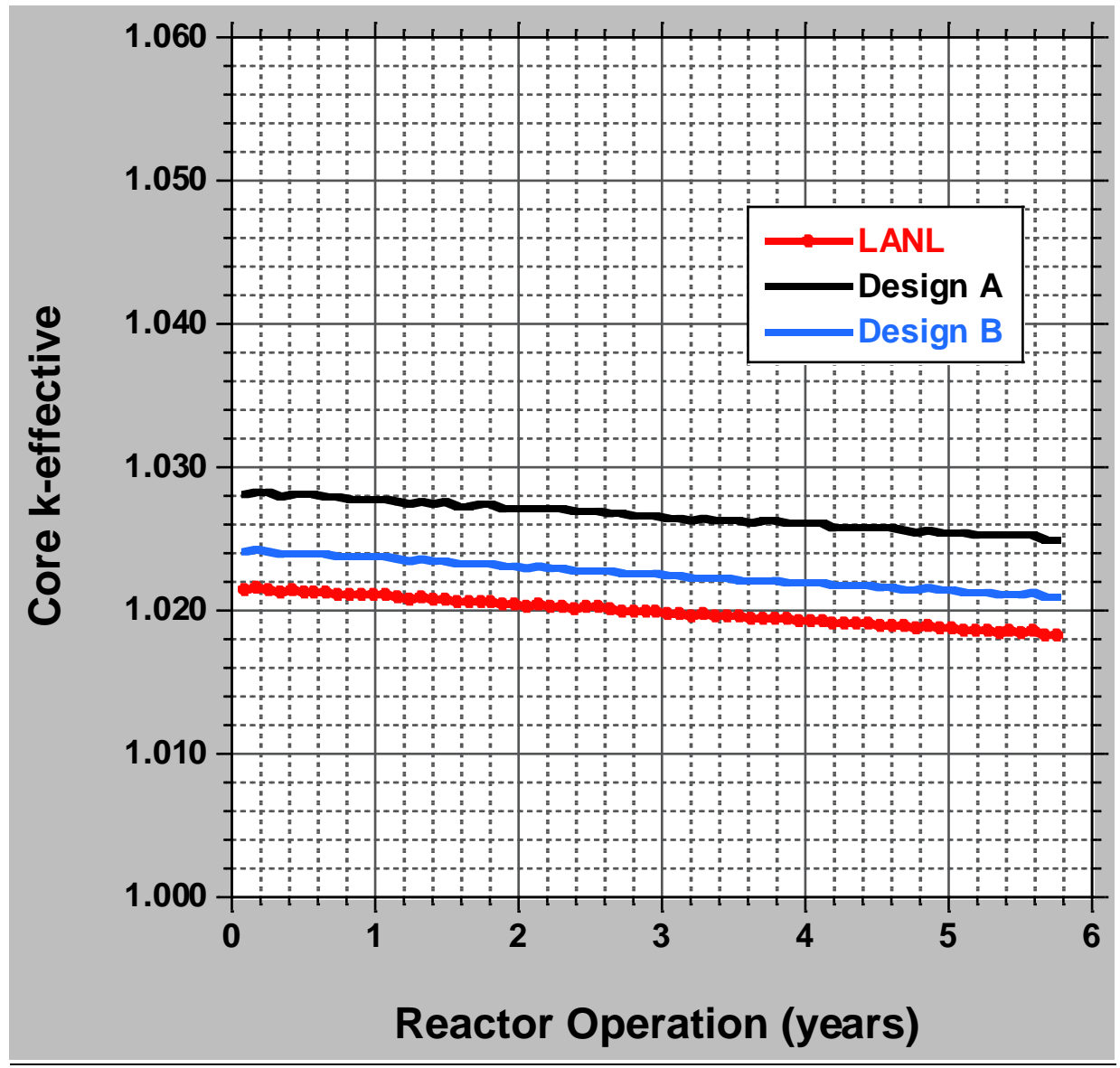

Figure 3. Reactivity letdown curves for the three reactor design concepts. 


\subsection{Radiation Streaming}

Another common characteristic between the three core designs is radiation streaming out the heat pipes. Both neutrons and gamma rays can leak out of the core through the heat pipes. The 4-meter-long heat pipes contain only a small amount of potassium $(\sim 100 \mathrm{~g})$, mostly potassium vapor by volume. The small potassium mass is not substantial enough to scatter and reflect significant quantities of neutrons back into the core, which causes significant neutron leakage and core reactivity loss. The potassium mass is also insufficient to attenuate gamma radiation emanating from the core; in addition, potassium is a relatively low-Z material $(\mathrm{Z}=19)$. For the LANL and Design B concepts, the end of the reactor core in which the heat pipes penetrate the reflector, $30 \%$ of the reflector surface is essentially unobstructed for radiation to spew out of the core. In fact, the fast neutron flux at 1 meter and 2 meters above the core in the heat pipe condenser forest is estimated to be $3.4 \mathrm{E}+11$ and $1.1 \mathrm{E}+11 \mathrm{n} / \mathrm{cm}^{2} / \mathrm{sec}$, respectively. At core midplane, where the fast flux is most intensive, the flux magnitude is $5.5 \mathrm{E}+13 \mathrm{n} / \mathrm{cm}^{2} / \mathrm{sec}$. In the condenser forest, the flux decreases by only a little more than two orders of magnitude. These are significant ex-core neutron fluxes which can activate the heat pipes and heat exchangers and pose a radiation hazard to personnel. The corresponding neutron dose rates at the 1-meter and 2-meter elevations above the core are approximately $20 \mathrm{MRem} / \mathrm{hr}$ and $6 \mathrm{MRem} / \mathrm{hr}$, respectively; gamma-ray dose rates are lower, but still significant at $200 \mathrm{kRad} / \mathrm{hr}$ and $40 \mathrm{kRad} / \mathrm{hr}$, respectively. A biological shield (e.g., concrete bunker) will need to fully encase the reactor system in order to prevent excessive radiation exposures to reactor personnel.

\subsection{Power Conversion Unit}

A power conversion unit will take heated air blown over the heat pipes $\left(675^{\circ} \mathrm{C}\right.$ condenser sections extended above the reactor core) and drive a small commercial gas turbine to generate electricity. For the SPR operating at $5 \mathrm{MWt}$, and using a heat-recuperated air Brayton cycle, a thermal efficiency of up to $40.3 \%$ could be obtained for an optimal compressor pressure ratio of 2.48 . This thermal efficiency would correspond to an optimal electrical power output of approximately $2.02 \mathrm{MWe}$. For more detail on the power conversion unit, including computer codes, computer models, parametric studies, and analysis results, see reference [6], Appendix G. 


\section{LANL CONCEPT}

This section gives a description of the LANL concept along with previously identified strengths and weaknesses excerpted from reference [6].

\subsection{Description}

The Special Purpose Reactor, or the Mega-Power nuclear reactor, is a LANL micro-reactor design concept [3][4][5]. The basic system is substantially different from other current power reactor systems. Basic characteristics include:

- Compact fast reactor

- Low power: $5 \mathrm{MWt}(2 \mathrm{MWe})$

- Heat pipe cooling (no water)

- Low-enriched $\mathrm{UO}_{2}$ fuel (19.75\% enriched)

- Stainless steel monolithic core to contain $\mathrm{UO}_{2}$ pellets and heat pipes

- Self-regulating in-core physics aids active control system

- No moving parts, valves, pumps, or high-pressure systems

- Passive decay heat removal

- Open-air recuperated air Brayton power conversion unit.

The nominal core thermal power is $5 \mathrm{MWt}$ and using the Brayton thermodynamic cycle can produce approximately 2 MWe. The core lifetime is specified to be 5 years. It consists of a hexagonal, Type 316 stainless steel (SS316) monolithic structure containing 5.22 MT of uranium-oxide $\left(\mathrm{UO}_{2}\right)$ fuel pins and 1,224 liquid metal potassium $(\mathrm{K})$ heat pipes operating at $675^{\circ} \mathrm{C}$. Figure 4 shows some of the major reactor structures.

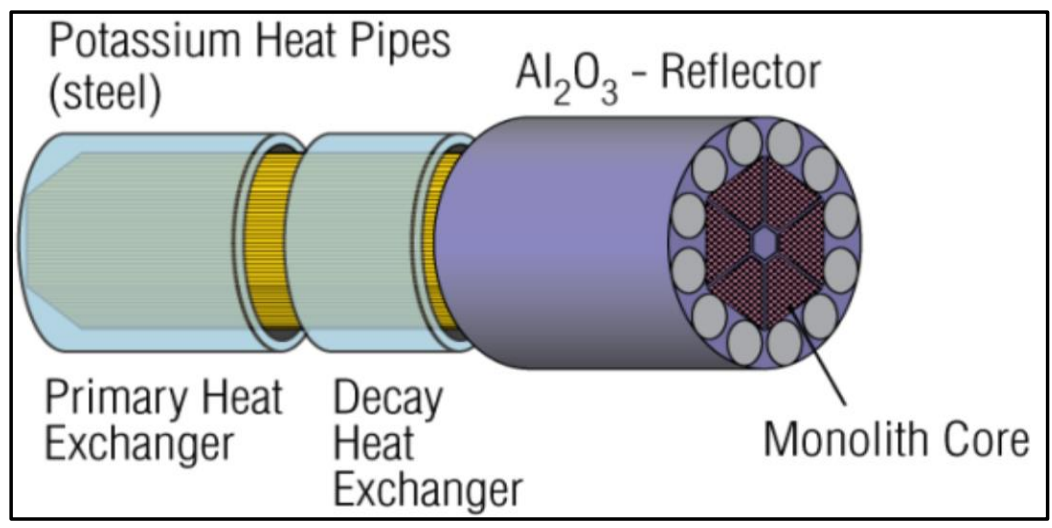

Figure 4. Special Purpose Reactor concept schematic.

The heat pipes remove the heat from the monolith as the potassium liquid in the heat pipes is vaporized in the evaporator section and transported as vapor to the condenser section. The hot vapor subsequently deposits the latent heat of evaporation in the condenser section of the heat pipe. The condenser region can be sized to accommodate multiple heat exchangers, such as one primary heat exchanger for power conversion and one or two additional heat exchangers for redundant decay heat 
removal. The reactor uses an alumina $\left(\mathrm{Al}_{2} \mathrm{O}_{3}\right)$ neutron side reflector, with 12 embedded control drums that contain an arc of boron-carbide $\left(\mathrm{B}_{4} \mathrm{C}\right)$ poison for reactivity control. The active part of the core is about 1 meter flat-to-flat and 1.5 meters high. The outer diameter of the $\mathrm{Al}_{2} \mathrm{O}_{3}$ reflector is 1.5 meters. In the proposed concept the monolith core is fabricated in six identical segments, forming a central hexagonal volume for two emergency shutdown control rods.

The SPR design is an innovative LANL design with many attractive safety features based on design simplicity. The unique core design is built around a solid steel monolith with channels for both heat pipes and fuel pellets. The monolith is stainless steel, and the fuel is commercial uranium oxide $\left(\mathrm{UO}_{2}\right)$, both well-characterized nuclear materials with high technology readiness levels. The use of heat pipes in nuclear reactors is new and perhaps not as familiar to the nuclear industry, but liquid metal heat pipe technology is mature and robust with a large experimental test database to support implementation of the technology into nuclear applications. The marriage of these three components makes the SPR concept unique and simple.

Use of the heat pipes in a reactor system addresses some of the most difficult reactor safety issues and reliability concerns present in current Generation II and III commercial nuclear reactors - in particular, loss of primary coolant. Heat pipes operate in a passive mode at very low pressure, less than an atmosphere. Each individual heat pipe contains only a small amount of working fluid (100 g), which is fully encapsulated in a sealed steel pipe. There is no primary cooling loop, hence none of the mechanical pumps, valves, or large-diameter primary loop piping typically found in all commercial reactors today. Heat pipes simply transport heat from the in-core evaporator section to the ex-core condenser in continuous isothermal vapor/liquid internal counter-flow. Heat pipes offer a new and unique means to remove heat from a reactor core.

Type 316 stainless steel and liquid metal potassium are compatible. Corrosion is not a significant issue. The uniform temperature distribution throughout the core and the small temperature drop from the fuel pin to heat pipe is intended to provide robustness in the ability to remove heat from the core in case of some heat pipe failures. The high thermal conductivity of the steel monolith will conduct the heat efficiently to the heat pipes, but the calculated thermal stresses and temperatures of the steel-in particular, the thin webbings between fuel and heat pipe channels - are of concern. A significant thermal design margin is inherent in the high temperature $\mathrm{UO}_{2}$ fuel.

Each fuel pin in the core is adjacent to three heat pipes for efficiency and redundancy. Overall there is a 1-to-2 heat pipe-to-fuel ratio throughout the core. The heat pipes have also been designed to operate well below the peak heat flux capability of the heat pipe, thereby allowing for a significant margin in the heat pipes in case of heat pipe failure or power transients within the core. The large number of in-core heat pipes is intended to increase system reliability and safety. Decay heat can also be removed by the heat pipes with the decay heat exchanger. The total potassium mass in all the heat pipes is estimated to be approximately $123 \mathrm{~kg}$. The presence of this mass in the core has virtually no impact on the core reactivity. The small radiative capture cross-sections of the potassium isotopes result in a negligible void coefficient. The reactivity insertion due to the total loss of all the potassium in the heat pipes is very small and inconsequential. 
The LANL reactor is a fast spectrum reactor. The core contains no moderating material, just steel, $\mathrm{UO}_{2}$, and a small amount of potassium liquid/vapor. The temperature coefficient of reactivity is strongly negative with negative feedback contributions from $\mathrm{UO}_{2}$ Doppler broadening, $\mathrm{UO}_{2}$ axial elongation due to thermal expansion, and thermal expansion of the steel monolith. Any transient power excursions would be mitigated quickly by the negative temperature feedback. The strong negative reactivity feedback $\left(-0.2 \phi /{ }^{\circ} \mathrm{C}\right)$, the small beginning-of-life excess core reactivity $(\$ 2.88)$, the use of control drums, and the high U-235 beta-effective (0.0073) will allow for easy control of the reactor power under both normal and accident conditions.

The primary purpose of the SPR system is to generate electricity. The LANL design uses a primary heat exchanger in the form of annular tubes around the ex-core condenser section of the heat pipes with inlet and outlet plenums at the condenser section ends. Implementation of such a heat exchanger design appears to be a formidable engineering challenge, given the dense packing of heat pipes. INL has instead assumed an open-air Brayton cycle with a shell-and-tube heat exchanger concept. INL has also designed a thermodynamic power cycle incorporating a recuperator into a standard Brayton cycle power conversion system. This system has been optimized for air pressure, flow, and temperature for each component in the power conversion system, resulting in an electrical output of greater than $2 \mathrm{MWe}$.

\subsection{PIRT-Identified Concerns}

Despite the many positive attributes of the SPR design, the INL has identified several design and manufacturing concerns using the PIRT technique. These concerns are documented in more detail in reference [6] and excerpted here for continuity.

The major design concerns identified included the following:

- Defense in Depth - Adequate defense in depth to the environment is essential. The monolith block and the heat pipe appear to be the only barriers between the fuel and the outside environment. If a tear or fracture develops in the monolith webbing, there is potential for a release of fission products from a failed heat pipe. The design should incorporate other defense-in-depth layers to eliminate direct pathways between the fuel and the environment. The design satisfies the single failure criterion, but that is not defense in depth. The likelihood of a heat pipe failure is high over the lifetime of the reactor (a similar situation to a steam generator tube leak in a PWR, which is also very likely over the life of the plant) and therefore, should not be regarded as adequate defense in depth.

- $\quad$ Monolith thermal stress - Under steady-state, normal operating conditions, the maximum calculated thermal stresses $\left(37.1 \mathrm{MPa}\right.$ at $\left.696^{\circ} \mathrm{C}\right)$ in the thin $1.75 \mathrm{~mm}$ steel monolith webbing between some fuel pin channels exceed the maximum $29 \mathrm{MPa}$ ASME pressure vessel code allowable limits at $700^{\circ} \mathrm{C}$. Web failure may be problematic.

- $\quad$ Single heat pipe failure - Failure of a single heat pipe results in localized steel monolith temperature and thermal stresses that far exceed the maximum allowable ASME pressure vessel code limits. The maximum calculated steel monolith thermal stress rises to $154.6 \mathrm{MPa}$ at $769^{\circ} \mathrm{C}$. These stresses occur in the steel webbing circumferentially around the heat pipes. Web failure may be problematic.

- Machining - Drilling holes in the monolith block to the specified tight tolerances $(1 \mathrm{~mm})$ is not possible using current technologies for a 1.5-m-length solid monolith block. The manufacturers may 
have to increase the web thickness to $2 \mathrm{~mm}$ or have larger tolerances than those specified by the current design. These larger webs and tolerances impose a severe core reactivity penalty (subcriticality). One solution is a larger core and higher uranium loading, which translates into a larger system footprint.

Another potential solution for the construction of the steel monolith, which avoids deep-channel drilling, is the application of Hot Isostatic Pressing (HIP) to pre-drilled plates. Plates with thicknesses on the order of 2.54-25.4 mm can readily be drilled with $1 \mathrm{~mm}$ webs with high accuracy. The plates would then be diffusion-bonded through the HIP process.

- Inspection and qualification - The monolith and heat pipes are integral to the design and will be required to meet and pass $100 \%$ inspection and validation requirements. If the monolith core is adversely affected either by the drilling of the fuel and heat pipe holes or the joining of the ends of the heat pipe to the monolith, the entire block must be scrapped and a new fabrication process started. The ability to perform inspections needed for the verification of welds and the performance of the heat pipes to meet design specifications is unknown.

- $\quad$ Monolith Structure - Survivability of the monolith to maintain structural integrity following a seismic event is of concern. The current design has the monolith placed in a horizontal configuration with much of the core weight $\left(\mathrm{UO}_{2}+\right.$ steel $)$ supported by the monolith thin steel webbings $(1 \mathrm{~mm}$ thickness between heat pipes and fuel pins and $1.75 \mathrm{~mm}$ thickness between fuel pins). It is unclear if the structure will maintain its geometry when exposed to an anticipated seismic loading. Because the reactivity control in the core is very sensitive to changes in its geometry, this could result in core slumping and possibly local power peaking, further challenging the integrity of the monolith and the ability to avoid localized power excursions.

Other concerns identified that will require additional development and understanding include:

\section{Core Criticality}

- Reactor core design is so finely optimized and the excess reactivity so small that even very small lattice pitch increases cause the core excess reactivity to drop precipitously. Web thicknesses, therefore, cannot be easily increased, fuel clad cannot easily be accommodated, and prefabricated heat pipes cannot be inserted directly into the steel monolith without significant reactivity loss and core re-design to avoid sub-criticality.

- Fast reactor U-235 nuclear reaction cross sections have uncertainties that lead to k-effective uncertainty on the order of the beginning-of-life excess reactivity.

Heat Pipe

- The ability to charge a heat pipe (potassium fluid and wick) following heat pipe weld to monolith is unknown.

- Thermal gradients are expected in the core and to be exacerbated by the localized loss of a heat pipe. The cumulative stress and strain introduced into the monolith segments and any resulting deformation or tear initiation in the monolith webbing are unknown. 
- Radioactivity release ex-core via heat pipe breach can emit activated potassium products: ${ }^{36} \mathrm{Ar}$ (269 years), ${ }^{42} \mathrm{Kr}$ (12.3 hours), and ${ }^{36} \mathrm{Cl}$ (301,000 years). Under the shell-and-tube heat exchanger concept, the activated products can be released directly to the environment in the exhaust air stream.

- Performance of the heat pipes under long-term irradiation and their ability to operate when exposed to fission products or contamination in the heat pipe is of concern. Impurity-induced corrosion has been identified as a potential life-limiting factor. Such age-related corrosion concerns can be mitigated with fabrication care and isolation from contamination sources. Agerelated mortality would be in large measure related to impurity corrosion or changes in surface chemistry driven by cumulative external contamination. Operating regimes, conditions, or properties that may lead to cascading heat pipe failures needs to be further explored and understood based on the configuration and operational lifetime.

\section{$\underline{\text { Monolith Structure }}$}

- At the elevated temperatures, the steel monolith enters a time-dependent material property regime. It is not clear if Section III Division 5 of the ASME pressure vessel code design rules can be met. These rules have not been vetted by the Nuclear Regulatory Commission (NRC). Reactor thermal transients may push steel temperatures higher yet, where material properties are not sufficient.

- Thermal gradients, thermal expansion, and thermal creep are expected at the prolonged elevated stainless steel temperatures $\left(650-700^{\circ} \mathrm{C}\right)$, which may cause the stainless steel monolith structure to flex or change shape under load and over time. Creep behavior of heat pipe welds and other structural welds at elevated temperatures is not known.

$\underline{\text { Welding }}$

- An automated welding technique will need to be selected - a technique that can make a large number of thin-wall welds on the monolith-heat pipe pressure boundary interface where physical access is very limited. Regardless of the welding technique, these welds will have to meet stringent quality assurance inspection standards and require careful design to eliminate, or minimize, the number of welds in high-temperature and high-stress regions. Is it possible to create thousands of welds successfully?

- Weld failure results in heat pipe failure and a potential pathway for activated potassium coolant and/or fission products release to the reactor containment and/or outside environment. Studies are needed to qualify the welding techniques and lifetime performance.

\section{Turbine Compressor}

- If the turbine pulls in foreign objects from the outside air or is damaged by natural disasters or deliberate attack, the objects may damage the blades to the point of creating additional shrapnel that is sent to the heat pipes/air heat exchanger. The shrapnel can potentially damages the heat pipes, which will release radioactive activation products into the atmosphere.

Additional details related to these design concerns are given in the reference [6] PIRT tables and the supporting neutronics, thermal/stress, and power conversion appendices. 


\section{DESIGN A}

Design A is the first of two INL alternative core design concepts for the Special Purpose Reactor. This section gives a description of the INL Design A concept along with a summary of some of the important neutronic, thermal, materials, fuel, and manufacturability features and characteristics to demonstrate the feasibility of the concept. More detailed preliminary supporting analyses can be found in the appendices.

\subsection{Description}

A cross-sectional view of the Design A core is shown in Figure 5. The active core retains the hexagonal shape of the LANL concept with the inner central void for the emergency shutdown rods. Radially, beyond the perimeter of the hexagonal active core, the reactor components and features will be essentially identical to those of the LANL Mega-Power concept. Only the active core is different. Design A replaces the LANL stainless steel monolith with individual fuel elements (Figure 6). The active core, as shown in Figure 5, holds 1,134 fuel elements, but there are additional open fuel element positions on the periphery of the core, as denoted by the green hexagonal dots in the figure. These additional fuel element positions can potentially add 72 more fuel elements. The additional fuel elements would displace alumina filler elements in these positions and add core reactivity, if needed.

The ability to add fuel elements to the core periphery provides two important benefits. First, core reactivity can easily be increased or decreased by simply adding or subtracting fuel elements. This option may be especially beneficial at initial core startup and criticality. If the core reactivity is determined to be low, fuel elements can be added. With a solid steel monolith core, adding fuel rods or heat pipes is not an option, since the monolith is fabricated with a fixed number of fuel and heat pipe channels. One sensitivity discovered in the assessment of the LANL concept was its high degree of optimization, such that core reactivity could be significantly impacted in a negative manner by small changes in lattice pitch, $\mathrm{UO}_{2}$ fuel pellet diameter, or enrichment. Because the beginning-of-life core excess reactivity in the LANL core is only approximately $\$ 2.88$, any small fabrication bias in the system parameters could negatively impact the excess reactivity and decrease the overall lifetime of the core. In Design A, increasing the inner clad thickness from the nominal $0.4 \mathrm{~mm}$ thickness to $>1.0 \mathrm{~mm}$ in order to reduce the thermal stress in the clad wall will significantly decrease core excess reactivity, thus making the addition of extra fuel elements a necessity.

The second benefit to being able to add fuel elements in the Design A core is the fact that additional heat pipes with each fuel element will increase the reactor cooling capability and average heat pipe thermal margin. In the nominal Design A concept, there are 1,134 heat pipes in the core, and each heat pipe must, on average, transport approximately $4.41 \mathrm{~kW}$ of power for the core operating at $5 \mathrm{MWt}$. This is "heat transport" is higher than in the LANL concept, which had 1,224 heat pipes, or $4.09 \mathrm{~kW}$ per heat pipe. Adding 72 peripheral fuel elements would bring the total number of heat pipes up to 1,206, or 4.15 $\mathrm{kW}$ per heat pipe, which is more in line with the LANL heat pipe average power.

Figure 6 shows the Design A fuel element. Essentially it consists of a heat pipe centrally located in the center of the fuel element. Surrounding the heat pipe is a $\mathrm{UO}_{2}$ fuel pellet clad on both radial surfaces with steel tubes. The $\mathrm{UO}_{2}$ fuel form will be similar to standard commercial fuel. The unique feature of the $\mathrm{UO}_{2}$ is the hexagonal shape of the pellet with an inner circular hole. Manufacture of these pellets is possible using standard techniques. The inner fuel cladding is a circular steel tube or pipe. The outer fuel 
cladding is a hexagonal stainless steel tube. These tubes are available commercially. The main advantages of the Design A fuel element are (1) it is manufacturable today, (2) materials are commercially available, and (3) each and every heat pipe and clad fuel rod can be individually fabricated, tested, inspected, and qualified prior to installation into the reactor core.

With the LANL monolith concept, the heat pipes are fabricated as part of the monolith. The fuel rods are drilled channels in the monolith that are filled with $\mathrm{UO}_{2}$ pellets and pressurized with helium gas; then steel end caps are welded to the monolith. Construction of the heat pipes and fuel rods revolves around a flawlessly-fabricated monolith structure. Although a workable fabrication technique could potentially be developed for the monolith heat pipes and fuel rods, verification testing and repair of a defective heat pipe or fuel rod channel could be difficult, possibly resulting in the replacement of the entire monolith structure. With a single fuel element design, as in Design A, a defective fuel element is easily detected and replaced.

For Design A, each heat pipe can then be fabricated ex-core in a dedicated factory with material testing and inspection of the stainless steel pipe, potassium metal, and wick. Assembly of heat pipe components and the filling of the heat pipe with liquid metal potassium and any required non-condensable gases can be done in a temperature- and pressure-controlled environment. As mentioned, every heat pipe can be inspected, tested, and qualified in a consistent manner. This individual heat pipe fabrication/qualification process should significantly boost the reliability of the heat pipes relative to the monolith concept. With the monolith concept, the heat pipes must be loaded with the wick and liquid metal and sealed after the heat pipe tubes are already integral to the monolith structure. Loading and sealing the 1,224 heat pipes in close proximity will be a challenge, but testing the heat pipes and repairing a defective heat pipe could be much more difficult.

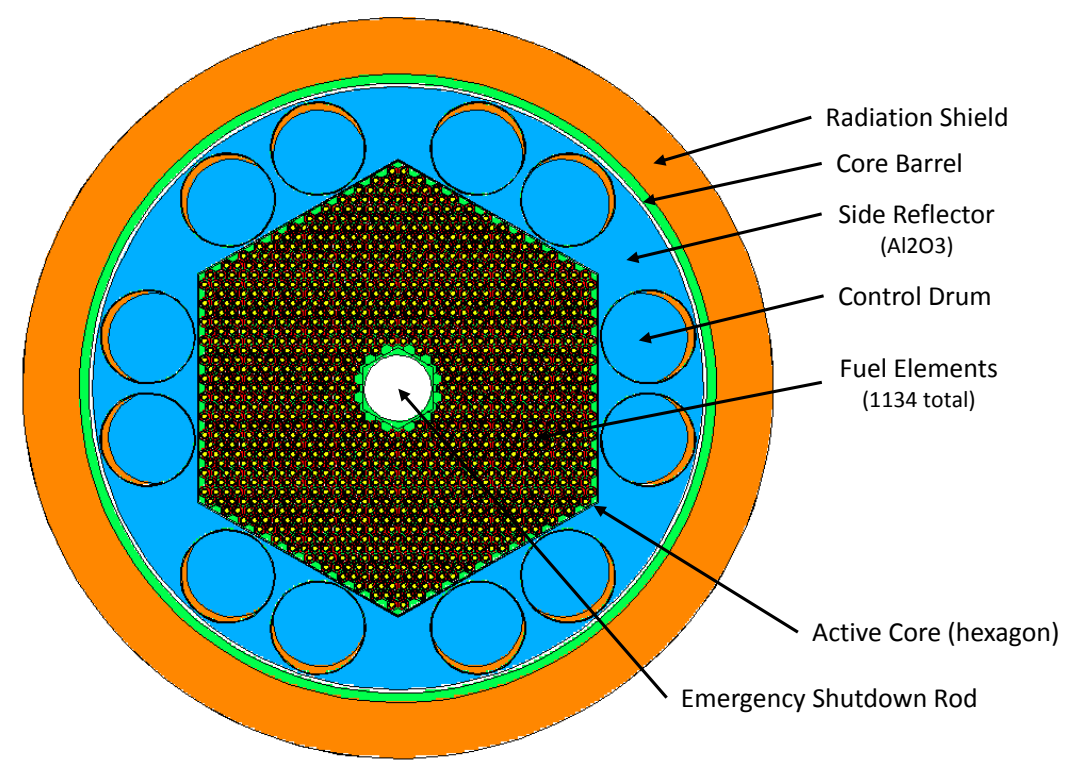

Figure 5. Cross-sectional view of the Design A core layout. 
For Design A, the cladding materials (circular and hexagonal Type 316 stainless steel pipes) for the fuel rod can also be inspected, cut, welded, and finished prior to loading the $\mathrm{UO}_{2}$ fuel pellets into the cladding. The $\mathrm{UO}_{2}$ pellets will be fabricated in a separate fuel fabrication plant and shipped to the fuel rod fabrication and assembly facility. The advantage of individually fabricated fuel rods is, again, similar to the individual fabrication of heat pipes, ease of fabrication, inspection, testing, and qualification.

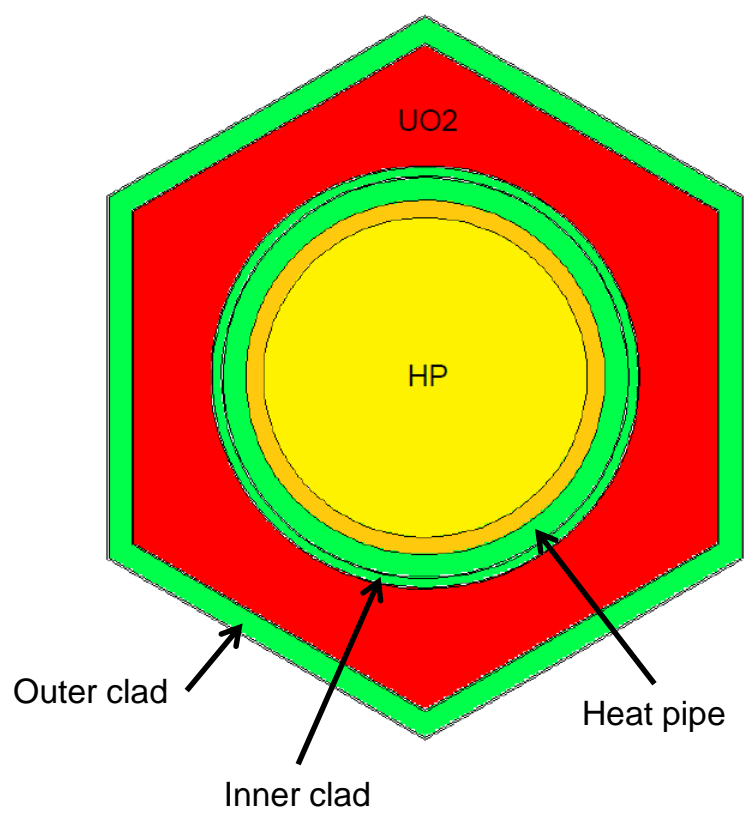

Figure 6. Cross-sectional view of a single Design A fuel element.

Figure 7 shows pertinent preliminary dimensions and materials associated with the Design A fuel element concept. The nominal fuel element flat-to-flat width, including the allowed gap between fuel elements in the core, is approximately $2.7862 \mathrm{~cm}$. This is also the fuel element pitch in the core. The gap between fuel elements is assumed to be $0.0064 \mathrm{~cm}$. The inner and outer diameters of the heat pipe are 1.575 and $1.775 \mathrm{~cm}$, respectively, assuming a wall thickness of $1.0 \mathrm{~mm}$. The heat pipe slides into the central hole of the fuel rod to assemble a fuel element. A gap of $0.0064 \mathrm{~cm}(2.5$ mils $)$ is provided between the heat pipe and inner fuel clad. This gap width is relatively small; a gap width of between 0.0102$0.0128 \mathrm{~cm}$ (4-5 mils) might be more practical in order to slide the heat pipe into the center of the fuel element.

There are a total of four gas gaps associated with the Design A fuel element (Figure 7). Each gap is a potential thermal barrier or thermal resistance that can elevate fuel, clad, and heat pipe wall temperatures. Some options are available to reduce the magnitude of these gap thermal resistances. For instance, the heat pipe and inner clad could be co-extruded, basically eliminating the gap (Gap 1). The two gas gaps between the $\mathrm{UO}_{2}$ and clad walls could be sized such that at operating conditions, the $\mathrm{UO}_{2}$ and stainless steel thermal expansion of the clad will close the gap and thereby minimize the thermal resistance of these two gaps (Gaps 2 and 3). Gap 4 could also be designed to close at operating temperature, such that adjacent elements would come into contact and provide a heat pathway in the event of a failed heat pipe. 
Gaps 1 and 4 could also be thermally bonded using a small amount of liquid metal potassium or sodium, although this would complicate the design.

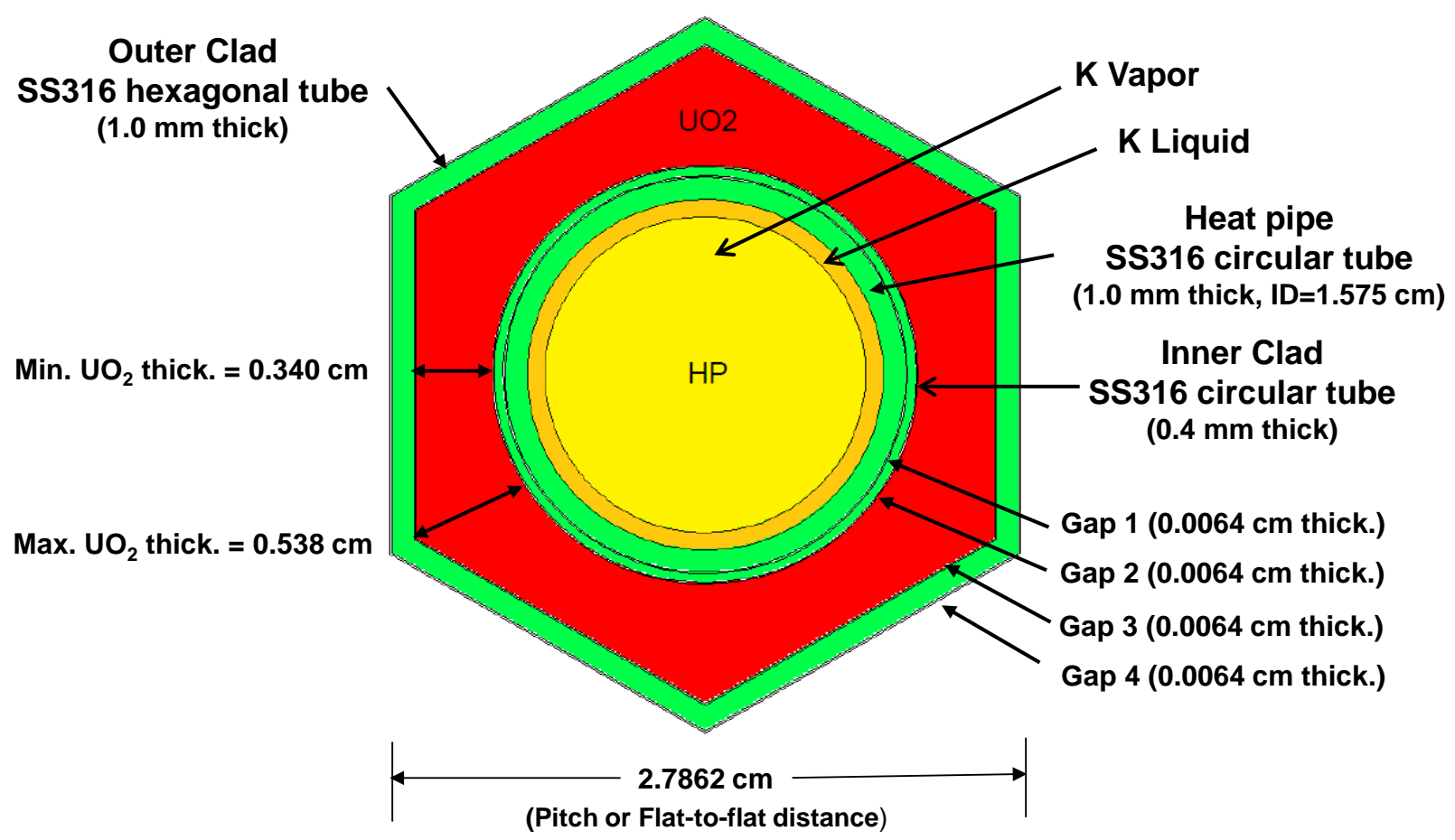

Figure 7. Nominal dimensions and materials for the Design A fuel element.

\subsection{Materials}

As in the LANL active core, Design A has the same six basic materials in the active core:

(1) Uranium dioxide $\left(\mathrm{UO}_{2}\right)$

(2) Potassium (K)

(3) Beryllium oxide $(\mathrm{BeO})$

(4) Alumina $\left(\mathrm{Al}_{2} \mathrm{O}_{3}\right)$

(5) Boron carbide $\left(\mathrm{B}_{4} \mathrm{C}\right)$

(6) Type 316 stainless steel (SS316)

$\mathrm{The} \mathrm{UO}_{2}$ is found in the fuel pellets. Potassium is the working fluid in the heat pipes. The beryllium oxide acts as a neutron reflector and multiplier in the upper and lower reflector above the $\mathrm{UO}_{2}$ fuel in the fuel elements. The $\mathrm{Al}_{2} \mathrm{O}_{3}$ is the side reflector material, and the $\mathrm{B}_{4} \mathrm{C}$ is the neutron poison in the control drums and shutdown rods. The SS316 is used in the heat pipe tubes, inner clad tubes, outer hexagonal clad tubes, upper and lower reflectors, and the core barrel.

Because the $\mathrm{UO}_{2}, \mathrm{BeO}, \mathrm{Al}_{2} \mathrm{O}_{3}$, and $\mathrm{B}_{4} \mathrm{C}$ are all high-temperature ceramic materials, the relatively low operating temperatures of the SPR $\left(500-800^{\circ} \mathrm{C}\right)$ pose no serious concerns for their usage in the active core. The fast neutron fluence in the SPR is small at less than two displacements per atom (dpa) and 
again, will pose no significant problem for these materials. $\mathrm{UO}_{2}$ has been tested up to very high burnups (>60,000 MWD/MTU), and since the SPR is expected to have very low burnups (2,000 MWD/MTU), or well below the commercial U.S. nuclear power reactor burnups, $\mathrm{UO}_{2}$ should function without issues in the SPR.

Type 316 stainless steel, on the other hand, is a metal and begins to lose physical strength at temperatures greater than $575^{\circ} \mathrm{C}$. Since the steel in the SPR is expected to operate at temperatures between 650 and $720^{\circ} \mathrm{C}$, use of SS316 in the SPR requires some attention. Section III, Division 5 of the ASME Boiler and Pressure Vessel Code contains design rules that are applicable for anticipated design conditions for the SPR. This section of the Code also specifies those materials that are allowed for nuclear construction, contains the required material properties for design and construction, specifies welding processes that are acceptable, and specifies inspection requirements. There is a very limited number of materials for which sufficient high-temperature properties for design are available. Type 304 and Type 316 stainless steel and Alloy $800 \mathrm{H}$ are qualified for Section III, Division 5 in the temperature range anticipated for this reactor. Time-dependent allowable stresses for Type 316 stainless steel are given for up to 300,000 hours. The design rules can be applied up to $800^{\circ} \mathrm{C}$.

Seamless and welded tube and pipe, forgings, plate, bar, and forged and bored pipe are allowed by the ASME Code and are widely available. Castings are not allowed; casting generally results in the formation of an additional phase in the material ( $\delta$ phase) that is susceptible to aging effects, including loss of ductility. Hot isostatically pressed power metallurgy products are allowed by a new Code Case. Additively manufactured components are not allowed. Material cold worked up to 5\% may be used in the cold-worked condition. Between 5 and $20 \%$ cold-worked material can be used up to 50,000 hours to a use temperature of $600^{\circ} \mathrm{C}$. For a longer amount of time, higher temperature or any high temperature application of material cold worked greater than $20 \%$, the component must be heat treated according to the appropriate specification prior to use.

Regardless of the final manufacturing method, it will be necessary to make a large number of in-core welds - many of which could be at a structural or pressure boundary. These may have to meet stringent reliability standards. Gas tungsten arc welding, shielded metal arc welding, and laser welding are allowed. Hot cracking is a potential issue with Type 316 fusion welds. Weld wire is typically specified with a chemistry different from base metal to form on the order of $10 \% \delta$ phase, which prevents cracking in the weld metal. This phase might need to be transformed by post-weld heat treatment. Autogenous welds (welding together two components of base material with no filler) typically do not have the proper composition to form $\delta$ phase and may suffer from weld cracking.

The maximum expected dose on the monolithic block in the LANL design is $1.9 \mathrm{dpa}$. At this level, irradiation hardening and embrittlement for wrought stainless steel have been extensively studied, and it does not appear to be an issue. In addition, reactions between Type 316 stainless steel and $\mathrm{Na}$ and $\mathrm{Na}-\mathrm{K}$ are well studied. There is also reactor experience with these systems, and no problems have been reported.

In contrast to both Type 316 and Type 304 stainless steel, Alloy $800 \mathrm{H}$ has higher allowable stresses at elevated temperatures. However, the irradiation resistance of this material has not been extensively studied, and there is no reactor experience with this alloy. Sodium compatibility has not been reported. There is also little operational experience with Type 304 stainless steel; the high-temperature allowable stresses are lower than either Type 316 stainless steel or Alloy $800 \mathrm{H}$. 
Sensitization is a well-known phenomenon in stainless steel during which the precipitation of Cr-rich carbides on the grain boundaries rob the adjacent areas of $\mathrm{Cr}$ and leave them susceptible to environmental effects. This phenomenon is of particular concern in light water reactors, because if the material is sensitized, it can be subject to stress corrosion cracking even in the absence of irradiation. In the case of the SPR, the operating temperature of the primary coolant is not high enough to cause sensitization, but weld metal or heat-affected zones of welds can be a problem. In addition to a microstructure that is susceptible to cracking, in order for stress corrosion cracking to occur, there must also be an applied stress and an appropriate environment. In light water reactors, the stress that causes cracking is often related to weldments, and it is the high-temperature reactor coolant water that gives rise to cracking. In the sodium cooled reactors that have been operated (e.g., fast reactors EBR II and FFTF), stainless steel that has grain boundary carbides has been used under stress; however, the environment has not been conducive to cracking, and no stress corrosion issues are reported.

In the high temperature reactor Code (ASME Section III, Division 5) both of the commonly used stainless steels, Type 304 and 316 are allowed for construction. Any high temperature reactor will operate in the temperature range where sensitization could occur. So the question is, if this is a well-known phenomenon and could be a problem, why are these materials allowed? This question was discussed at the August 2017 ASME Boiler Code Week in the Working Group on Allowable Stress Criteria. The effect of aging is considered by the Code, and the solution is to have a reduction factor applied to the yield strength and/or the tensile strength for the appropriate temperature range (see ASME Section III, Division 5, Table HBB-3225-2). For Type 316 stainless operated above $480^{\circ} \mathrm{C}$, there is a factor of 0.9 applied to the tensile strength and no reduction to the yield strength. This reduction to the tensile strength carries through to a reduction in the time-dependent allowable stresses in a manner specified in the Code. The principle use of the aging reduction factor is in earth-quake loading analysis. No one present at the Code Week discussion could recall that stress corrosion cracking of Type 316 in a sodium environment was an issue. In contrast to the light water experience, the sodium coolant does not cause embrittlement, even when the material has grain boundary carbide precipitation.

The Industrial Advisory Board recommended considering Type 310 or Type 347 stainless steel as an alternative to Type 316 stainless steel because the alternatives should be less prone to sensitization. Both of these alternative stainless steels are allowed in the ASME Code for some pressure vessel applications, but neither is allowed for elevated temperature nuclear construction. It is possible that they could be qualified for construction of elevated temperature nuclear components by establishing a Code Case for the material. Experience with the Alloy 617 Code Case at INL suggests that obtaining the required material performance data and balloting the Code Case would take longer than five years. A detailed assessment of these materials has not been completed; however, there is no known application in elevated temperature nuclear service and experience with these materials under irradiation. Like most of the austenitic stainless steels, the resistance of Type 310 and Type 347 stainless steel to environmental effects from the Na or $\mathrm{Na}-\mathrm{K}$ environment is likely adequate; however, there is no known operating experience in the liquid metal environment.

\subsection{Neutronic Analysis}

The neutronic characteristics of Design A are very similar to the LANL concept. The similarities, despite the geometric differences in the active core designs, are due primarily to the intentional emphasis 
INL placed on maintaining certain reactor parameters, such as the flat-to-flat dimensions, active core cross-sectional areas, and identical 1.5-meter fuel meat axial lengths. This created cores of near equal volume and $\mathrm{UO}_{2}$ mass, while leaving all other ex-core materials, dimensions, and components essentially the same. The beginning-of-life excess core reactivities between Design A and the LANL concept are consequently similar, or $\$ 3.82$ versus $\$ 2.93$ (Table 2). Other neutronic characteristics are also similar. Examples include the negative feedback coefficients of reactivity, control drum worth, emergency shutdown worth, neutron spectra, and core burnups. The reactor cores for Design A and the LANL concept will behave in similar fashion under steady-state and transient conditions.

Interestingly, the Design A core accommodates only 1,134 heat pipes, whereas the LANL concept can accommodate 1,224 in the core. Therefore, Design A has 90 fewer in-core heat pipes than the LANL concept. The cross sectional areas of the unit cells that compose the two cores are very similar, or 6.72 $\mathrm{cm}^{2}$ versus $6.65 \mathrm{~cm}^{2}$ for the Design A and LANL cores, respectively. This similarity in unit cell areas would imply that a nearly equal number of unit cells, and therefore heat pipes, should fit into the same available cross sectional core area. The somewhat subtle difference lies in the design differences between the two unit cells, or the geometrical arrangement of fuel and heat pipes in the unit cell. The difference prevents the placement 90 Design A fuel elements on the periphery of the core and in the center outside the emergency shutdown void. This is a consequence of fixing the outer core flat-to-flat dimension and the radius of the central emergency shutdown void. However, the more efficient packing of $\mathrm{UO}_{2}$ fuel in the Design A fuel element and the extra in-core space freed up from the elimination of the steel and void space between the six LANL core segments allows Design A to achieve a total $\mathrm{UO}_{2}$ core mass loading comparable to the LANL concept (5.19 MTU versus 5.22 MTU). The reduced number of heat pipes requires each heat pipe in Design A to lift, on average slightly more heat $(4.41 \mathrm{~kW})$ than the average heat pipe in the LANL concept $(4.08 \mathrm{~kW})$.

A nice feature of the Design A core concept is the ability to add additional fuel elements to the core periphery. In Figure 5, the green hexagonal dots or green cell positions on the core periphery are open positions that could accommodate additional fuel elements. The active core has nominally 1,134 fuel elements, but open positions allow for an additional 72 elements or a total of 1,206 fuel elements or heat pipes in the core. The additional fuel elements would displace alumina filler elements in these positions. Adding fuel elements gives the operator flexibility in adding extra core reactivity, if needed.

Radiation streaming from the heat pipes is a concern in all three SPR core designs and inherent in all heat pipe reactors. Accidental flooding of the heat pipes with light water is also a potential safety concern, possibly resulting from a transportation accident, in which heat pipes become damaged, allowing inleakage of water. Flooding several dozen heat pipes could lead to a super-critical core condition.

A potentially viable alternative to $\mathrm{UO}_{2}$ fuel is the metallic fuel form $\mathrm{U}-10 \mathrm{Zr}$. This fuel form is $90 \mathrm{wt} \%$ uranium metal and $10 \mathrm{wt} \%$ zirconium metal. The theoretical density of $\mathrm{U}-10 \mathrm{Zr}$ is higher than $\mathrm{UO}_{2}(16.0$ versus $\left.10.96 \mathrm{~g} / \mathrm{cm}^{3}\right)$, as is the uranium density $\left(14.40\right.$ versus $\left.9.66 \mathrm{~g} / \mathrm{cm}^{3}\right)$. The higher uranium density allows for a nearly $50 \%$ increase in uranium loading in the core, which can translate to either a smaller, more compact core or a lower fuel enrichment. For Design A, choosing a more compact core by reducing the number of fuel elements would also reduce the number of heat pipes in the core, placing a higher lift capacity requirement on each heat pipe. This would not be the first-choice solution. Instead, a reduction in the uranium enrichment would be more preferable. An enrichment reduction from $19.75 \mathrm{wt} \%$ to <15 wt $\%$ U-235 would be a significant and positive improvement in the Design A core. Another advantage of this metallic fuel is a higher thermal conductivity. Disadvantages include fuel swelling, fission gas release, 
fuel redistribution, lower melting point $\left(1,160^{\circ} \mathrm{C}\right)$, and cladding interactions; however, since the burnup in these SPR cores is so small, the effects of fuel swelling due to fission gas production would be minimal. The deliberate introduction of porosity into the metallic fuel would, therefore, also be small, and near fulldensity metallic fuel might be possible. Introduction of a metallic fuel form, such as U-10Zr, into the Design A fuel element to replace the $\mathrm{UO}_{2}$ ceramic fuel form appears to some very have positive attributes.

For a more complete look at all the preliminary neutronic analysis of Design A, see Appendix A. Appendix A gives additional neutronic parametric studies and results, in addition to computer model descriptions, modelling assumptions and techniques, and computer code software and tools. Comparisons to the LANL concept are also given.

\subsection{Thermal Analysis}

A preliminary $5 \mathrm{MWt}$ thermal analysis has been performed for Design A under normal steady-state operating conditions. Table 3 gives the peak temperatures calculated at various locations within a single Design A fuel element, and for comparison purposes, calculated peak temperatures for the LANL concept [6] are also given. For the LANL concept, two sets of peak temperatures are presented, one for temperatures calculated assuming an isothermal heat pipe wall temperature of $677^{\circ} \mathrm{C}$ per reference [6], and a second set of temperatures (in parentheses) adjusted to an isothermal heat pipe wall temperature of $712.5^{\circ} \mathrm{C}$. This provides a more one-to-one comparison between the Design A and LANL concepts. The higher isothermal temperature of the heat pipe wall is based on more detailed heat pipe calculations performed after the publication of reference [6].

The peak $\mathrm{UO}_{2}$ fuel temperatures for Design A and the LANL concept are similar in magnitude at around $770-790^{\circ} \mathrm{C}$. These fuel temperatures are: (1) relatively low compared to large power reactors with maximum fuel temperatures ranging from $1,700-2,200^{\circ} \mathrm{C}$ [7], (2) considerably below the $2,800^{\circ} \mathrm{C}$ melting point of $\mathrm{UO}_{2}$, and (3) substantially below the $1,027^{\circ} \mathrm{C}(1,300 \mathrm{~K})$ temperature threshold where fission product gases begin to nucleate, grow, and diffuse [9] out of the $\mathrm{UO}_{2}$ fuel matrix. Combined with the low fuel burnup, fission product gases are expected to be mostly confined to the $\mathrm{UO}_{2}$ matrix, thereby reducing the need for a large fission gas plenum in the Design A fuel element and the LANL monolith. Contamination of the helium fill gas with fission product gases $(\mathrm{Kr}$ and $\mathrm{Xe})$ in the gas gaps between the $\mathrm{UO}_{2}$ and the Design A clads or the LANL monolith is not a major concern. Thermal stress in the $\mathrm{UO}_{2}$ fuel may produce some cracking in the ceramic at startup, but it is not expected to a major concern, either.

Understanding the magnitude of the thermal stresses induced in the steel heat pipe wall and the adjacent inner clad are of high importance for Design A. One of the goals of Designs A and B was to have thermal stresses in the in-core steel to be less than the excessive thermal stresses calculated for the LANL design [6]. A single failed heat pipe in the LANL design sent the thermal stresses to exorbitant levels.

In Design A, the steel heat pipe and inner clad walls separate the hot $\mathrm{UO}_{2}$ fuel from the heat sink (potassium vapor). It is within these two steel walls that the largest thermal gradients are expected to exist

in a Design A fuel element. The two-dimensional thermal model predicts a maximum thermal stress in the inner clad of approximately 4.5 MPa which is a factor of 8 less than the $37.1 \mathrm{MPa}$ maximum stress calculated in the steel monolith [6]. The two-dimensional model, however, does not include threedimensional effects from axial volumetric heat rate variations in the fuel or fuel element end-effects in the 
grid plates. The end-effects are, however, expected to be limited due to the ability of individual fuel elements to move freely in the axial direction and not be constrained at the top or bottom of the core. Future three-dimensional analysis will determine the final thermal stress conditions, but for now the much lower two-dimensional stress results are a positive indication.

Although the outer clad temperatures are higher than the inner clad temperature, thermal stresses in the outer clad are expected to be relatively inconsequential. Heat flow between adjacent fuel elements is small; therefore, adjacent outer clad temperatures should be near isothermal, creating essentially a semiadiabatic boundary condition around each fuel element. Thermal gradients across the outer steel clads are small and relatively inconsequential, as are the thermal stresses.

Table 3. Peak temperatures calculated for Design A and the LANL concept.

\begin{tabular}{|c|c|c|c|}
\hline \multicolumn{2}{|c|}{ Design A } & \multicolumn{2}{c|}{ LANL Concept } \\
\hline $\mathrm{UO}_{2}$ fuel & $768^{\circ} \mathrm{C}$ & $\mathrm{UO}_{2}$ fuel & $753^{\circ} \mathrm{C}\left(789^{\circ} \mathrm{C}\right)$ \\
\hline Outer clad & $765^{\circ} \mathrm{C}$ & Monolith & $696^{\circ} \mathrm{C}\left(731.5^{\circ} \mathrm{C}\right)$ \\
\hline Inner clad & $716^{\circ} \mathrm{C}$ & & \\
\hline $\begin{array}{c}\text { Heat pipe wall } \\
\text { (isothermal) }\end{array}$ & $712.5^{\circ} \mathrm{C}$ & $\begin{array}{c}\text { Heat pipe wall } \\
\text { (isothermal) }\end{array}$ & $677^{\circ} \mathrm{C}\left(712.5^{\circ} \mathrm{C}\right)$ \\
\hline
\end{tabular}

The maximum thermal stress in the LANL concept has been calculated to be $37.1 \mathrm{MPa}$ at $696^{\circ} \mathrm{C}$ [6]. This peak stress level was calculated in the thin steel webbing between two fuel pellet stacks in the monolith. The ASME Code, at a temperature of $700^{\circ} \mathrm{C}$, designates the maximum allowable stress to be only 29.6 MPa. Exceeding the apparent allowable ASME Code stress level under normal steady-state reactor operating conditions has been a major concern and an impetus for INL to produce alternative active core designs to replace the monolith.

Appendix C provides more detail on the preliminary Design A thermal analysis models, codes, input data, and analysis results.

\subsection{Heat Pipe Analysis}

Design A heat pipe nominal dimensions and characteristics are based on the preliminary heat pipe design provided by LANL heat pipe experts. Table 4 gives the LANL heat pipe dimensions and characteristics. One key difference is that the cores of both Designs A and B core will be vertically oriented; therefore, the heat pipes will be as well. The LANL concept operates with the core and heat pipes in a horizontal orientation. With vertical orientation, the heat pipes will gain the added assist of gravity.

The Table 4 specification data has been used as input data into the INL version of the HTPIPE code [10]. INL's HTPIPE code has been preliminarily verified against other heat pipe codes, with published performance results. Very good agreement was obtained between the INL HTPIPE code and these other 
heat pipe codes. The agreement has provided a certain level of confidence in the HTPIPE-calculated results given here for Design A.

Table 4. Design A nominal heat pipe dimensions and characteristics.

\begin{tabular}{|l|l|}
\hline Heat pipe orientation & Vertical \\
\hline Working liquid metal fluid & Potassium \\
\hline Operating temperature range & $\begin{array}{l}627-727^{\circ} \mathrm{C} \\
(900-1,000 \mathrm{~K})\end{array}$ \\
\hline Operating power range (kW) & $3.5-7.5$ \\
\hline Overall length (m) & 4.0 \\
\hline Evaporator length (m) & 1.5 \\
\hline Adiabatic length (m) & 0.3 \\
\hline Condenser length (m) & 2.1 \\
\hline Inner pipe diameter (cm) & 1.575 \\
\hline Outer pipe diameter (cm) & 1.775 \\
\hline Stainless pipe wall thickness (mm) & 1.0 \\
\hline Wick type & Annular gap \\
\hline Annulus thickness (mm) & 0.7 \\
\hline Screen thickness (mm) & 1.0 \\
\hline Screen material & 0.706 \\
\hline Screen mesh size & 0.015 \\
\hline Effective pore radius (mm) & Stainless steel \\
\hline Porosity & (mesh \\
\hline
\end{tabular}

As an example, using the heat pipe specifications given in Table 4, the Design A heat pipe is calculated to have a lift capacity of between 3.5 and $7.5 \mathrm{~kW}$ per heat pipe over the $900-1,000 \mathrm{~K}$ temperature range. At $950 \mathrm{~K}\left(675^{\circ} \mathrm{C}\right)$, the lift capacity is approximately $5.0 \mathrm{~kW}$.

The nominal core design for Design A calls for 1,134 heat pipes, or one heat pipe per fuel element. At $5 \mathrm{MWt}$, each pipe is expected to lift on average $4.41 \mathrm{~kW}$, or approximately $8 \%$ more than the $4.08 \mathrm{~kW}$ per pipe required for the LANL concept, which uses 1,224 heat pipes. Despite the slightly higher average power lift requirement for Design A, this does not appear to be a problem, mainly because the increase is relatively small and a sufficient margin exists to accommodate powers up to $7.5 \mathrm{~kW} /$ pipe.

Several good options are available to improve the overall heat pipe capacity of the heat pipes and to boost the operating margin. These options include: 
- Increasing the inner diameter of the heat pipes,

- Orienting the heat pipes vertically (for gravity assist) instead of horizontally,

- Adding fuel elements on the core periphery,

- Use of an advanced wick design.

Option 1: Increasing the inner diameter of the heat pipes is a simple solution to boost lift capacity and operating margin. However, the only way to effectively increase the heat pipe inner diameter and maintain core reactivity is to allow the core footprint to increase slightly, which translates into an increase in the individual fuel element pitch (or fuel element flat-to-flat dimension). The pitch increase can allow more U-235 in each element, and therefore, an increase in the overall U-235 core mass. The additional U235 mass helps offset the negative core reactivity introduced by the larger-diameter heat pipe and pitch (increased neutron leakage). As an example, if the inner diameter of the heat pipe is increased from the nominal $1.575 \mathrm{~cm}$ to $1.975 \mathrm{~cm}$, or a $4.0 \mathrm{~mm}$ diameter increase, the operating margin could be increased from approximately $7.5 \mathrm{~kW}$ to $17.0 \mathrm{~kW}$ per heat pipe before hitting the capillary limit. The core flat-toflat dimension would increase from $0.994 \mathrm{~m}$ to $1.108 \mathrm{~m}$, and the U-235 mass would increase from $904 \mathrm{~kg}$ to $1,050 \mathrm{~kg}$, or an increase of $147 \mathrm{~kg} \mathrm{U}-235$. This is not a burdensome change for a substantial gain in heat pipe performance.

On the other hand, if the fuel element pitch is held constant (core footprint maintained at $0.994 \mathrm{~m}$ flat-toflat) and the heat pipe inner diameter is increased, the 1,134-element core will go subcritical ( $k$ effective $=0.93479$ ) for just a $2.0 \mathrm{~mm}$ increase in the inner diameter of each heat pipe. In this case, the increase in heat pipe diameter comes at the expense of the $\mathrm{UO}_{2}$ fuel, a $200 \mathrm{~kg}$ loss of U-235 in the core. The loss of fuel and an increase in neutron leakage produces two negative core reactivity losses that result in a sub-critical core with all control poisons out of the core.

Option 2: The second option, or vertical orientation of the heat pipes, is already planned for the Design A concept. Wicked heat pipes can operate in any orientation from vertical to horizontal, and even inverted. Under certain operating conditions, heat pipes may actually perform better in the horizontal mode. For Design A, however, the vertical orientation is preferred in order to take advantage of the gravity force that can assist the capillary force in returning condensed liquid potassium to the lower-elevation evaporator section of the heat pipes. At $950 \mathrm{~K}\left(675^{\circ} \mathrm{C}\right)$, the lift capacity is calculated to be approximately $5.0 \mathrm{~kW}$ with an operating margin of 7-8 $\mathrm{kW}$. If the heat pipes were horizontally oriented, the operating margin would only be about $3.0 \mathrm{~kW}$.

Another advantage of a vertically-oriented core (fuel elements and heat pipes) is that the weight of each individual fuel element is now supported by the lower grid plate support structure, and not by other fuel elements. This helps to alleviate additional mechanical loads placed on the fuel cladding and heat pipe steel structures. In the horizontally-oriented LANL concept, the monolith segments on the bottom of the core will experience additional stress loads due to the weight of the monolith structure and fuel above. A second advantage of vertical core orientation is reliable core shutdown using gravity-driven emergency shutdown control rods.

Option 3: The third option, adding fuel elements to the core periphery, for Design A only, is an option to boost core reactivity, if needed. As previously mentioned, adding fuel elements adds a corresponding number of additional heat pipes as well; the extra heat pipes will reduce the average heat pipe load. Design A can use alumina-filled elements on the periphery of the core as place holders for additional fuel 
elements. Neutronic results show, however, that simply adding additional fuel elements to the periphery of the core is not a great solution to counter the loss of reactivity introduced by increasing the heat pipe inner diameters. Small increases in the inner diameter of the heat pipe significantly reduces core reactivity. The addition of low-importance (neutronically) fuel elements on the periphery of the core will require a substantial number to create a noticeable amount of extra core reactivity. As an example, for a small $2.0 \mathrm{~mm}$ increase in the inner heat pipe diameter, 702 additional peripheral fuel elements would compensate only half of the lost core reactivity. The active core footprint would also increase. The flat-toflat dimension would expand from $1.04 \mathrm{~m}$ to $1.22 \mathrm{~m}$. This option is feasible, but not very practical for this application.

Option 4: The fourth option of implementing an advanced wick design is probably the best option to improve heat pipe operating margin. LANL heat pipe experts are currently working on new cutting-edge wick technology to improve the heat pipe performance. Wick design details are not yet available, but preliminary heat pipe code predictions indicate potentially very large increases in the operating margin may be possible. Using an advanced wick design is probably the best option, since no penalty is incurred for core size or weight.

It must be pointed out that the current heat pipe specification (Table 4) will deliver acceptable heat removal capability for the Design A concept under normal operating conditions. The same is true for the LANL and Design B concepts as well. A higher operating margin is, however, always a more desirable performance goal and an advanced wick design would make this possible.

For a more complete and detailed heat pipe analysis of the heat pipes for the SPR concepts, see Appendix E.

\subsection{Manufacturability}

The Design A fuel element is composed of three basic components: (1) the unique hexagonal-shaped $\mathrm{UO}_{2}$ fuel pellet with a central circular hole, (2) the inner clad (circular stainless steel tube), and (3) the outer clad (hexagonal stainless steel tube). The manufacture of the uniquely shaped $\mathrm{UO}_{2}$ pellet is possible using a least three different techniques. Selection of the most appropriate technique and optimizing the process to produce pellets that meet specifications will, however, require some experimentation. The three techniques are explored in the following sections.

Manufacture of the circular and hexagonal cladding tubes is possible today using existing commercial vendors. There are a variety of U.S. commercial suppliers of steel tubes. A few of these suppliers include: (1) Century Tube Corporation, (2) The Sandvik Group (Sandvik Materials Technology), (3) Boiler Tube Company of America, (4) Sumitomo Corporation, (5) PCC York, (6) ATI Metals, (7) Curtiss-Wright, and (5) Louisiana Steel. These companies have the capability to manufacture welded or seamless steel tubing in both standard and custom cross section shapes with a variety of materials, including Type 316 stainless steel. These U.S. vendors can also provide design and manufacturing expertise, as can the SPR Industrial Advisory Board members.

With regard to the outer clad hexagonal tube, a concern was raised during the SPR Industry Advisory Board review meeting. The concern had to do with the flatness of the hexagonal tube along its length. The tubes have a relatively large aspect ratio of 50:1 (length-to-width) and extrusion of the tubes, welding of 
end fittings, and accommodation of the central heat pipe may impact the tube flatness. Flatness is needed for ease of core assembly and a tight compact array of fuel elements in the core.

The preferred method for fabrication of the heat pipes is to fabricate them individually, and then slide the heat pipe into the center of the fuel element. Co-extrusion of the heat pipe tube and the inner clad tube is also an option that is being evaluated now. Co-extrusion will not only eliminate the thermal resistance gap (Gap 1) between heat pipe and the inner clad, but would also eliminate the tolerance specification required between the two tubes, which could potentially simplify the manufacturing and assembly process. This option will need to be explored later.

\subsection{Fuel Fabrication Pathways}

Uranium dioxide $\left(\mathrm{UO}_{2}\right)$ is currently the primary fuel choice for Design A due to its wide usage today in commercial power reactors in the U.S. and around the world. The associated high technology readiness level is very attractive and would align well with an aggressive 7-year SPR deployment schedule. This section discusses the $\mathrm{UO}_{2}$ fabrication techniques available at the INL; techniques that can be implemented to support a small research and development effort to fabricate the design A hexagonally-shaped $\mathrm{UO}_{2}$ pellets. In addition, the fabrication of metallic fuel in the form of either $95 \% \mathrm{U}-5 \%$ Fs (Fissium) or $90 \% \mathrm{U}$ $10 \% \mathrm{Zr}(\mathrm{U}-10 \mathrm{Zr})$ alloys is also discussed, as there are reactivity benefits to be gained with a higher density fuel form.

In selecting a fuel system composition, it is important to consider the operational characteristics and performance requirements of the reactor. Additionally, Design Basis Accidents (DBAs) and Beyond Design Basis Accident (BDBA) event conditions must be factored into fuel materials selection. For installations where maximum security can be unequivocally maintained, the selection of a fuels system can be based on the operational performance, DBA and economics of production. However, for systems that are considered for general purpose and transportable to theaters of war such as forward deployment military bases, consideration of all other conceivable external threats to the integrity of the reactor system must be made in the design of the fuel system. Specifically, fuels should minimize the release of fission products during high temperature excursions that may arise from deliberate overpower of the reactor (if not inherently protected by system design) and in the event that all heat removal mechanisms are made inoperable by mechanical cutting or explosive means, either during reactor operation or immediately after reactor shutdown from operational power.

This section on fuel fabrication pathways specifically written to the Special Purpose Reactor is excerpted largely from Reference [11].

\subsection{1 $\mathrm{UO}_{2}$ Fuels}

\section{$\mathrm{UO}_{2}$ Powder feedstock}

Commercial $\mathrm{UO}_{2}$ is produced in the United States at an enrichment of $5 \%{ }^{235} \mathrm{U}$ or less. Federal service vendors such as BWXT in Virginia are capable of supplying $\mathrm{UO}_{2}$ at higher enrichments; however, the cost of supply may be too great for research and single element prototype experiments. For this reason, production at the Department of Energy complex may be most feasible. 
The INL currently has no feedstock of enriched $\mathrm{UO}_{2}$ beyond commercially produced materials $(<5$ $\%{ }^{235} \mathrm{U}$ ), thus for lab scale feasibility studies to produce LEU fuel elements at approximately 19.75 $\%{ }^{235} \mathrm{U}$, enriched $\mathrm{UO}_{2}$ will have to be prepared. The commercial preparation of $\mathrm{UO}_{2}$ proceeds through either a wet or dry process starting with $\mathrm{UF}_{6}$. Uranium hexafluoride is converted to $\mathrm{UO}_{2} \mathrm{~F}_{2}$, followed by conversion to ammonium diuranate (ADU) or ammonium-uranyl-carbonate (UAC). ADU or AUC is then calcined to form $\mathrm{UO}_{3}$, which can then be reduced to $\mathrm{UO}_{2}$. This process for enriched $\mathrm{UO}_{2}$ can be performed by Y-12, although at significant cost, likely prohibitively expensive for a lab scale feasibility study.

Purchasing a reaction intermediate, such as ADU, and completing the conversion to $\mathrm{UO}_{2}$ at INL, may allay some of the cost. Conversion of ADU would proceed by calcining at $600^{\circ} \mathrm{C}$ for 2.5 hours, after which time a flow of nitrogen/hydrogen/steam would be maintained for 90 minutes. After ending the gas flow, the temperature would be stepped down to room temperature, and the enriched $\mathrm{UO}_{2}$ powder can be collected. Although the cost will be reduced, there is still a significant cost for ADU from Y-12.

Another possible method to prepare $\mathrm{UO}_{2}$ is to start with uranium metal. This would likely be less efficient than the $\mathrm{UF}_{6}$ process, but for a lab scale study this may be the preferred method. The INL has the feedstock, so no material would have to be purchased. The first step is to prepare the desired enrichment by mixing depleted and enriched uranium. This could easily be performed in an arc-melter. The next step is dissolution in nitric acid, as shown in reaction 1, producing the water-soluble uranyl nitrate hexahydrate. This step is followed by reaction with aqueous ammonia to produce ADU [12]. The byproduct in reaction 1 is shown as $\mathrm{X}$ since the composition can vary, depending on nitric acid concentration used. A mix of $\mathrm{NO}_{\mathrm{x}}$ compounds will be formed. Due to this the reaction is not balanced. In reaction 2, ADU is shown as with a specific formula, although the exact composition can vary.

$\mathrm{U}_{(\mathrm{s})}+\mathrm{HNO}_{3}+6 \mathrm{H}_{2} \mathrm{O} \rightarrow \mathrm{UO}_{2}\left(\mathrm{NO}_{3}\right)_{2} \cdot 6 \mathrm{H}_{2} \mathrm{O}+\mathrm{X}$

$\mathrm{UO}_{2}\left(\mathrm{NO}_{3}\right)_{2} \cdot 6 \mathrm{H}_{2} \mathrm{O}+6 \mathrm{NH}_{4} \mathrm{OH} \rightarrow\left(\mathrm{NH}_{4}\right)_{2} \mathrm{U}_{2} \mathrm{O}_{7}+4 \mathrm{NH}_{4} \mathrm{NO}_{3}+3 \mathrm{H}_{2} \mathrm{O}$

In reaction 2, ADU precipitates from the solution. After separation, the final conversion to $\mathrm{UO}_{2}$ can be carried out as described above, i.e. calcining followed by nitrogen/hydrogen/steam treatment.

This method of producing $\mathrm{UO}_{2}$ appears simple, especially when compared to the method using $\mathrm{UF}_{6}$ as a feedstock. Commercially this would not be viable, though, due to the slow dissolution rate of $U$ metal in nitric acid. In a lab scale feasibility study, commercial quantities are not needed, making this method more attractive. The dissolution rate will likely never be fast, but there are methods to dissolve the metal faster [13]. Heating the solution to modest temperatures, such as $50^{\circ} \mathrm{C}$, will increase the rate, as will addition of $\mathrm{KNO}_{2} . \mathrm{HNO}_{2}$ is believed to catalyze the reaction, thus addition of the salt, forming $\mathrm{HNO}_{2}$ in solution, will increase the dissolution rate. An obvious method to increase dissolution is to decrease surface area of the material. Breaking large ingots into small pieces will increase dissolution, but the pieces must not be a powder. Adding uranium powder to nitric acid can be explosive.

Dissolution is the best method to ensure complete mixing of depleted and enriched $\mathrm{UO}_{2}$, thus for either production method, the desired enrichment, i.e. 19.75\%, needs to be used in the feedstock from the 
beginning. Trying to blend depleted and enriched $\mathrm{UO}_{2}$ powder is limited by particle size. A truly homogenous mix, at an atomistic level, can only be obtained through dissolution.

The INL has facilities that could accommodate the production of $\mathrm{UO}_{2}$ of selectable enrichment. The ZPPR warehouse (MFC-784) at MFC is a possibility, as is CPP-1634 at INTEC for conversion to $\mathrm{UO}_{2}$ from $\mathrm{ADU}$. If the dissolution method for conversion of uranium metal to $\mathrm{UO}_{2}$ is selected, wet chemistry laboratories, such as RCL (MFC-1702) at MFC, or CFA-625 at the Central Facilities, may provide appropriate accommodation.

\section{$\mathrm{UO}_{2}$ Dopants for FCCI protection}

Recent advances in oxide fuel system performance have been made, specifically in the reduction of FCCI and related failures in LWR fuels. These advances have been made by adding dopants such as $\mathrm{Cr}_{2} \mathrm{O}_{3}$ [14] within the oxide fuel which allows for rare earth, lanthanide fission products to complex with the added species (e.g. Cr), thereby pacifying chemically aggressive species before their interaction with the cladding. 3-8 wt\% additive concentration is typically sufficient to provide FCCI protection and has been demonstrated in commercial LWR fuel operation [14][15].

\section{Spark Plasma Sintering of $\mathrm{UO}_{2}$ and doped $\mathrm{UO}_{2}$.}

Spark plasma sintering has been developed and demonstrated for sintering of $\mathrm{UO}_{2}$, doped $\mathrm{UO}_{2}$ and other composite uranium fuel meats. The SPS process is net shape and can produce a fuel meat required by the HPR design with a sintering schedule of 1-5 minute hold time at $1550 \mathrm{C}$. The INL RSPS at MFC 784 could produce 7-10 inches of fuel meat length per day assuming a single shift. The RSPS will be able to process the required enrichment of fuel in MFC 784. To date, $\mathrm{UO}_{2}$ has been pressed into round pellets, some with an intricate annulus, embedded thermal conductor or plain pellet with a dish and chamfer. The maximum $\mathrm{UO}_{2}$ pellet diameter that has been tried to date was $40 \mathrm{~mm}$. Therefore the HPR geometry should be feasible given experience in metal fuel fabrication using SPS.
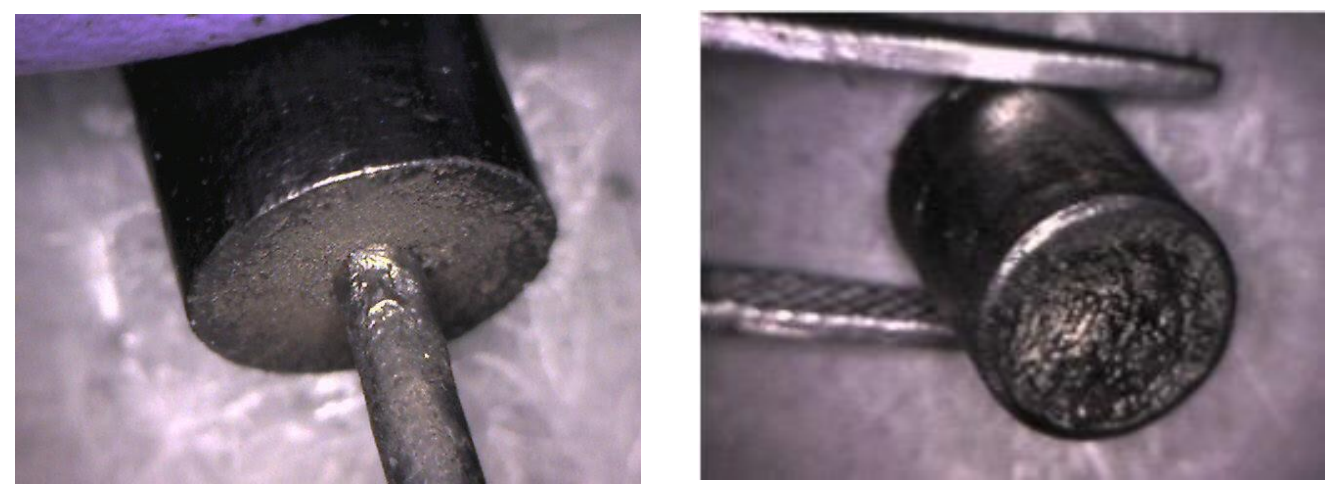

Figure 8: Example ceramic fuel pellets fabricated via SPS. (Left) $\mathrm{UO}_{2}$ pellet with axial Niobium thermal conductor. (Right) net-shaped ceramic pellet with commercial PWR geometry, dish and chamfer.

\section{Isostatic Bag pressing of $\mathrm{UO}_{2}$ and doped $\mathrm{UO}_{2}$}

Isostatic Bag pressing has been used industrially to rapidly mass-produce ceramic parts and components. The automotive industry uses isostatic bag pressing to produce ceramic insulators for spark 
plugs. Recent R\&D at INL in collaboration with the equipment vendor, Loomis, has produced annular ceramic pellets. The INL currently owns an isostatic bag press, but neither Loomis nor INL have produced $\mathrm{UO}_{2}$ components. To date $\mathrm{CeO}_{2}$ has been used as a surrogate with good results. The INL's equipment is currently out of service in storage but could be re-commissioned within a 6-12 month period at MFC-784.

Isostatic bag pressing is a powder processing technique that uses a flexible polymer bag that is shaped to the basic geometry required for the finished part. Bags may be 10-12 inches in length and are loaded with powdered material. Bags may be heat sealed to help with contamination control. The process is rapid once the polymer bags are filled with the powder to be consolidated: 10-12 inches of 'green' fuel meat could be produced every 1-2 minutes (3,240 inches per day). Once compacted the green fuel meat must be sintered in a controlled atmosphere furnace. Current furnace capacity would allow one green compact (10-12 inches in length) to be sintered per day at MFC EFF. Similar heat treatment throughput is anticipated in FASB. With the purchase of a new controlled atmosphere furnace for MFC 784, it would be possible to process up to 600-1200 inches per day, subject to a criticality safety design review.

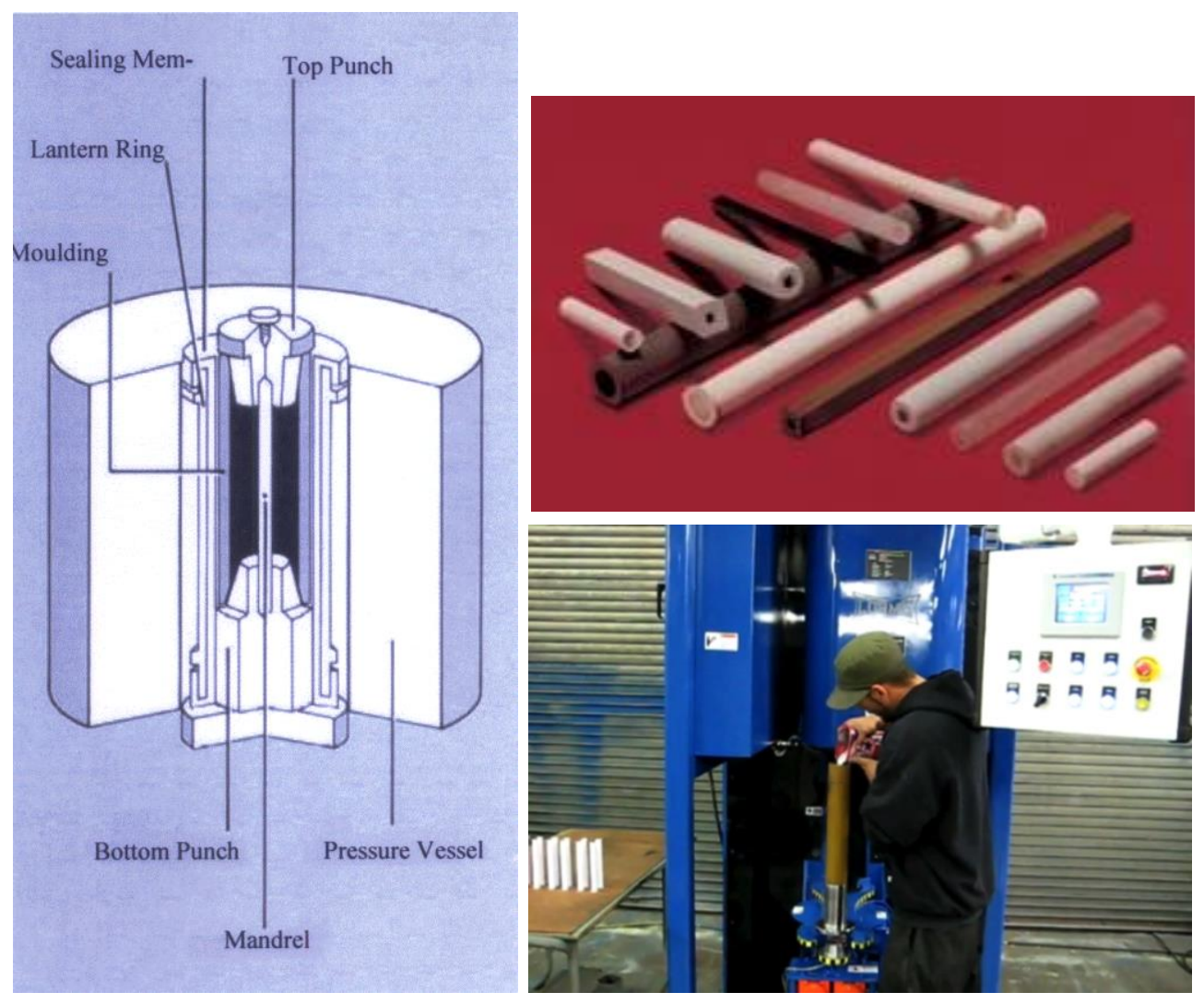

Figure 9: (Left) Isostatic Bag Press apparatus shematic. (Top Right) example rods and other net shapes made using the process. (Bottom Right) photograph of the INL Isostatic Bag Press in operation with $\mathrm{CeO}_{2}$ as a surrogate for $\mathrm{UO}_{2}$.

An overview of the process for isostatic bag pressing is provided in Figure 8 below: 

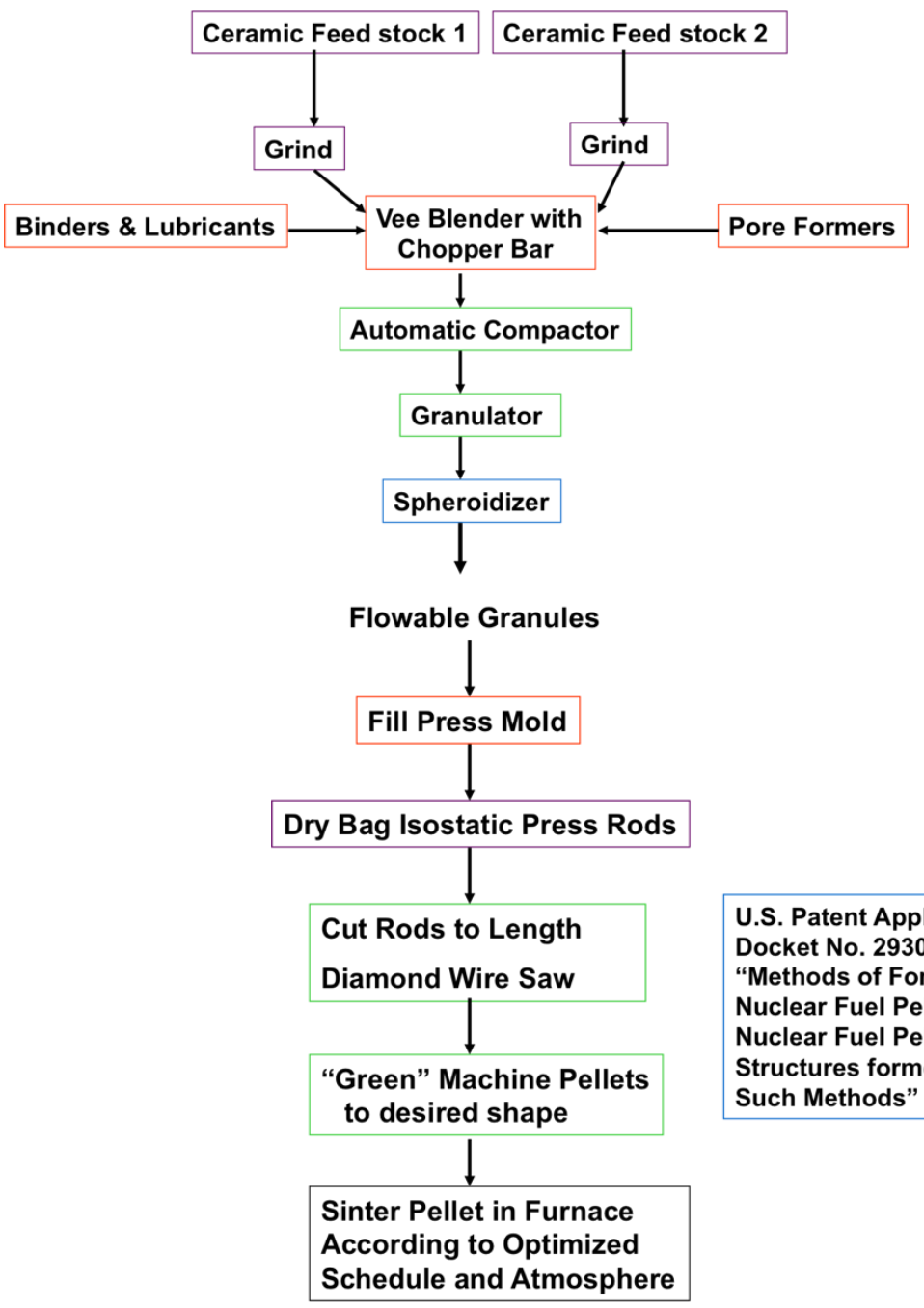
U.S. Patent Application Docket No. 2930-9318 US "Methods of Forming Nuclear Fuel Pellets, and Nuclear Fuel Pellets and Structures formed by Such Methods"

Figure 10: Process flow sheet for production of suitable ceramic fuel meat via isostatic bag pressing.

Hot Isostatic pressing of $\mathrm{UO}_{2}$ and doped $\mathrm{UO}_{2}$.

The INL has a hot isostatic press at MFC FASB that is capable of producing sub-length uranium oxide fuel slugs ( $\sim 10$ " length) for the HPR design. HIP would also require the post fabrication machining to remove the HIP can from the outside of the part. EDM or mechanical machining can again be used to perform this work. Post sinter heat-treatment is not likely to be required since the HIP process is performed in a sealed can/system. Therefore, it is anticipated that $\mathrm{UO}_{2}$ feedstock would yield a $\mathrm{UO}_{2}$ compact, but must be experimentally verified.

\subsubsection{Metallic Fuels}

The U.S. nuclear industry has proven that metal fuels are safe and effective nuclear fuels. The Experimental Breeder Reactor (EBR)-II reactor was run exclusively on cast metal fuel rods of either 
95\%U-5\%Fs (Fissium) or 90\% U-10\%Zr alloys, totaling tens of thousands of metal fuel rods. In addition to EBR-II, several lead test assemblies utilizing metal fuels were inserted into the Fast Flux test Facility (FFTF) reactor which performed as expected in the reactor. Plutonium production reactors as well as EBR-I have successfully used metallic fuels. The U.S. reactors have traditionally used rod type fuels fabricated using casting, swaging, or extrusion techniques but many of these processes also lend themselves to non-circular cross sections. The main advantage of metallic fuel is its ease of fabrication. One of the other advantages of metal fuels is the ability to produce the fuel to net or near net shape with one casting step. This becomes particularly advantageous if the fuel is a more complex design, because as is shown outside of the nuclear fuel industry, casting to complex shapes is done on a regular basis.

\section{Metallic Fuel feedstock supply}

The Idaho National Laboratory has sufficient existing feedstock to support initial fuel assembly prototyping experimentation and beyond. Sufficient enriched metallic uranium exists across the Department of Energy complex to support fuel fabrication for anticipated unit volumes. The target enrichment of $19.75 \%{ }^{235} \mathrm{U}$ can be achieved through appropriate blending and melting of highly enriched uranium metal with depleted uranium metal to form a homogenized feedstock. This has been accepted as compliant methodology under regulatory review for several programs sponsored by the Department of Energy and NNSA.

\section{Metallic Fuel Casting}

Depending on the surface finish requirements of the final fuel product it is likely that the HPR fuel can be cast to net shape and clad directly with minimal processing. This was the case for all of the EBR-II and metallic FFTF fuels as well as other reactors. The fuel was cast, cut to final length and clad. Because these fuels were sodium bonded, a tight fit against the cladding was not necessary therefore, diametral tolerances were fairly large. However, in a more recent irradiation test, AFC-3A/B, fuels were cast directly to diameter which led to the requisite tight fit into the cladding tubes. This test was run with no fuel failures and is currently undergoing post irradiation examination. This shows the ability to obtain reasonable surface finished and diametric dimensional stability, therefore cladding directly from casting with only minimal processing is feasible, which leads to an economic advantage as well as provides for fewer processes to develop, which decreases technical and schedule risk.

Although in some aspects metallic fuel production for the HPR design may be different than standard metallic fabrication, for example possible batch size and radiological safety concerns, in most aspects it is very similar based on materials properties. This gives the ability to draw from a much larger pool of experience and knowledge. The proposed $90 \%$ uranium 10\% zirconium fuel alloy is a standard fuel alloy that was used for the Integral Fast Reactor project in EBR-II. A large database exists for fuel behavior and past fuel fabrication experience for this alloy. Although the fuel design for the HPR is different form previously cast fuels, the flexibility of the casting process lends itself to more complex geometries. A fabrication route can be developed based on past metallic fuel fabrication activities, and the zirconium and titanium casting in industry. Based on industrial, and DoE complex metal fuel casting experience, fabrication of a metallic fuel meat for the HPR fuel system should be feasible.

\section{Spark Plasma Sintering of metallic fuels}

The INL's Radiological Spark Plasma Sintering (RSPS) Facility located at MFC 784 is capable of producing metallic fuel alloys. SPS is a net shape process that can produce the HPR geometries in metal 
fuel by sintering metal powders at a peak temperature of approximately $1100 \mathrm{C}$ and in under 1 minute of hold time at this temperature. Up to 12 inches per day of metallic fuel in the HPR geometry could be produced in the RSPS. SPS has been developed at INL to be performed in either a metal or graphite die with similar cross sectional geometries as the HPR design. For metal dies and in some cases with graphite dies, a carbon-based lubricant is used. During the sintering process, these form carbide reaction layers that typically extends no more than 10-100 micrometers into the meat of the product. Removal of die lubricants or carbides form the external surface can be performed following SPS via treatment in a hydrogen environment furnace at EFF. To date, the INL's RSPS facility has been used to produce up to 2" across the flats hexagonal metallic fuels with intricate flow channels / annuli (see Figure 11).

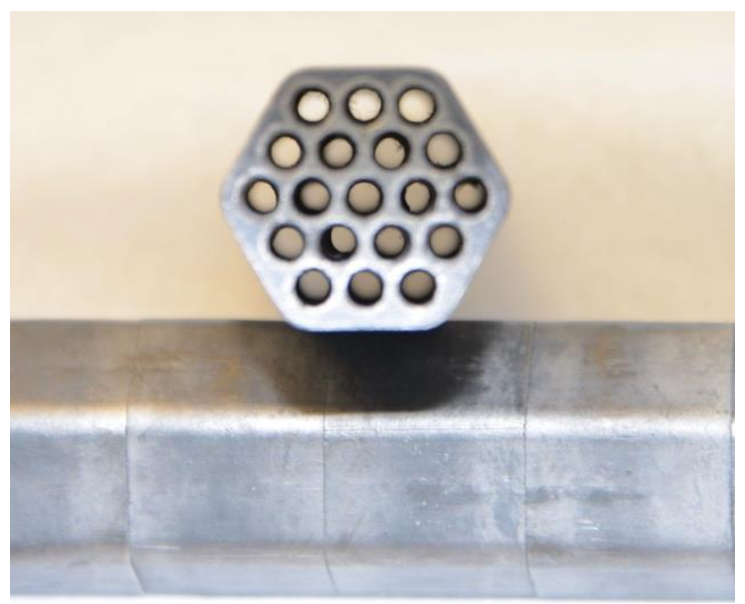

Figure 11: Example of the intricate net-shape processing that is feasible using SPS for the manufacture of metallic fuels [16].

Use of SPS would require the fuel element to be composed of a stack of fuel segments. These segments need not be joined, but bonded to the cladding using sodium metal.

\section{Hot Isostatic Pressing of metallic Fuels}

The INL has a hot isostatic press at MFC FASB that is capable of producing sub-length metal fuel slugs ( 10" length) for the HPR design. HIP has significantly higher production costs over casting, but may result in better uniformity in the finished metal slug in comparison to casting. HIP would also require the post fabrication machining to remove the HIP can from the outside of the part. EDM or mechanical machining can again be used to perform this work.

\subsection{Fuel Element Assembly}

For metallic fuels, sodium $(\mathrm{Na})$ is recommended as a bonding media between the fuel meat and cladding. Alternative metals such as potassium $(\mathrm{K})$ could be considered, but the operational data of $\mathrm{Na}$ as a bonding media provides most confidence. For ceramic fuels, Helium (He) is conventionally and industrially used as a bonding media across gas gaps to the cladding walls. High confidence in helium bonding is provided through commercial operating experience. INL currently has established capability to perform either of the two bonding techniques for fuel-cladding systems. Specialized fixtures will likely be required for either technique in order to accommodate the HPR geometry but could be developed within a 6-month period or less. 
Installation of the heat pipe sub-assembly within a fuel element will be very sensitive to any tolerance stacking on the ID of the internal annulus. Installation would either require that the cladding be brought up to an elevated temperature or for the heat pipe to be installed via hydraulic press-fit. It is recommended that the project seek support from the heat pipe manufacturer to pre-install the heat pipes into the round cladding tubing that can be later brazed or welded into the hexagonal prismatic box section at INL. Such provision could be part of the vendor's assembly process and would minimize damage during final assembly of the elements on-site.

Initial assembly of the element / cladding could include installation of a suitable lower end-plug that is pre-populated with a welded heat pipe / central annulus cladding tube. The lower end-plug would be welded into the hexagonal box section. Either metal fuel slugs or $\mathrm{UO}_{2}$ segments would be stacked into the hexagonal section, passing over the clad heat pipe. For metal fuel, the cladding would be heated with $\mathrm{Na}$ metal inside prior to installation of the metal fuel slugs. Any reflector components (e.g. Be or $\mathrm{BeO}$ ) could be installed into the cladding prior to and after fuel meat installation. Once the fuel meat is loaded into the cladding, a hold down spring would be placed atop the stack and the upper end plug would be joined to the cladding. A weep hole could be provided in the upper end plug to facilitate evacuation and backfill for helium bonding of $\mathrm{UO}_{2}$ fuels.

Stainless Steel (316 SS) has significant heritage in its use for both structural and cladding materials in both metallic fast reactor systems and Light Water Reactor (LWR) systems. The principal driver away from 316 SS to zirconium alloy claddings in LWR power plants was economics in the fuel cycle [17]. $316 \mathrm{~L}$ has a melting point between $1390^{\circ} \mathrm{C}$ and $1440^{\circ} \mathrm{C}$, is corrosion resistant and is resistant to chemical and acid attack. The use with metallic fuels will likely require the provision of a zirconium $(\mathrm{Zr})$ or vanadium (V) liner / barrier to prevent Fuel Cladding Chemical Interaction (FCCI) related failures. Several domestic steel vendors are capable of fabricating 316 SS claddings by either extrusion, drawing or welding. Extrusion will result in the holding of tightest tolerances, estimated to be less than 0.002 " $(0.0508 \mathrm{~mm})$ over the 59 " $(1500 \mathrm{~mm})$ total length. Historically, the EBR-II cladding was held to within $+/-$ 0.001 " on the Outer Diameter (OD) and $+/-0.0005 "$ on the internal diameter (ID). It is likely that similar tolerances will be achievable for the HPR cladding but trial contracts with potential vendors are recommended.

\subsection{Fuel Fabrication Recommendations}

Design A calls for the installation of a heat pipe within the internal diameter of an annulus cladding on the fuel element. It is, however, recommended that the HPR design team consider vendor installation of the heat pipe within the central annulus cladding that can be brazed at the end plugs of each fuel element due to concerns of tolerance stacking during assembly of the heat pipe within a clad annulus.

While metal fuels would allow for immediate assembly of an enriched prototype element, $3 \mathrm{wt} \%$ $\mathrm{Cr}_{2} \mathrm{O}_{3}$ doped $\mathrm{UO}_{2}$ is recommended as the most suitable fuel meat for the HPR fuel element design. This recommendation is made based on the melting point of the $\mathrm{UO}_{2}$ and the manageable swelling and fission product retention behavior of $\mathrm{UO}_{2}$ at the $1 \%$ target burnup. A prototype element at $5 \%$ enriched or depleted uranium is immediately possible with one of several processes. SPS would allow rapid prototypic of doped $\mathrm{UO}_{2}$ fuel meat at MFC 784. Hot isostatic bag pressing could be feasible within a 6-12 month period. This would allow for production rates equivalent to multiple $(\sim 54)$ elements per day. For 
doped $\mathrm{UO}_{2}$ fuel, it is recommended that the fuel meat be in axial segments of no more than 1-inch per segment to minimize thermal stress induced cracking during operation. Helium bonding is the recommended heat transfer mechanism for stainless steel clad doped $\mathrm{UO}_{2}$. Plenum space within each element of no more than 2 inches in length should be sufficient to accommodate fission gas generation. A plenum spring mechanism should be used to hold down the fuel meat stack within the fuel element.

Overall, both single element prototyping, to support irradiation experiments, and large-scale production for core loadings is possible at the INL. Modest infrastructural improvements would be required to achieve large-scale production. Cost estimates could be provided for a specific pathway if required. 


\section{DESIGN B}

Design B is the second of two INL alternative core design concepts for the SPR. The following sections give a preliminary description and analysis of this design concept and its feasibility.

\subsection{Basic Design Features}

A cross-sectional view of the Design B active core is shown in Figure 12. The core is composed of six individual wedge segments, similar to the LANL concept. Each segment is a double-wall tank. The inner tank contains the heat pipes, fuel pins, spacer plates, and liquid metal sodium; the sodium fills the interstitial space between the heat pipes, fuel pins, and spacer plates. This inner tank is a steel structure that is sealed. The outer tank is also a steel structure, but is separate from the inner tank, and engineered to provide added insurance for the containment of the sodium in the inner tank. The outer tank is also sealed with side, top, and bottom steel walls, where the top and bottom walls also act as the top and bottom neutron reflectors. Partitioning of the active core into six double-tank segments further ensures minimal loss of the sodium in the event of a tank breach. The ex-core structures outside the active core: alumina side reflector, control drums, emergency shutdown rod(s), core barrel, and radiation shield will be similar to the LANL and Design A concepts. There are, however, a few minor differences in radial dimensions. Design B reactor core is vertically oriented like Design A.

Preliminary details of the inner and outer tanks are shown in Figures 13, 14, and 15. The inner tank is composed of a top and bottom steel plate and four steel side plates. The inner tank could be a seamless structure with a welded top plate to prevent the possibility of sodium drainage due to gravity. The fuel pins and heat pipes will be held in position by lower and upper grid plates. The heat pipes will penetrate both the upper plate of the inner tank and the upper reflector of the outer tank, requiring seal welds at each wall penetration.

Sodium is used to thermally bond the fuel pins (heat source) to the heat pipes (heat sink) in order to facilitate heat transfer. Some convective circulation of the sodium may occur inside the inner tank primarily between the spacer plates, but the sodium is not intended to function as a circulating coolant. The volume of sodium in the inner tank occupies only $7 \%$ of the total inner tank volume, or approximately 12.4 liters per core segment. For the six core segments, the total sodium volume is only 74.4 liters $(59.3 \mathrm{kgs})$.

Figure 13 shows a small cluster of fuel pins (yellow) surrounding a heat pipe (red) with four spacer plates. There are 204 heat pipes and 352 fuel pins in each inner tank or core segment; the same as the LANL concept. The key difference between Design B and the LANL concept again is the removal of the LANL steel monolith core structure and its replacement with individual heat pipes and fuel pins in a sodium bath. The heat pipes are now a $1 \mathrm{~mm}$ thick stainless steel tube. The fuel pins are composed of cylindrical $\mathrm{UO}_{2}$ fuel pellets with a dedicated $0.3 \mathrm{~mm}$ thick stainless steel clad. The interstitial space outside the heat pipes and fuel pins is filled with liquid metal sodium. The sodium not only thermally bonds the fuel pins to the heat pipes, but also eliminates the thermal stress problem associated with the stainless steel monolith structure. Elimination of the steel monolith thermal stress problem could be a big selling point for Design B.

The $\mathrm{UO}_{2}$ fuel pellets have a slightly larger $1.492 \mathrm{~cm}$ diameter than the $1.412 \mathrm{~cm}$ diameter pellets in the LANL design. The slightly larger diameter adds fuel and reactivity to the core to compensate for a 
slight increase in lattice pitch. The lattice pitch in Design B is $1.8 \mathrm{~cm}$ versus $1.6 \mathrm{~cm}$ in the LANL concept. The Design B heat pipe is essentially the same as the Design A heat pipe. Both have an inner diameter of $1.575 \mathrm{~cm}$ and a $1.0 \mathrm{~mm}$ stainless steel wall thickness. The LANL concept has the same $1.575 \mathrm{~cm}$ inner diameter, but its containment wall (evaporator section) is the steel monolith structure, which varies in thickness around the circumference (1.0 mm minimum thickness). The slightly larger lattice pitch (1.8 $\mathrm{cm}$ ) is needed to accommodate the fuel pin clad, heat pipe wall, and the thin web and gap associated with the spacer plates in Design B.

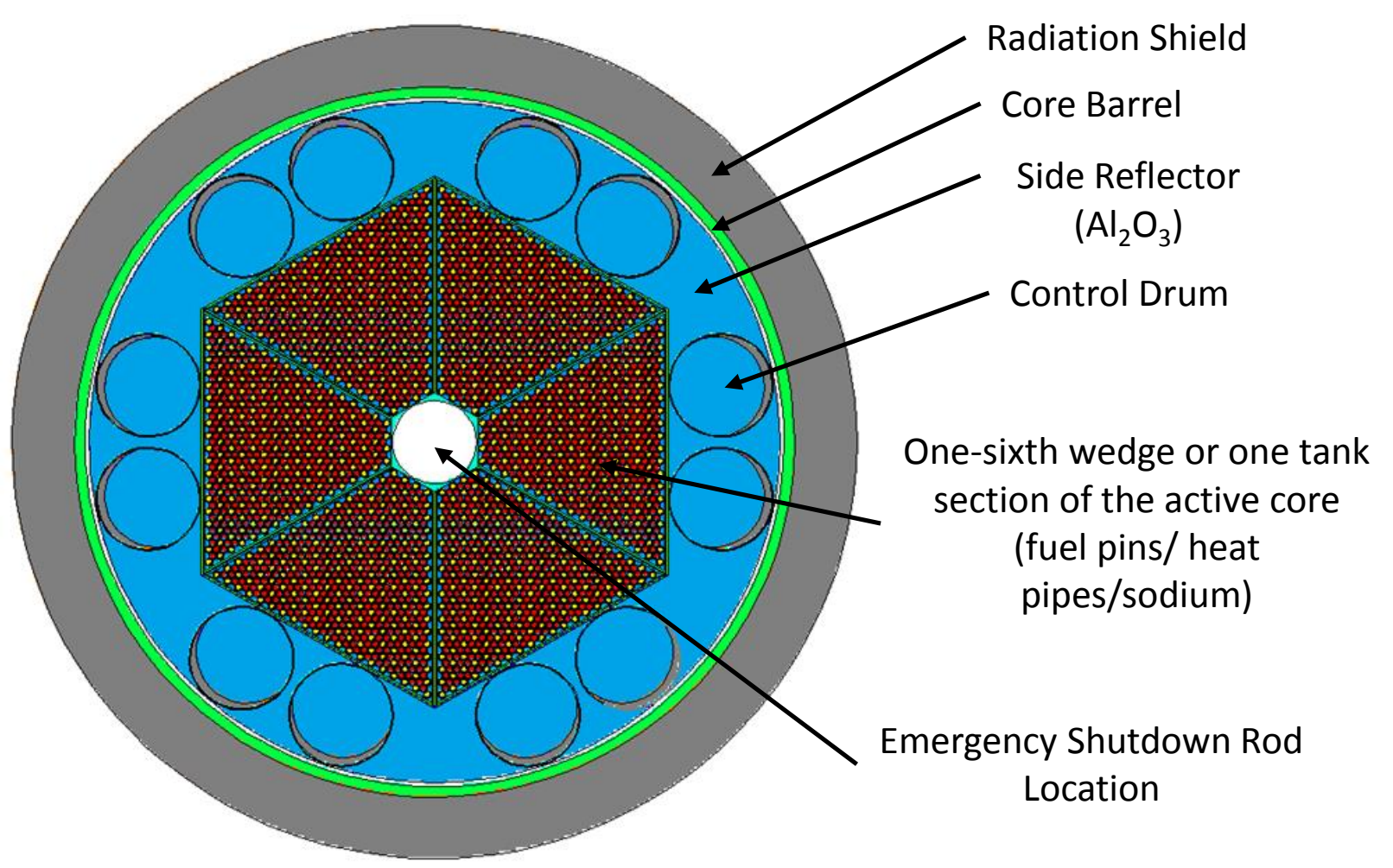

Figure 12. Design B cross-sectional view of the active core. 


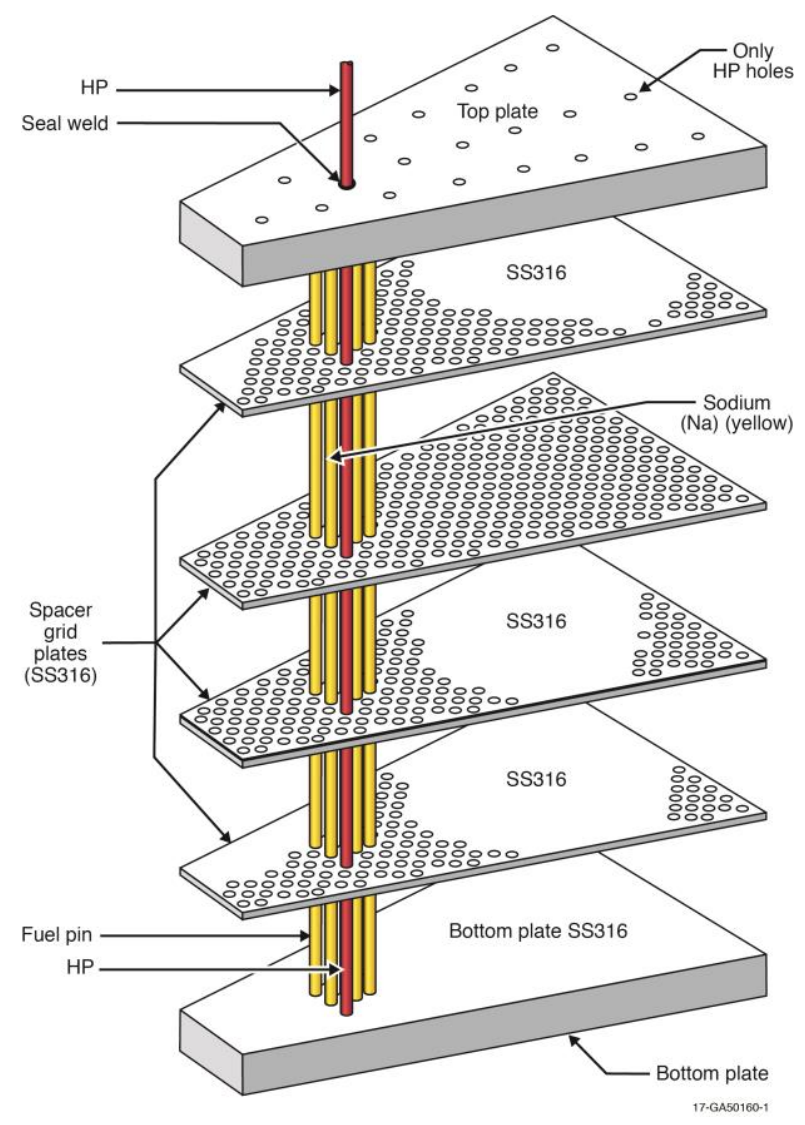

Figure 13. Design B fuel pin and heat pipe arrays inside the inner tank.

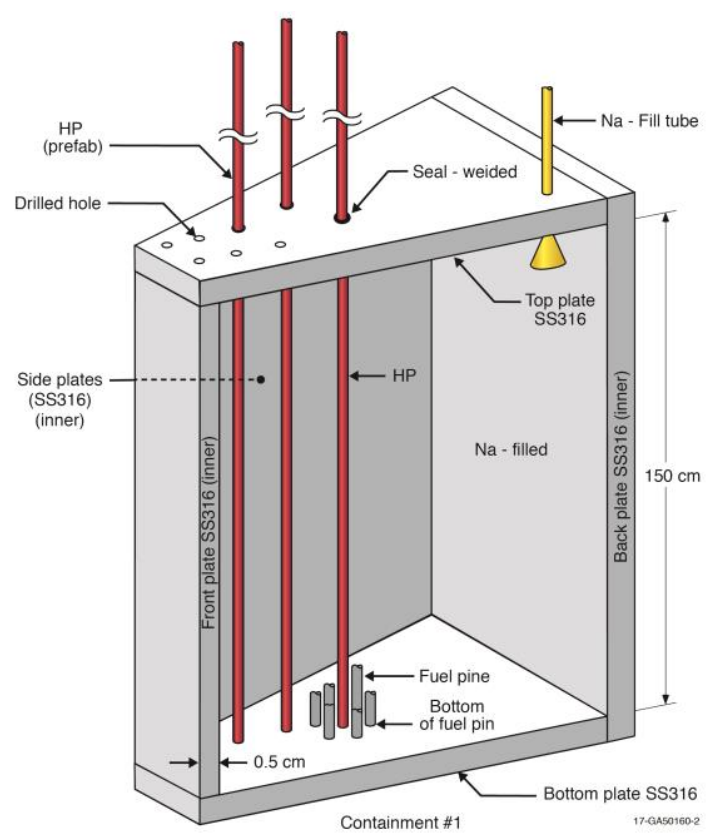

Figure 14. Design B inner tank which contains the fuel pins, heat pipes, and liquid metal sodium. 


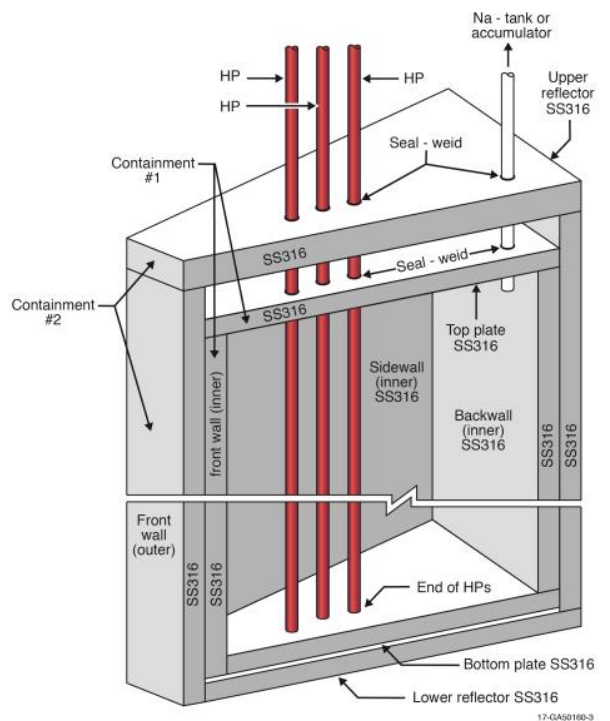

Figure 15. Design B outer tank composed of the top and bottom reflectors and sidewall steel plates.

\subsection{Neutronic Analysis}

The neutronic characteristics of Design B are similar to both Design A and the LANL concept, as intended. The main difference between Design B and the LANL concept, as mentioned previously, is the replacement of the steel monolith with a liquid metal sodium bath. The sodium provides a strong thermal bond between the fuel pins and heat pipes and eliminates the thermal stresses associated with the steel monolith. Replacement of the parasitic steel associated with the monolith structure with liquid metal sodium gives a significant boost in reactivity to the Design B core. Steel tends to absorb neutrons better than the sodium. The boost in reactivity, however, is needed to compensate for the slightly larger pitch in Design B. The increase in pitch increases neutron leakage and therefore, decreases core reactivity.

The LANL design is highly optimized neutronically, and any increase in the core size, even a small increase in pitch, as is the case here for Design B, will drop the core reactivity precipitously. The positive reactivity gain by the replacement of the parasitic steel monolith structure with sodium, however, just balances the negative reactivity loss due to the increase in pitch. Design B beginning-of-life core excess reactivity is $\$ 3.28$, or just slightly higher than the LANL concept at $\$ 2.97$.

The use of liquid sodium in the Design B concept raised concerns by the Industrial Advisory Board due to a negative perception of sodium in general. The board also raised potential concerns related to the shipment of sodium; concerns associated with regulations that might be imposed by Nuclear Regulatory Commission (NRC) and Department of Transportation for the shipment and transport of sodium. The negative perception of sodium stems from its potential to ignite and burn in contact with water. Water 
being ubiquitous in all natural environments leads to a variety of imaginable accident scenarios. However, sodium has been successfully used in fast reactors around the world without incident, and the handling, usage, and physical properties are well-known. For Design B, the total amount of sodium in the core is less than 75 liters-not a large amount. Combined with double-tank encapsulation in each core segment, the probability of sodium-leakage out of, or water-ingress into, any one of the six core segments is very small. Another positive attribute for Design B is the negative sodium void coefficient of reactivity. The void coefficient is calculated to be negative, hence, any sodium-leakage (drainage) out of a core segment will simply shut down the reactor.

The greatest concern with the use of sodium is the loss of sodium after some period of reactor operation. Loss of sodium, or the thermal heat transfer medium between fuel pin and heat pipe, could potentially allow the fuel pins to overheat due to the decay heat generated in the fuel pins after reactor shutdown. To alleviate this potential problem, additional conduction heat transfer pathways could be designed into the inner tank structure to address the decay heat issue (more spacer plates, thicker spacer plates, bonding of spacer plates to fuel pins, axial heat sinks in the top and bottom grid plates, core segment radial conduction paths). The loss of sodium would only occur if both tank walls were breached, a low probability event, especially for secure civilian locations or subterranean concrete vault structures in less secure locations.

Since there are few other differences in the Design B and LANL core designs, most other neutronic characteristics are also similar. Examples include: negative feedback coefficients of reactivity, control drum worth, emergency shutdown worth, neutron spectra, and core burnups. Therefore, Design B should have operational characteristics very similar to both Design A and the LANL concept under steady-state and transient conditions. Radiation streaming is still a concern as is accidental flooding of the heat pipes.

A viable alternative to $\mathrm{UO}_{2}$ fuel is the metallic fuel form $\mathrm{U}-10 \mathrm{Zr}$. This fuel form is $90 \mathrm{wt} \%$ uranium metal and $10 \mathrm{wt} \%$ zirconium metal. The theoretical density of $\mathrm{U}-10 \mathrm{Zr}$ is higher than $\mathrm{UO}_{2}(16.0$ versus $\left.10.96 \mathrm{~g} / \mathrm{cm}^{3}\right)$ as is the uranium density $\left(14.40\right.$ versus $\left.9.66 \mathrm{~g} / \mathrm{cm}^{3}\right)$. The higher uranium density allows for a nearly $50 \%$ increase in uranium loading in the core which can translate into either: (1) a smaller fuel pin diameter, which in turn allows for a smaller lattice pitch and a more compact core, or (2) a lower fuel enrichment. For example, the fuel enrichment could, as is the same for Design A, be lowered from 19.75 to less than $15 \mathrm{wt} \% \mathrm{U}-235$. The metallic fuel form has the added advantage of higher thermal conductivity, so fuel temperatures will be lower relative to $\mathrm{UO}_{2}$.

Relative to oxide ceramic fuels, metallic fuel in high-burnup reactor cores typically exhibit swelling, fission gas release, fuel redistribution, lower melting point, and fuel-clad interactions. However, since the SPR core burnup, even after 5 years of operation, is so small, the effects of fuel swelling due to fission gas production would be minimal. Deliberate introduction of porosity into the metal fuel to counter these effects would probably be unnecessary, and therefore, near-full density fuel could be used in Designs B and A. Introduction of a metallic fuel form, such as $\mathrm{U}-10 \mathrm{Zr}$, into the Design $\mathrm{B}$ core by replacing the $\mathrm{UO}_{2}$ ceramic fuel form appears to have some very positive advantages. Further analysis is required to exploit this potential.

For a more complete look at all the preliminary neutronic analysis of Design B, see Appendix B. Appendix B describes the computer code software, models, and calculated results, plus provides comparisons to the LANL concept. 


\subsection{Thermal Analysis}

A preliminary thermal analysis has been performed for Design B under normal steady-state operating conditions. Table 5 gives peak temperatures calculated for the major in-core components in an inner sodium tank. For comparison purposes, corresponding peak temperatures for the LANL concept [6] are provided in the table. For the LANL concept, two sets of peak temperatures are presented, one for temperatures calculated assuming an isothermal heat pipe wall temperature of $677^{\circ} \mathrm{C}$ per reference [5], and a second set of temperatures (in parentheses) adjusted to an isothermal heat pipe wall temperature of $712.5^{\circ} \mathrm{C}$. The higher isothermal temperature of the heat pipe wall $\left(712.5^{\circ} \mathrm{C}\right)$ is based on more knowledgeable calculations performed after the publication of reference [6].

The peak $\mathrm{UO}_{2}$ fuel temperatures between Design B and the LANL concept are similar in magnitude (around $780^{\circ} \mathrm{C}$ ). Relative to commercial U.S. light water reactor fuel temperatures, the SPR fuel temperatures are low, as mentioned above in Design A. This should allow the SPR $\mathrm{UO}_{2}$ fuel matrix to retain much of the fission gas and minimize gas-plenum volume allocation requirements. Thermal stress in the fuel and clad have not yet been calculated, but are expected to be minor concerns. The liquid metal sodium temperature ranges from approximately $713-730^{\circ} \mathrm{C}$, or well below the $883^{\circ} \mathrm{C}$ boiling point of sodium.

Table 5. Peak temperatures calculated for Design B and the LANL concept.

\begin{tabular}{|c|c|c|c|}
\hline \multicolumn{2}{|c|}{ Design B } & \multicolumn{2}{c|}{ LANL Concept } \\
\hline $\mathrm{UO}_{2}$ fuel & $777^{\circ} \mathrm{C}$ & $\mathrm{UO}_{2}$ fuel & $753^{\circ} \mathrm{C}\left(789^{\circ} \mathrm{C}\right)$ \\
\hline $\mathrm{Clad}$ & $740^{\circ} \mathrm{C}$ & Monolith & $696^{\circ} \mathrm{C}\left(731.5^{\circ} \mathrm{C}\right)$ \\
\hline $\begin{array}{c}\text { Heat pipe wall } \\
\text { (isothermal) }\end{array}$ & $712.5^{\circ} \mathrm{C}$ & $\begin{array}{c}\text { Heat pipe wall } \\
\text { (isothermal) }\end{array}$ & $677^{\circ} \mathrm{C}\left(712.5^{\circ} \mathrm{C}\right)$ \\
\hline Sodium & $713-730^{\circ} \mathrm{C}$ & ---- & --- \\
\hline
\end{tabular}

Appendix D provides more detail on the preliminary Design B thermal models, codes, input data, and analysis results.

\subsection{Heat Pipes}

The same heat pipe design, as in Design A, is also proposed for Design B. The nominal core design for Design B calls for 1,224 heat pipes, like the LANL concept, but with the heat pipes oriented in the vertical direction. At $5 \mathrm{MWt}$, each Design B pipe is expected to lift on average $4.09 \mathrm{~kW}$, similar to the LANL concept. The heat pipe discussion for Design A above, and Appendix E heat pipe analysis applies here to Design B as well. 


\subsection{Manufacturability}

The $\mathrm{UO}_{2}$ fuel form and cylindrical fuel pellets are expected to be comparable to commercial $\mathrm{UO}_{2}$ fuel. The main difference will be the higher SPR enrichment. Fuel pellet diameter and length may also exhibit slight differences. The Type 316 stainless steel fuel clad (circular tube) should be readily manufacturable at the required dimensions and specification using the U.S. steel-product vendors. 


\section{CONCLUSION}

Two new alternative active core designs (Design A and Design B) are proposed and preliminarily evaluated for the Special Purpose Reactor concept. The two new core designs essentially replace the stainless steel monolithic core structure in the Los Alamos National Laboratory Mega-Power with more standard fuel element designs and core structures. The two new core designs will still retain the basic Mega-Power operating characteristics including: total core power, $\mathrm{UO}_{2}$ fuel, passive heat pipe-cooling, fast-spectrum, low burnup, and all ex-core components including the same proposed power conversion unit. Based on the preliminary supporting analyses for Design A and Design B herein, both are deemed viable active core concepts for the Special Purpose Reactor, although Design A is currently preferred over Design B.

An important goal of both Design A and Design B was to ensure that the active core components could be readily manufactured by U.S. commercial vendors using existing fabrication technologies. This appears to be the case after a survey of U.S. tube, plate, fuel, and heat pipe vendors. The SPR Industrial Advisory Board composed of leading U.S. manufacturing experts also offered support and acknowledgement that these two designs could be readily manufactured. Because Design A and Design B intend to use code-qualified materials and components with high technology readiness levels, both design concepts could be expected to support aggressive deployment schedules.

To enhance the Special Purpose Reactor's defense-in-depth, both Design A and Design B have dedicated cladding around the $\mathrm{UO}_{2}$ fuel in addition to other encapsulating core structures. The fuel elements in Design A and the fuel pins in Design B along with the heat pipes in both are all designed to be fabricated individually. As individual standalone items, the fuel elements, pins, and heat pipes can all be manufactured using dedicated fabrication lines and processes. Each element, pin, and pipe will be identical to the next. Plus, each can be assembly, inspected, tested, loaded, sealed, and qualified to meet their respective construction specifications. This will ensure and greatly enhance component reliability and performance for the reactor over its lifetime. This may not be the case with the steel monolith core structure. These advantages in Designs A and B could be of significant benefit when it comes to NRC licensing of the reactor.

Calculated thermal stresses in the LANL stainless steel monolithic core structure may suffer very high stress levels, possibly beyond ASME allowable levels for Type 316 stainless steel at the normal operating temperature of $700^{\circ} \mathrm{C}$. Design A and Design B both have stainless steel in their cores as well, but in the form of non-loading bearing, non-pressure boundary, vertically-orientated claddings. Under the Special Purpose Reactor high-temperature operating conditions, these cladding structures should experience reduced stress levels. Stresses in the Design A fuel elements are preliminarily calculated to be approximately 8 times less than those of the monolith, well below the ASME limits. For the LANL monolith structure, thermal stress levels could potentially be reduced with a reduction in core power or an increase in monolith webbing thickness. A reduction in power translates into a comparable reduction in electrical output, and thicker webbing translates into a larger core footprint. Power reductions are not expected for Design A or Design B.

The use of $\mathrm{UO}_{2}$ is currently the preferred fuel form for the SPR concepts, because of its high technology readiness level, which can in turn better support an aggressive reactor deployment schedule. However, the use of a higher density metallic fuel form, such U-10Zr, appears to have significant and 
interesting design potential for both Design A and Design B. For Design A, a significant reduction in enrichment could be realized by dropping from $19.75 \mathrm{wt} \%$ down to less than $15 \mathrm{wt} \% \mathrm{U}-235$, while maintaining the same core excess reactivity and burnup specifications. For Design B, the fuel pellet diameter or enrichment, or both could be reduced. An enrichment reduction to less than $15 \mathrm{wt} \% \mathrm{U}-235$ could also be realized for Design B. Low burnup cores, like the SPR cores here would be well suited for a $\mathrm{U}-10 \mathrm{Zr}$ fuel form.

Finally, one very exploitable feature of the Special Purpose Reactor is its low power level (5 MWt). The relatively low power will allow for the construction of a full-scale prototypical reactor core or engineering demonstration unit that uses electrical heaters in place of the nuclear fuel to mimic the operational functions of the complete reactor system and its sub-systems. The instrumented engineering demonstration unit would provide the necessary means to simulate both normal and off-normal operation of the reactor system and provide the temperature, stress, strain, heat transfer, heat pipe function, and power conversion unit function data to assess the system performance. The measured data would also provide the necessary verification of the computer models, computer codes, calculated design analyses, operating limits, thermal margins, and the final validation of the expected system performance prior to the deployment and licensing of the first-of-a-kind Special Purpose Reactor. In addition, instrumented engineering tests for individual heat pipes and power conversion unit could also be performed prior to integration into the reactor engineering demonstration unit. 


\section{REFERENCES}

[1] "Task Force on Energy Systems for Forward/Remote Operating Bases," Final Report, U.S. Department of Defense, Defense Science Board, August 1, 2016.

[2] MINTZ, S., "Military's future could rely on tiny nuclear reactors", E\&E News, www.eenews.net, July $10,2017$.

[3] RAO, D.V., et. al., "Special Purpose Reactors for Powering DOD Operations," LA-UR-13-25412, Los Alamos National Laboratory.

[4] MCCLURE, P.C., et. al., "Safe Affordable Fission Energy for Mobile Army Remotes Site Power (2MWe, Mobile Heat Pipe Cooled Fast Reactor)," Los Alamos National Laboratory, private information, February 2017.

[5] MCCLURE, P.C., et. al., "Design of Megawatt Power Level Heat Pipe Reactors," LA-UR-15-28840, Los Alamos National Laboratory, November 12, 2015.

[6] STERBENTZ, J. W., et. al., "Special Purpose Nuclear Reactor (5 MW) for Reliable Power at Remote Sites Assessment Report,” INL/EXT-16-40741, Rev. 1, April 2017.

[7] EL-GENK, M.S. and J.M.P. Tournier, "Uses of Liquid-Metal and Water Heat Pipes in Space Reactor Power Systems", Frontiers in Heat Pipes (FHP), 2, 013002 (2011).

[8] DUDERSTADT, J.J., and L.J. Hamilton, "Nuclear Reactor Analysis”, John Wiley \& Sons, copyright 1976. ISBN 0-471-22363-8.

[9] WALTAR, A.E. and A.B. Reynolds, "Fast Breeder Reactors", Pergamon Press, 1981. ISBN 0-08025983-9.

[10] WOLOSHUN, K.A. et. al., "HTPIPE: A Steady-State Heat Pipe Analysis Program”, LA-11324-M, Los Alamos Scientific Laboratory, 1988.

[11] O’BRIEN, R. C., et. al., "Fuel Fabrication Pathways for Compact Heat Pipe Special Purpose Reactors," INL/INT-17-41672, Rev. 0, June 2017.

[12] GUPTA, C. K. and H. Singh, Uranium Resource Processing, Springer-Verlag, Berlin, Germany, 2003, chapter 8 .

[13] LACHER, J. R.; Salzman, J. D.; Park, J. D., Ind. \& Eng. Chem. 1961, 53(4), 282-284.

[14] DELAFOY, C. and I. Arimescu "Developments in fuel design and manufacturing in order to enhance the PCI performance of AREVA NP'S fuel", proceedings of 2016 PCI workshop, Lucca, Italy.

[15] WAECKEL, N., "General overview of the French effort to address the SCC-PCI issue", proceedings of 2016 PCI workshop, Lucca, Italy. 
[16] O’BRIEN, R.C., “Aerojet Rocketdyne Cermet Fuels Research: Summary \& Status”, Proceedings of 2015 Nuclear and Emerging Technologies for Space conference, Albuquerque, New Mexico, February $25,2015$.

[17] ROBERTS, Adrian T., "Structural Materials In Nuclear Power", Springer US, 2013, ISBN 1468471953, 9781468471953. 


\section{Appendix A}

\section{Neutronics Analysis -- Design A}

This appendix presents details of a preliminary neutronic analysis for Design A. Included in this appendix are descriptions of the computer codes, computer models, and assumptions used to perform the parametric studies in order to evaluate Design A reactor thermal sensitivities and characteristics to help evaluate and support the overall Design A reactor system. 


\section{Design A}

\section{INTRODUCTION}

INL has previously performed an independent assessment of the Special Purpose Nuclear Reactor design proposed by Los Alamos National Laboratory [1]. This was completed in April of 2017, and as the report notes, there are several potential reactor design concerns. The original design proposed by LANL consists of a stainless steel (SS) monolith structure with individual fuel and heat pipe channels arranged in a triangular pitch [2]. The core is separated into 6 symmetrical $60^{\circ}$ sectors with the monolith structure also serving as the cladding. Due to monolith fabrication concerns and the lack of a defense in depth approach to fission product release, two alternative design concepts, referred to as Designs A and B, have been proposed by INL to alleviate or bypass these issues. These two designs proposed by INL sought to have an independent cladding for each fuel and heat pipe element and avoid construction of the monolith as proposed $^{1}$. From a neutronic standpoint, it was very important that these design alternatives aim to: 1) keep a roughly equivalent core footprint/size as the LANL design 2) use the same material compositions and enrichments 3) maintain similar margins of reactivity control and 4) operate continuously and safely for a minimum of five years. The continuous energy Monte Carlo radiation-transport code MCNP6.1 with the ENDF-7.0 nuclear data was used for all calculations unless stated otherwise [3].

The Design A core analysis is discussed first, beginning with the core layout, geometry specifications, and materials used. Section 2 examines the primary and secondary core reactivity effects as well as a worst case scenario by which the cores flood with water. The thermal parameters, burnup evaluation, decay heat analysis, and potential dose problems are presented in Section 3. Finally, parametric and sensitivity studies related to the heat pipe diameters and different cladding thicknesses are presented in Section 4. The Design B core analysis is then discussed in a similar fashion. Any significant discrepancies between these two designs and the LANL reference case are noted.

\section{Core Description}

The defining feature of Design A is that each heat pipe is inserted inside an annular hexagonal fuel element (both independently clad). As seen in Figure 1, each unit cell consists of the heat pipe working fluid surrounded by stainless steel (SS) clad, gap, inner fuel SS clad, gap, hexagonal fuel meat, gap, and finally the outer fuel SS clad. The active core, shown in Figure 2, consists of 1134 of these unit cells arranged in a triangular pitch to form a larger hexagon roughly $1 \mathrm{~m}$ across flats. Each unit cell has an axial $\mathrm{BeO}$ upper and lower reflector with a fission gas plenum at the bottom of each element. All relevant dimensions are given in Table 1.

The active core is surrounded by a radial alumina reflector containing 12 rotatable control drums. Each alumina control drum contains a $90 \%$ (B-10) enriched $\mathrm{B}_{4} \mathrm{C}$ arc with a maximum thickness of $2 \mathrm{~cm}$. The reflector is surrounded by $5.08 \mathrm{~cm}$ ( 2 inch) thick SS core barrel and a $15.24 \mathrm{~cm}\left(6\right.$ inch) thick $\mathrm{B}_{4} \mathrm{C}$ neutron shield. The center of the core contains a voided area to be used for insertion of an emergency shutdown rod if needed. A radial full core schematic is shown in Figure 3. All designs are characterized by a fast neutron spectrum, utilize $19.75 \% \mathrm{LEU} \mathrm{UO}_{2}$ fuel and are designed to operate at $5 \mathrm{MW}(\mathrm{t})$ for 5 years.

Table 2 lists the number densities for all materials used in the calculations. The number densities of liquid potassium and potassium vapor in the heat pipes assume a temperature of $625^{\circ} \mathrm{C}(925 \mathrm{~K})$ and $0.101 \mathrm{MPa}$

\footnotetext{
${ }^{1}$ A monolith structure could very easily offer many advantages. However, based off the information obtained by INL the structure would have to be larger than proposed, thus increasing the lattice pitch and overall reactor footprint.
} 
[4]. The number densities for the liquid metal sodium in Design B assumes a temperature of $625^{\circ} \mathrm{C}(925$ K) [5].

Table 1. Design A core dimensions and parameters.

\begin{tabular}{|l|l|l|l|}
\hline \multicolumn{4}{|c|}{ Design A Unit Cell and Lattice Dimensions / Core Parameters } \\
\hline K vapor radius $(\mathrm{cm})$ & 0.71 & Pitch $(\mathrm{cm})$ & 2.786 \\
\hline K liquid radius $(\mathrm{cm})$ & 0.7875 & Fuel area $\left(\mathrm{cm}^{\wedge} 2\right)$ & 2.90 \\
\hline HP SS clad radius $(\mathrm{cm})$ & 0.8875 & Fuel pin height $(\mathrm{cm})$ & 150 \\
\hline Gap radius $(\mathrm{cm})$ & 0.8939 & Fuel pin volume $(\mathrm{cc})$ & 435 \\
\hline Inner fuel SS clad radius (cm) & 0.9339 & Inner core hex center-to-flat $(\mathrm{cm})$ & 9.551 \\
\hline Gap radius $(\mathrm{cm})$ & 0.9403 & Inner core circle radius $(\mathrm{cm})$ & 9.05 \\
\hline Fuel hex center-to-flat $(\mathrm{cm})$ & 1.2802 & Outer core hex center-to-flat $(\mathrm{cm})$ & 49.70 \\
\hline Gap hex center-to-flat $(\mathrm{cm})$ & 1.2866 & $\mathrm{UO}_{2}(\mathrm{~kg})$ & 5190 \\
\hline Outer fuel SS clad center-to-flat $(\mathrm{cm})$ & 1.3866 & $\mathrm{U}(\mathrm{kg})$ & 4573 \\
\hline Outer unit cell gap center-to-flat $(\mathrm{cm})$ & 1.393 & ${ }^{235} \mathrm{U}(\mathrm{kg})$ & 903 \\
\hline Axial BeO reflectors $(\mathrm{cm})$ & 15.0 & Annular shutdown rod inner/outer radius $(\mathrm{cm})$ & $6.85 / 8.85$ \\
\hline Lower fission gas plenum $(\mathrm{cm})$ & 20.0 & Solid shutdown rod radius $(\mathrm{cm})$ & 5.6 \\
\hline
\end{tabular}

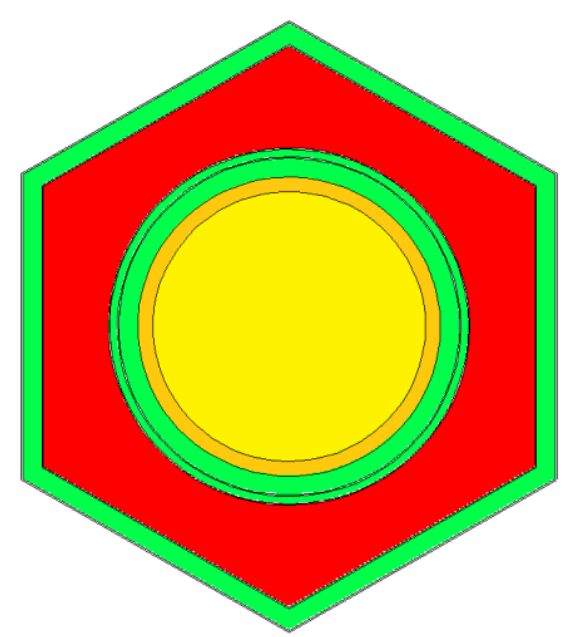

Figure 1. Unit cell structure for Design A. The SS (green) clad heat pipe (yellow) rests inside the hexagonal fuel element (red). The orange ring represents the potassium liquid layer in the heat pipe. 


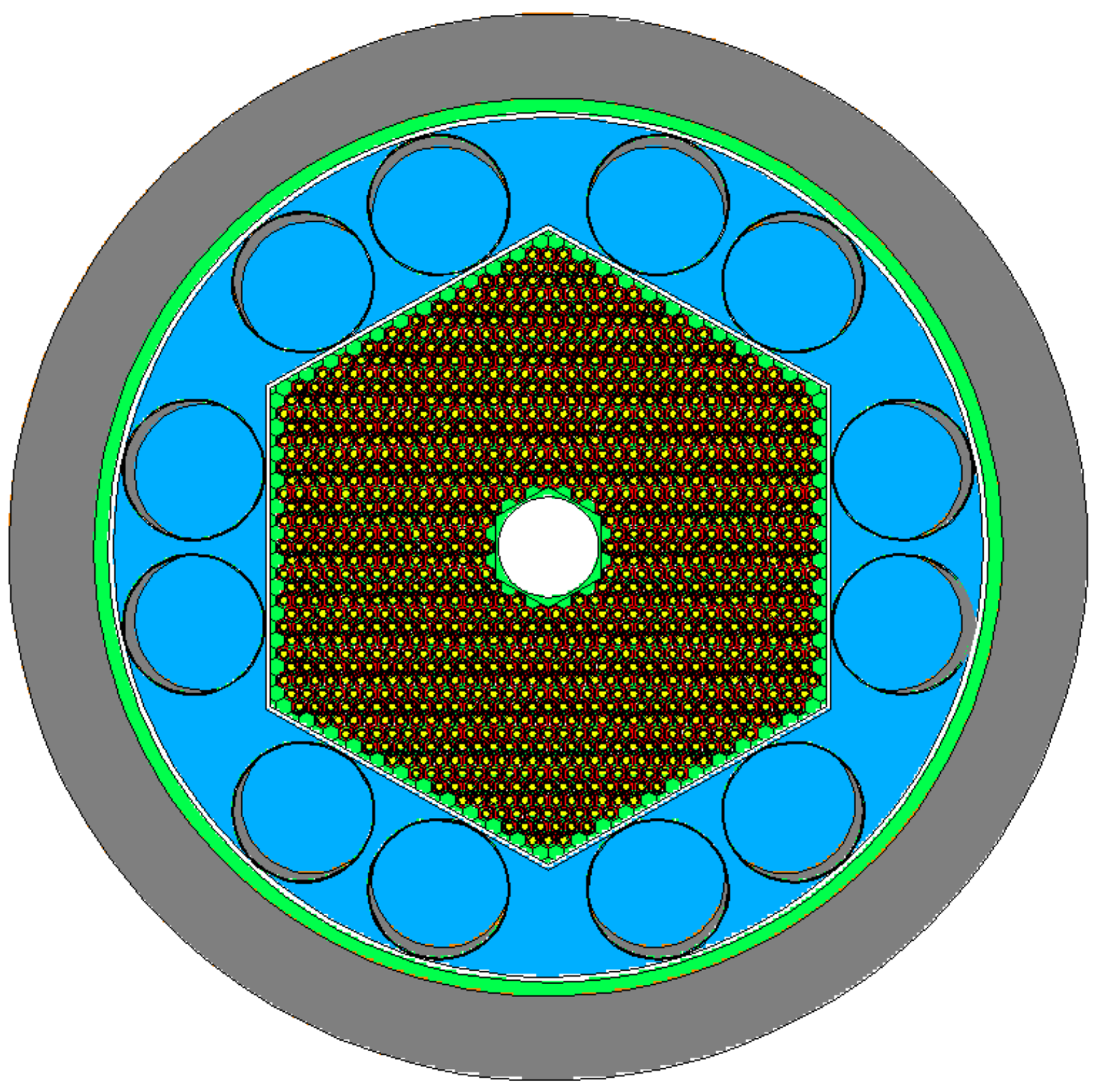

Figure 2. Cross section view of the active core lattice arrangement for Design A surrounded by an alumina (blue) reflector with 12 rotatable control drums. The core consists of 1134 hexagonal fuel/heat pipe unit cells. 


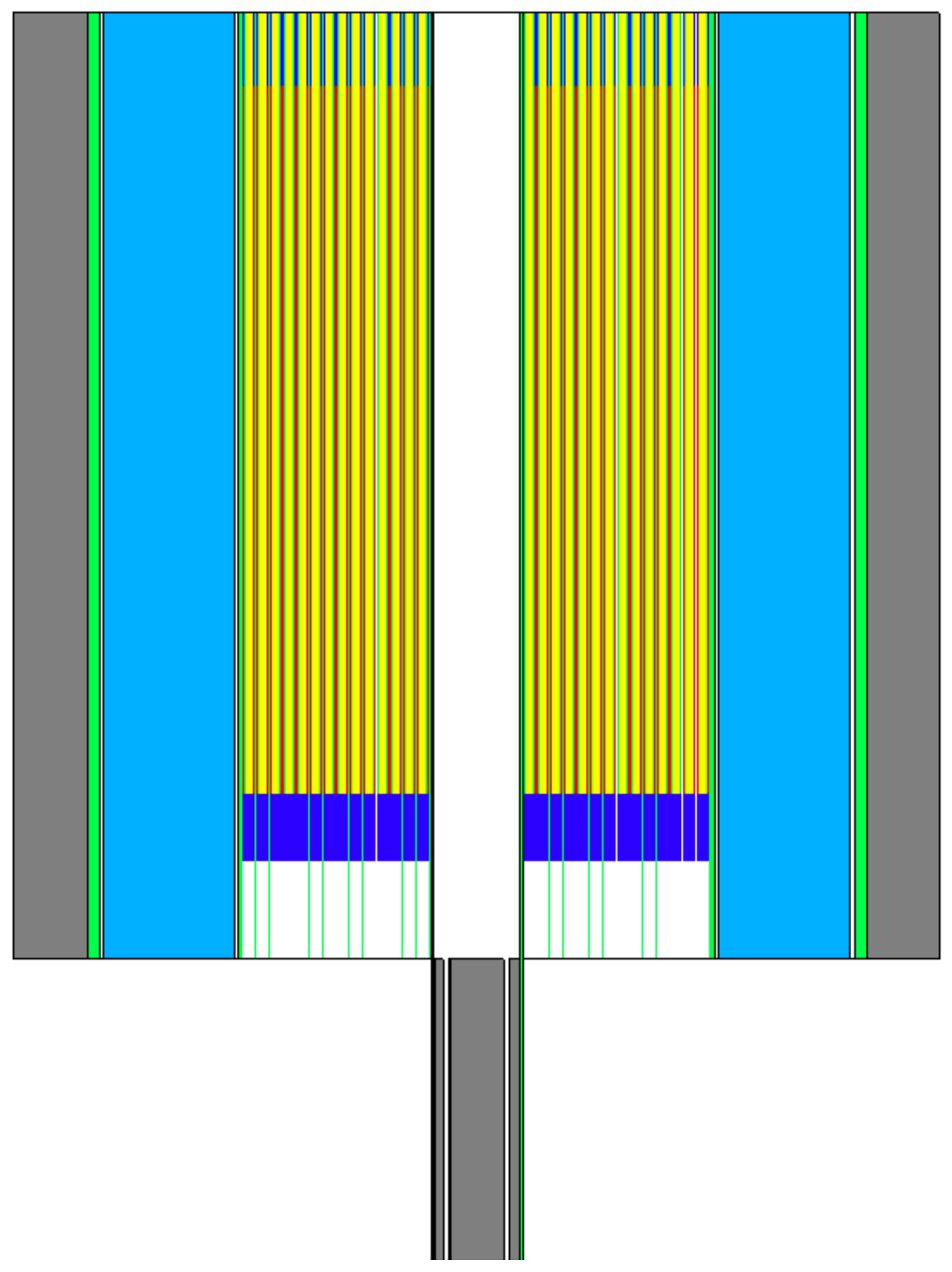

Figure 3. Axial view of the full core for Design A. The core has both an annular and solid $\mathrm{B}_{4} \mathrm{C}$ emergency shutdown rod that can be inserted from the bottom. 
Table 2. Material number densities used in the calculations.

\begin{tabular}{|c|c|c|c|}
\hline Material & $\begin{array}{l}\text { Number Density } \\
\text { (atom/barn-cm) }\end{array}$ & Material & $\begin{array}{l}\text { Number Density } \\
\text { (atom/barn-cm) }\end{array}$ \\
\hline UO $_{2}$ Total & $7.5046 \mathrm{E}-02$ & SS-316 Total & $8.5960 \mathrm{E}-02$ \\
\hline U-234 & $3.5787 \mathrm{E}-05$ & $\mathrm{Fe}-54$ & $3.2697 \mathrm{E}-03$ \\
\hline U-235 & $4.6918 \mathrm{E}-03$ & Fe-56 & $5.1327 \mathrm{E}-02$ \\
\hline U-238 & $1.8788 \mathrm{E}-02$ & $\mathrm{Fe}-57$ & $1.1854 \mathrm{E}-03$ \\
\hline $\mathrm{O}$ & $4.7031 \mathrm{E}-02$ & $\mathrm{Fe}-58$ & $1.5775 \mathrm{E}-04$ \\
\hline K Total (liquid) & $1.0579 \mathrm{E}-02$ & $\mathrm{Cr}-50$ & $6.7739 \mathrm{E}-04$ \\
\hline K-39 & $9.8657 \mathrm{E}-03$ & Cr-52 & 1.3063E-02 \\
\hline K-40 & $1.2377 \mathrm{E}-06$ & $\mathrm{Cr}-53$ & 1.4812E-03 \\
\hline $\mathrm{K}-41$ & 7.1198E-04 & Cr-54 & $3.6870 \mathrm{E}-04$ \\
\hline K Total (vapor) & 5.8109E-06 & $\mathrm{Ni}-58$ & $6.6375 \mathrm{E}-03$ \\
\hline K-39 & $5.4191 \mathrm{E}-06$ & $\mathrm{Ni}-60$ & $2.5568 \mathrm{E}-03$ \\
\hline $\mathrm{K}-40$ & 6.79eeE-10 & $\mathrm{Ni}-61$ & $1.1114 \mathrm{E}-04$ \\
\hline K-41 & $3.9109 \mathrm{E}-07$ & $\mathrm{Ni}-62$ & 3.5436E-04 \\
\hline BeO Total & $1.3772 \mathrm{E}-01$ & $\mathrm{Ni}-64$ & $9.0246 \mathrm{E}-05$ \\
\hline $\mathrm{Be}$ & $6.8860 \mathrm{E}-02$ & Мo-92 & $1.8402 \mathrm{E}-04$ \\
\hline $\mathrm{O}$ & $6.8860 \mathrm{E}-02$ & Мo-94 & $1.1470 \mathrm{E}-04$ \\
\hline $\mathrm{Al}_{2} \mathrm{O}_{3}$ Total & $1.0927 \mathrm{E}-01$ & Mo-95 & $1.9741 \mathrm{E}-04$ \\
\hline & $4.3706 \mathrm{E}-02$ & Мo-96 & $2.0683 \mathrm{E}-04$ \\
\hline $\mathrm{O}$ & $6.5560 \mathrm{E}-02$ & Mo-97 & $1.1842 \mathrm{E}-04$ \\
\hline $\mathrm{B}_{4} \mathrm{C}$ Total & $1.4415 \mathrm{E}-01$ & Mo-98 & $2.9921 \mathrm{E}-04$ \\
\hline B-10 & $1.0474 \mathrm{E}-01$ & Mo-100 & 1.1941E-04 \\
\hline B-11 & $1.0585 \mathrm{E}-02$ & Mn-55 & $1.7400 \mathrm{E}-03$ \\
\hline $\mathrm{C}$ & $2.8831 \mathrm{E}-02$ & $\mathrm{Si}-28$ & $1.5679 \mathrm{E}-03$ \\
\hline $\mathbf{N a}$ & $2.5423 \mathrm{E}-02$ & $\mathrm{Si}-29$ & 7.9614E-05 \\
\hline U10Zr Total & $4.0620 \mathrm{E}-02$ & $\mathrm{Si}-30$ & $5.2482 \mathrm{E}-05$ \\
\hline U-234 & 4.7949E-05 & & \\
\hline U-235 & $6.2863 \mathrm{E}-03$ & & \\
\hline $\mathrm{U}-238$ & $2.5173 \mathrm{E}-02$ & & \\
\hline Zr-90 & 4.6883E-03 & & \\
\hline Zr-91 & $1.0224 \mathrm{E}-03$ & & \\
\hline Zr-92 & $1.5628 \mathrm{E}-03$ & & \\
\hline $\mathrm{Zr}-94$ & $1.5837 \mathrm{E}-03$ & & \\
\hline Zr-96 & $2.5515 \mathrm{E}-04$ & & \\
\hline
\end{tabular}

From a geometric and material standpoint this core is very similar to the LANL design, but the lattice structure is quite different. Table 3 lists the main lattice/unit cell differences between these two cores. In the previous analysis of the LANL design, the core was found to be extremely sensitive to the web thickness, pitch, and clad thickness.

\section{Pitch and Clad Thickness}

To better understand the reactivity sensitivities of pitch and clad thickness (stainless steel mass) on the Design A and LANL cores, unit cell models were constructed with reflective boundary conditions to represent infinite lattice models which are a good approximation of the fuel and heat pipe arrays in the center of the Design A and LANL active cores. Figure 4 shows the unit cells for Design A and the LANL design. Figure 5 shows the three cases examined for Design A in which the outer SS clad thickness and the pitch (web thickness) were adjusted. Calculated k-infinity results are shown in Table 4 with Case 1 having the nominal Design A dimensions. Reducing the outer clad to $0.05 \mathrm{~cm}(0.5 \mathrm{~mm})$ in Case 2, but keeping the same pitch greatly increases the infinite multiplication factor. It is clear that reducing the SS 
outer clad will greatly reduce the amount of parasitic absorption by the steel cladding. In Case 3, the pitch is then reduced to eliminate the gap between unit cells and to simulate a comparable minimum web thickness with the LANL design. Again, the infinite multiplication factor increases by well over 1000 percent milli (pcm) and compares very similarly to the infinite LANL lattice. Decreasing the pitch boosts core reactivity through reduced axial leakage.

Table 3. Geometric differences between Design A unit cell and the LANL lattice structure.

\begin{tabular}{|l|c|c|}
\hline Lattice Dimensions (cm) & LANL & Design A \\
\hline Fuel-to-fuel web thickness & 0.175 & - \\
\hline Fuel-to-heat pipe web thickness & 0.100 & - \\
\hline Web thickness equivalent (SS only) & - & 0.2000 \\
\hline Web thickness equivalent (total) & - & 0.2128 \\
\hline Fuel-to-fuel pitch & 1.6 & - \\
\hline Fuel-to-heat pipe pitch & 1.6 & - \\
\hline Heat Pipe-to-heat pipe pitch & 2.7713 & 2.7860 \\
\hline
\end{tabular}
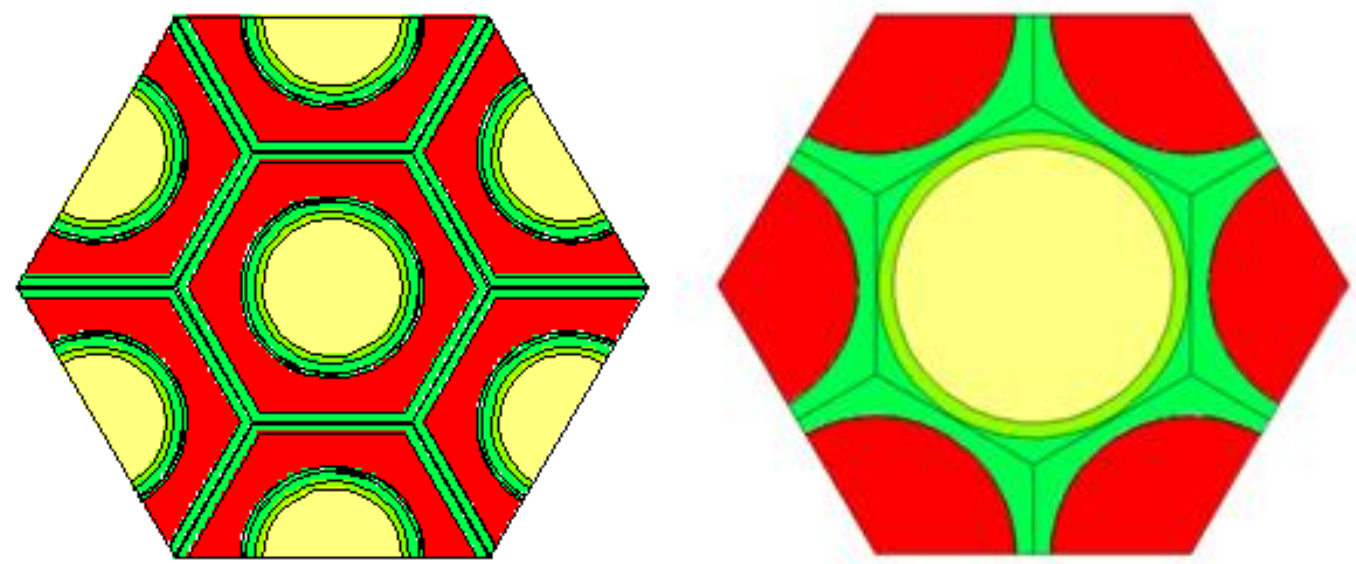

Figure 4. Design A (left) and LANL (right) unit cells with reflective boundary conditions for the infinite lattice models.
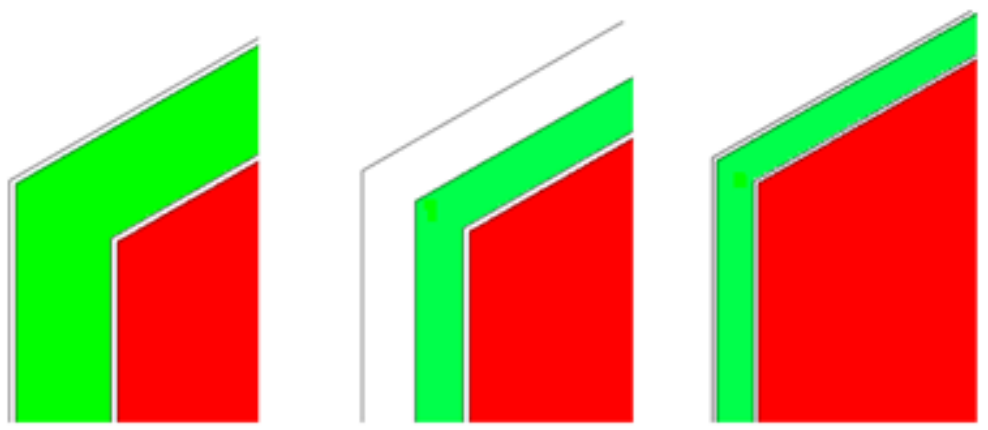

Figure 5. Three cases examined for Design A lattice.

Table 4. Calculated k-infinity results comparison.

\begin{tabular}{|c|l|l|l|l|l|c|}
\hline Case & $\begin{array}{l}\text { Outer SS } \\
\text { Clad }(\mathrm{cm})\end{array}$ & $\begin{array}{l}\text { Web thickness } \\
(\mathrm{cm})\end{array}$ & $\begin{array}{l}\text { Pitch } \\
(\mathrm{cm})\end{array}$ & $\begin{array}{l}\text { Fuel area per } \\
\text { unit cell }\left(\mathrm{cm}^{3}\right)\end{array}$ & $\begin{array}{l}\text { SS area per unit } \\
\text { cell }\left(\mathrm{cm}^{3}\right)\end{array}$ & $k_{\text {inf }}$ \\
\hline 1 & 0.10 & 0.2 & 2.786 & 2.900 & 1.6819 & 1.25953 \\
\hline 2 & 0.05 & 0.2 & 2.786 & 2.900 & 1.2103 & 1.27496 \\
\hline 3 & 0.05 & 0.1 & 2.686 & 2.900 & 1.2103 & 1.28830 \\
\hline LANL & - & 0.1 & 2.7713 & 3.132 & 1.5131 & 1.28501 \\
\hline
\end{tabular}




\section{Reactivity Control}

Twelve rotatable control drums are used for reactivity control during normal operations in both Design A and the LANL design. In both core designs, any 5 control drums will cause the core to go subcritical (any 4 nonadjacent drums can also achieve this). Additionally, there is both an annular and a solid $\mathrm{B}_{4} \mathrm{C}$ emergency shutdown rod (again 90\% enriched B-10) that can be inserted through the central channel of the core. These rods lie beneath the core and provide independent mechanisms to shut the reactor down in an emergency situation. The terminology 'all poisons in' and 'all poisons out' refers to all 12 control drums and both emergency shutdown rods inserted into the core. Table 5 gives the calculated core keffectives for the different reactivity control conditions. Each of the three reactivity control mechanisms can independently bring the core to a sufficiently subcritical state with a shutdown margin of around 5\% $\left(k_{\text {eff }} \sim 0.95\right)$ with just the 12 control drums.

Table 5. Reactivity control

\begin{tabular}{|l|l|l|}
\hline \multirow{2}{*}{ Control Condition/Parameter } & \multicolumn{2}{|c|}{$k_{\text {eff }} \pm 0.00002$} \\
\cline { 2 - 3 } & Design A & LANL $^{2}$ \\
\hline All Poisons Out & 1.02825 & 1.02153 \\
\hline All Poisons In & 0.84594 & 0.82500 \\
\hline Control Drums In & 0.95042 & 0.92602 \\
\hline Annular Shutdown Rod In & 0.94555 & 0.94211 \\
\hline Solid Shutdown Rod In & 0.95933 & 0.95601 \\
\hline & \multicolumn{2}{|c|}{$\beta=0.007$} \\
\hline BOL Excess Reactivity (\$) & 3.92 & 2.88 \\
\hline Total Drum Worth $(\$)$ & 11.38 & 14.42 \\
\hline Individual Drum Worth (\$) & 0.97 & 1.21 \\
\hline Critical Control Drum Rotation $\left({ }^{\circ}\right)$ & 65 & 48 \\
\hline Annular Shutdown Rod Worth $(\$)$ & 12.15 & 11.79 \\
\hline Solid Shutdown Rod Worth $(\$)$ & 9.98 & 958 \\
\hline
\end{tabular}

\section{Reactivity Feedback}

There are multiple negative reactivity feedback effects in both Design A and the LANL design. The primary effect results from the Doppler broadening of the low-enriched $\mathrm{UO}_{2}$ fuel. As the fuel temperature increases, the neutron resonances will broaden increasing the effective neutron absorption in the core. Figure 6 shows how the calculated core k-effective decreases as the fuel temperature increases. Three other negative reactivity feedback effects contribute to the overall total temperature coefficient of reactivity. These additional effects include (1) thermal expansion of the fuel, (2) alumina reflector radial thermal expansion, and (3) outer SS fuel clad thermal expansion. The fuel thermal expansion was examined in the axial direction at $1 \mathrm{~mm}$ intervals for a total elongation of $1.5 \%$ of the cold length. Figure 7 shows how the core k-effective decreases when the fuel elongates. As the fuel lengthens, the volume increases which ultimately reduces the $\mathrm{UO}_{2}$ number density. A reduction in material number density is also seen in the alumina reflector and SS cladding due to thermal expansion. This results in an increase in the leakage and an increase in the parasitic absorption, respectively. Table 6 lists the worth per degree centigrade of temperature increase for each feedback mechanism, each of which was calculated independently. The total negative feedback is very comparable to the LANL reference case shown in Table 6. The latter is slightly more negative due to the swelling of the SS monolith structure compared to that of the outer SS clad in Design A.

\footnotetext{
${ }^{2}$ As calculated by INL.
} 


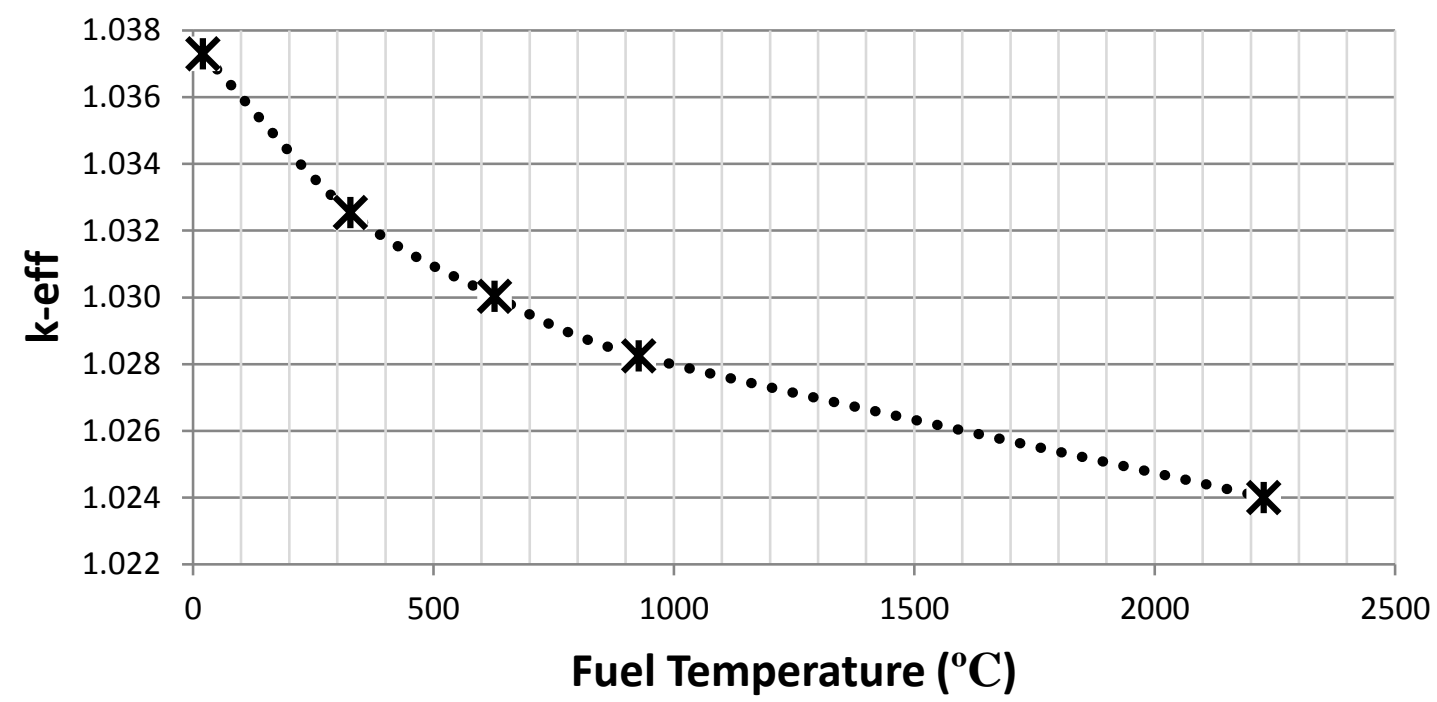

Figure 6. Negative reactivity effect due to U-238 Doppler-broadening in the $\mathrm{UO}_{2}$ fuel.

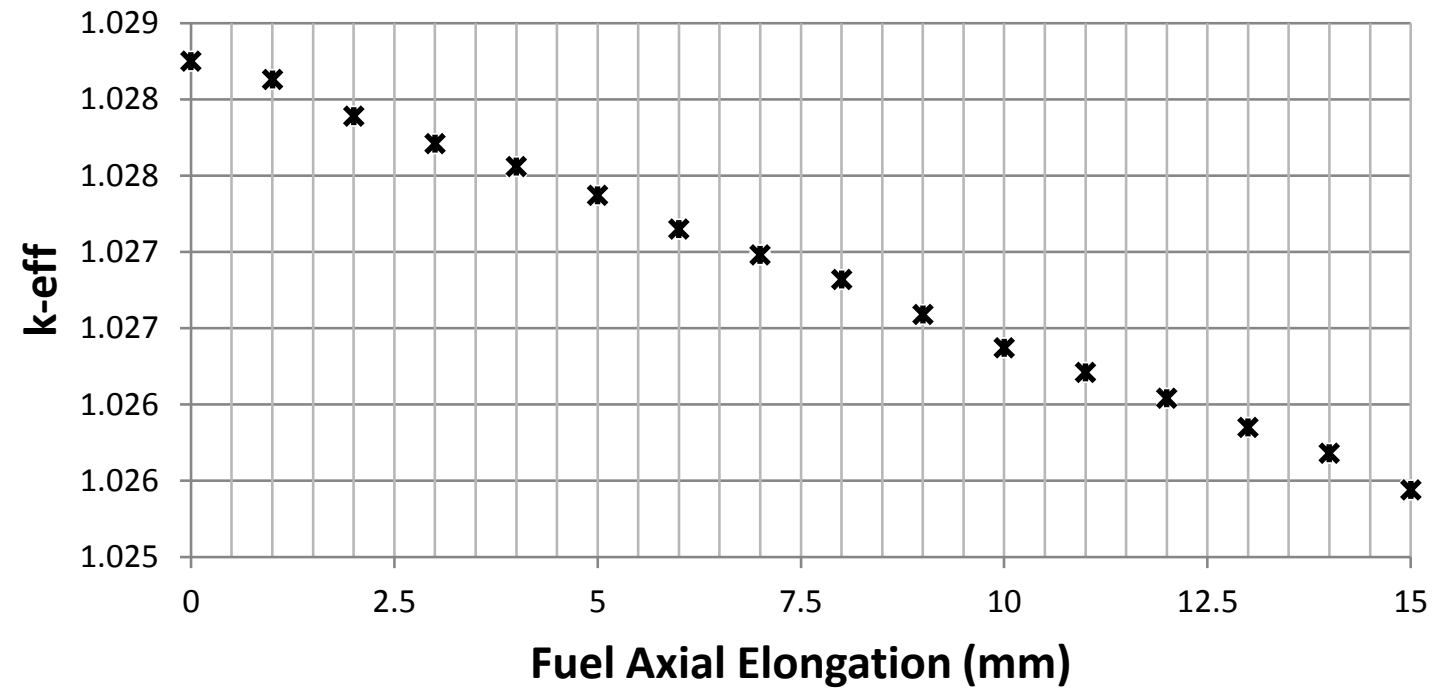

Figure 7. Negative reactivity feedback due to axial elongation of the $\mathrm{UO}_{2}$ fuel.

Table 6. Magnitude of the temperature coefficients of reactivity feedback effects.

\begin{tabular}{|l|l|l|}
\hline Feedback Effect $\left(\mathrm{cents}^{\circ}{ }^{\circ} \mathrm{C}\right)$ & Design A & LANL \\
\hline Doppler & -0.1074 & -0.1011 \\
\hline $\mathrm{UO}_{2}$ Fuel Axial Elongation & -0.0422 & -0.0408 \\
\hline Alumina Reflector Radial Thermal Expansion & -0.0225 & $-0.0225^{3}$ \\
\hline Outer SS Fuel Clad Thermal Expansion & -0.0323 & - \\
\hline SS Monolith Thermal Expansion & - & -0.06034 \\
\hline
\end{tabular}

\footnotetext{
${ }^{3}$ This parameter was not directly calculated for the LANL design. However, the alumina reflector has the exact same geometry and material properties in both designs and is thus assumed to have a very comparable effect.
} 


\section{Total}

$-0.2044$

$-0.2247$

\section{Water Ingress (Flooding)}

Although there is very little chance that a reactor of this design could flood with water, this situation becomes a possibility during the transportation of such a reactor. Since the bulk of the reactor is expected to be assembled before shipment, several scenarios were examined to better understand the core behavior should this occur. The water ingress analysis was conducted for the Design A core and is compared to similar calculations for the LANL design. Table 7 shows the calculated core k-effective for different flooding situations.

If the core were to simply submerge in water (become fully surrounded) there is very little change in the reactivity. This is because the $\mathrm{B}_{4} \mathrm{C}$ shield will absorb most all neutrons that radially leak out of the core, and any neutron that happens to escape and reflect back towards the core is extremely likely to still be absorbed in the outer $\mathrm{B}_{4} \mathrm{C}$ neutron shield or the $\mathrm{B}_{4} \mathrm{C}$ arcs in the control drums rather than re-enter the active core. The $\mathrm{BeO}$ axial reflectors already provide very efficient axial reflection, thus having water above or below the core has a very minimal impact.

The second flooding scenario involves all of the gaps and voids filling with water. This greatly increases the core reactivity due to the vast thermalization of neutrons that takes effect. However, for this to occur the outer SS core barrel or one of the upper or lower SS grid plates would have to be penetrated, making this an unlikely situation.

The third flooding scenario, although again unlikely, examines the effect when the central void channel (emergency shutdown rods out of core) fills with water. A very interesting and unexpected large decrease in the core reactivity takes place. As seen in Figure 8, the center of the central channel (center of the core) of both designs exhibits a very hard neutron spectrum. The neutron energy cutoff occurs around $1 \mathrm{keV}$, which is to be expected in a fast reactor. However, when the central channel fills with water the neutron spectrum undergoes a substantial shift to thermal energies, peaking approximately 6 orders of magnitude less in energy. Figure 8 shows the flux at the core midplane in the central void for the emergency shutdown rods when the area is both flooded and voided. To help verify this phenomenon, Design B was also examined and similar results are shown in the figure. Thus, under normal operation (central channel voided) the vast majority of neutrons that enter the central channel will simply stream through unimpeded and enter fuel on the opposite side of the core (some will leak out axially or become absorbed in the SS structure). However, if this channel fills with water then a large percentage of the neutrons that would normally stream through to the other side to produce additional fissions will instead be thermalized and parasitically absorbed. The channel hexagon flat-to-flat measures roughly $19 \mathrm{~cm}$, and the mean free path of a thermal neutron in water is approximately $0.3 \mathrm{~cm}$ [6]. As seen in Table 7, this effect is actually enhanced when in combination with the other two rather than competing. Since both the second and third flooding scenarios would have to be initiated due to a puncture to the outer core, it appears that the reactor core would remain subcritical following a full core ingress of water.

The fourth and final flooding scenario analyzed was the core behavior if the heat pipes also became flooded. Assuming a heat pipe flooded length in the active core of $150 \mathrm{~cm}$, this would amount to approximately $292 \mathrm{~cm}^{3}$ of water per heat pipe. Although the location of the heat pipe will affect the magnitude of this reactivity insertion, Table 7 clearly shows the flooding of heat pipes will be a positive reactivity insertion. If the core submerges under water and all gaps, voids, and the central channel flood, then the core can go critical if 36 or more heat pipes flood. This is with all control poisons removed from the core, and a similar flooding scenario with the LANL design requires 27 flooded heat pipes to go critical. If all poisons were inserted in the core, then Design A would go critical if 77 heat pipes flooded. 
Table 7. Design A Core response due to water ingress.

\begin{tabular}{|l|l|l|l|c|}
\hline \multirow{2}{*}{ Flooding Scenario } & \multicolumn{2}{c|}{ Design A } & \multicolumn{2}{c|}{ LANL } \\
\cline { 2 - 5 } & $k_{\text {eff }} \pm 0.00002$ & $\Delta k_{\text {eff }}(\mathrm{pcm})$ & $k_{\text {eff }} \pm 0.00002$ & $\Delta k_{\text {eff }}(\mathrm{pcm})$ \\
\hline Core (no water) & 1.02825 & - & 1.02153 & - \\
\hline (1) Core submerges in water & 1.02845 & +20 & & \\
\hline (2) Gaps and voids flood with water & 1.04139 & +1314 & 1.04496 & +2343 \\
\hline$(1)+(2)$ & 1.04150 & +1324 & 1.04526 & +2373 \\
\hline$(3)$ Central channel floods & 0.96666 & -6159 & 0.96563 & -5590 \\
\hline$(1)+(2)+(3)$ & 0.96448 & -6377 & & \\
\hline$(1)+(2)+(3)+1$ heat pipe & 0.96514 & -6311 & & \\
\hline$(1)+(2)+(3)+2$ heat pipes & 0.96584 & -6241 & & \\
\hline$(1)+(2)+(3)+3$ heat pipes & 0.96660 & -6165 & & \\
\hline$(1)+(2)+(3)+5$ heat pipes & 0.96918 & -5907 & & \\
\hline$(1)+(2)+(3)+10$ heat pipes & 0.97252 & -5573 & & -2153 \\
\hline$(1)+(2)+(3)+20$ heat pipes & 0.98256 & -4569 & & \\
\hline$(1)+(2)+(3)+27$ heat pipes & - & - & & \\
\hline$(1)+(2)+(3)+30$ heat pipes & 0.99445 & -3380 & & \\
\hline$(1)+(2)+(3)+36$ heat pipes & \multicolumn{1}{|c|}{ critical } & -2825 & & \\
\hline
\end{tabular}

The Design A core has only $\$ 3.92$ of excess reactivity and multiple negative feedback mechanisms to ensure safe operation. If additional reactivity were needed for any reason, the core layout permits the addition of 1 entire ring of fuel/heat pipe elements (72) without altering the alumina reflector or control drums. Figure 9 shows that with an additional 72 elements added to the periphery of the core, the excess reactivity is boosted to nearly $\$ 5.83$. Other ways to increase the core reactivity include decreasing the B10 enrichment in the control drum arcs or increasing the alumina reflector outer radius (while at the same time pushing the control drums further out radially). Although these two parametric studies were not specifically analyzed for Design A, the analysis was performed for the LANL design and the results should be very similar [1]; amounting to about a $500 \mathrm{pcm}$ boost for a decrease in B-10 enrichment from $90 \%$ to $30 \%$, and about a $400 \mathrm{pcm}$ increase for increasing the radial dimension of the alumina side reflector by $2.5 \mathrm{~cm}$.

If liquid metal sodium or potassium were to be used to thermal bond the fuel elements in Design A, filling the gap between fuel elements, the core excess reactivity would change by less than $\$ 0.06$. This is a negligible core reactivity change. Thermal bonding with liquid metal would not affect the core reactivity. 


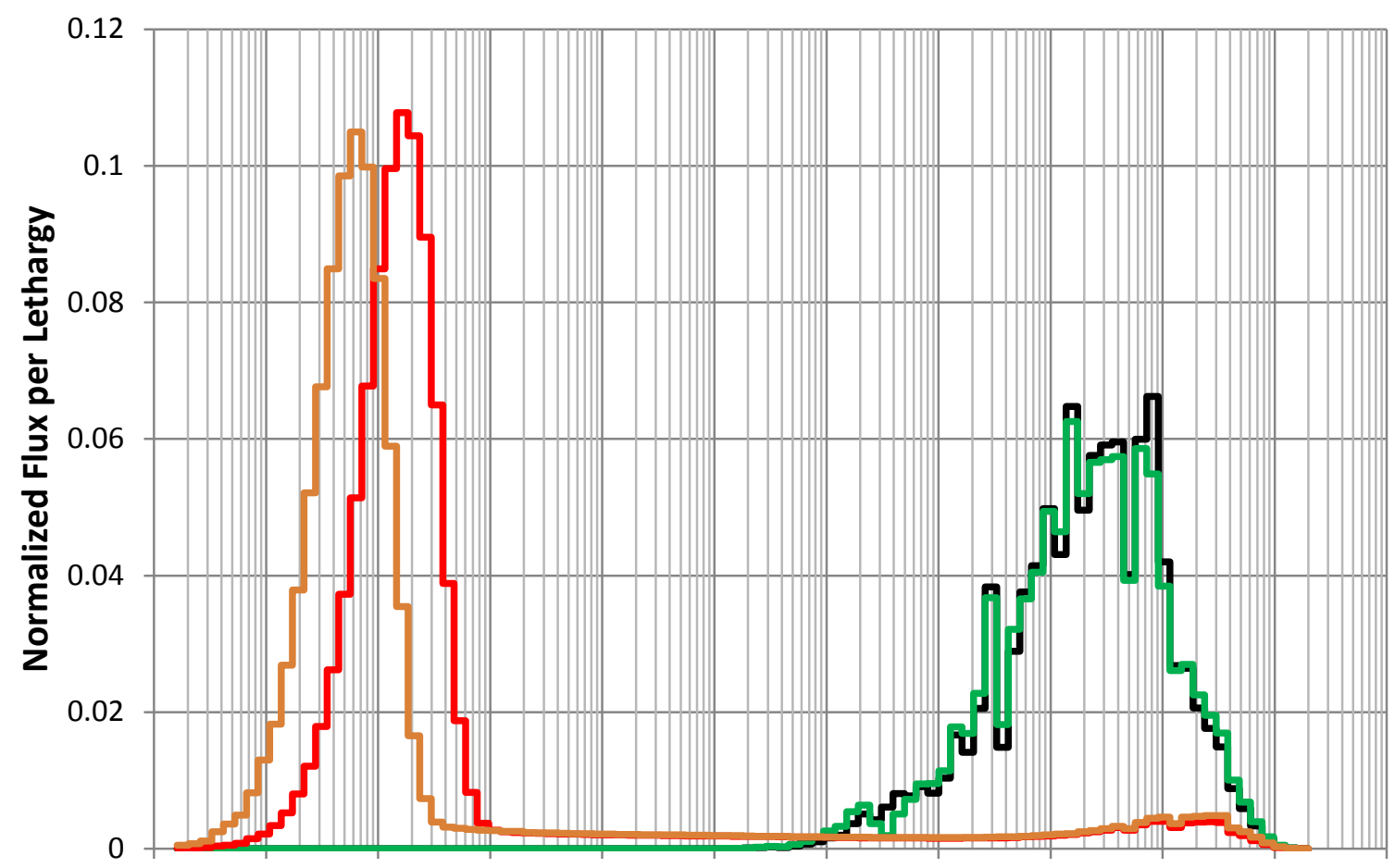

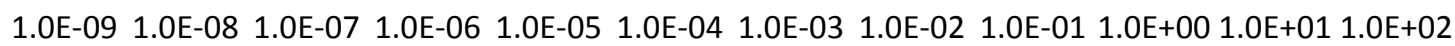
Energy (MeV)

$\longrightarrow$ Design A (void) —Design B (void) —Design A (water) —Design B (water)

Figure 8. Normalized flux spectrum in the center of the central channel for Designs A and B.

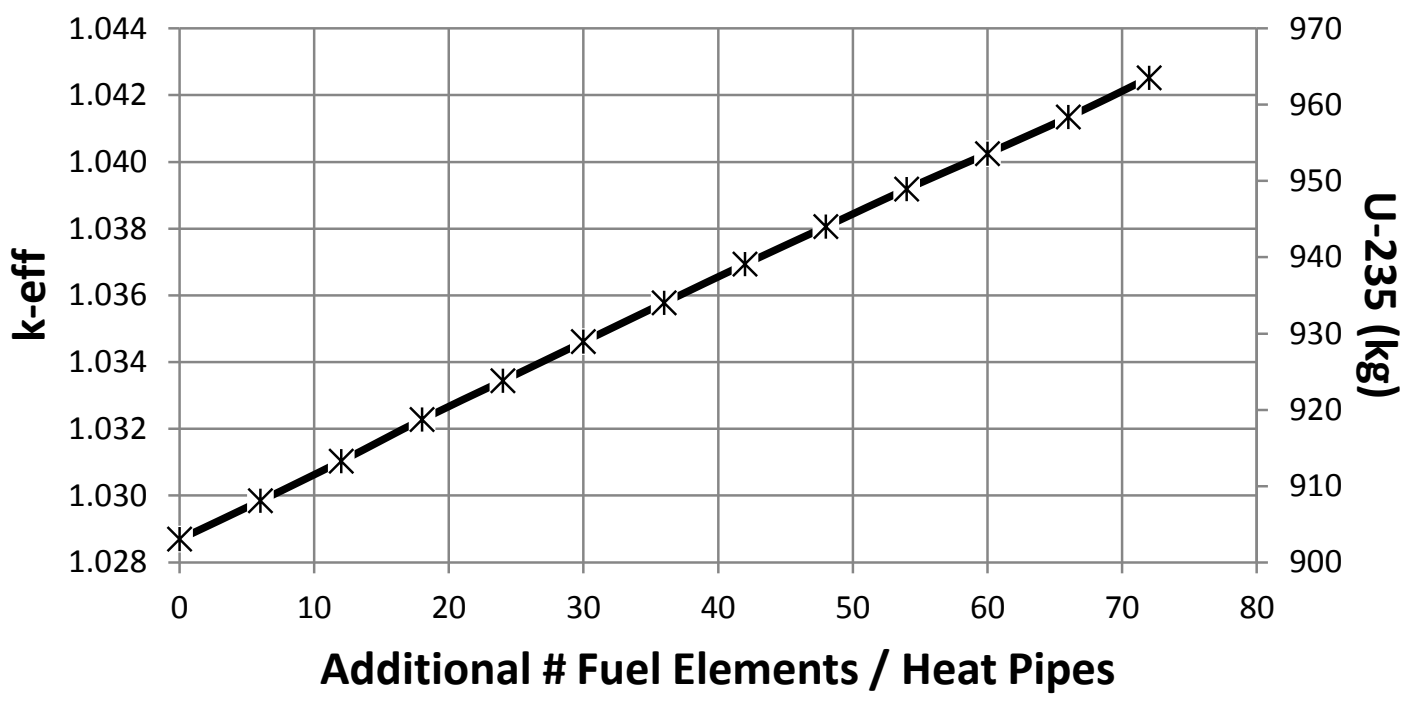

Figure 9. Calculated core k-effective and U-235 mass loading as a function of additional fuel elements on the outer core periphery. 


\section{Power and Burnup Estimates}

The F7 tally (energy deposition tally) in MCNP was used to calculate the heat generation rates and pin powers. Since there are 978 fewer fuel elements in the Design A core, the pin powers are higher than those in the LANL design. However, the peak-to-average pin power is less in Design A (Figure 10). The pin power peaking factor is estimated to be 1.23 for the hottest pin. The increase in power at the bottom of the pin is due to the solid $\mathrm{BeO}$ lower axial reflector (there is much less neutron reflection at the top of the core because the upper $\mathrm{BeO}$ reflector is punctuated by the heat pipes).

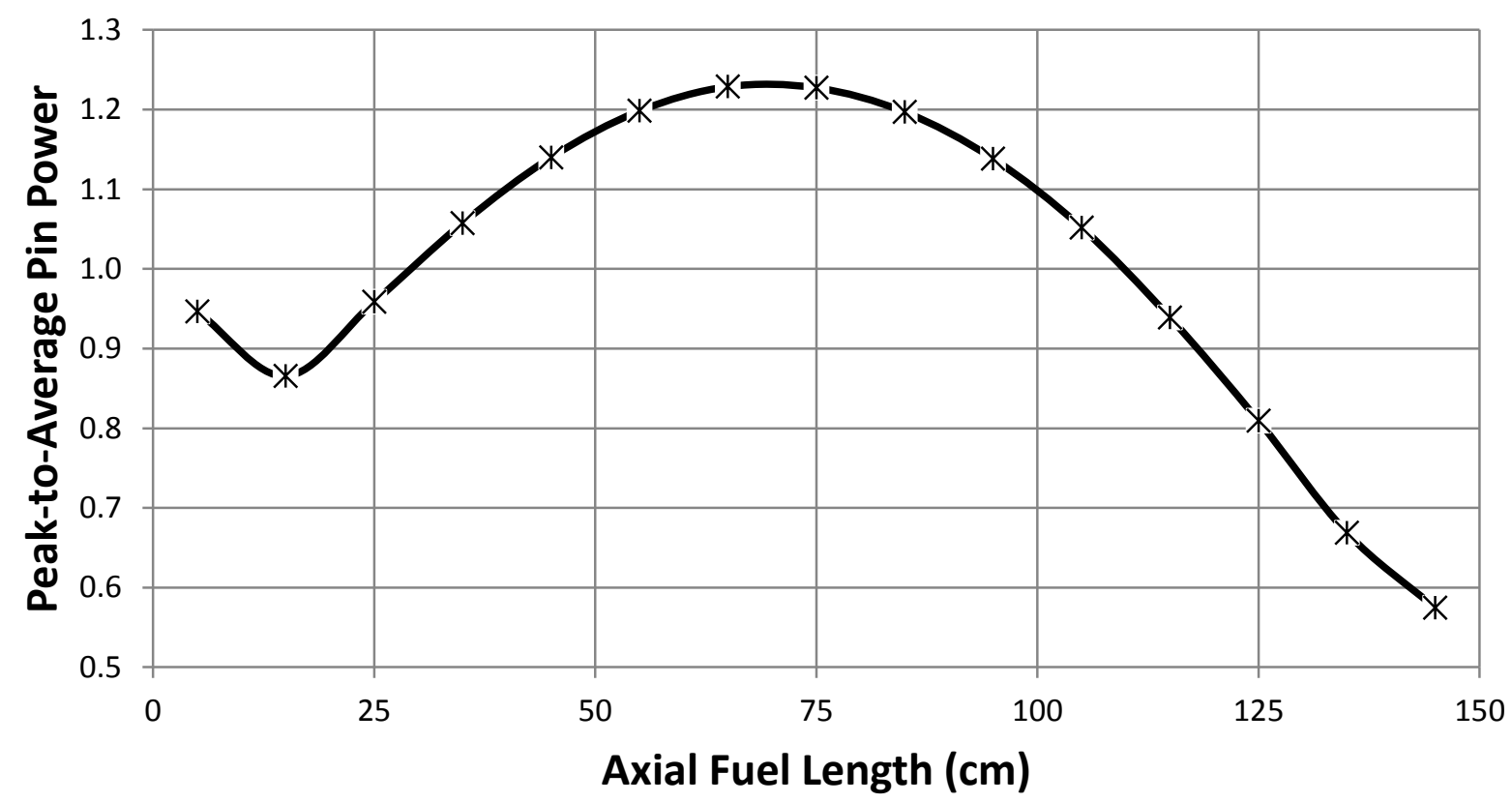

Figure 10. Axial peak-to-average power profile in the hottest pin (Design A).

Table 8 gives the calculated fuel element power performance parameters and the core burnup characteristics. The results are very similar to the LANL design. Both cores will experience very small burnups over a 5 year power cycle and a corresponding small reactivity swing. In order to calculate the $\%$ U-235 depletion, fission density, and fissions per initial heavy metal atom (FIMA), a single fuel pin in an infinite lattice model was depleted using the SCALE6.2 package [7]. A single pin model (as seen in Figure 1) was deemed appropriate due to the very low burnup and minimal reactivity swing as seen in Figure 11. SCALE consists of different modules that are coupled together through the control module TRITON to efficiently and easily perform a burnup calculation. The KENO6 Monte Carlo transport solver was used to perform the core eigenvalue calculation, and this data is then passed to the ORIGEN module which solves the Bateman equations to obtain burnup dependent isotopics. These isotopics are then used at the next time step to continue the process until the desired burnup is reached. Equations (1) (3) were then used to calculate the \%U-235 depletion, fission density, and FIMA with $N$ being the total atom density at time $t_{0}=$ initial and $t_{f}=$ final, and $H M=$ heavy metal. All initial heavy metal is ${ }^{234} \mathrm{U},{ }^{235} \mathrm{U}$, and ${ }^{238} \mathrm{U}$, and $N_{H M}^{\text {final }}$ includes all heavy metal isotopes larger than ${ }^{228} \mathrm{Th}$. 
Table 8. Design A and LANL thermal and core burnup parameters.

\begin{tabular}{|l|l|l|}
\hline & Design A & \multicolumn{1}{|c|}{ LANL $^{4}$} \\
\hline Number of fuel pins & \multicolumn{1}{|c|}{1134} & \multicolumn{1}{|c|}{2112} \\
\hline Average pin power $(\mathrm{kW})$ & 4.41 & 2.37 \\
\hline Max pin power $(\mathrm{kW})$ & 5.51 & 3.55 \\
\hline Peak-to-average & 1.25 & 1.50 \\
\hline Pin peaking factor $($ axial) & 1.23 & 1.29 \\
\hline Peak linear heat rate $(\mathrm{kW} / \mathrm{m})$ & 3.67 & 2.37 \\
\hline Average power density $\left(\mathrm{W} / \mathrm{cm}^{3}\right)$ & 10.14 & 9.90 \\
\hline Peak power density $\left(\mathrm{W} / \mathrm{cm}^{3}\right)$ & 12.66 & 14.9 \\
\hline $\mathrm{U}$ mass $(\mathrm{kg})$ & 4573 & 4600 \\
\hline U-235 mass $(\mathrm{kg})$ & 903 & 908 \\
\hline Specific power $(\mathrm{MW} / \mathrm{tHM})$ & 1.093 & 1.087 \\
\hline Average Burnup $(\mathrm{GWd} / \mathrm{t})$ & 2.0 & 2.0 \\
\hline$\%$ U-235 depletion & 1.26 & 1.0 \\
\hline FIMA $(\%)$ & 0.22 & 0.33 \\
\hline Peak fission density $\left(\right.$ fissions $\left./ \mathrm{cm}^{3}\right)$ & $5.14 \mathrm{E}+19$ & $7.80 \mathrm{E}+19$ \\
\hline
\end{tabular}

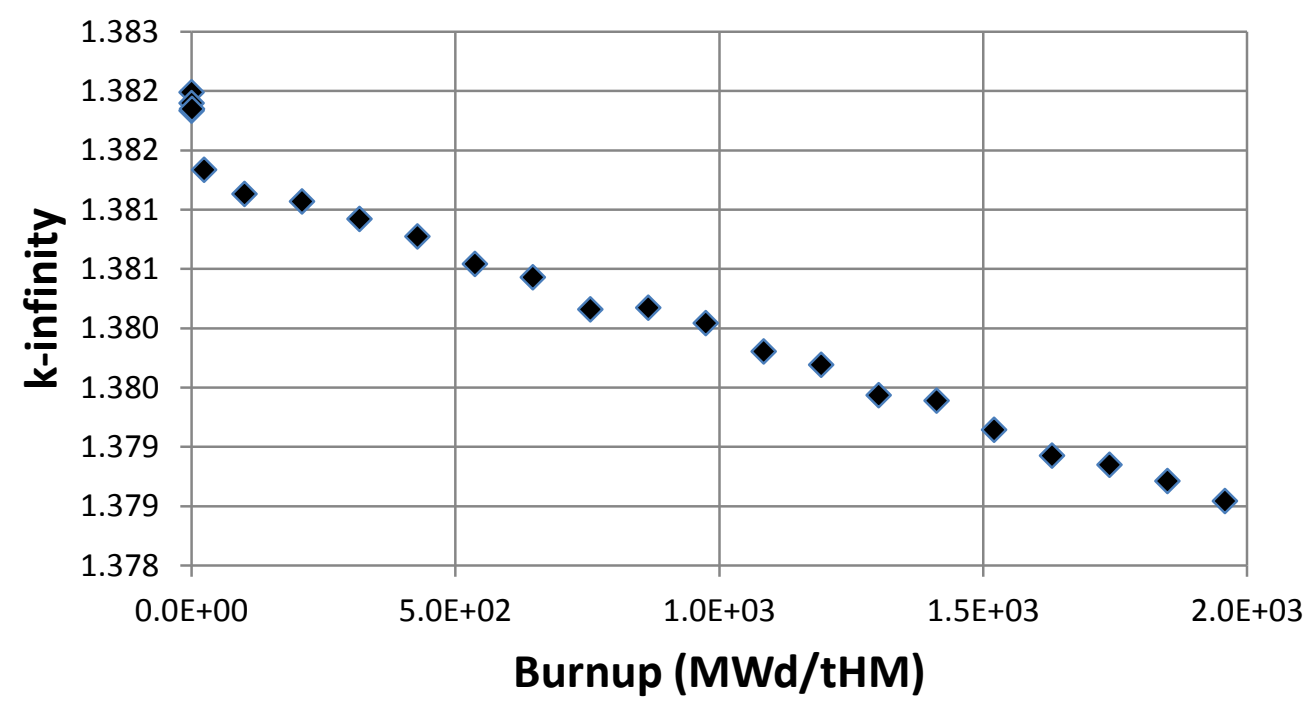

Figure 11. Single fuel element depletion over 5 years (infinite lattice).

$$
\begin{gathered}
\%{ }^{235} U_{\text {depletion }}=100 * \frac{N_{U 235}^{\text {initial }}-N_{U 235}^{\text {final }}}{N_{U 235}^{\text {initial }}} \\
F I M A=100 * \frac{N_{H M}^{\text {initial }}-N_{H M}^{\text {final }}}{N_{H M}^{\text {initial }}} \\
F D=1 * 10^{24} *\left(N_{H M}^{\text {initial }}-N_{H M}^{\text {final }}\right)
\end{gathered}
$$

\footnotetext{
${ }^{4}$ These parameters were calculated according to the INL model of the LANL design and may differ slightly from those reported by LANL.
} 


\section{Decay Heat}

After each ORIGEN depletion calculation SCALE stores the cross-section libraries (per user specified isotopes) at the given burnup state point. Once the calculation is completed, these state points are combined onto a single (.f33) file. These libraries can then be used with the similarly generated (.f71) isotopic file to generate decay heat curves ${ }^{5}$. These curves are shown in Figure 12 over different time scales. As is typical for nuclear reactors, the initial power drops to around $6.7 \%$ of the operating level, amounting to roughly $73 \mathrm{~kW}$. After 1 day of cooling the decay heat generates about $5 \mathrm{~kW}$, and after 1 year the power drops to less than $0.4 \mathrm{~kW}$. Figure 13 shows the fractional contribution of the decay heat attributed to minor actinides (MAs) and fission products (FPs). The FPs clearly dominate the source of decay heat and are not surpassed until after 140 years, at which point in time the decay heat is negligible. Table 9 lists the largest isotopic contributor for both the MAs and FPs.

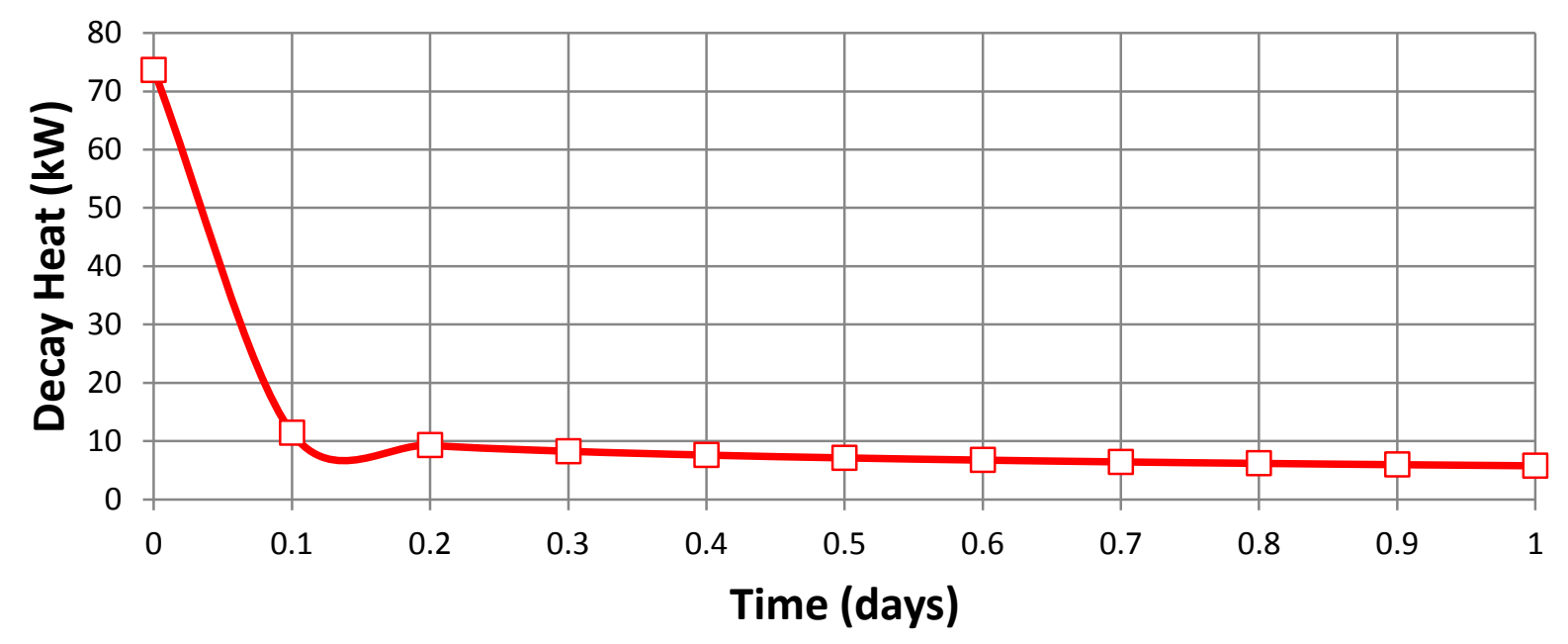

Figure 11. Design A total core decay heat following shutdown.

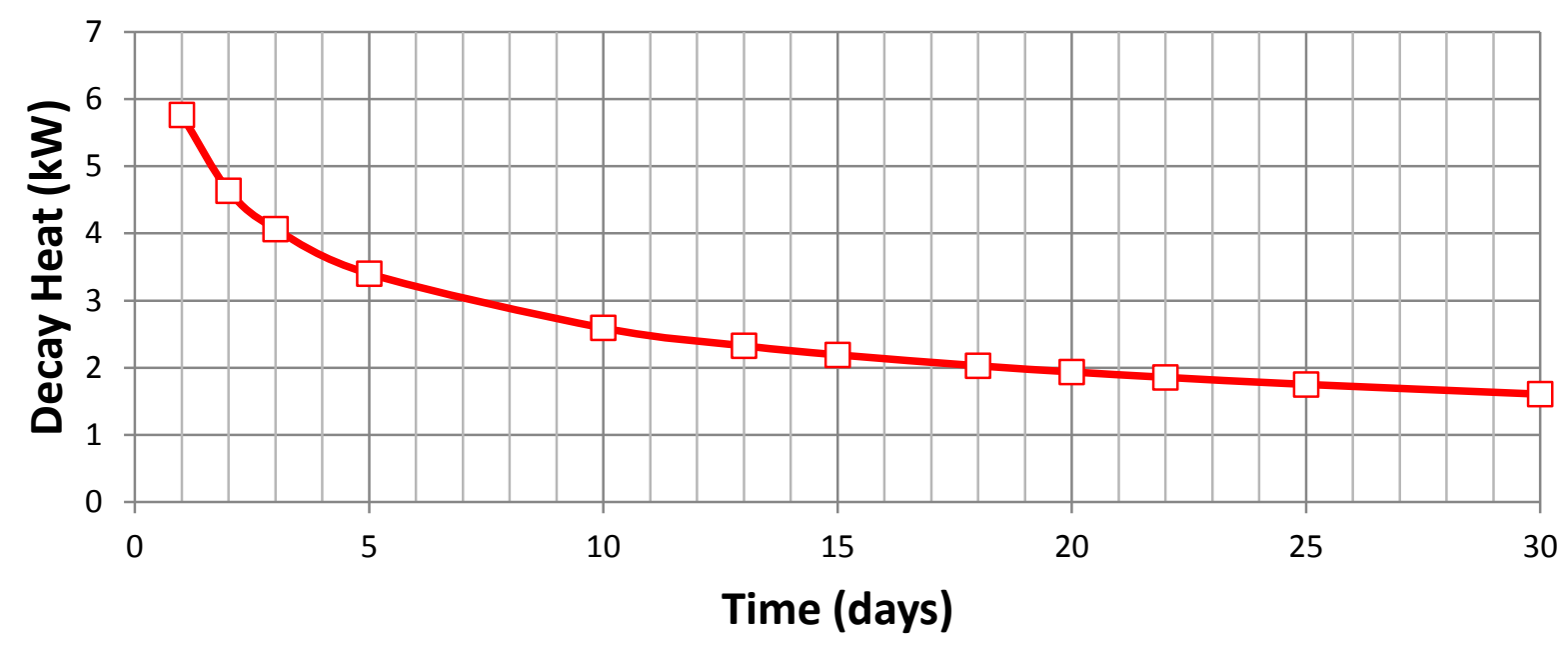

${ }^{5}$ A very similar approach (i.e. infinite single pin depletion for decay heat analysis) has been done at JAEA [8]. 

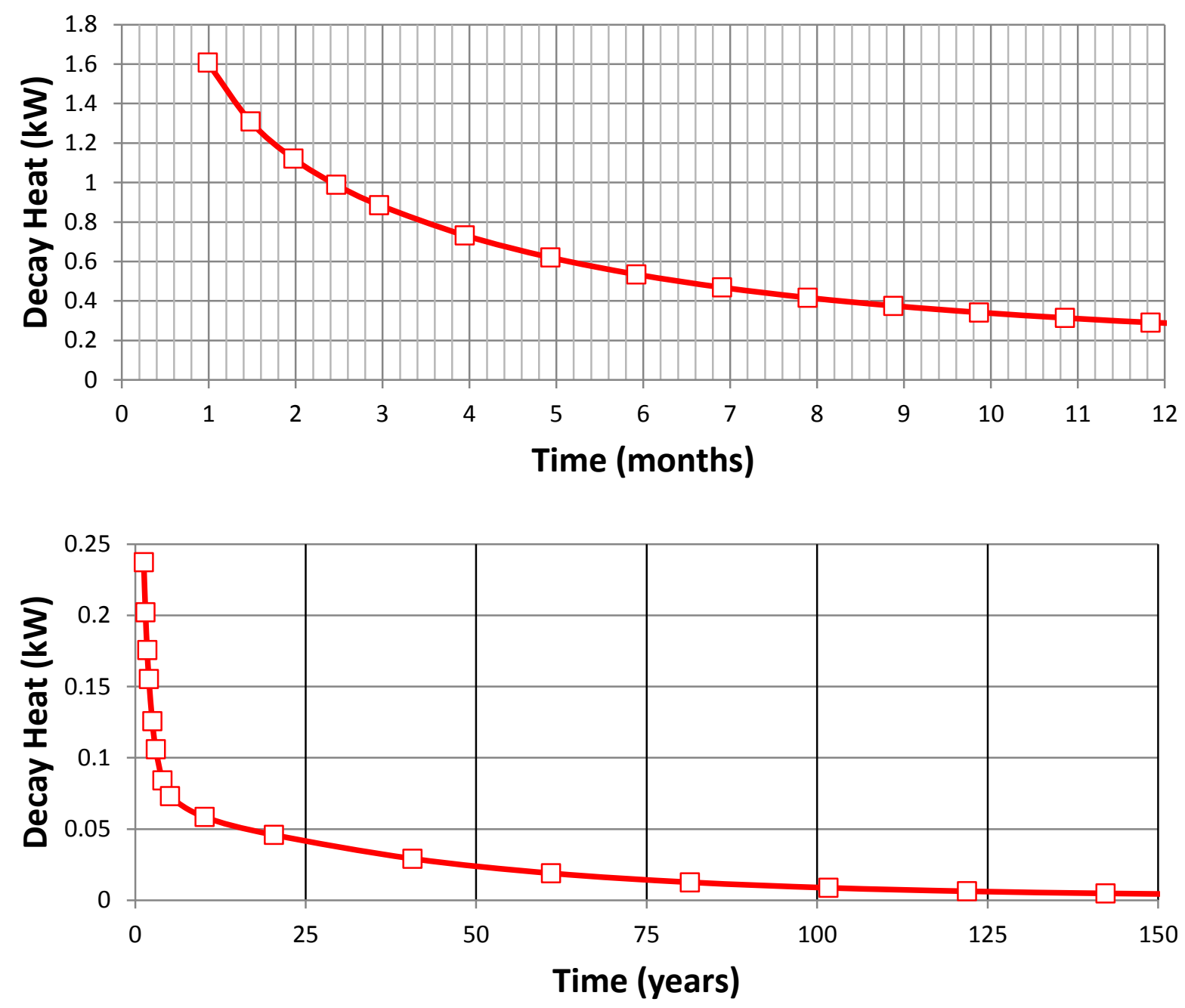

Figure 12. Design A total core decay heat following shutdown.

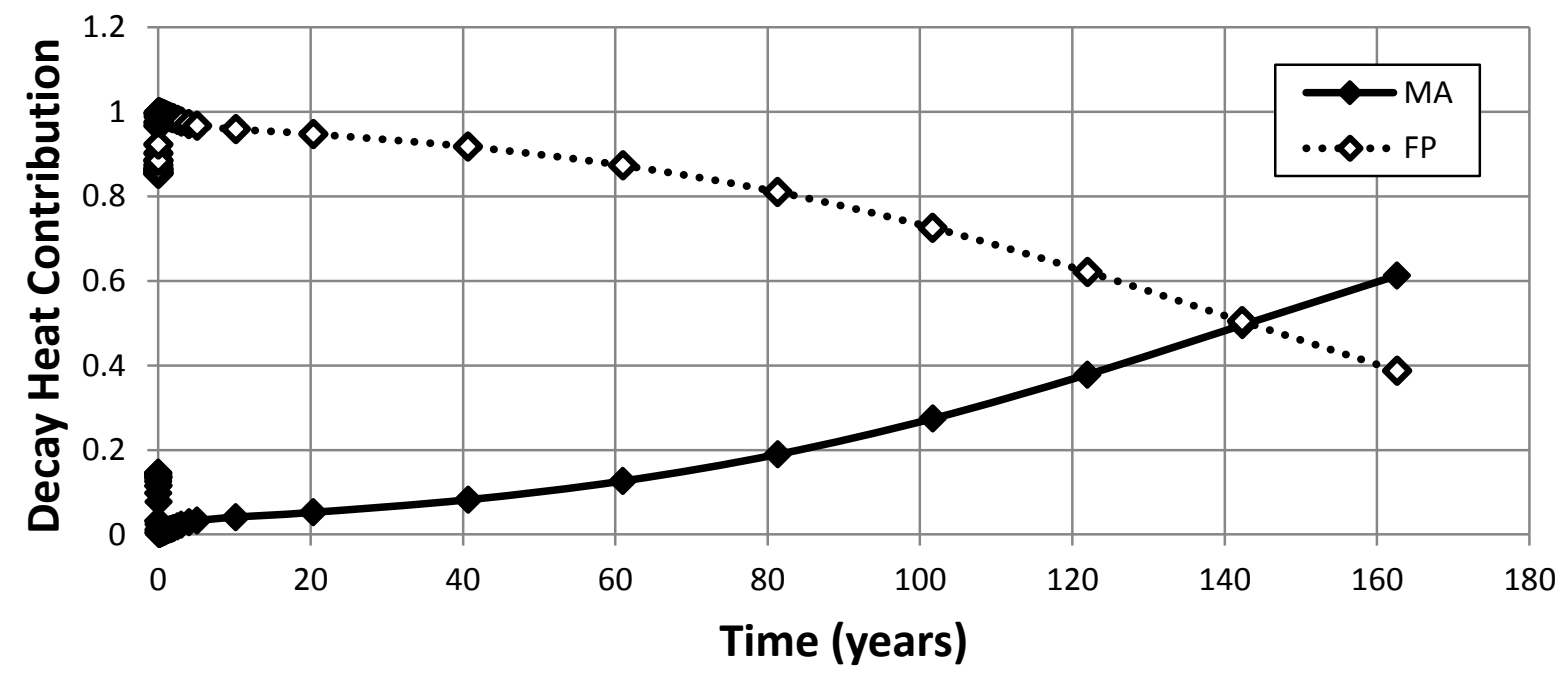

Figure 13. Fractional decay heat contribution from minor actinides (MAs) and fission products (FPs). 
Table 9. Largest isotopic contributor to the decay heat generation (Design A).

\begin{tabular}{|l|c|c|c|c|}
\hline & \multicolumn{2}{|c|}{ Fission Product } & \multicolumn{2}{c|}{ Minor Actinide } \\
\hline Decay Time & Isotope & $\%$ & Isotope & $\%$ \\
\hline 0 & $\mathrm{I}-134$ & 1.8 & $\mathrm{U}-239$ & 1.7 \\
\hline 1 day & $\mathrm{La}-140$ & 16.5 & $\mathrm{~Np}-239$ & 14.5 \\
\hline 1 month & $\mathrm{Pr}-144$ & 20.9 & $\mathrm{Pu}-239$ & 0.13 \\
\hline 1 year & $\mathrm{Pr}-144$ & 51.6 & $\mathrm{Pu}-239$ & 0.72 \\
\hline 10 years & $\mathrm{Y}-90$ & 43.6 & $\mathrm{Pu}-239$ & 3.6 \\
\hline 100 years & $\mathrm{Y}-90$ & 32.3 & $\mathrm{Pu}-239$ & 23.9 \\
\hline 160 years & $\mathrm{Y}-90$ & 16.7 & $\mathrm{Pu}-239$ & 53.5 \\
\hline
\end{tabular}

\section{Dose Rates}

Finally, the neutron and photon dose rates were calculated at various places outside the core. These were originally done with the MCNP F5 point detector tally due to the ease of use. However, these ended up requiring vast amounts of additional computation time. Thus the F4 tally was used instead, since the two yielded very similar results. In conjunction with each F4 tally, both the Dose Energy (DE) and Dose Function (DF) MCNP cards were used. This allows for a flux-to-dose conversion factor to modify the F4 tally, and the NCRP-38, ANSI/ANS-6.1.1-1977 neutron and photon flux-to-dose conversion factors were used as listed in the MCNP6.1 User Manual [9]. Figure 14 represents the dose rate outside of the outer radial $15.24 \mathrm{~cm}$ thick $\mathrm{B}_{4} \mathrm{C}$ shield at the mid-plane of the reactor core. Tallies were taken in incremental distances for both neutrons and photons. The neutron dose rate is approximately 1 order of magnitude greater than the photon dose rate. The total dose rate drops less than an order of magnitude 1.0 meter outside the reactor shield. This could be reduced by increasing the $\mathrm{B}_{4} \mathrm{C}$ shield thickness or providing a thick concrete biological shield to protect personnel and prevent neutron activation of the surrounding soil and structures. The U.S. NRC has set the occupational total effective dose equivalent limit for adults at 5 rem/year [10]. The calculated dose 1 meter outside of the reactor shield is 4 orders of magnitude larger than this. Therefore, the core almost certainly would have to be encased in a biological shield. 


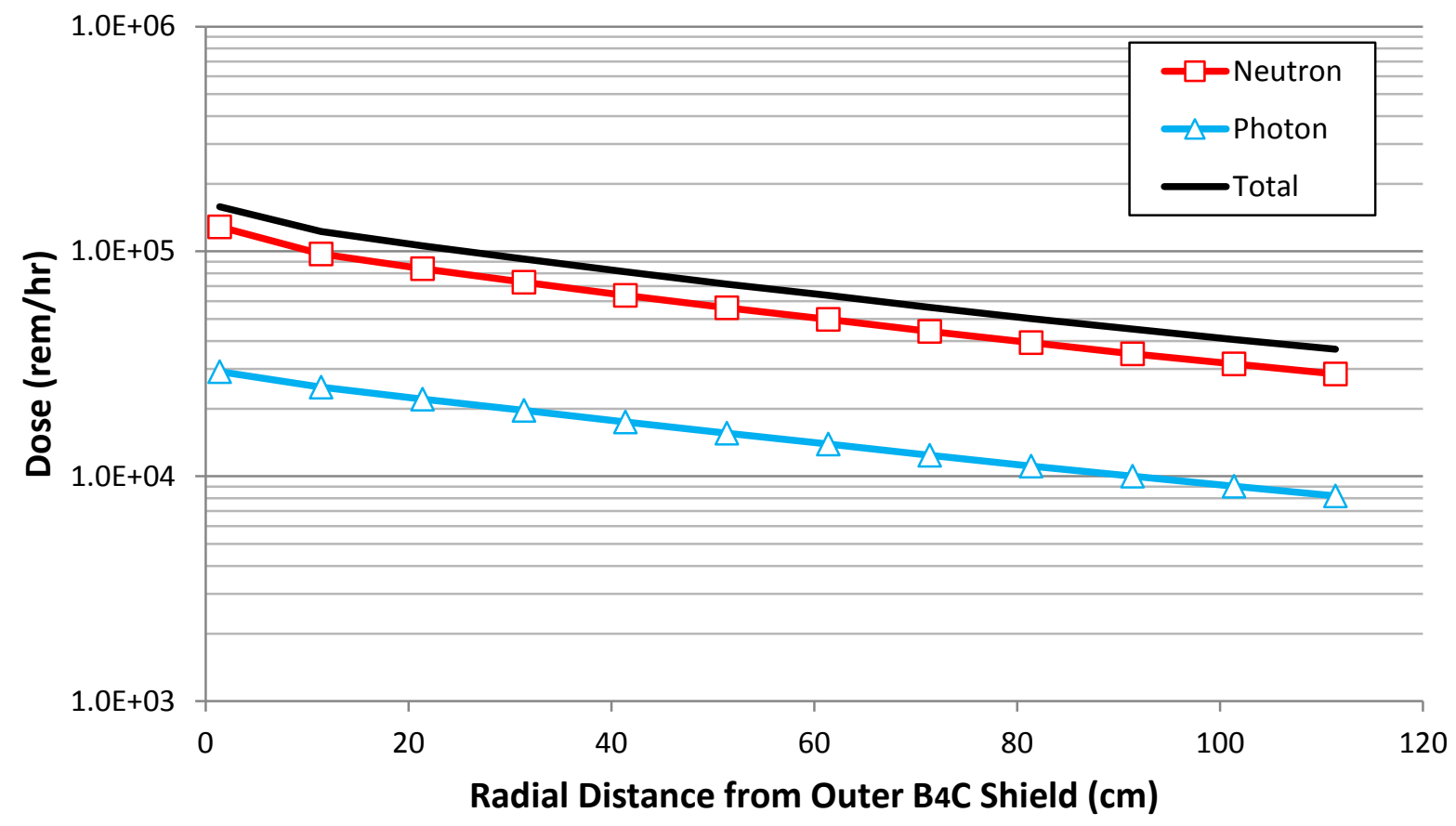

Figure 14. Neutron and photon dose rates at the core mid-plane outside of the reactor shield.

The dose rate was also examined above the core. Neutrons and photons can readily stream from the core up-through the heat pipes and activate structures above the core (e.g. decay heat exchanger, primary heat exchanger, heat pipe condenser). The dose rate is approximately 4 orders of magnitude greater above the core than radially outside $\mathrm{B}_{4} \mathrm{C}$ side shields. The neutron dose rate alone is about 2 orders of magnitude greater than the photon dose rate.

Two different shields were examined to lower the dose. As seen in Figure 15, if $9 \mathrm{~cm}$ of $5 \mathrm{wt} \%$ borated polyethylene and $1 \mathrm{~cm}$ of lead are placed beyond the condenser ends of the heat pipes, the neutron dose rate drops about 1.5 orders of magnitude. If $19 \mathrm{~cm}$ of borated polyethylene is used, the dose rate drops an additional order of magnitude, but still remains far above appropriate limits. Further analysis is needed with different shield geometries and shield materials to mitigate this exceptionally large dose risk. 


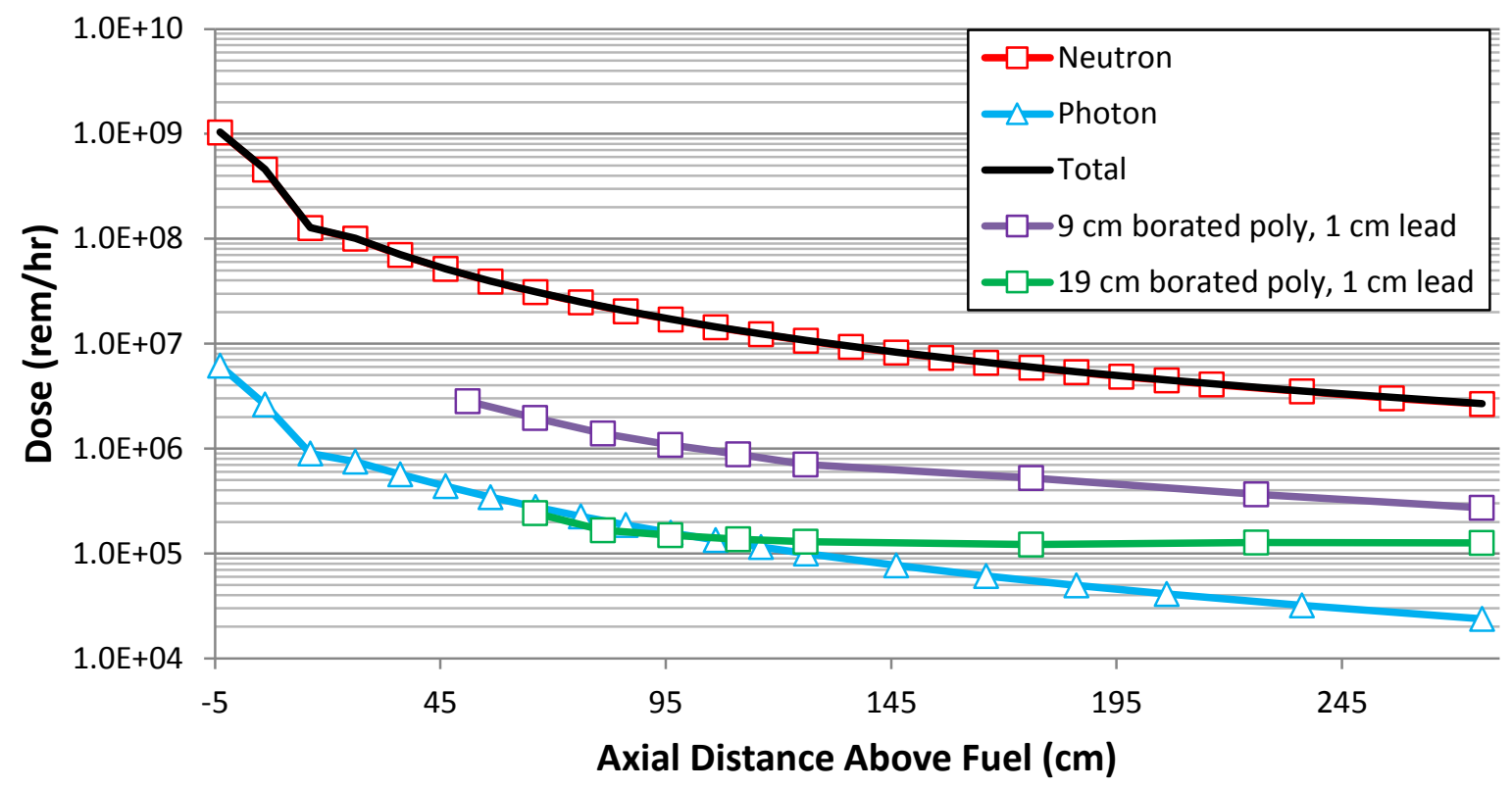

Figure 15. Neutron and photon dose rates above the core.

\section{Parametric and Sensitivity Analyses}

A variety of parametric studies were performed to observe the core reactivity response. For each study, the goal was to maintain the nominal Design A beginning-of-life initial core excess reactivity $\left(k_{e f f}=\right.$ 1.02825). The first design study analyzed the filling of the central void region with additional fuel elements $(\mathrm{FE})^{6}$. With the addition of fuel elements to this center region the active core region could potentially be made smaller (fewer fuel elements) for the same beginning-of-life excess reactivity. However, this in turn means that to maintain $5 \mathrm{MWt}$, each HP will have to remove a greater heat load, and some thermal parameters will increase accordingly.

Filling the central channel allows for the addition of 55 FEs and increases the core reactivity so much so that there is no longer a sufficient shutdown margin (Table 10). Therefore, fuel elements on the core periphery were progressively removed in order to lower the reactivity. As the active core shrinks the reflector thickness can either 1) increase, by 'filling' the space where the outer fuel elements were or 2) remain constant, and thus the total core size can also decrease. The case where the side reflector thickness increases and the number of fuel elements decreases from 1134 to 703 is shown in Figure 16. Table 10 lists the calculated core k-effective for both cases. Not only does the second case have a smaller reflector radius, but also the control drums are moved inward which is why the observed reactivity becomes much less.

Another way to lower the reactivity is to lower the U-235 enrichment. Figure 17 shows the core keffective as a function of enrichment for the core loading of 1189 FEs. This case is for the nominal Design A core with 1134 FEs plus an additional 55 filling the central channel while keeping the side reflector constant. A consistent BOL excess core reactivity (\$3.92) can be obtained with an enrichment of approximately $18.3 \%$, a relatively small decrease in enrichment from the nominal $19.75 \mathrm{wt} \%$.

\footnotetext{
${ }^{6}$ Although the two emergency shutdown rods would no longer be applicable, each control drum pair is designed to be independent from one another, providing plenty of redundancy in shutdown situations.
} 


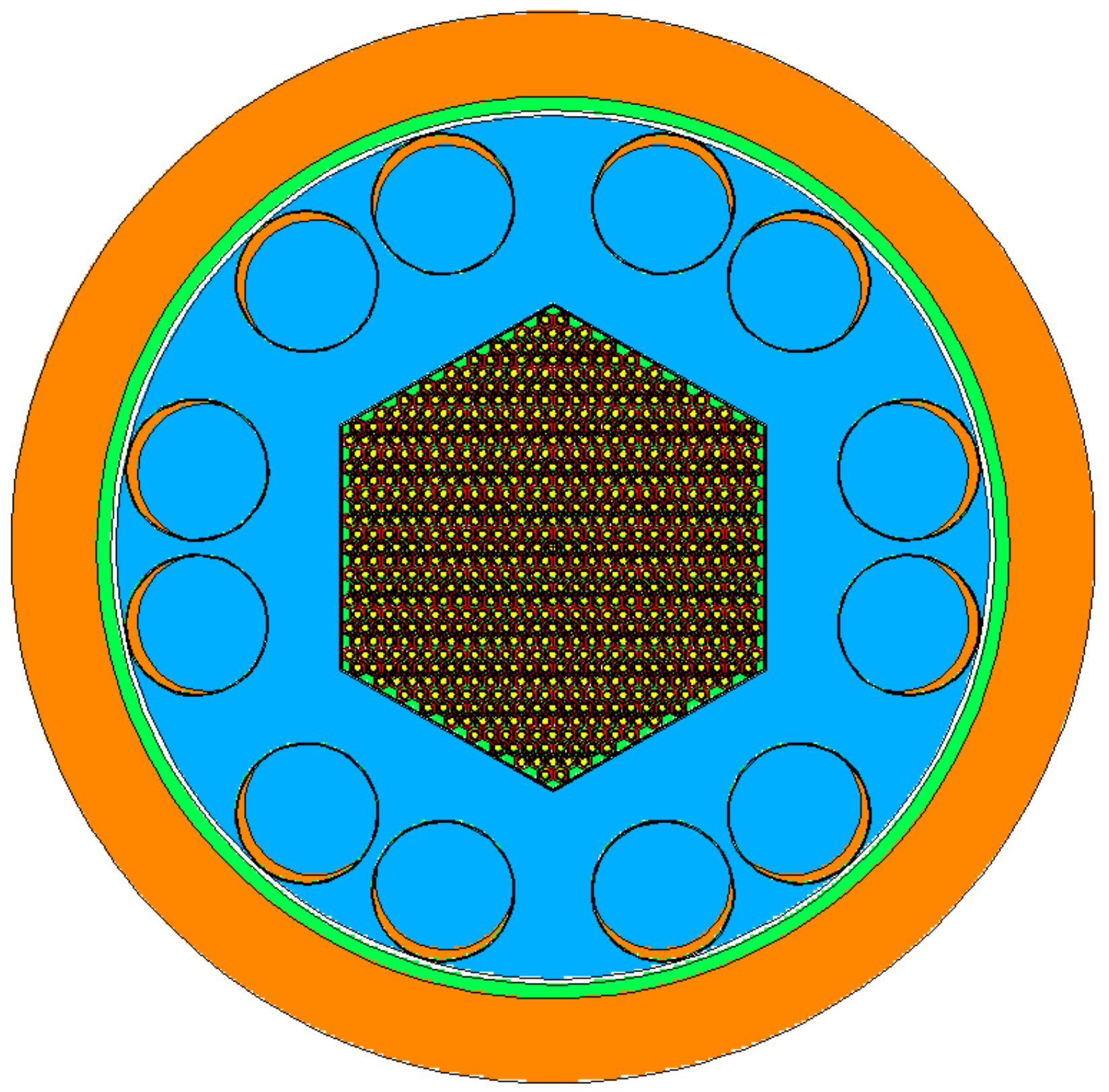

Figure 16. Design A: filling the central void with additional fuel elements and increasing the side reflector thickness. This active core now contains 703 fuel elements total instead of 1,134 in the nominal Design A.

Table 10. Reduce the outer FEs/HPs to lower the core reactivity.

\begin{tabular}{|c|c|c|c|c|c|}
\hline $\begin{array}{l}\text { \# Fuel } \\
\text { Elements / }\end{array}$ & \multirow{2}{*}{$\begin{array}{l}\mathrm{k}-235 \\
\text { Heat Pipes }\end{array}$} & & \multicolumn{4}{|l|}{$\begin{array}{l}\text { Increase Reflector Thickness (control } \\
\text { drum position unchanged) }\end{array}$} & \multicolumn{4}{|l|}{$\begin{array}{l}\text { Maintain Reflector Thickness } \\
(\text { control drums move inward) }\end{array}$} \\
\cline { 3 - 6 } & & \multicolumn{4}{|c|}{$k_{\text {eff }} \pm 0.00002$} \\
\hline & & CDs rotated In & CDs rotated Out & CDs rotated In & CDs rotated Out \\
\hline 1189 & 947 & 0.99950 & 1.06772 & 0.99186 & 1.06505 \\
\hline 1123 & 894 & 0.99820 & 1.06379 & 0.97969 & 1.0571 \\
\hline
\end{tabular}




\begin{tabular}{|c|c|c|c|c|c|}
\hline 1057 & 842 & 0.99606 & 1.05919 & 0.96684 & 1.04848 \\
\hline 991 & 789 & 0.99311 & 1.05361 & 0.9533 & 1.0391 \\
\hline 931 & 742 & 0.98979 & 1.04859 & 0.93916 & 1.02989 \\
\hline 871 & 694 & 0.98583 & 1.04242 & 0.92422 & 1.01988 \\
\hline 811 & 646 & 0.98102 & 1.03492 & 0.90853 & 1.00882 \\
\hline 757 & 603 & 0.97532 & 1.02779 & 0.89067 & 0.99729 \\
\hline 703 & 560 & 0.96962 & 1.01961 & 0.87333 & 0.98554 \\
\hline
\end{tabular}

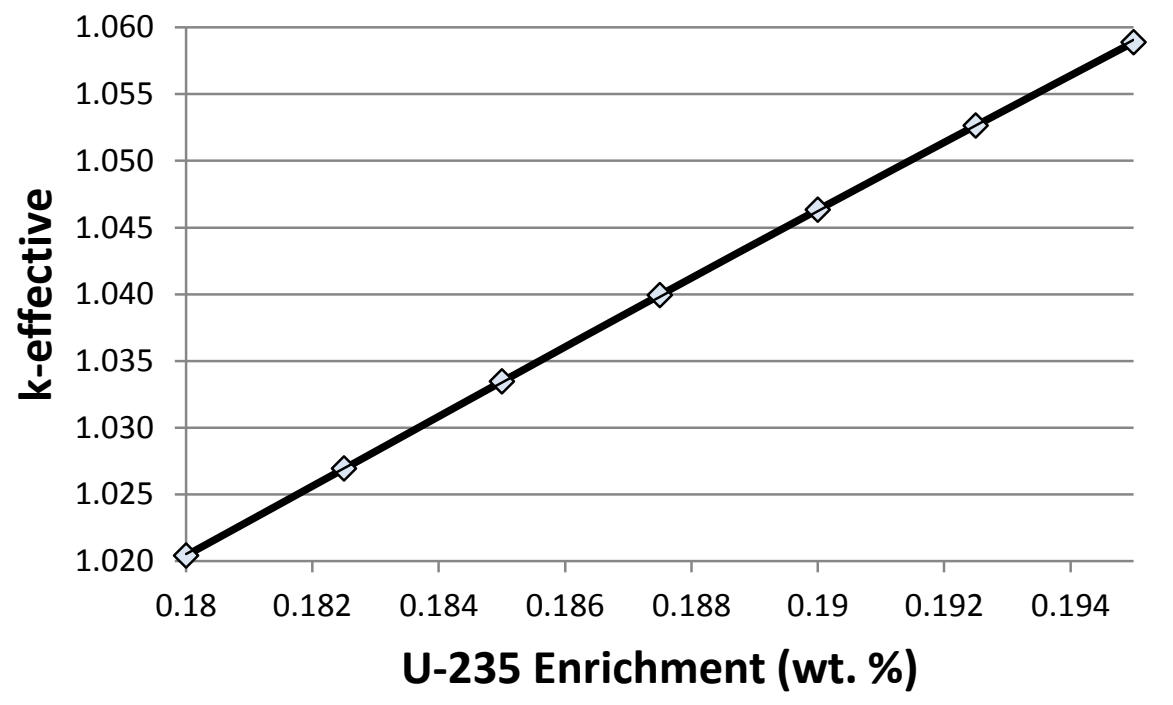

Figure 17. Change in eigenvalue as the fuel enrichment decreases (central cavity filled with fuel).

\section{Heat Pipe Inner Diameter and Pitch}

A second parametric study analyzed the core reactivity as a function of the heat pipe (HP) inner diameter. This is a highly coupled core just like the LANL design, and small changes can have large reactivity impacts. The nominal inner diameter of a HP in Design A is $1.575 \mathrm{~cm}$ (or $0.7875 \mathrm{~cm}$ inner radius). In this parametric study, the HP inner radius was first increased in $0.1 \mathrm{~cm}$ increments up to $1.0875 \mathrm{~cm}$. The fuel pellet cross sectional area per unit cell was held constant, thus the U-235 core mass remains the same. Table 11 lists the resulting new cell dimensions and the associated core k-effective as the HP radius increases. Even with the same amount of U-235 in the core, the reactivity drops nearly 2000 pcm or more with each $0.1 \mathrm{~cm}$ increase in HP radius. This is due to the increase in pitch and hence the core flat-to-flat.

Table 11. Dimensional changes of increasing the heat pipe radius while holding the core fuel mass (fuel pellet cross sectional area) constant.

\begin{tabular}{|c|c|c|c|c|c|c|c|c|c|}
\hline $\begin{array}{c}\text { No. } \\
\text { of } \\
\text { FEs / } \\
\text { HPs }\end{array}$ & $\begin{array}{c}\text { HP Inner } \\
\text { Radius } \\
(\mathrm{cm})\end{array}$ & $\begin{array}{c}\text { Fuel Hex } \\
\text { Apothem } \\
(\mathrm{cm})\end{array}$ & $\begin{array}{c}\text { Pitch } \\
(\mathrm{cm})\end{array}$ & $\begin{array}{c}\text { Flat-to- } \\
\text { Flat } \\
(\mathrm{cm})\end{array}$ & $\begin{array}{c}\text { Fuel Pin Area } \\
\left(\mathrm{cm}^{2}\right)\end{array}$ & $\begin{array}{c}\mathrm{UO}_{2} \\
(\mathrm{~kg})\end{array}$ & $\begin{array}{c}\mathrm{U} \\
(\mathrm{kg})\end{array}$ & $\begin{array}{c}\text { U-235 } \\
(\mathrm{kg})\end{array}$ & k-effective \\
\hline 1134 & 0.7875 & 1.2802 & 2.786 & 99.4 & 2.90 & 5190 & 4573 & 903 & 1.02825 \\
\hline 1134 & 0.8875 & 1.3485 & 2.923 & 102.49 & 2.90 & 5190 & 4573 & 903 & 1.00839 \\
\hline 1134 & 0.9875 & 1.4200 & 3.066 & 107.49 & 2.90 & 5190 & 4573 & 903 & 0.98562 \\
\hline 1134 & 1.0875 & 1.5005 & 3.214 & 112.68 & 2.90 & 5190 & 4573 & 903 & 0.96279 \\
\hline
\end{tabular}


Next, the nominal HP radius was increased by 1 and $2 \mathrm{~mm}$ with the fuel element pitch held constant. This way the unit cell flat-to-flat remains the same and the overall core footprint remains unchanged. Also, keeping the inner and outer cladding thicknesses constant, the increase in the HP radius comes at the expense of the fuel meat. Table 12 lists the resulting new unit cell dimensions and the associated fuel loading as the HP inner radius increases. A $1 \mathrm{~mm}$ increase in the HP radius leads to a reduction of nearly $200 \mathrm{~kg}$ of ${ }^{235} \mathrm{U}$ in the core and greatly reduces the core reactivity. In order to compensate for this, additional fuel elements (FEs) were added to the periphery of the core. Holding the thicknesses of the alumina reflector, $\mathrm{SS}$ core barrel, and $\mathrm{B}_{4} \mathrm{C}$ shield constant, while adding additional peripheral fuel elements, forced these components out radially making the core bigger.

Table 12 lists the resulting reactivity change. It is clear that the cores would have to be much larger. Since a similar excess reactivity was not obtained at the core sizes analyzed, the results were extrapolated to get a rough estimate of the amount of FEs needed. For the increased heat pipe radii of 0.8875 and $0.9875 \mathrm{~cm}$, the number of FEs needed to achieve a core multiplication factor equal to 1.02825 (while keeping the pitch constant) would be roughly 1895 and 4086, respectively. The former would increase the core flat-toflat by nearly $25 \mathrm{~cm}$, and the latter would increase the core upwards of $80 \mathrm{~cm}$. If the central channel were also to be filled with fuel, the number of FEs needed would be somewhat lower at 1823 and 3105 , increasing the flat-to-flat dimension by roughly $19 \mathrm{~cm}$ and $63 \mathrm{~cm}$ respectively, still resulting in a significant increase in core size. Since the reactor is proposed to be readily transportable to remote locations, these larger core sizes may impact the transportability of the reactor.

Table 12. Increase the HP radius (constant pitch) and add additional FEs/HPs to the core.

\begin{tabular}{|c|c|c|c|c|}
\hline $\begin{array}{c}\text { No. of } \\
\text { FEs/HPs }\end{array}$ & $\begin{array}{c}\text { HP Inner Radius } \\
(\mathrm{cm})\end{array}$ & $\begin{array}{c}\text { Core flat-to- } \\
\text { flat }(\mathrm{cm})\end{array}$ & $\begin{array}{c}\text { U-235 } \\
(\mathrm{kg})\end{array}$ & $k_{\text {eff }}$ \\
\hline 1134 & 0.7875 & 99.4 & 903 & 1.02825 \\
\hline 1134 & 0.8875 & 99.4 & 709 & 0.93479 \\
\hline 1278 & 0.8875 & 103.082 & 799 & 0.96183 \\
\hline 1350 & 0.8875 & 105.868 & 845 & 0.97049 \\
\hline 1428 & 0.8875 & 108.654 & 893 & 0.97921 \\
\hline 1506 & 0.8875 & 111.44 & 942 & 0.9873 \\
\hline 1584 & 0.8875 & 114.226 & 991 & 0.99483 \\
\hline 1752 & 0.8875 & 119.798 & 1096 & 1.01011 \\
\hline 1836 & 0.8875 & 122.584 & 1149 & 1.01668 \\
\hline 1134 & 0.9875 & 99.4 & 496 & 0.79517 \\
\hline 1428 & 0.9875 & 108.654 & 625 & 0.84220 \\
\hline 1506 & 0.9875 & 111.44 & 659 & 0.85060 \\
\hline 1584 & 0.9875 & 114.226 & 693 & 0.85840 \\
\hline 1836 & 0.9875 & 122.584 & 803 & 0.88147 \\
\hline 2286 & 0.9875 & 133.728 & 1000 & 0.90779 \\
\hline 2700 & 0.9875 & 147.658 & 1181 & 0.93528 \\
\hline
\end{tabular}

\section{Fuel Mass and Pitch}

A second study increased the inner HP diameter along with the fuel element pitch, so as to allow an increase in the core fuel mass and the flat-to-flat dimension of the overall reactor core. An iterative approach was undertaken to find the fuel pellet hex apothem that yields an equivalent k-effective using the nominal number of FEs/HPs (1134). The pitch will also increase, but the results lead to much more realistic and manageable core changes. As seen in Table 13, with all poisons removed the core keffectives differ by less than $40 \mathrm{pcm}$ compared to the nominal case, and there is plenty of reactivity control shutdown margin. Increasing the HP radius by $1 \mathrm{~mm}$ only increases the core radially by $5 \mathrm{~cm}$, and 
a $4 \mathrm{~mm}$ HP radius increase only leads to a $25 \mathrm{~cm}$ radial core increase. A higher uranium loading will be required in the core, but the overall increase in reactor volume and mass should not pose an issue as far as ease of transportability. Thus, a larger heat pipe can be accommodated with little design change if needed.

Table 13. Increase both the HP radius and the fuel hex apothem, hence the pitch.

\begin{tabular}{|c|c|c|c|c|c|c|c|c|c|c|}
\hline $\begin{array}{c}\text { No. } \\
\text { of } \\
\text { FEs / } \\
\text { HPs }\end{array}$ & $\begin{array}{c}\text { HP } \\
\text { Inner } \\
\text { Radius } \\
(\mathrm{cm})\end{array}$ & $\begin{array}{c}\text { Adjusted } \\
\text { Fuel Hex } \\
\text { Apothem } \\
(\mathrm{cm})\end{array}$ & $\begin{array}{c}\text { Pitch } \\
(\mathrm{cm})\end{array}$ & $\begin{array}{c}\text { Flat-to- } \\
\text { flat }(\mathrm{cm})\end{array}$ & $\begin{array}{c}\text { U- } \\
235 \\
(\mathrm{~kg})\end{array}$ & $\begin{array}{c}\text { All } \\
\text { Poisons } \\
\text { Out } \\
k_{\text {eff }}\end{array}$ & $\begin{array}{c}\text { All } \\
\text { Poisons } \\
\text { In } \\
k_{\text {eff }}\end{array}$ & $\begin{array}{c}\text { CDs In } \\
k_{\text {eff }}\end{array}$ & $\begin{array}{c}\text { Annular } \\
\text { Rod In } \\
k_{\text {eff }}\end{array}$ & $\begin{array}{c}\text { Solid } \\
\text { Rod In } \\
k_{\text {eff }}\end{array}$ \\
\hline 1134 & 0.7875 & 1.2802 & 2.786 & 99.4 & 903 & 1.02825 & 0.84594 & 0.95042 & 0.94555 & 0.95933 \\
\hline 1134 & 0.8875 & 1.372 & 2.970 & 104.136 & 972 & 1.02865 & 0.84400 & 0.94342 & 0.95119 & 0.96470 \\
\hline 1134 & 0.9875 & 1.467 & 3.160 & 110.786 & 1050 & 1.02817 & 0.85349 & 0.94677 & 0.95568 & 0.96878 \\
\hline 1134 & 1.0875 & 1.564 & 3.354 & 117.576 & 1134 & 1.02877 & 0.86418 & 0.95134 & 0.96105 & 0.97365 \\
\hline 1134 & 1.1875 & 1.661 & 3.548 & 124.366 & 1219 & 1.02861 & 0.87352 & 0.95499 & 0.96521 & 0.97729 \\
\hline
\end{tabular}

\section{Inner and Outer Clad Thickness}

A third parametric study focused on the SS fuel cladding thickness. The nominal inner and outer thicknesses are 0.4 and $1.0 \mathrm{~mm}$, respectively. These may need to be increased in order to reduce thermal stresses in these components. As before, the SS clad is increased at the expense of the fuel, first the inner SS then the outer SS in $0.1 \mathrm{~mm}$ increments (the pitch is constant). Similar trends are observed, and the core k-effective decreases. The core will go subcritical if the inner clad thickness is greater than $0.7 \mathrm{~mm}$ or the outer clad thickness is greater than $1.2 \mathrm{~mm}$. Figures 18 and 19 show that adding one additional layer of fuel around the core for a total of 1206 FEs $(1134+72)$ can easily offset slight increases in the cladding thickness.

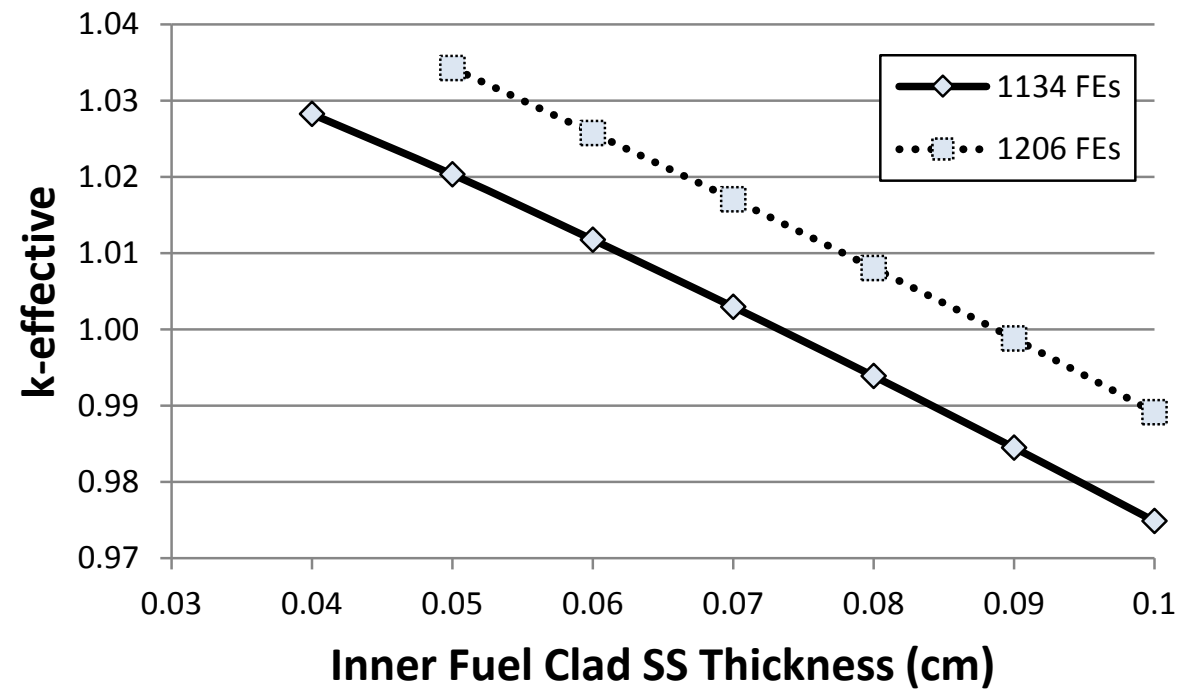

Figure 18. Core k-effective versus inner fuel clad thickness and constant fuel element pitch (fuel meat decrease). 


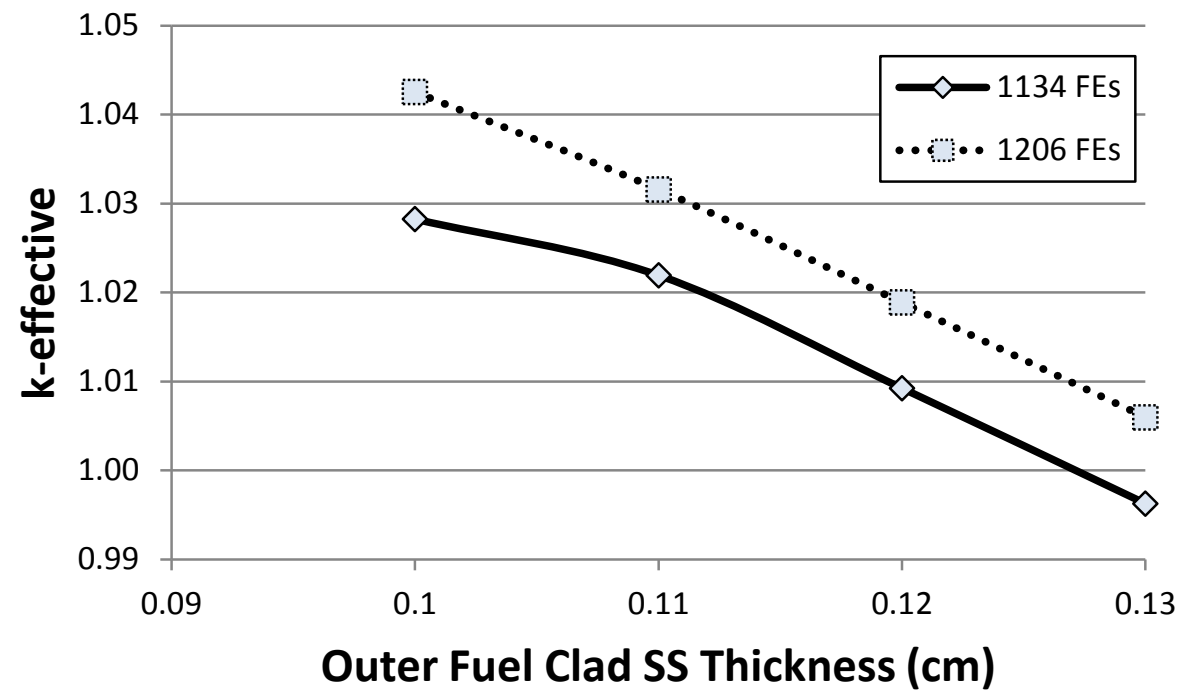

Figure 19. Core k-effective versus outer fuel clad thickness and constant fuel element pitch (fuel meat decrease).

The inner and outer clad were then increased at the same time and with the same thickness. However, the fuel meat thickness was adjusted such that the core has the same k-effective. Again, this was an iterative process that ultimately leads to an increase in the unit cell pitch. This was done for the two cores, nominal case with 1134 elements and the other with 1206 elements (one extra peripheral ring of fuel elements). All k-effectives lie within $+/-45 \mathrm{pcm}$ from the nominal core. Even when both the inner and outer cladding thicknesses are $0.14 \mathrm{~cm}$, the core flat-to-flat only increases by about $10 \mathrm{~cm}$. Additional uranium is needed, but again the increase in core volume and mass does not appear to be an issue.

Table 14. Increase both the inner and outer fuel clad and the fuel meat to maintain the nominal excess reactivity.

\begin{tabular}{|c|c|c|c|c|c|c|c|}
\hline $\begin{array}{c}\text { No. of } \\
\text { FEs / HPs }\end{array}$ & $\begin{array}{c}\text { Inner/Outer Fuel } \\
\text { Clad Thickness } \\
(\mathrm{cm})\end{array}$ & $\begin{array}{c}\text { Adjusted } \\
\text { Fuel Hex } \\
\text { Apothem }(\mathrm{cm})\end{array}$ & Pitch $(\mathrm{cm})$ & $\begin{array}{c}\text { Flat-to-Flat } \\
(\mathrm{cm})\end{array}$ & $\begin{array}{c}\text { Fuel } \\
\text { Pellet } \\
\text { Area } \\
\left(\mathrm{cm}^{2}\right)\end{array}$ & $\begin{array}{c}\text { U-235 } \\
(\mathrm{kg})\end{array}$ & $\begin{array}{c}\text { All Poisons } \\
\text { Out } \\
k_{\text {eff }}\end{array}$ \\
\hline 1134 & 0.10 & 1.333 & 2.8916 & 101.406 & 3.0118 & 938 & 1.02796 \\
\hline 1134 & 0.11 & 1.348 & 2.9416 & 103.156 & 3.0880 & 962 & 1.02856 \\
\hline 1134 & 0.12 & 1.363 & 2.9916 & 104.906 & 3.1651 & 986 & 1.02851 \\
\hline 1134 & 0.13 & 1.377 & 3.0396 & 106.586 & 3.2335 & 1007 & 1.02800 \\
\hline 1134 & 0.14 & 1.392 & 3.0896 & 108.336 & 3.3124 & 1032 & 1.02817 \\
\hline 1206 & 0.10 & 1.322 & 2.8696 & 103.506 & 2.9107 & 964 & 1.02869 \\
\hline 1206 & 0.11 & 1.337 & 2.9196 & 105.306 & 2.9857 & 989 & 1.02849 \\
\hline 1206 & 0.12 & 1.352 & 2.9696 & 107.106 & 3.0616 & 1014 & 1.02893 \\
\hline 1206 & 0.13 & 1.366 & 3.0176 & 108.834 & 3.1290 & 1037 & 1.02826 \\
\hline 1206 & 0.14 & 1.380 & 3.0656 & 110.562 & 3.1971 & 1059 & 1.02794 \\
\hline
\end{tabular}

\section{$\mathrm{UO}_{2}$ Pellet Rounding}

Aside from the cladding thickness, possible thermal stresses may require that the inner wall of the outer SS clad corners be rounded. The rounding of the $\mathrm{UO}_{2}$ pellet was approximated as shown in Figure 20. The $\mathrm{UO}_{2}$ corners are replaced by SS in the outer clad, and this displacement of $\mathrm{UO}_{2}$ fuel will impact the 
calculated k-effective slightly. Each rounded corner has an approximate triangular section of fuel removed. The base of each of these triangles was adjusted from $0.13 \mathrm{~cm}$ up to $0.57 \mathrm{~cm}$ to gauge the sensitivity. Thus the fuel pin area will vary from the nominal value of $2.90 \mathrm{~cm}^{2}$ down to $2.66 \mathrm{~cm}^{2}$. This was examined with the core having the nominal 1134 elements and one with the addition of one extra ring, or 1206 elements; other dimensions are unchanged. Figure 21 shows how the core k-effective behaves as the U-235 content is decreased. Initially, rounding the edges has only a slight negative reactivity effect; the one extra ring of fuel around the core can easily mitigate this. Rounding does not seem to be a significant problem; and having rounded edges along with thicker fuel cladding could simply be accounted for and adjusted through the pitch and a slight increase in the $\mathrm{UO}_{2}$ loading.

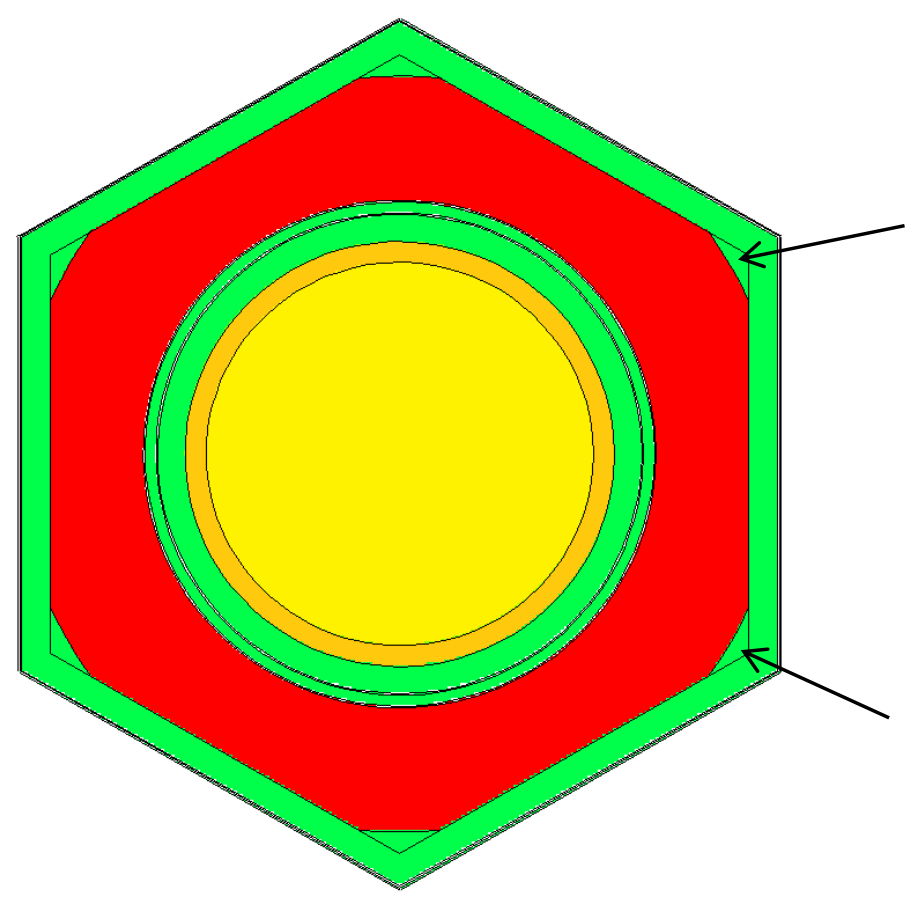

Figure 20. Design A fuel element with rounded inner surface of the outer fuel clad and corresponding rounding of the $\mathrm{UO}_{2}$ pellet corners; arrows show the $\mathrm{UO}_{2}$ space filled by the outer stainless steel clad. 


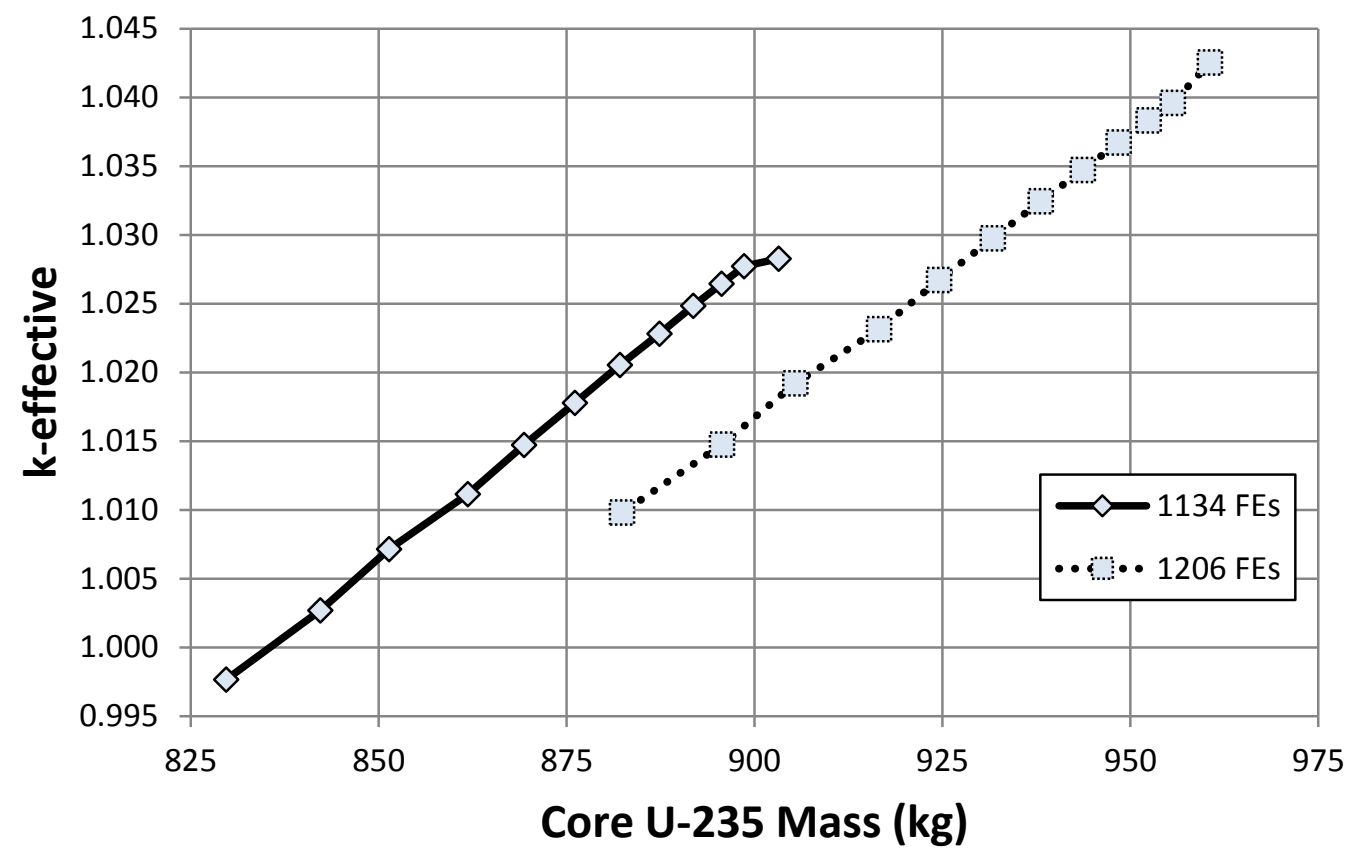

Figure 21. Core k-effective sensitivity to rounding off the $\mathrm{UO}_{2}$ fuel pellet corners (replaced with $\mathrm{SS}$ in outer clad).

\section{UO $_{2}$ Oxide Fuel versus U-10Zr Metallic Fuel}

The last sensitivity study performed on the Design A core involved replacing the $\mathrm{UO}_{2}$ oxide fuel with $\mathrm{U}$ $10 \mathrm{Zr}$ metallic fuel. Although $\mathrm{UO}_{2}$ is the most qualified reactor fuel with a high technology readiness level, U-10Zr fuel has been successfully used in fast spectrum reactors such as the Experimental Breeder Reactors at INL. And due to the higher density theoretical density of $\mathrm{U}-10 \mathrm{Zr}$ at $16.0 \mathrm{~g} / \mathrm{cm}^{3}$ versus 10.96 $\mathrm{g} / \mathrm{cm}^{3}$ for UO2, there will be a much higher U-235 loading for similar enrichments. In fact, directly replacing the $\mathrm{UO}_{2}$ leads to around a $15500 \mathrm{pcm}$ reactivity increase. Two parametric studies were considered here.

The first parametric study looked the progressive removal of outer peripheral fuel elements to reduce the core reactivity considering two additional conditions, (1) the side reflector thickness increases and the control drums remain in their nominal positions, or (2) the side reflector thickness remains constant and the control drums shift inward. These results are shown in Table 15 for a $\mathrm{U}-10 \mathrm{Zr}$ density of $14.5 \mathrm{~g} / \mathrm{cm}^{3}$ or approximately $9.1 \%$ assume porosity. If the reflector thickness remains constant and the control drums move inward, the k-effective decreases as expected.

However, a very interesting phenomenon is observed if the control drums remain in their nominal positions. The k-effective actually increases when the number of FEs drops from 1134 to 1068. This results because 1) when the fuel is removed and the core shrinks, the reflector increases and 2) in the nominal core, each of the 6 peripheral core sides has an extra $1 \mathrm{~cm}$ of SS in order to keep the exact core footprint of the LANL design (flat-to-flat $=99.40 \mathrm{~cm}$ ). This extra SS is however removed during this parametric study when the core becomes smaller or larger. It appears that this extra SS is responsible for large amounts of parasitic neutron absorption and the core should be adjusted accordingly. As can be seen in the last column, the average pin power greatly increases as fuel elements are removed from the core. Again, the thermal limits need further investigation. 
Table 15. Core k-effectives using U-10Zr metallic fuel with different numbers of fuel elements in the core.

\begin{tabular}{|c|c|c|c|c|c|c|}
\hline \multirow{2}{*}{$\begin{array}{c}\text { No. of } \\
\text { FEs/HPs }\end{array}$} & \multirow{2}{*}{$\begin{array}{c}\mathrm{U} 10 \mathrm{Zr} \\
(\mathrm{kg})\end{array}$} & \multirow{2}{*}{$\begin{array}{c}\mathrm{U} \\
(\mathrm{kg})\end{array}$} & \multirow{2}{*}{$\begin{array}{c}\mathrm{U}-235 \\
(\mathrm{~kg})\end{array}$} & $\begin{array}{l}\text { Increase Reflector } \\
\text { Thickness (control drum } \\
\text { position unchanged) }\end{array}$ & $\begin{array}{l}\text { Maintain Reflector } \\
\text { Thickness (control } \\
\text { drums move inward) }\end{array}$ & \multirow{2}{*}{$\begin{array}{c}\text { Average } \\
\text { Pin Power } \\
(\mathrm{kW})\end{array}$} \\
\hline & & & & \multicolumn{2}{|c|}{$k_{e f f} \pm 0.00002$} & \\
\hline 1134 & 6808 & 6128 & 1210 & 1.11622 & - & 4.41 \\
\hline 1068 & 6412 & 5771 & 1140 & 1.11655 & 1.10887 & 4.68 \\
\hline 1002 & 6016 & 5414 & 1069 & 1.10645 & 1.09952 & 4.99 \\
\hline 936 & 5620 & 5058 & 999 & 1.09969 & 1.08433 & 5.34 \\
\hline 876 & 5259 & 4733 & 935 & 1.09135 & 1.07186 & 5.71 \\
\hline 816 & 4899 & 4409 & 871 & 1.08268 & 1.05861 & 6.13 \\
\hline 756 & 4539 & 4085 & 807 & 1.07218 & 1.04414 & 6.61 \\
\hline 702 & 4215 & 3793 & 749 & 1.06289 & 1.03004 & 7.12 \\
\hline
\end{tabular}

The second parametric study simply reduced the U-235 enrichment to match beginning-of-life excess reactivity with the nominal $\mathrm{UO}_{2}$ cases. Figure 22 shows the core k-effective as the U-235 enrichment decreases assuming a $16.0 \mathrm{~g} / \mathrm{cm}^{3}$ density for the U-10Zr. An equivalent beginning-of-life excess reactivity is obtained with the nominal 1134 elements if the U-10Zr enrichment is reduced to $<15.0 \mathrm{wt} \% \mathrm{U}-235$. The U-10Zr therefore affords approximately a $25 \%$ decrease in enrichment, a significant decrease, a significant decrease from $19.75 \mathrm{wt} \% \mathrm{U}-235$.

If the $\mathrm{U}-10 \mathrm{Zr}$ density is assumed to be $14.4 \mathrm{~g} / \mathrm{cm} 3$ (10\% porosity), the $\mathrm{U}-235$ enrichment can be reduced to approximately $16 \mathrm{wt} \% \mathrm{U}-235$, again a significant $20 \%$ decrease in enrichment. The actual porosity needed for the Special Purpose Reactor will probably be between 0 and 10\% porosity, and perhaps significantly closer to $0 \%$, because of the low burnup of the fuel over 5 years.

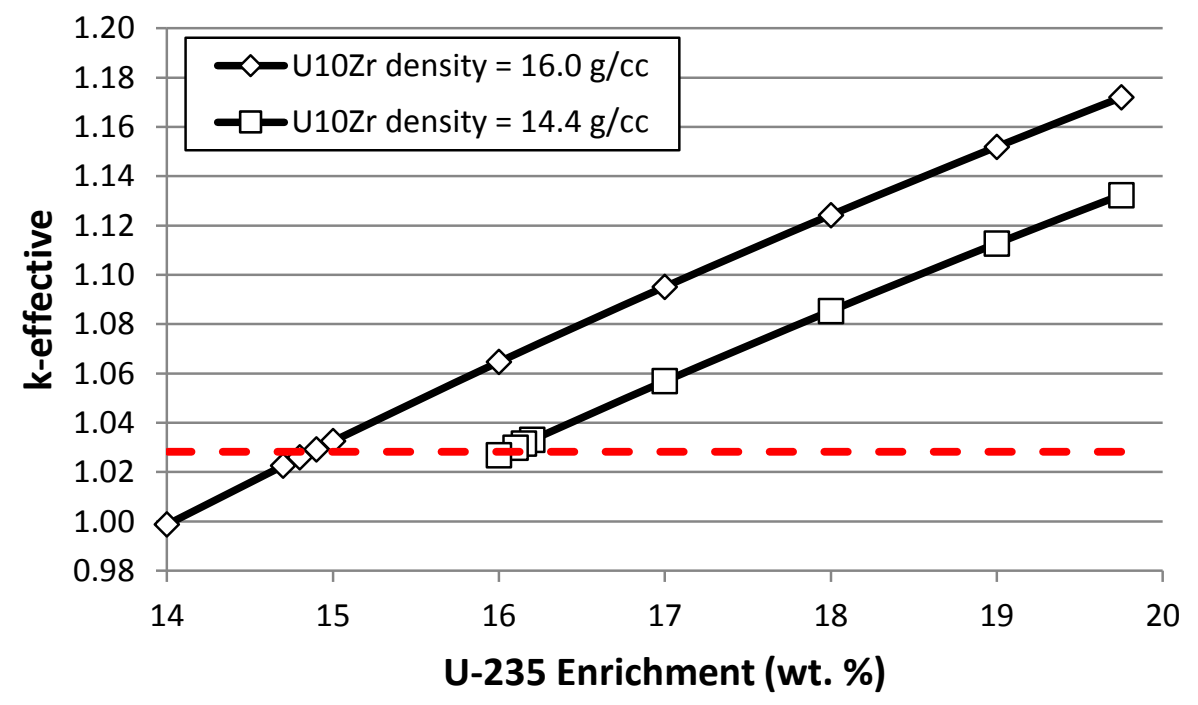

Figure 22. Design A core k-effective versus U-235 enrichment with U-10Zr metallic fuel. 


\section{References}

[1] J. W. Sterbentz, J. E. Werner, M. G. McKellar, A. J. Hummel, J. C. Kennedy, R. N. Wright, J. M. Biersdorf, "Special Purpose Nuclear Reactor (5MW) for Reliable Power at Remote Sites Assessment Report - Using Phenomena Identification and Ranking Tables (PIRTs)," INL/EXT-16-40741, April 2017. [2] P. R. Mcclure, D. I. Poston, D. V. Rao, R. S. Reid, "Design of Megawatt Power Level Heat Pipe Reactors," LA-UR-15-28840, November, 2015.

[3] Monte Carlo N-Particle Transport Code System Including MCNP6.1, MCNP5-1.60, MCNPX-2.7.0 and Data Libraries. Initial MCNP6 Release Overview - MCNP6 version 1.0. Los Alamos National Laboratory, Los Alamos, New Mexico, August 2013.

[4] C. T. Ewing, J. P. Stone, J. R. Spann, R. R. Miller, "High Temperature Properties of Potassium," NRL Report 6233, Inorganic and Nuclear Chemistry Branch, Chemistry Division, September 24, 1965. [5] V. Sobolev, "Database of thermophysical properties of liquid metal coolants for GEN-IV," Studiecentrum Voor Kernenergie Centre D’Etude De L'Energie Nucleaire, November 2010.

[6] J. Duderstadt, L. Hamilton, Nuclear Reactor Analysis, John Wiley \& Sons, Inc., 1976.

[7] ORNL, 2009, SCALE: A Modular Code System for Performing Standardized Computer Analyses for Licensing Evaluations, ORNL/TM-2005/39, Version 6, Vols, I-III, Oak Ridge National Laboratory, January 2009.

[8] Simanullang, I. L., Y. Honda, Y. Fukaya, M. Goto, Y. Shimazaki, N. Fujimoto, and S. Takada, 2016, "Calculation of Decay Heat by New ORIGEN Libraries for High Temperature Engineering Test Reactor," Department of HTTR Oarai Research and Development Center Sector of Nuclear Science Research, JAEA-Technology 2015-032, January 2016.

[9] LANL, 2013, MCNP6 User's Manual Version 1.0, LA-CP-13-00634, Rev. 0, May 2013.

[10] 10CFR20.1201, Subpart C-Occupational Dose Limits, https://www.nrc.gov/reading-rm/doccollections/cfr/part020/part020-1201.html 


\section{Appendix B}

\section{Neutronics Analysis -- Design B}

This appendix presents details of a preliminary neutronic analysis for Design B. Included in this appendix are descriptions of the computer codes, computer models, and assumptions used to perform the parametric studies in order to evaluate Design B reactor thermal sensitivities and characteristics to help evaluate and support the overall Design B reactor system. 


\section{Design B}

\section{Core Description}

The active core layout of Design B is very similar to the LANL concept, but rather than have a monolith structure the lattice arrangement, depicted in Figure 23, sits in a liquid sodium pool. The core is likewise divided into 6 symmetrical $60^{\circ}$ sectors and each sector is sealed off with its own sodium. All walls are double plated stainless steel (SS) and 6 SS spacer grid plates hold the lattice structure in place. Each sector contains 352 fuel pins and 204 heat pipes, identical to the LANL design, arranged in a triangular pitch. However, with all elements independently clad and a minimum web thickness limitation on the spacer plates, the lattice pitch has to be increased. This leads to a slight increase in the core footprint (approximately a $12 \mathrm{~cm}$ radial increase) as well as an increase in the fuel radius from $0.706 \mathrm{~cm}$ to 0.746 $\mathrm{cm}$ to maintain the needed core excess reactivity. Each fuel pin is surrounded by 3 heat pipes, and SS plates are used as the upper and lower axial reflectors.

Core dimensions are given in Table 16. There is approximately 110 additional $\mathrm{kg}$ of ${ }^{235} \mathrm{U}$ in this core compared to the LANL design. Also, the LANL design has an air gap dividing the six sectors, but this gap contains SS in Design B. The sodium coolant is expected to be relatively stagnant in this core, but there is a small gap between each element and the SS spacer plates to allow for axial movement. As seen if Figure 24, the core is likewise surrounded by an alumina reflector with 12 rotatable control drums.

Table 16. Design B active core dimensions and parameters.

\begin{tabular}{|l|l|l|l|}
\hline \multicolumn{5}{|c|}{ Design B Lattice Dimensions / Core Parameters } \\
\hline K vapor radius $(\mathrm{cm})$ & 0.71 & Axial SS reflector plates $(\mathrm{cm})$ & 15.0 \\
\hline K liquid radius $(\mathrm{cm})$ & 0.7875 & Pitch $(\mathrm{cm})$ & 1.8 \\
\hline HP SS clad radius $(\mathrm{cm})$ & 0.8875 & Fuel area $\left(\mathrm{cm}^{\wedge} 2\right)$ & 1.75 \\
\hline Coolant gap radius $(\mathrm{cm})$ & 0.894 & Fuel pin height $(\mathrm{cm})$ & 150 \\
\hline Fuel radius $(\mathrm{cm})$ & 0.746 & Fuel pin volume $(\mathrm{cc})$ & 262 \\
\hline Gap radius $(\mathrm{cm})$ & 0.7525 & Inner hex center-to-flat $(\mathrm{cm})$ & 10.65 \\
\hline Fuel clad radius $(\mathrm{cm})$ & 0.7825 & Inner circle radius $(\mathrm{cm})$ & 10.25 \\
\hline Spacer plate coolant gap radius $(\mathrm{cm})$ & 0.7890 & Outer hex center-to-flat $(\mathrm{cm})$ & 55.90 \\
\hline $\begin{array}{l}\text { Core segment inner tank SS wall } \\
\text { thickness }(\mathrm{cm})\end{array}$ & 0.5 & Total $\mathrm{UO}_{2}(\mathrm{~kg})$ & 5828 \\
\hline $\begin{array}{l}\text { Core segment outer tank SS wall } \\
\text { thickness }(\mathrm{cm})\end{array}$ & 0.3 & Total $\mathrm{U}(\mathrm{kg})$ & 5136 \\
\hline SS spacer plate thickness $(\mathrm{cm})$ & 0.5 & Total ${ }^{235} \mathrm{U}(\mathrm{kg})$ & 1014 \\
\hline
\end{tabular}




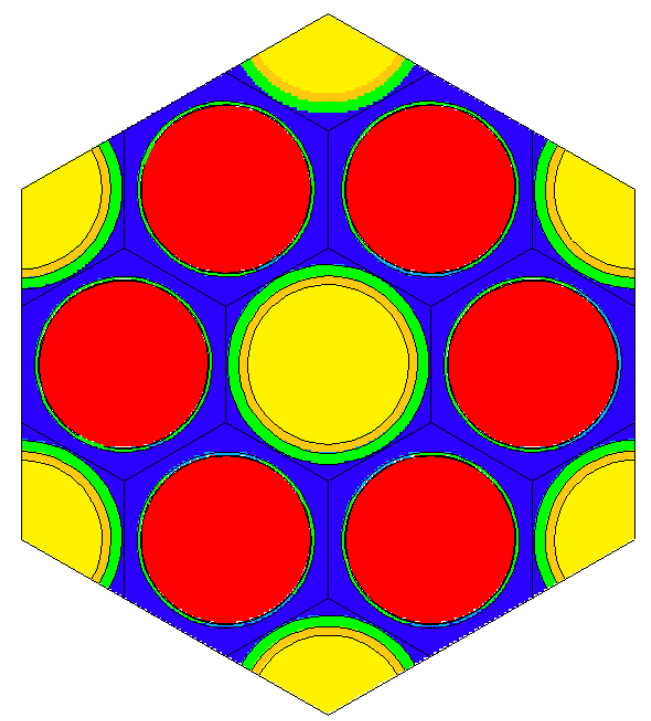

Figure 23. Lattice structure of Design B. Each fuel pin (red) is adjacent to 3 heat pipes (yellow) in a liquid sodium pool (blue).

\section{$\underline{\text { Reactivity Control }}$}

The control drums are worth more in Design B than Design A, but the emergency shutdown rods are worth less. The lower rod worth most likely result due to the fact that the Design B core is roughly $12 \mathrm{~cm}$ larger in diameter than Design A, thus negative reactivity inserted in the center has less of an effect. From a geometric standpoint, the smaller Design A core should have more radial leakage, but at the same time the axial $\mathrm{BeO}$ reflectors in Design A are overwhelmingly more efficient than the SS plates used in Design $\mathrm{B}$ at reflecting neutrons. The different reactivity control parameters are given in Table 17 for Design $\mathrm{B}$ compared to the LANL design. The beginning-of-life core excess reactivity is greater in Design B, but just like the other two cores, there is sufficient shutdown margin with each mechanism.

Table 17. Reactivity control.

\begin{tabular}{|l|l|l|}
\hline & Design B & LANL \\
\hline Core Reactivity Control & \multicolumn{2}{|c|}{$k_{\text {eff }} \pm 0.00002$} \\
\hline All Poisons Out & 1.02417 & 1.02153 \\
\hline All Poisons In & 0.84438 & 0.82500 \\
\hline Control Drums In & 0.93707 & 0.92602 \\
\hline Annular Shutdown Rod In & 0.95107 & 0.94211 \\
\hline Solid Shutdown Rod In & $0.96477 \quad \beta=0.007$ \\
\hline & \multicolumn{2}{|c|}{$\beta$} \\
\hline BOL Excess Reactivity (\$) & 3.37 & 2.88 \\
\hline Total Drum Worth (\$) & 12.97 & 14.42 \\
\hline Individual Drum Worth (\$) & 1.10 & 1.21 \\
\hline Critical Control Drum Rotation $\left(^{\circ}\right)$ & 56 & 48 \\
\hline Annular Shutdown Rod Worth (\$) & 10.72 & 11.79 \\
\hline Solid Shutdown Rod Worth (\$) & 8.59 & 9.58 \\
\hline
\end{tabular}




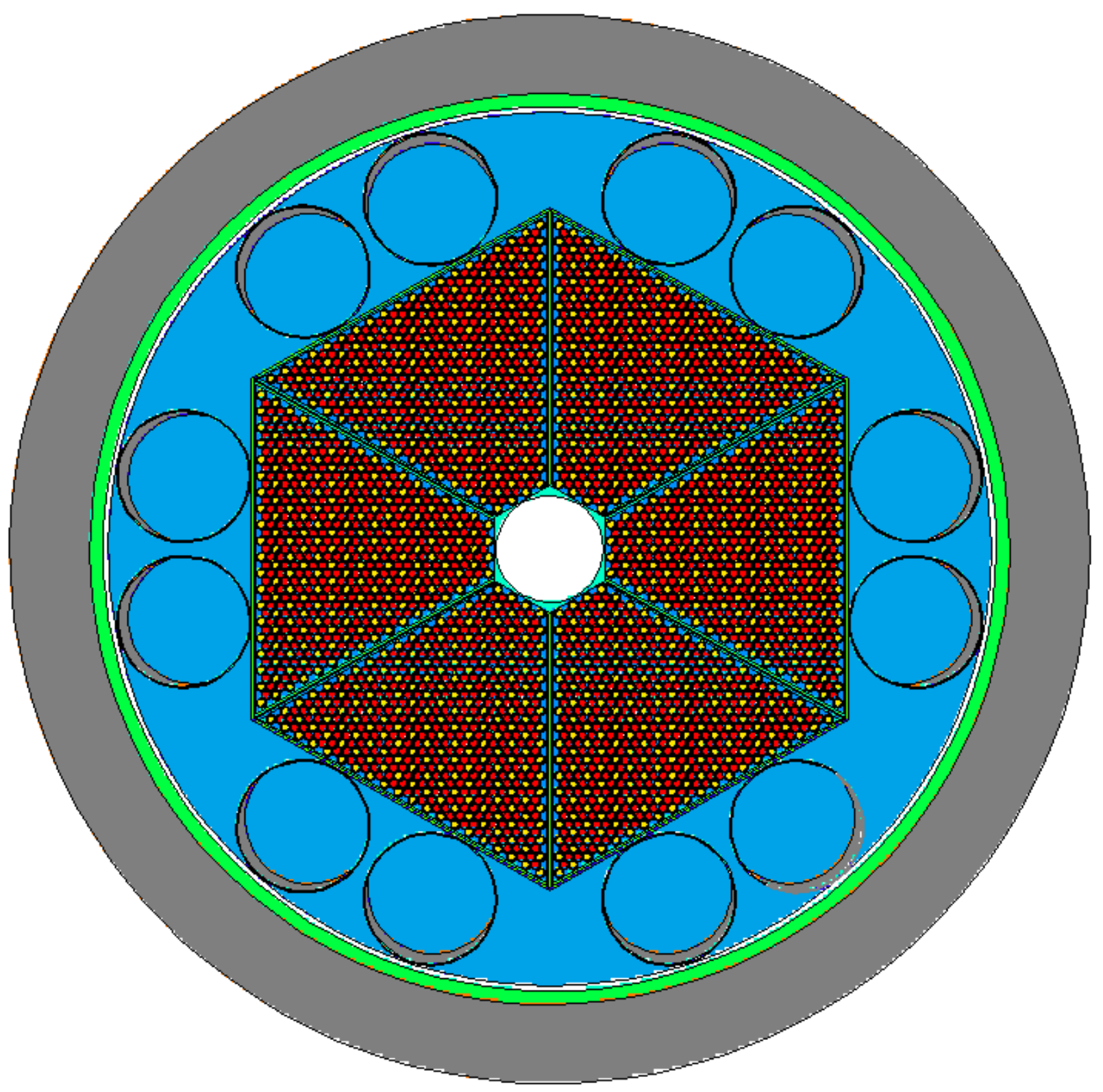

Figure 24. Axial view of the full reactor with the Design B active core.

\section{$\underline{\text { Reactivity Feedback }}$}

Table 18 lists the worth of each feedback mechanism, each of which was calculated independently. The primary reactivity effect again results from the Doppler broadening (Figure 25) of the low-enriched $\mathrm{UO}_{2}$ fuel, amounting to -0.1355 cents $/{ }^{\circ} \mathrm{C}$. This effect is more pronounced in Design $\mathrm{B}$ than the other two cores because there is approximately $560 \mathrm{~kg}$ more uranium. Other negative reactivity feedback effects include (1) thermal expansion of the fuel, (2) alumina reflector radial thermal expansion, and (3) coolant voiding. Because the fuel clad in Design B is only $0.3 \mathrm{~mm}$ thick compared to $1.0 \mathrm{~mm}$ for Design A, it therefore has a much more negligible feedback effect in the former core design. If the cladding is to be increased, then a further analysis should quantify this effect. The fuel expansion was examined in the same manner as before, and Figure 26 shows how the core k-effective decreases when the fuel elongates. Again, because there is more fuel in Design B this effect is slightly more pronounced. The reduction in the alumina radial 
reflector number density will again lead to an increase in radial core leakage (a negative reactivity insertion).

The coolant void coefficient of reactivity seen in Design B results from a decrease in the parasitic absorption in the sodium. Although this is the least in magnitude over the nominal temperature range, Figure 27 shows that if the core were to approach dry-out conditions, the effect becomes much larger. This was calculated by expanding the sodium volume in the axial direction only, assuming that each of the 6 sectors (tanks) has a gap for expansion at the top.

Table 18. Core reactivity feedback coefficients

\begin{tabular}{|c|c|c|}
\hline Feedback Effect (cents $\left./{ }^{\circ} \mathrm{C}\right)$ & Design B & LANL \\
\hline Doppler & -0.1355 & -0.1011 \\
\hline $\mathrm{UO}_{2}$ Fuel Axial Elongation & -0.0462 & -0.0408 \\
\hline Alumina Reflector Radial Thermal Expansion ${ }^{7}$ & -0.0225 & -0.0225 \\
\hline Coolant Void COR & -0.01033 & - \\
\hline SS Monolith Thermal Expansion & - & -0.06034 \\
\hline Total & -0.2145 & -0.2247 \\
\hline
\end{tabular}

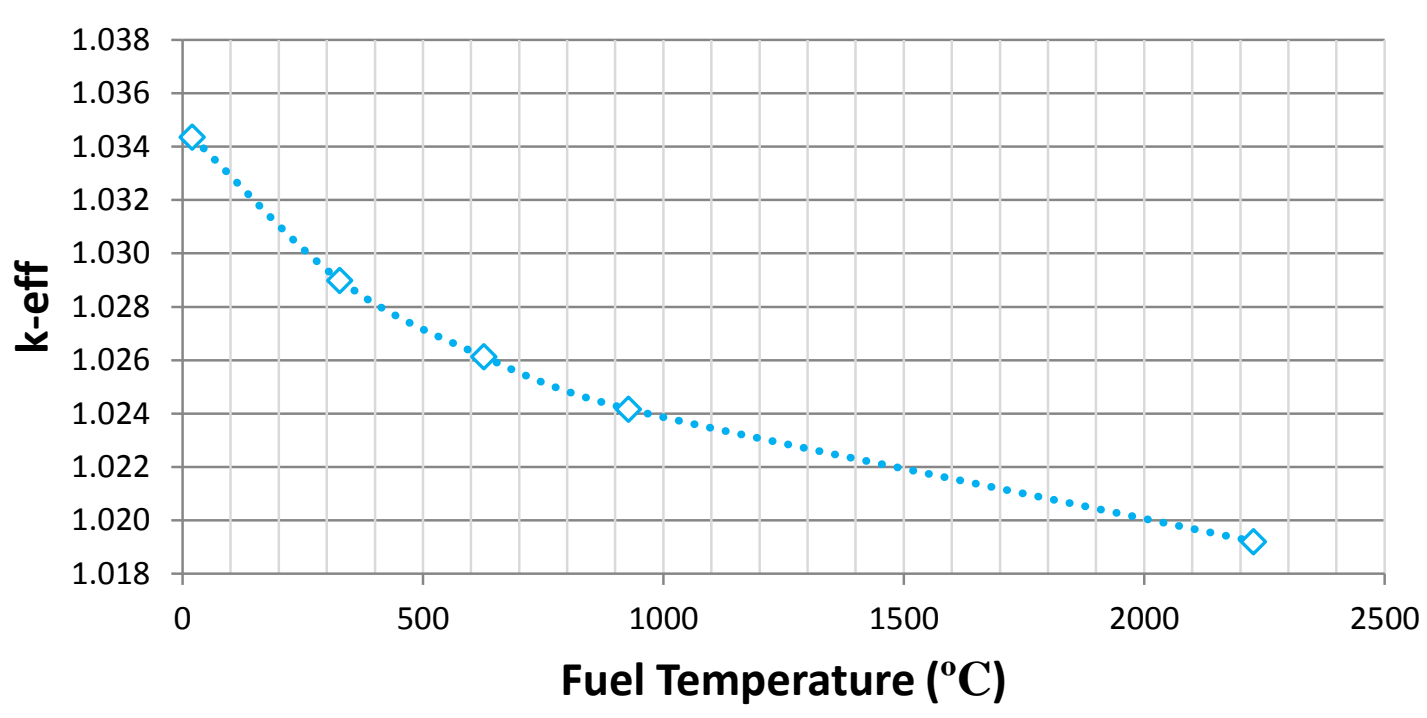

Figure 25. Negative reactivity effect due to U-238 Doppler-broadening in the $\mathrm{UO}_{2}$ fuel.

\footnotetext{
${ }^{7}$ This parameter was not directly calculated for these cores. However, the alumina reflector has the exact same geometry and material properties in both designs and is thus assumed to have a very comparable effect.
} 


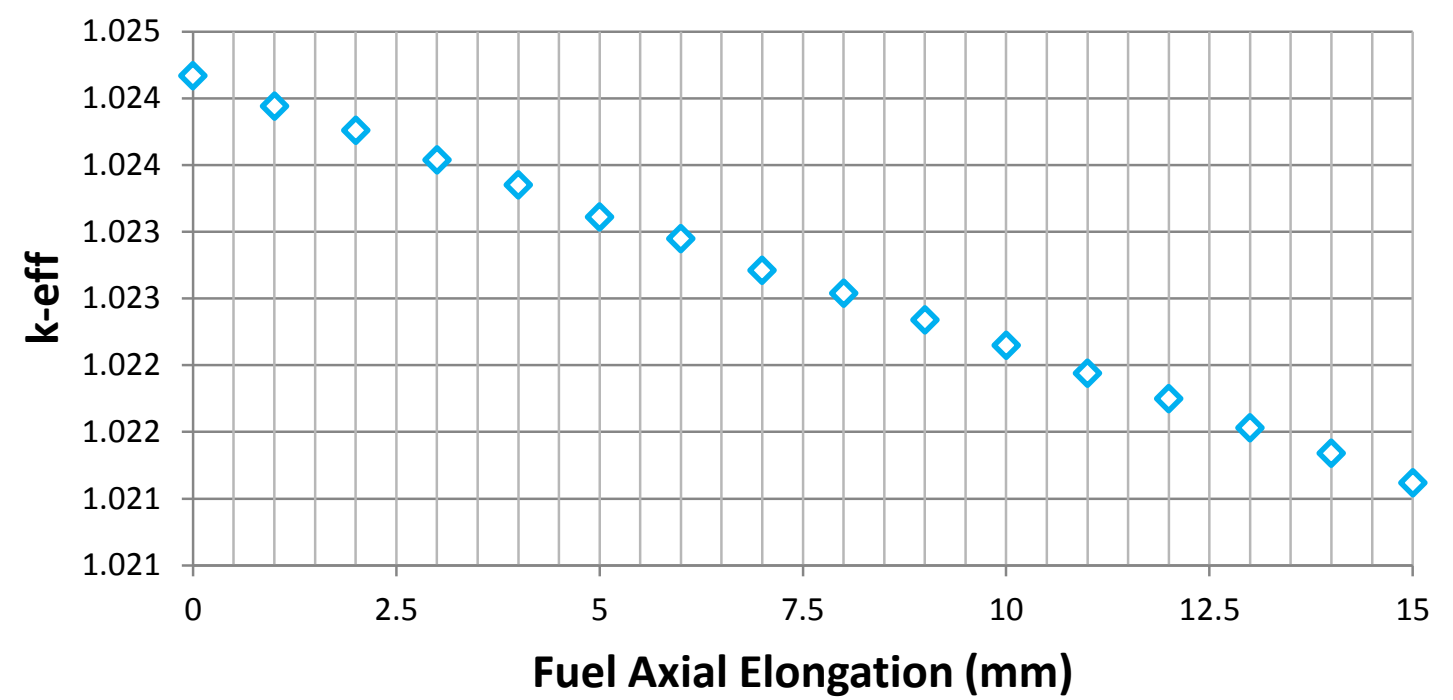

Figure 26. Negative reactivity feedback due to axial elongation of the $\mathrm{UO}_{2}$ fuel.

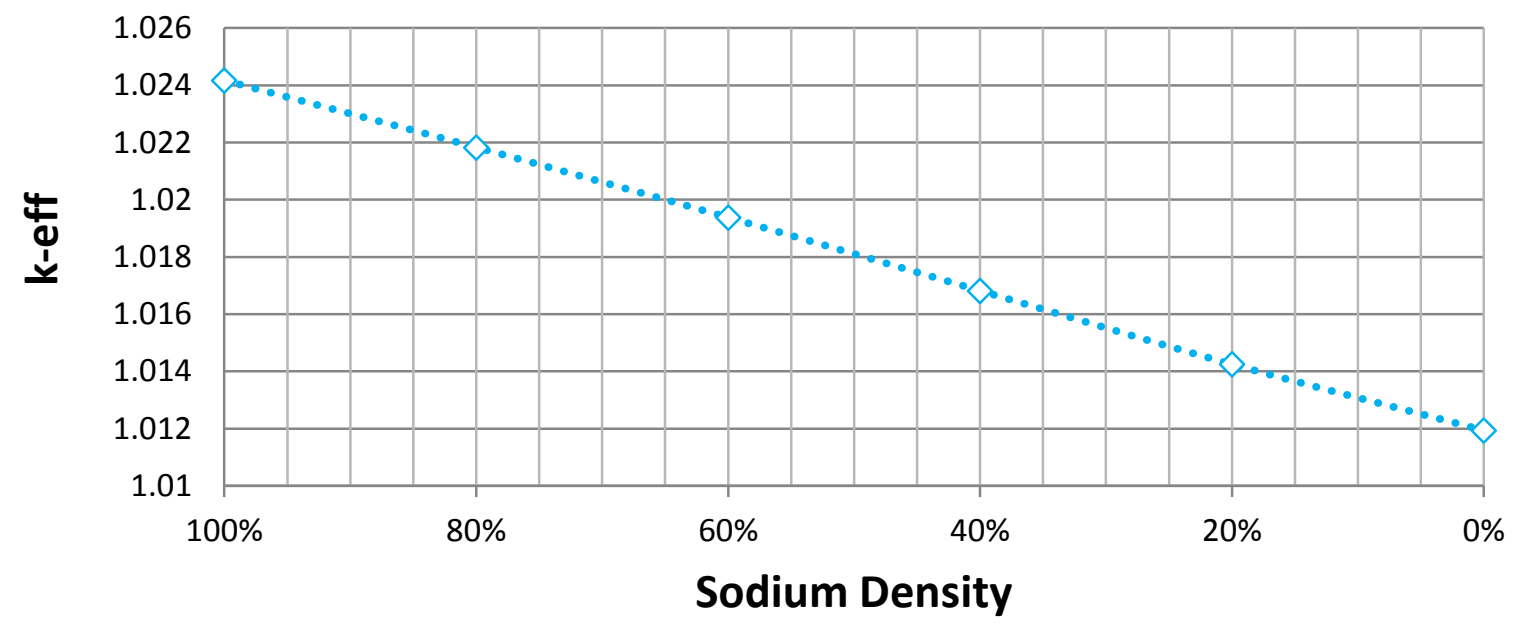

Figure 27. Coolant void coefficient of reactivity (nominal sodium density reduced in $20 \%$ intervals).

\section{Power and Burnup Estimates}

Design B has the same number of fuel pins and heat pipes as the LANL design, thus the average pin power is the same. However, because Design B has a greater pitch (larger active core), the power peaking across the core is greatly reduced (1.50 to 1.20$)$. Also, Design B contains approximately $100 \mathrm{~kg}$ more U235 to compensate for beginning-of-life core excess reactivity. This leads to a roughly $27 \%$ lower peak power density.

Figure 28 shows the peak-to-average pin power for the hottest pin, and Table 19 lists the thermal and burnup parameters. Just like the other designs, there is very little burnup and core reactivity swing in Design B (Figure 29). 


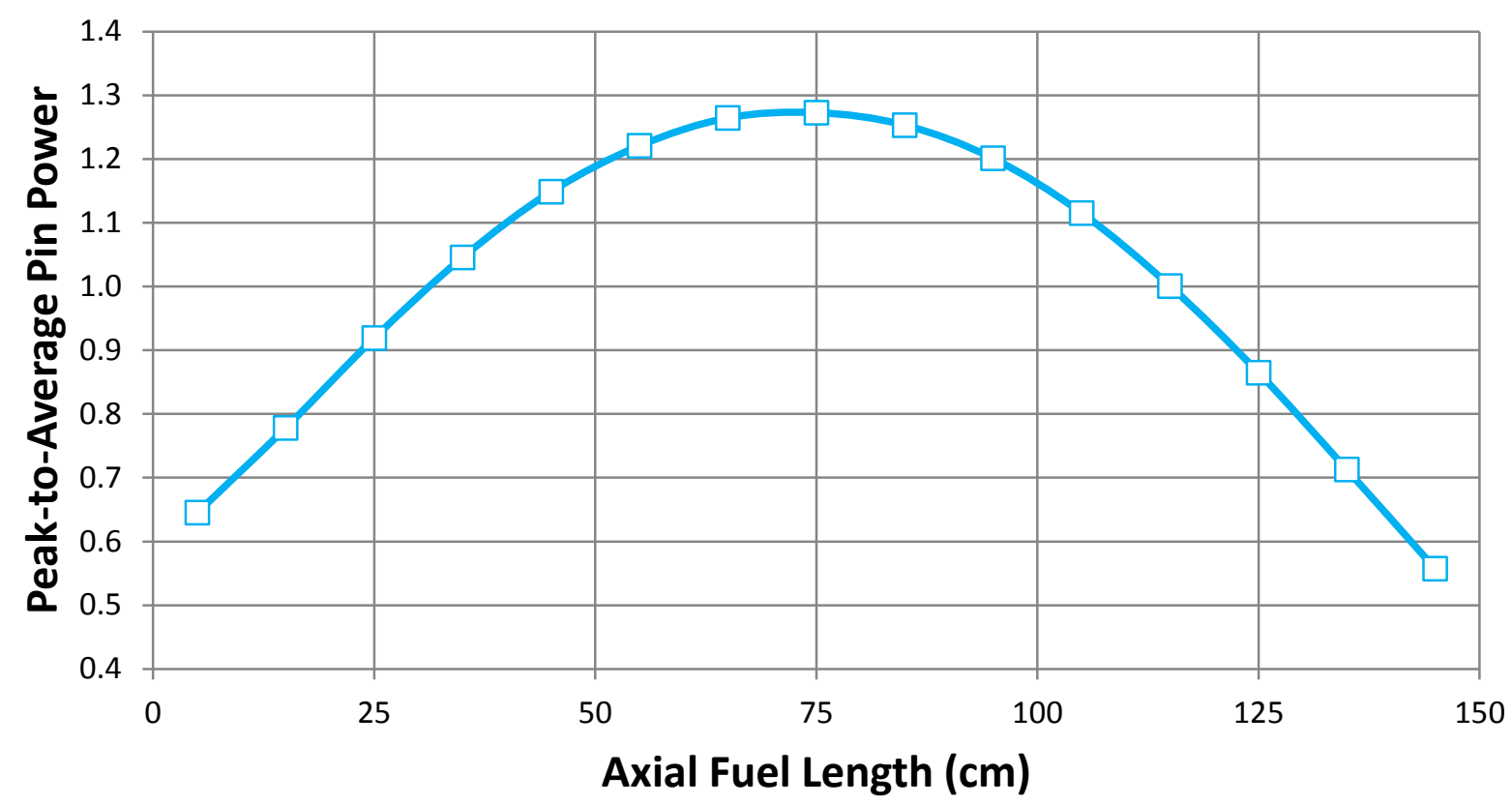

Figure 28. Axial peak-to-average power profile in the hottest pin (Design B).

Table 19. Design B and LANL thermal and core burnup parameters.

\begin{tabular}{|l|l|l|}
\hline & Design B & \multicolumn{1}{|c|}{ LANL $^{8}$} \\
\hline Number of fuel pins & \multicolumn{1}{|c|}{2112} & \multicolumn{1}{|c|}{2112} \\
\hline Average pin power $(\mathrm{kW})$ & 2.37 & 2.37 \\
\hline Max pin power $(\mathrm{kW})$ & 2.84 & 3.55 \\
\hline Peak-to-average & 1.20 & 1.50 \\
\hline Pin peaking factor (axial) & 1.27 & 1.29 \\
\hline Peak linear heat rate $(\mathrm{kW} / \mathrm{m})$ & 1.89 & 2.37 \\
\hline Average power density $\left(\mathrm{W} / \mathrm{cm}^{3}\right)$ & 9.03 & 9.90 \\
\hline Peak power density $\left(\mathrm{W} / \mathrm{cm}^{3}\right)$ & 10.82 & 14.9 \\
\hline $\mathrm{U}$ mass $(\mathrm{kg})$ & 5136 & 4600 \\
\hline U-235 mass $(\mathrm{kg})$ & 1014 & 908 \\
\hline Specific power $(\mathrm{MW} / \mathrm{tHM})$ & 0.9735 & 1.087 \\
\hline Average Burnup $(\mathrm{GWd} / \mathrm{t})$ & 1.8 & 2.0 \\
\hline \%U-235 depletion & 1.11 & 1.0 \\
\hline FIMA (\%) & 0.21 & 0.33 \\
\hline Peak fission density (fissions/cm 3 ) & $4.88 \mathrm{E}+19$ & $7.80 \mathrm{E}+19$ \\
\hline
\end{tabular}

${ }^{8}$ These parameters were calculated according to the INL model of the LANL design and may differ slightly from those reported by LANL. 


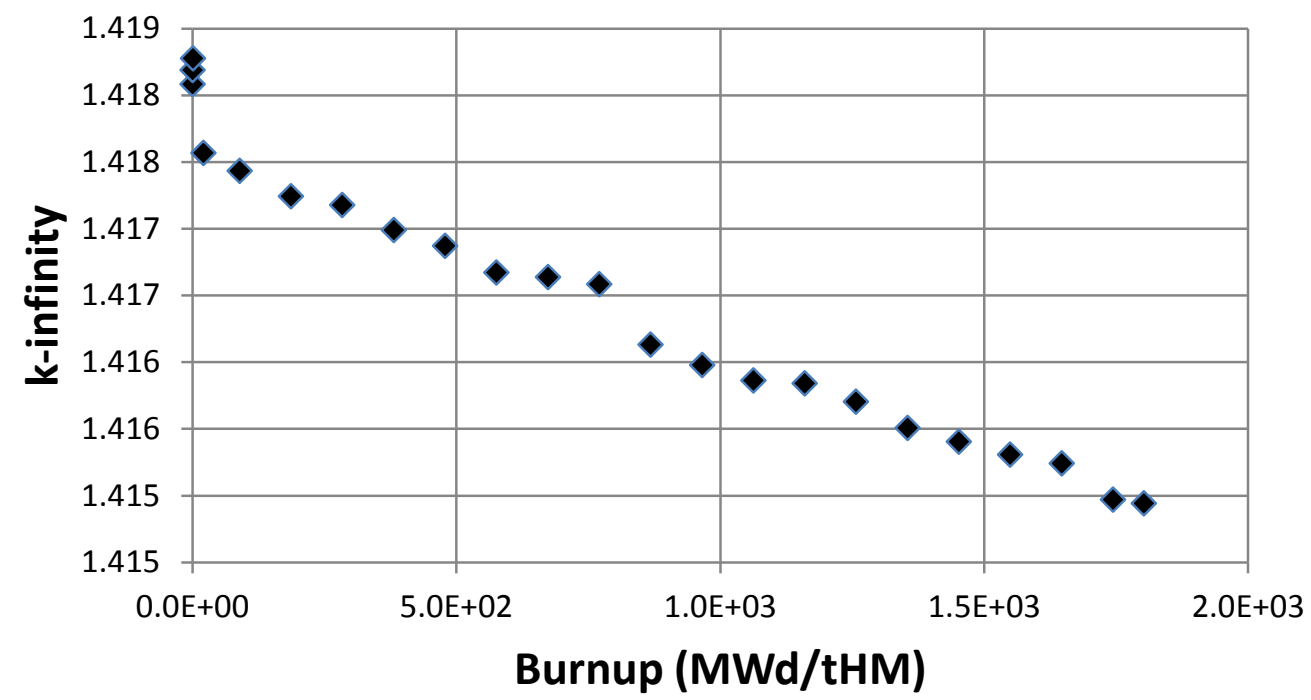

Figure 29. Simplified infinite lattice model depleted over 5 years.

\section{Decay Heat}

Decay heat curves were generated in the same manner as those for Design A. The curves are nearly identical and the trends are the same. The main difference stems from the fact that Designs A \& B have different core specific powers (MW/gram) due to the different amounts of uranium loading. The curves are shown in Figures 30 and the fractional contribution in Figure 31.

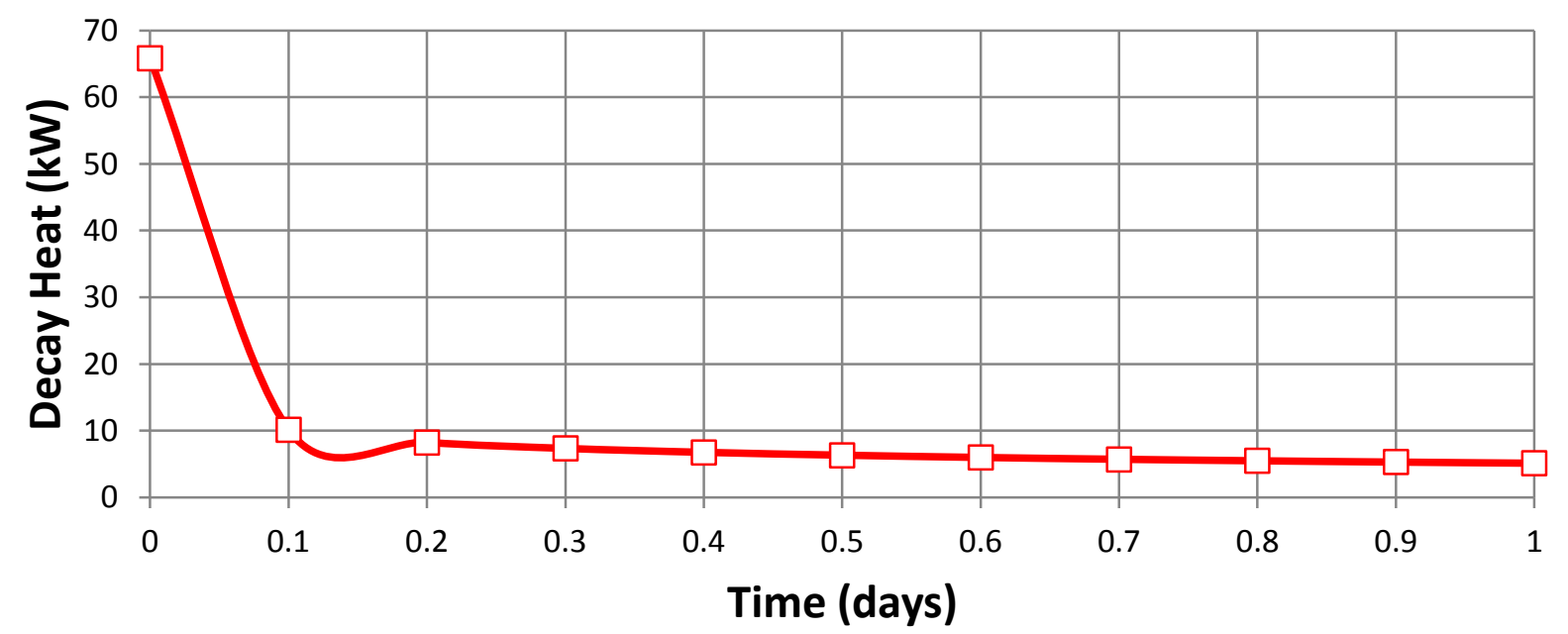

Figure 30. Design B total core decay heat following shutdown. 

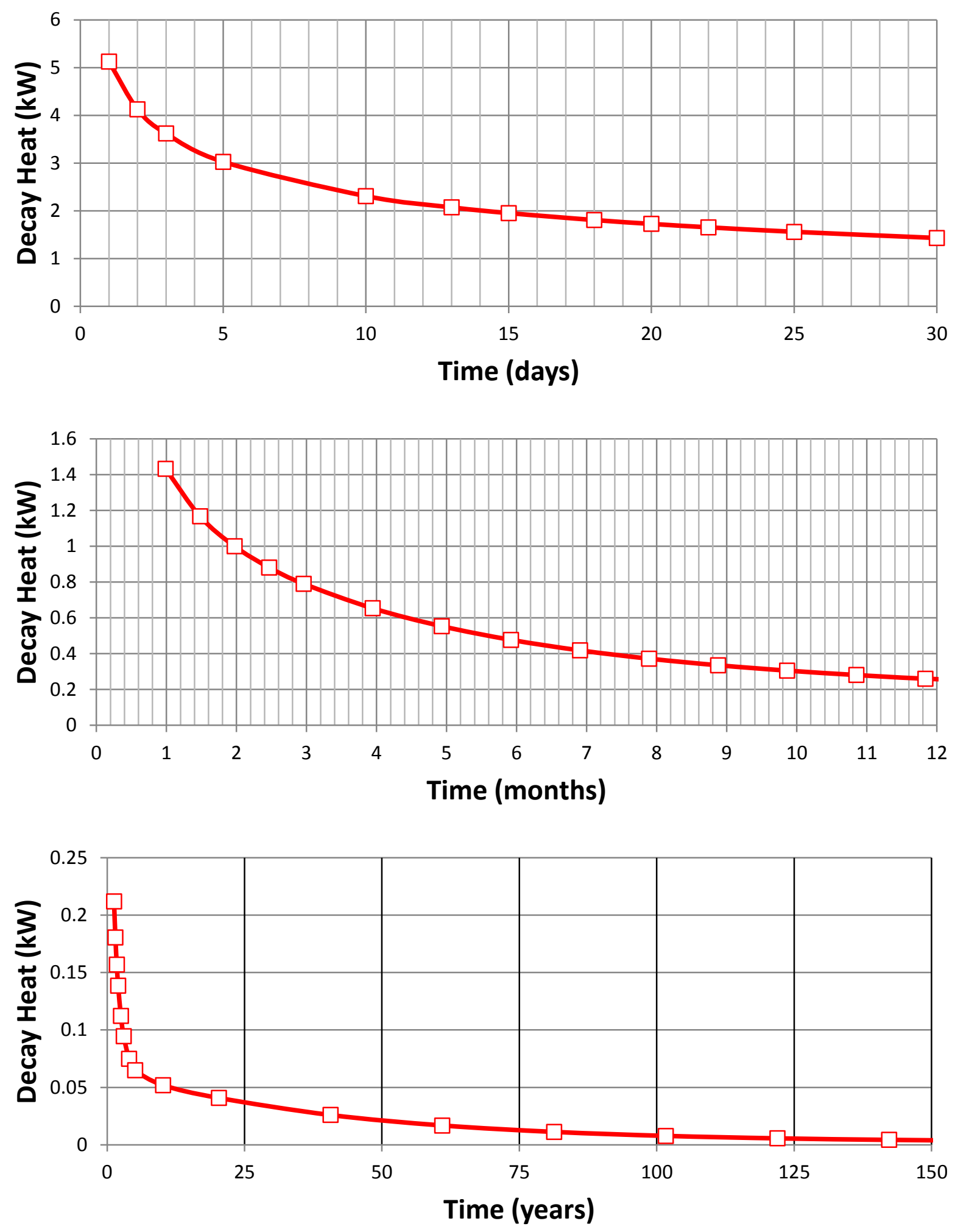

Figure 30. Design B total core decay heat following shutdown. 


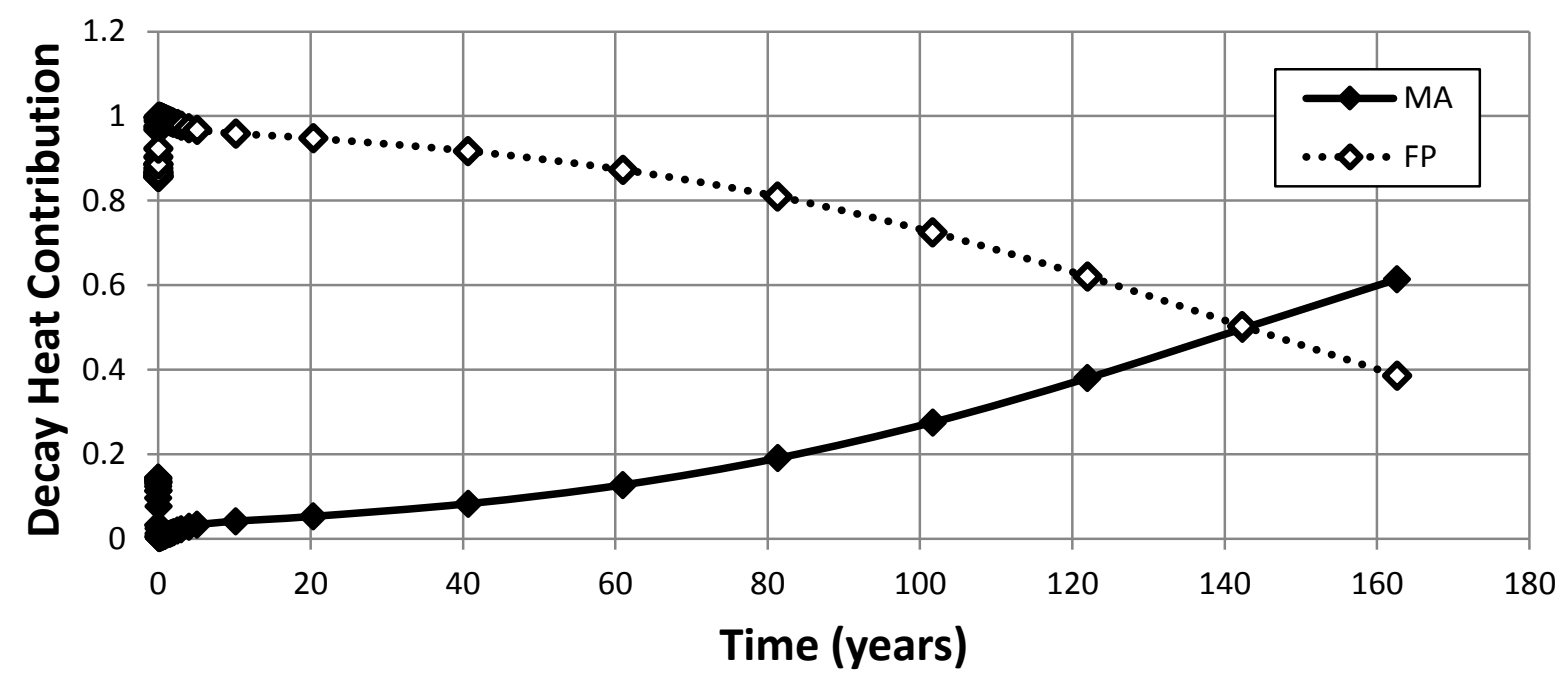

Figure 31. Fractional decay heat contribution from MAs and FPs (Design B).

\section{$\underline{\text { Dose Rates }}$}

The calculated neutron and photon dose rates are of the same order of magnitude as those seen in Design A. Figures 32 and 33 show the dose rates radially out from the core centerline and axially above the fuel, respectively. Encasing the core in a biological shield appears to be necessary in order to properly mitigate the radiation dose to the core surroundings.

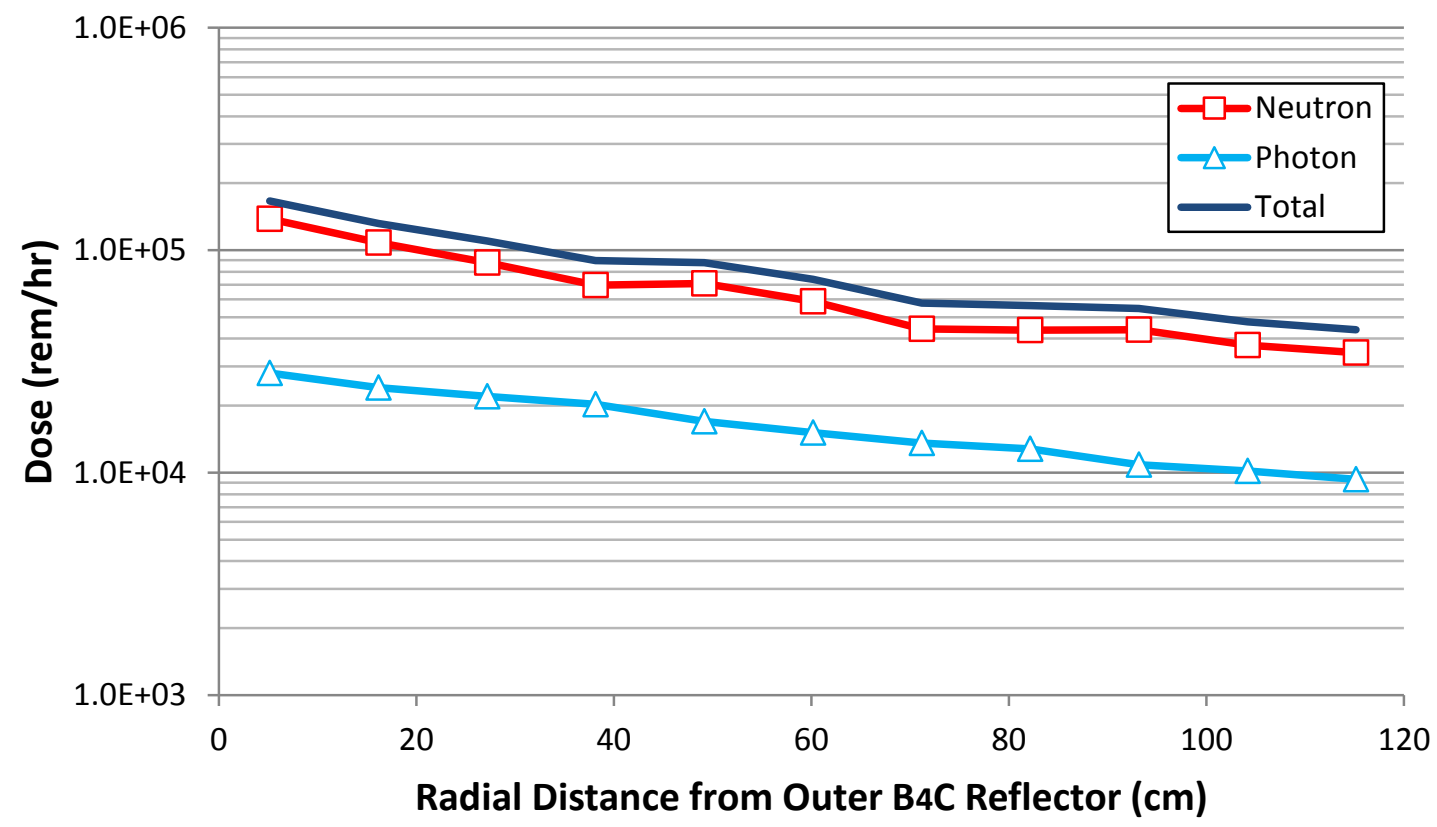

Figure 32. Neutron and photon dose rates at the core mid-plane outside of the reactor shield (Design B). 


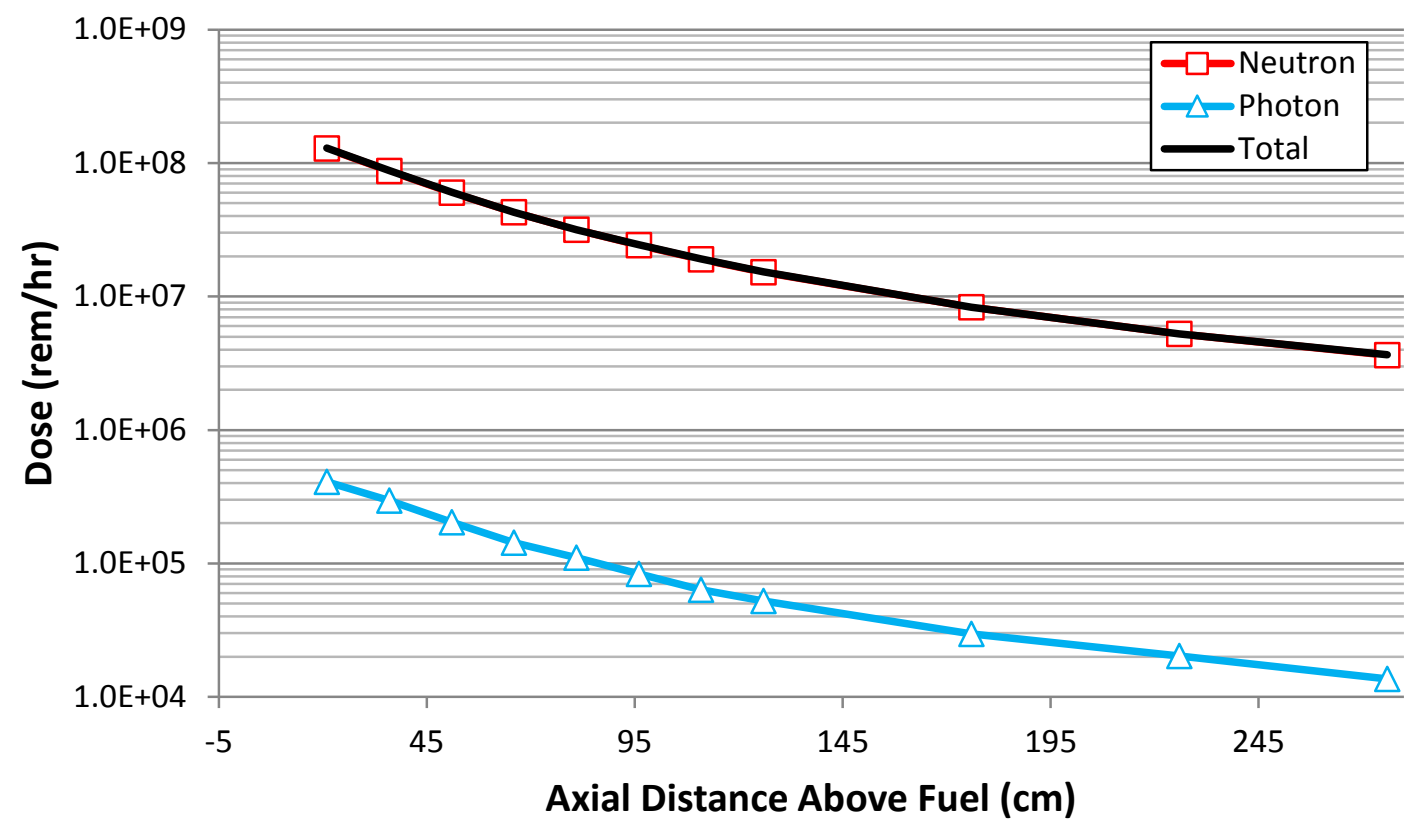

Figure 33. Neutron and photon dose rates above the core (Design B).

\section{$\underline{\mathrm{UO}}_{2}$ Oxide Fuel versus U-10Zr Metallic Fuel}

The last sensitivity study performed on the Design B core involved replacing the $\mathrm{UO}_{2}$ oxide fuel with U$10 \mathrm{Zr}$ metallic fuel. Although $\mathrm{UO}_{2}$ is the most qualified reactor fuel with a high technology readiness level, U-10Zr fuel has been successfully used in fast spectrum reactors such as the Experimental Breeder Reactors at INL. And due to the higher density theoretical density of $U-10 Z r$ at $16.0 \mathrm{~g} / \mathrm{cm}^{3}$ versus 10.96 $\mathrm{g} / \mathrm{cm}^{3}$ for UO2, there will be a much higher U-235 loading for similar enrichments. Two parametric studies were considered here.

A first parametric study how much the fuel pellet radius could be reduced to match the beginning-of-life excess core reactivity to the nominal $\mathrm{UO}_{2}$ case. This was examined by replacing the $\mathrm{UO}_{2}$ oxide fuel with the equivalently enriched, but much denser, U-10Zr metallic fuel. The pitch and all other dimensions were held constant. Table 20 shows that the fuel pellet radius can be reduced from the nominal $0.746 \mathrm{~cm}$ radius to $0.666 \mathrm{~cm}$, or a radius decrease of nearly $0.8 \mathrm{~mm}$, while maintaining the same BOL core excess reactivity. The net core uranium loading will also decrease by a significant $1397 \mathrm{~kg}(1.397 \mathrm{MT})$. In this parametric study, the U-10Zr density of assumed to be $14.5 \mathrm{~g} / \mathrm{cm}^{3}$ with as assumed porosity of approximately $9.1 \%$. This amount of porosity may be too high for the very low burnup Special Purpose Reactor. A much smaller porosity leading to a $\mathrm{U}-10 \mathrm{Zr}$ density closer to $16.0 \mathrm{~g} / \mathrm{cm}^{3}$ could further reduce the fuel pin radius.

Table 20. Core k-effective as the fuel pellet radius decreases (Design B loaded with U-10Zr).

\begin{tabular}{|l|l|l|l|l|l|}
\hline $\begin{array}{l}\text { Fuel Pellet } \\
\text { Radius }(\mathrm{cm})\end{array}$ & $\begin{array}{l}\text { Fuel Pin } \\
\text { Volume }\left(\mathrm{cm}^{3}\right)\end{array}$ & $\begin{array}{l}\text { U-10Zr } \\
(\mathrm{kg})\end{array}$ & $\begin{array}{l}\mathrm{U} \\
(\mathrm{kg})\end{array}$ & $\begin{array}{l}\mathrm{U}-235 \\
(\mathrm{~kg})\end{array}$ & k-effective \\
\hline 0.746 & 262 & 7645 & 6881 & 1359 & 1.10880 \\
\hline 0.736 & 255 & 7442 & 6698 & 1323 & 1.09871 \\
\hline 0.726 & 248 & 7241 & 6517 & 1287 & 1.08852 \\
\hline 0.716 & 242 & 7043 & 6339 & 1252 & 1.07819 \\
\hline
\end{tabular}




\begin{tabular}{|l|l|l|l|l|l|}
\hline 0.706 & 235 & 6848 & 6163 & 1217 & 1.06771 \\
\hline 0.696 & 228 & 6655 & 5989 & 1183 & 1.05699 \\
\hline 0.686 & 222 & 6465 & 5819 & 1149 & 1.04622 \\
\hline 0.676 & 215 & 6278 & 5650 & 1116 & 1.03525 \\
\hline 0.666 & 209 & 6094 & 5484 & 1083 & 1.02408 \\
\hline 0.656 & 203 & 5912 & 5321 & 1051 & 1.01286 \\
\hline
\end{tabular}

Keeping the nominal radii of $0.746 \mathrm{~cm}$, the $\mathrm{U} 10 \mathrm{Zr}$ fuel enrichment could be reduced to roughly 16.63 wt $\%$ to maintain an equivalent BOL core reactivity, as seen in Figure 34.

The second parametric study simply reduced the U-235 enrichment to match beginning-of-life excess reactivity with the nominal $\mathrm{UO}_{2}$ case. The nominal $\mathrm{UO}_{2}$ fuel pellet radius of $0.746 \mathrm{~cm}$ was held constant and the U-235 enrichment varied. Figure 34 shows the core k-effective as the U-235 enrichment decreases assuming a $16.0 \mathrm{~g} / \mathrm{cm}^{3}$ density for the $\mathrm{U}-10 \mathrm{Zr}$. An equivalent beginning-of-life excess reactivity is obtained with if the U-10Zr enrichment is reduced to $<15.0 \mathrm{wt} \% \mathrm{U}-235$. The U-10Zr therefore affords approximately a $25 \%$ decrease in enrichment, a significant decrease from $19.75 \mathrm{wt} \%$ U-235.

If the $\mathrm{U}-10 \mathrm{Zr}$ density is assumed to be $14.4 \mathrm{~g} / \mathrm{cm} 3$ ( $10 \%$ porosity), the $\mathrm{U}-235$ enrichment can be reduced to approximately $16 \mathrm{wt} \% \mathrm{U}-235$, again a significant $20 \%$ decrease in enrichment. The actual porosity needed for the Special Purpose Reactor will probably be between 0 and 10\% porosity, and perhaps significantly closer to $0 \%$, because of the low burnup of the fuel over 5 years.

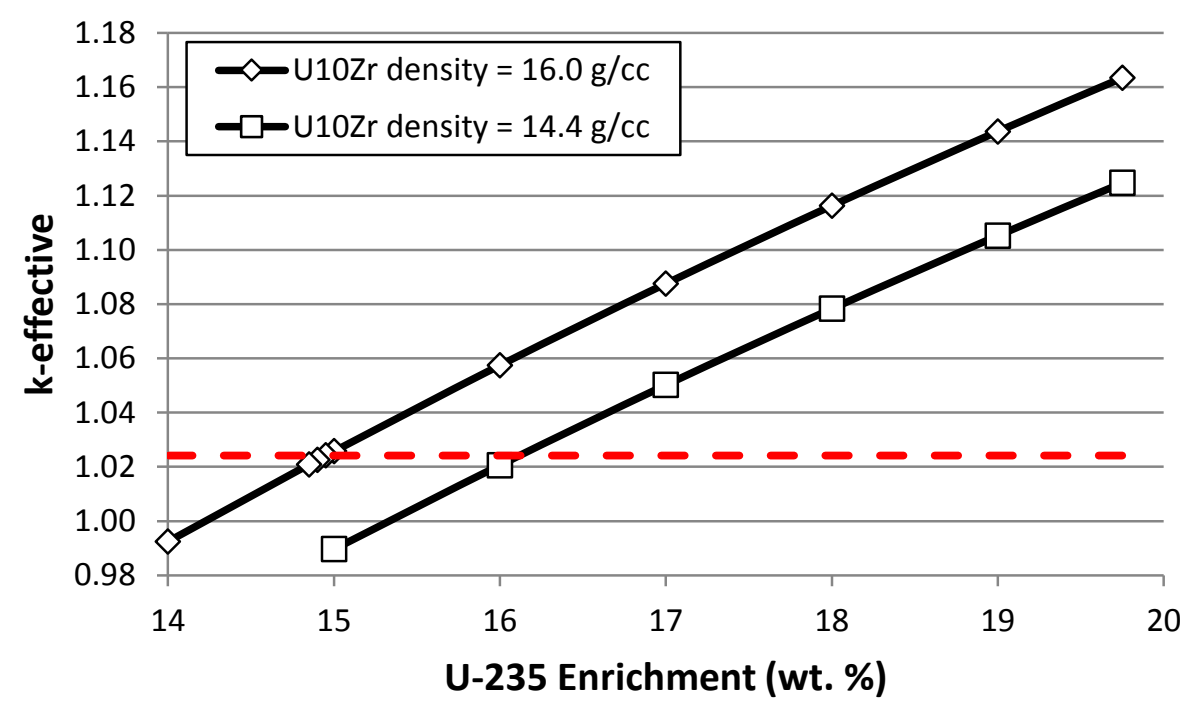

Figure 34. Design B core k-effective versus U-235 enrichment with U-10Zr metallic fuel. 


\section{Appendix C}

\section{Thermal Analysis -- Design A}

This appendix presents details of a preliminary thermal analysis for Design A. Included in this appendix are descriptions of the computer codes, computer models, and assumptions used to perform the parametric studies in order to evaluate Design A reactor thermal sensitivities and characteristics to help evaluate and support the overall Design A reactor system. 


\section{Design A}

\section{TABLE OF CONTENTS}

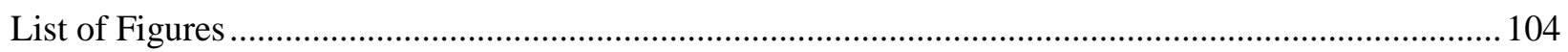

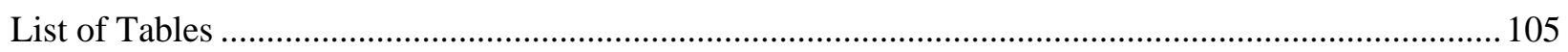

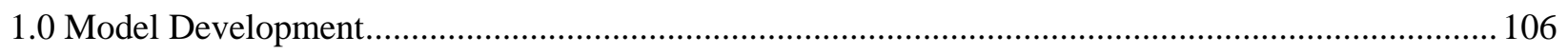

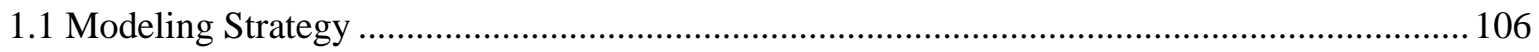

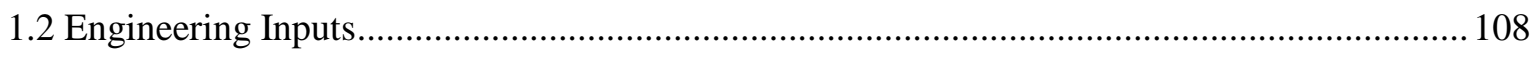

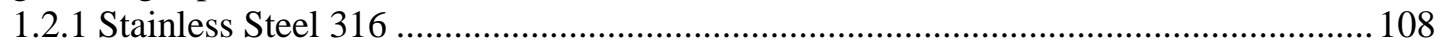

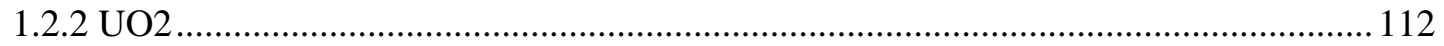

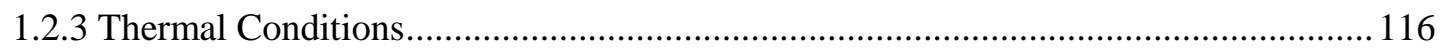

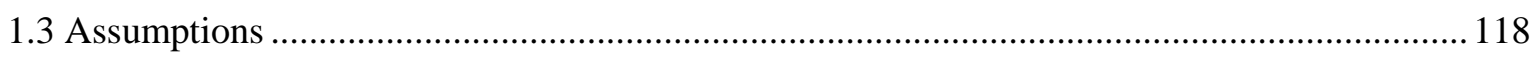

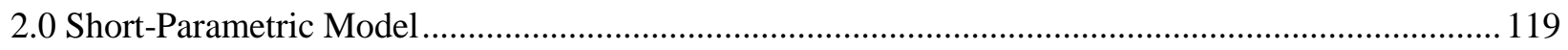

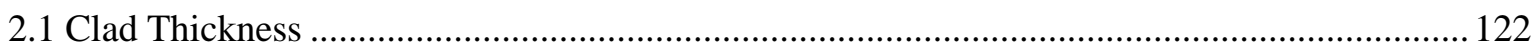

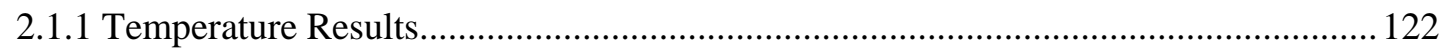

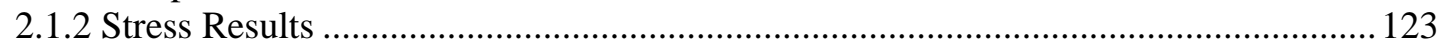

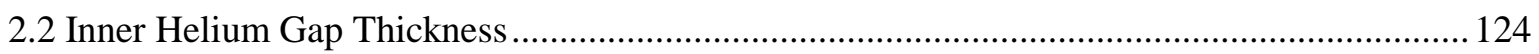

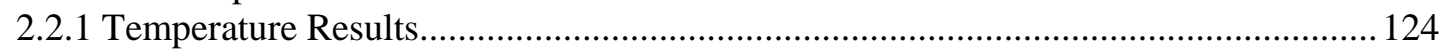

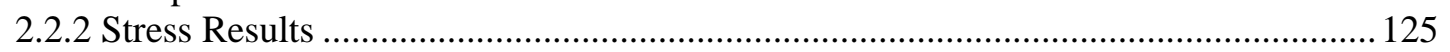

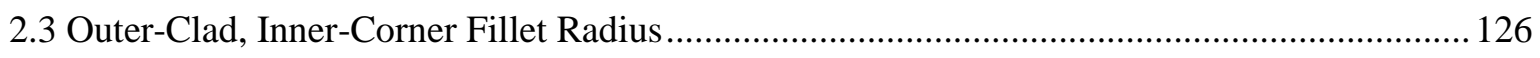

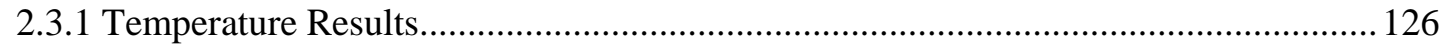

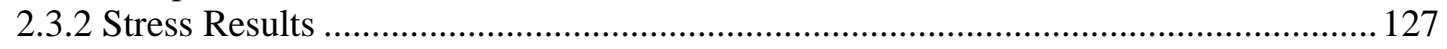

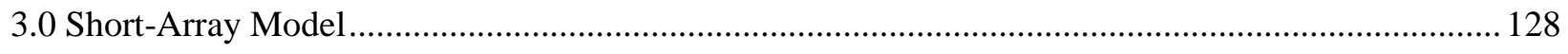

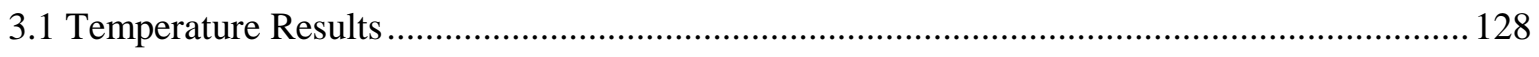

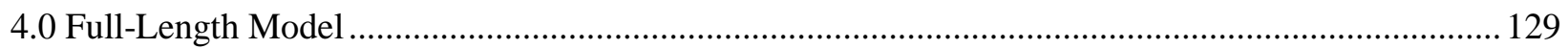

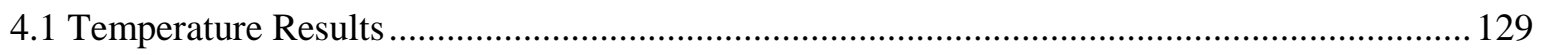

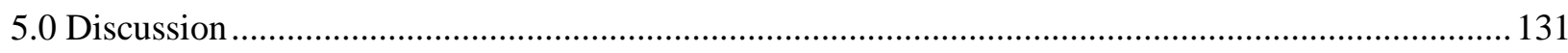

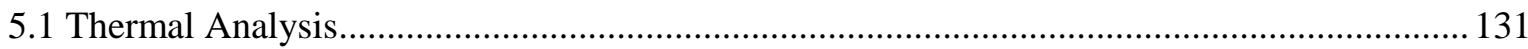

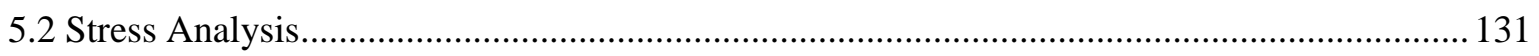

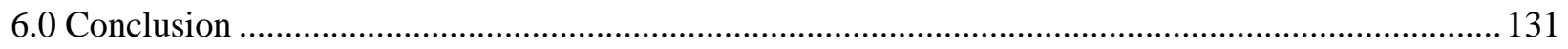

\section{List of Figures}

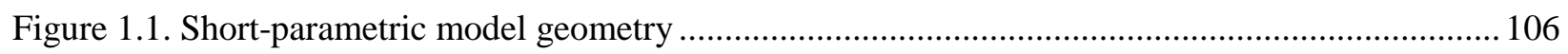

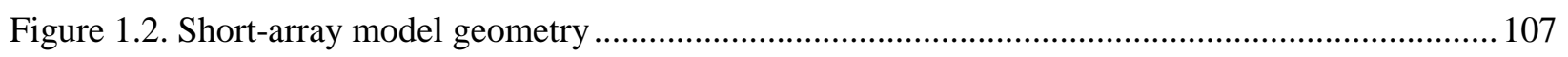

Figure 1.3. Full-length model geometry - outer element view (top); cross-section (bottom) ................... 107

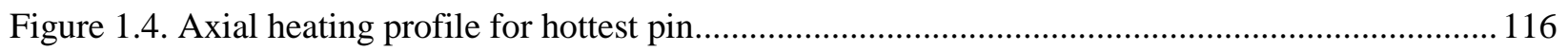

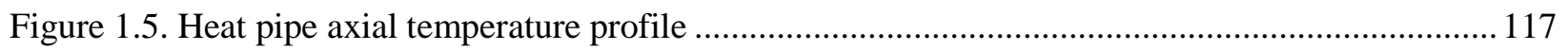




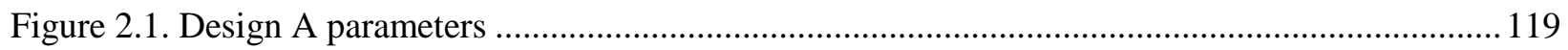

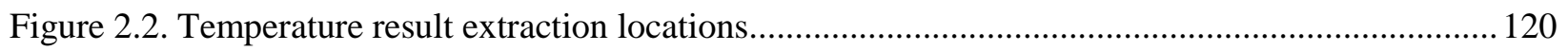

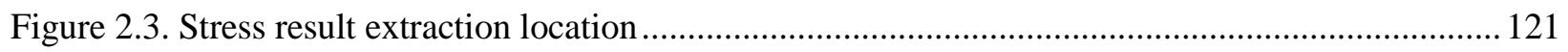

Figure 2.4. Change in clad temperatures as clad thickness is varied .................................................. 122

Figure 2.5. Change in fuel temperatures as clad thickness is varied.................................................... 122

Figure 2.6. Change in clad stress as clad thickness is varied ............................................................ 123

Figure 2.7. Change in peak fuel stress as clad thickness is varied ..................................................... 123

Figure 2.8. Change in clad temperatures as inner helium gap thickness is varied................................. 124

Figure 2.9. Change in fuel temperatures as inner helium gap thickness is varied ................................. 124

Figure 2.10. Change in clad stress as inner helium gap thickness is varied......................................... 125

Figure 2.11. Change in peak fuel stress as inner helium gap thickness is varied ................................. 125

Figure 2.12. Change in clad temperatures as the inner corner fillet radius of the hex clad is varied........ 126

Figure 2.13. Change in fuel temperatures as the inner corner fillet radius of the hex clad is varied ........ 126

Figure 2.14. Change in clad stress as the inner corner fillet radius of the hex clad is varied .................. 127

Figure 2.15. Change in peak fuel stress as the inner corner fillet radius of the hex clad is varied ...........127

Figure 3.1. Temperature distribution in the short-array model ........................................................... 128

Figure 4.1. X-Y plane temperature distribution halfway along the fuel in the full-length model ............ 129

Figure 4.2. Y-Z plane temperature distribution in the full-length model.............................................. 130

Figure 4.3. Outer clad temperature distribution in the full-length model .............................................. 130

\section{List of Tables}

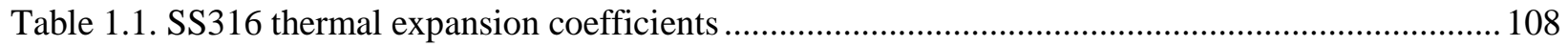

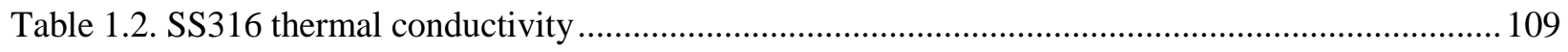

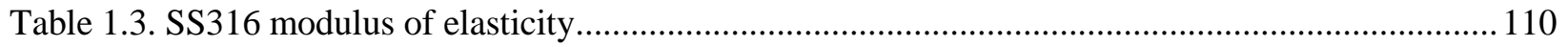

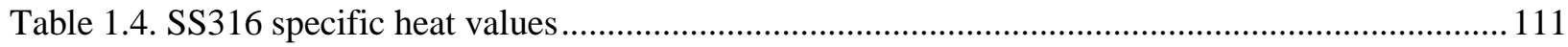

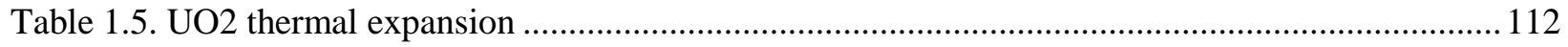

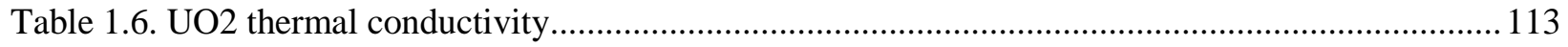

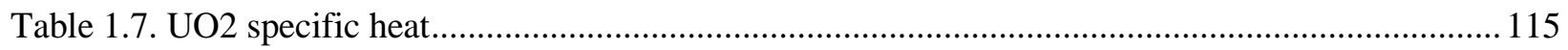

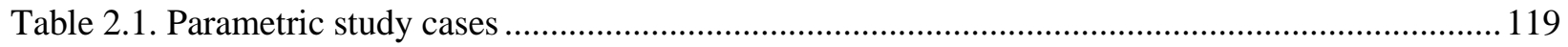




\subsection{Model Development}

\subsection{Modeling Strategy}

Finite element modeling was completed in Abaqus 2016 on the INL Falcon HPC system. Three basic models have been developed and analyzed:

- Short-parametric model (Figure 1.1): represents a 4-cm long segment of a single element. It is used for parametric analysis of clad thickness, inner helium gap thickness, and the radius of the fillet on the inner hex clad corners. Heat generation is uniform throughout the fuel region. Thermal expansion is disabled in the axial $(\mathrm{Z})$ direction. The small size of this model allows each parametric simulation to run relatively quickly.

- Short-array model (Figure 1.2): represents an array of seven, 4-cm long elements in a cluster. This model has all of the same characteristics as mentioned for the short-parametric model. The increased contact calculation from the seven elements touching each other resulted in a significant increase in runtime for the simulation. Therefore, only a single analysis was completed with this model.

- Full-length model (Figure 1.3): represents a single, full length element. This model maintained the same grid density used in the short models. The volumetric heat generation rate varies with the axial location, producing a realistic temperature distribution.

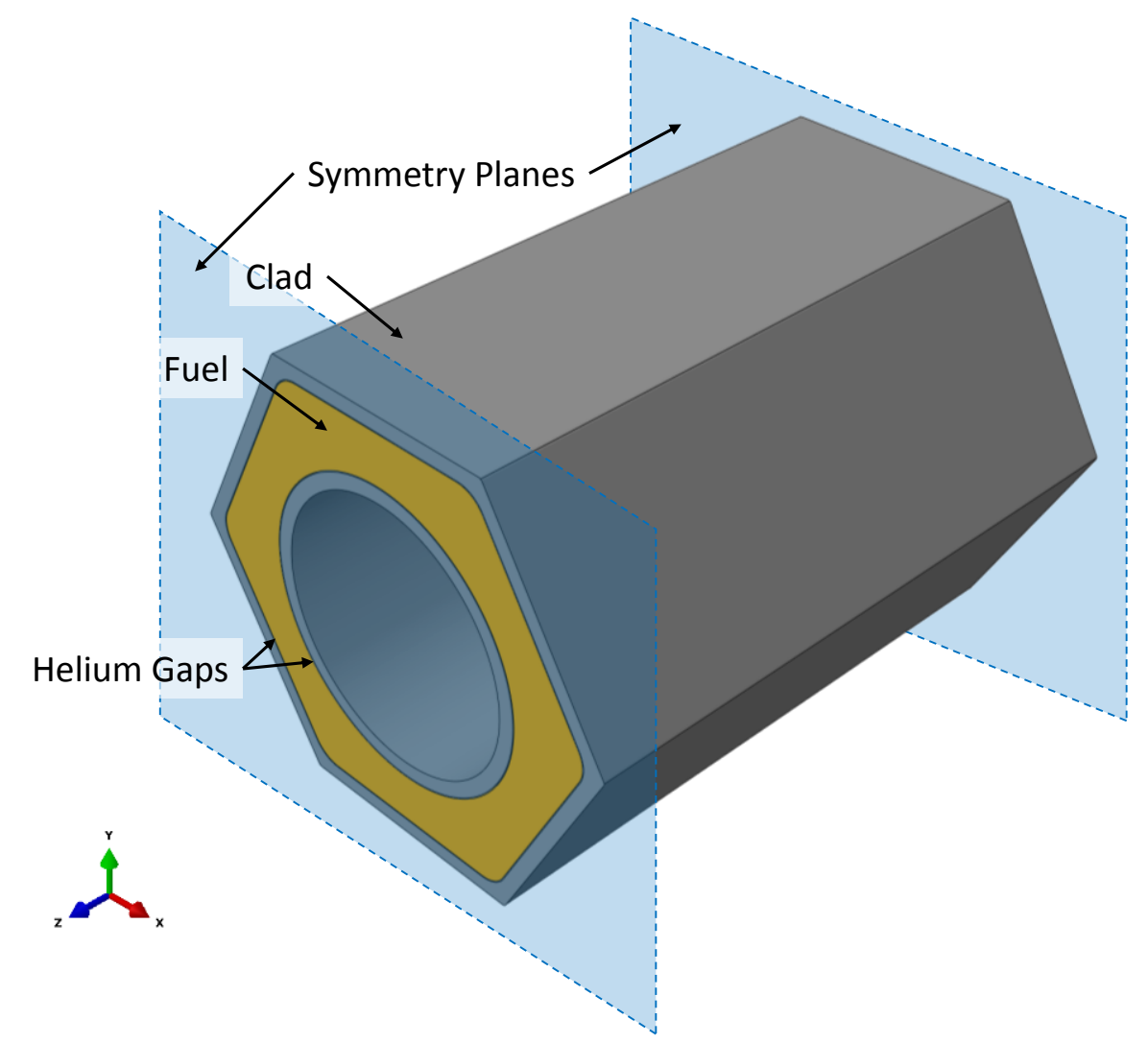

Figure 1.1. Short-parametric model geometry 


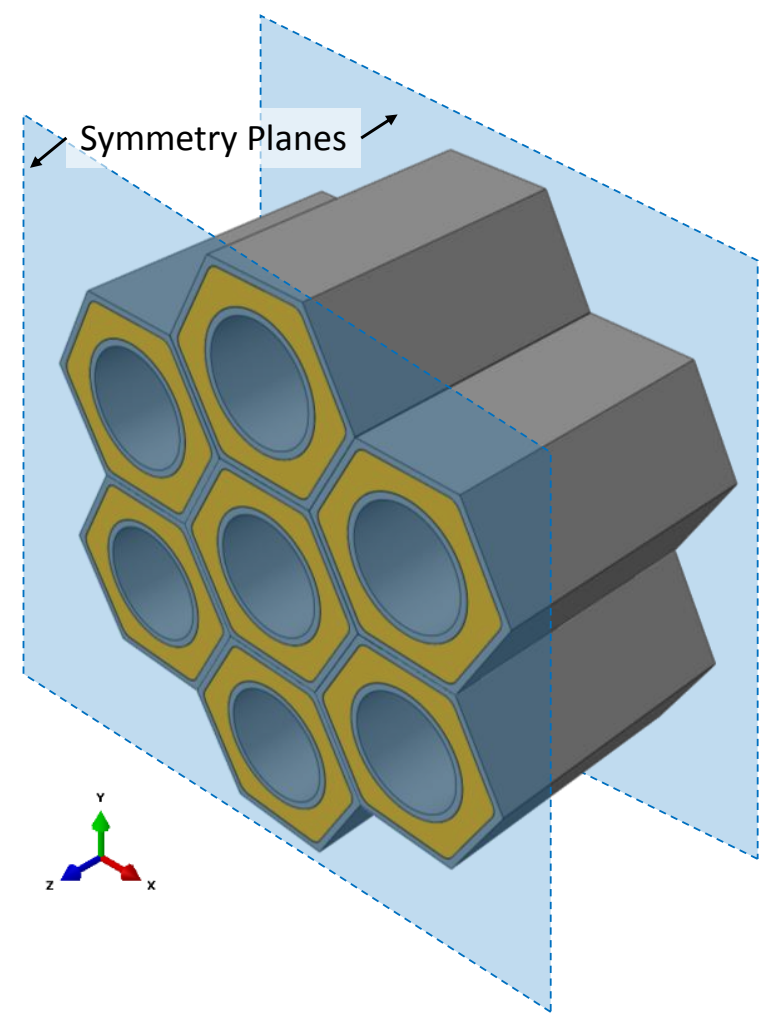

Figure 1.2. Short-array model geometry

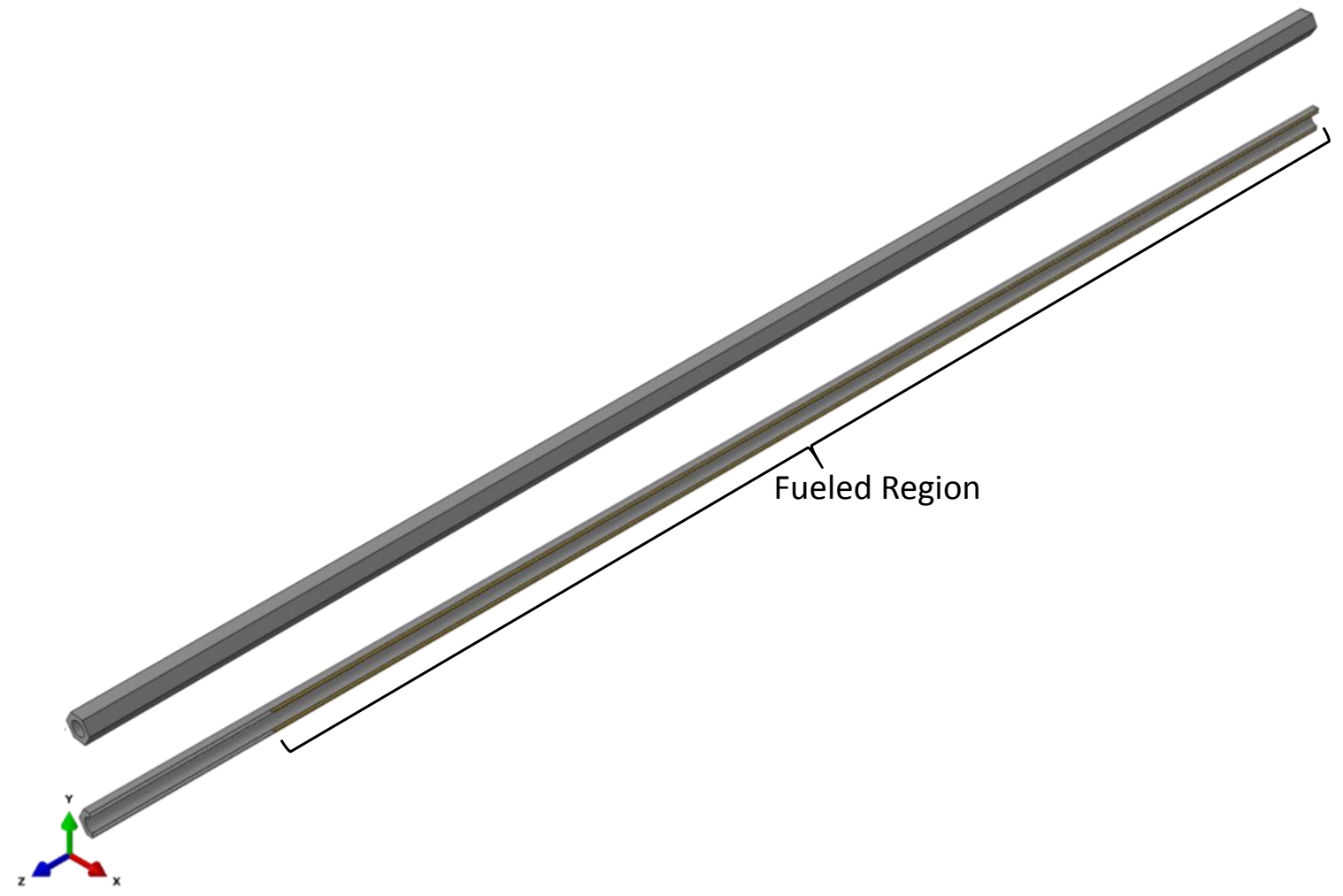

Figure 1.3. Full-length model geometry - outer element view (top); cross-section (bottom) 


\subsection{Engineering Inputs}

This section details the material properties and thermal boundary conditions.

\subsubsection{Stainless Steel 316}

\subsubsection{Thermal Expansion}

From: 2017 ASME BPVC Section II Part D (Metric), Table TE-1, "Coefficients for Austenitic Stainless Steels (Group 3)," pp. 806

Table 1.1. SS316 thermal expansion coefficients

\begin{tabular}{cc}
\hline $\begin{array}{c}\text { Temperature } \\
\mathbf{T}\end{array}$ & $\begin{array}{c}\text { Expansion Coefficient } \\
\mathbf{\alpha A v G} \\
(\mathbf{K}) \mathbf{m} \cdot \mathbf{K})\end{array}$ \\
\hline 293.15 & 0.0000153 \\
323.15 & 0.0000156 \\
348.15 & 0.0000159 \\
373.15 & 0.0000162 \\
398.15 & 0.0000164 \\
423.15 & 0.0000166 \\
448.15 & 0.0000168 \\
473.15 & 0.0000170 \\
498.15 & 0.0000172 \\
523.15 & 0.0000174 \\
548.15 & 0.0000175 \\
573.15 & 0.0000177 \\
598.15 & 0.0000178 \\
623.15 & 0.0000179 \\
648.15 & 0.0000180 \\
673.15 & 0.0000181 \\
698.15 & 0.0000182 \\
723.15 & 0.0000183 \\
748.15 & 0.0000184 \\
773.15 & 0.0000184 \\
798.15 & 0.0000185 \\
823.15 & 0.0000186 \\
848.15 & 0.0000187 \\
873.15 & 0.0000188 \\
898.15 & 0.0000189 \\
923.15 & 0.0000190 \\
948.15 & 0.0000191 \\
973.15 & 0.0000192 \\
998.15 & 0.0000193 \\
1023.15 & 0.0000194 \\
1048.15 & 0.0000194 \\
1073.15 & 0.0000194 \\
1098.15 & 0.0000194 \\
\hline & \\
\hline
\end{tabular}

\subsubsection{Thermal Conductivity}

From: 2017 ASME BPVC Section II Part D (Metric), Table TCD, "High Alloy Steels: Material Group K," pp. 823 
Table 1.2. SS316 thermal conductivity

\begin{tabular}{|c|c|}
\hline $\begin{array}{c}\text { Temperature } \\
\mathbf{T} \\
(\mathbf{K}) \\
\end{array}$ & $\begin{array}{c}\text { Thermal Conductivity } \\
\mathbf{k} \\
(\mathrm{W} / \mathbf{m} \cdot \mathbf{K}) \\
\end{array}$ \\
\hline 293.15 & 14.1 \\
\hline 323.15 & 14.6 \\
\hline 348.15 & 15.0 \\
\hline 373.15 & 15.4 \\
\hline 398.15 & 15.7 \\
\hline 423.15 & 16.1 \\
\hline 448.15 & 16.5 \\
\hline 473.15 & 16.8 \\
\hline 498.15 & 17.2 \\
\hline 523.15 & 17.6 \\
\hline 548.15 & 17.9 \\
\hline 573.15 & 18.3 \\
\hline 598.15 & 18.7 \\
\hline 623.15 & 19.0 \\
\hline 648.15 & 19.4 \\
\hline 673.15 & 19.7 \\
\hline 698.15 & 20.1 \\
\hline 723.15 & 20.5 \\
\hline 748.15 & 20.8 \\
\hline 773.15 & 21.2 \\
\hline 798.15 & 21.5 \\
\hline 823.15 & 21.9 \\
\hline 848.15 & 22.2 \\
\hline 873.15 & 22.6 \\
\hline 898.15 & 22.9 \\
\hline 923.15 & 23.2 \\
\hline 948.15 & 23.6 \\
\hline 973.15 & 23.9 \\
\hline 998.15 & 24.2 \\
\hline
\end{tabular}




\subsubsection{Elastic Modulus}

From: 2017 ASME BPVC Section II Part D (Metric), Table TM-1, "Moduli of Elasticity E for Ferrous Materials for Given Temperatures: Material Group G," pp. 835

Table 1.3. SS316 modulus of elasticity

\begin{tabular}{cc}
\hline $\begin{array}{c}\text { Temperature } \\
\mathbf{T}\end{array}$ & $\begin{array}{c}\text { Modulus of Elasticity } \\
\mathbf{K})\end{array}$ \\
$\mathbf{E}$ \\
\hline 73.15 & $2.09 \mathrm{E}+11$ \\
148.15 & $2.04 \mathrm{E}+11$ \\
198.15 & $2.01 \mathrm{E}+11$ \\
298.15 & $1.95 \mathrm{E}+11$ \\
373.15 & $1.89 \mathrm{E}+11$ \\
423.15 & $1.86 \mathrm{E}+11$ \\
473.15 & $1.83 \mathrm{E}+11$ \\
523.15 & $1.79 \mathrm{E}+11$ \\
573.15 & $1.76 \mathrm{E}+11$ \\
623.15 & $1.72 \mathrm{E}+11$ \\
673.15 & $1.69 \mathrm{E}+11$ \\
723.15 & $1.65 \mathrm{E}+11$ \\
773.15 & $1.6 \mathrm{E}+11$ \\
823.15 & $1.56 \mathrm{E}+11$ \\
873.15 & $1.51 \mathrm{E}+11$ \\
923.15 & $1.46 \mathrm{E}+11$ \\
973.15 & $1.4 \mathrm{E}+11$ \\
\hline
\end{tabular}

\subsubsection{Poisson's Ratio \& Density}

From: 2017 ASME BPVC Section II Part D (Metric), Table PRD, "Poisson's Ratio and Density of Materials," pp. 841

Poisson's ratio: $v=0.31$

Density: $\quad \rho=8030 \mathrm{~kg} / \mathrm{m}^{3}$. 


\subsubsection{Specific Heat}

Using the values for thermal conductivity in Table 1.2 , a constant density of $8030 \mathrm{~kg} / \mathrm{m}^{3}$, and thermal diffusivity values from the 2017 ASME BPVC Section II Part D (Metric), Table TCD, "High Alloy Steels, Material Group K,” pp. 823, specific heat was calculated using equation 1.

$$
c_{p}=\frac{k}{\rho \cdot T D}
$$

Table 1.4. SS316 specific heat values

\begin{tabular}{|c|c|c|}
\hline $\begin{array}{c}\text { Temperature } \\
\mathbf{T} \\
(\mathbf{K}) \\
\end{array}$ & $\begin{array}{c}\text { Thermal Diffusivity } \\
\text { TD } \\
\left(\mathrm{m}^{2} / \mathrm{s}\right)\end{array}$ & $\begin{array}{c}\text { Specific Heat } \\
\mathbf{c}_{\mathbf{p}} \\
(\mathrm{J} / \mathrm{kg} \cdot \mathrm{K})\end{array}$ \\
\hline 293.15 & 0.00000357 & 491.8530301 \\
\hline 323.15 & 0.00000364 & 499.5004995 \\
\hline 348.15 & 0.00000369 & 506.2317124 \\
\hline 373.15 & 0.00000375 & 511.4155251 \\
\hline 398.15 & 0.0000038 & 514.5179262 \\
\hline 423.15 & 0.00000386 & 519.4252124 \\
\hline 448.15 & 0.00000392 & 524.1822756 \\
\hline 473.15 & 0.00000398 & 525.6669399 \\
\hline 498.15 & 0.00000405 & 528.8808942 \\
\hline 523.15 & 0.00000411 & 533.2800053 \\
\hline 548.15 & 0.00000416 & 535.8511352 \\
\hline 573.15 & 0.00000422 & 540.0364746 \\
\hline 598.15 & 0.00000428 & 544.1044681 \\
\hline 623.15 & 0.00000433 & 546.449659 \\
\hline 648.15 & 0.00000439 & 550.3280693 \\
\hline 673.15 & 0.00000444 & 552.5450731 \\
\hline 698.15 & 0.0000045 & 556.2474056 \\
\hline 723.15 & 0.00000455 & 561.0827529 \\
\hline 748.15 & 0.00000461 & 561.8842572 \\
\hline 773.15 & 0.00000466 & 566.5449842 \\
\hline 798.15 & 0.00000472 & 567.2583743 \\
\hline 823.15 & 0.00000478 & 570.559148 \\
\hline 848.15 & 0.00000484 & 571.2050884 \\
\hline 873.15 & 0.0000049 & 574.3766996 \\
\hline 898.15 & 0.00000495 & 576.1223694 \\
\hline 923.15 & 0.00000501 & 576.6797662 \\
\hline 948.15 & 0.00000507 & 579.6802425 \\
\hline 973.15 & 0.00000512 & 581.3161582 \\
\hline 998.15 & 0.00000516 & 584.0501221 \\
\hline 1023.15 & 0.00000519 & 590.272029 \\
\hline
\end{tabular}




\subsubsection{UO2}

\subsubsection{Thermal Expansion}

From: IAEA-TECDOC-1496, "Thermophysical Properties Database of Materials for Light Water Reactors and Heavy Water Reactors," Section 6.1.1.3, Table 1, pp. 56

Table 1.5. UO2 thermal expansion

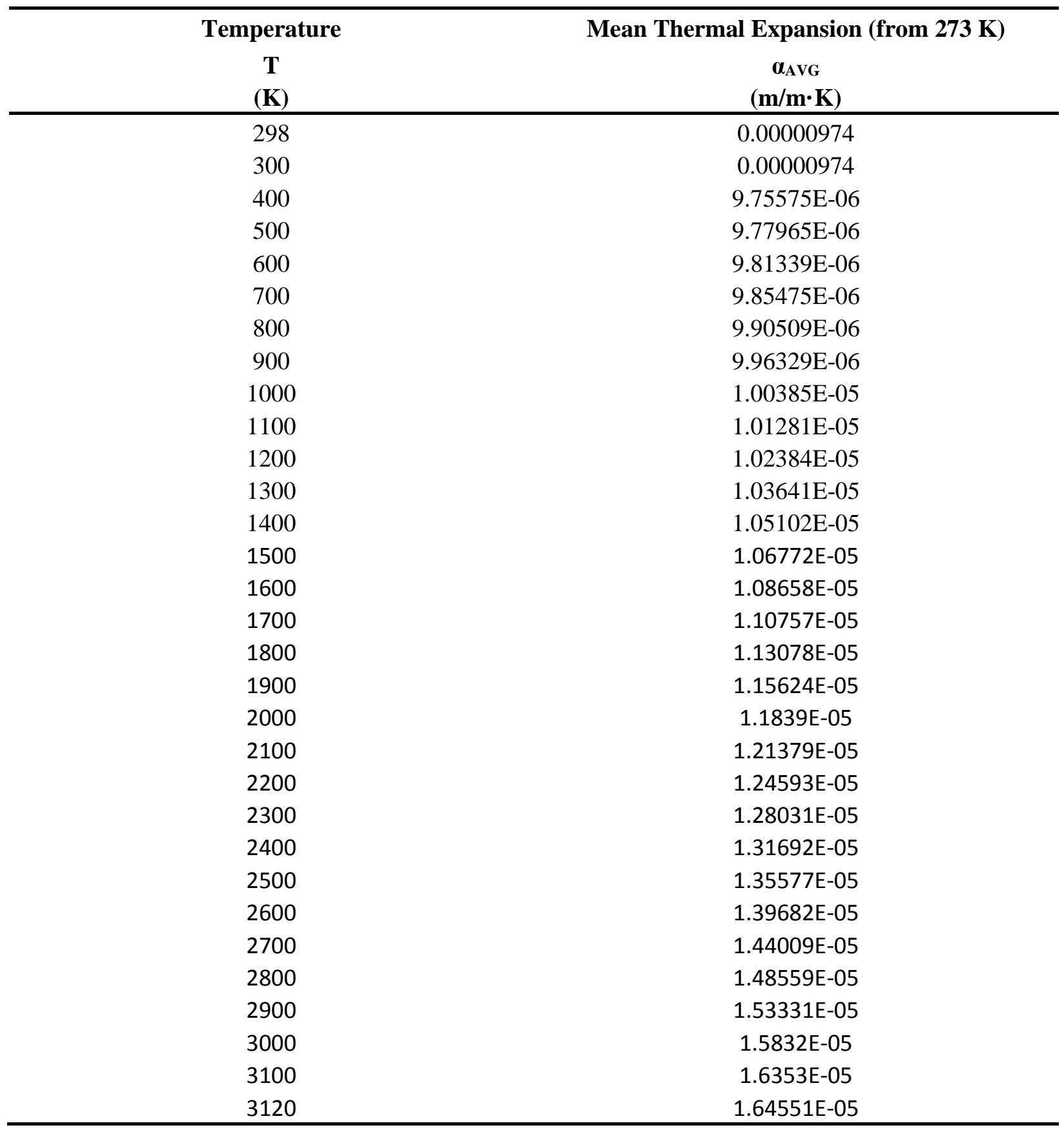




\subsubsection{Thermal Conductivity}

From: IAEA-TECDOC-1496, "Thermophysical Properties Database of Materials for Light Water Reactors and Heavy Water Reactors, Section 6.1.1.7, Table 1, pp. 91

Table 1.6. UO2 thermal conductivity

\begin{tabular}{|c|c|}
\hline $\begin{array}{c}\text { Temperature } \\
\mathbf{T} \\
(\mathbf{K}) \\
\end{array}$ & $\begin{array}{l}\text { Thermal Conductivity (95\% Dense) } \\
\mathbf{k} \\
(\mathrm{W} / \mathbf{m} \cdot \mathbf{K})\end{array}$ \\
\hline 298.15 & 7.61 \\
\hline 300 & 7.59 \\
\hline 400 & 6.58 \\
\hline 500 & 5.78 \\
\hline 600 & 5.14 \\
\hline 700 & 4.61 \\
\hline 800 & 4.17 \\
\hline 900 & 3.79 \\
\hline 1000 & 3.47 \\
\hline 1100 & 3.19 \\
\hline 1200 & 2.95 \\
\hline 1300 & 2.74 \\
\hline 1400 & 2.56 \\
\hline 1500 & 2.41 \\
\hline 1600 & 2.29 \\
\hline 1700 & 2.19 \\
\hline 1800 & 2.12 \\
\hline 1900 & 2.08 \\
\hline 2000 & 2.06 \\
\hline 2100 & 2.07 \\
\hline 2200 & 2.09 \\
\hline 2300 & 2.14 \\
\hline 2400 & 2.20 \\
\hline 2500 & 2.28 \\
\hline 2600 & 2.37 \\
\hline 2700 & 2.48 \\
\hline 2800 & 2.59 \\
\hline 2900 & 2.71 \\
\hline 3000 & 2.84 \\
\hline 3100 & 2.97 \\
\hline 3120 & 2.99 \\
\hline
\end{tabular}




\subsubsection{Elastic Modulus \& Poisson's Ratio}

From: NIST Structural Ceramics Database, SRD Database Number 30,

https://srdata.nist.gov/CeramicDataPortal/Elasticity/UO2

Elastic Modulus: $\quad E=195 \mathrm{GPa}$

Poisson's Ratio: $v=0.31$

\subsubsection{Density}

From: IAEA-TECDOC-1496, "Thermophysical Properties Database of Materials for Light Water Reactors and Heavy Water Reactors," Section 6.1.1.10, Table 1, pp. 115

Density:

$$
\rho=10960 \mathrm{~kg} / \mathrm{m}^{3}
$$




\subsubsection{Specific Heat}

From: IAEA-TECDOC-1496, "Thermophysical Properties Database of Materials for Light Water Reactors and Heavy Water Reactors," Section 6.1.1.1, Table 3, pp. 29

Table 1.7. UO2 specific heat

\begin{tabular}{|c|c|}
\hline $\begin{array}{c}\mathbf{T} \\
(\mathbf{K}) \\
298.15 \\
\end{array}$ & $\begin{array}{c}\mathbf{C}_{\mathbf{p}} \\
(\mathbf{J} / \mathbf{k g} \cdot \mathbf{K}) \\
235 \\
\end{array}$ \\
\hline 300 & 235 \\
\hline 400 & 266 \\
\hline 500 & 282 \\
\hline 600 & 292 \\
\hline 700 & 299 \\
\hline 800 & 304 \\
\hline 900 & 308 \\
\hline 1000 & 312 \\
\hline 1100 & 315 \\
\hline 1200 & 318 \\
\hline 1300 & 320 \\
\hline 1400 & 324 \\
\hline 1500 & 327 \\
\hline 1600 & 332 \\
\hline 1700 & 339 \\
\hline 1800 & 347 \\
\hline 1900 & 358 \\
\hline 2000 & 373 \\
\hline 2100 & 390 \\
\hline 2200 & 411 \\
\hline 2300 & 437 \\
\hline 2400 & 466 \\
\hline 2500 & 500 \\
\hline 2600 & 537 \\
\hline 2700 & 579 \\
\hline 2800 & 625 \\
\hline 2900 & 674 \\
\hline 3000 & 726 \\
\hline 3100 & 781 \\
\hline 3120 & 792 \\
\hline
\end{tabular}




\subsubsection{Thermal Conditions}

A heating profile for the hottest pin was provided at six axial locations from the physics analysis. Figure 1.4 shows the six volumetric heating rates $\left(\mathrm{W} / \mathrm{m}^{3}\right)$ against the left axis, with the curve fit equation.

Additionally, a simple estimate of the total power (W) for each of the six axial fuel pin segments is shown in the bars plotted against the right axis.

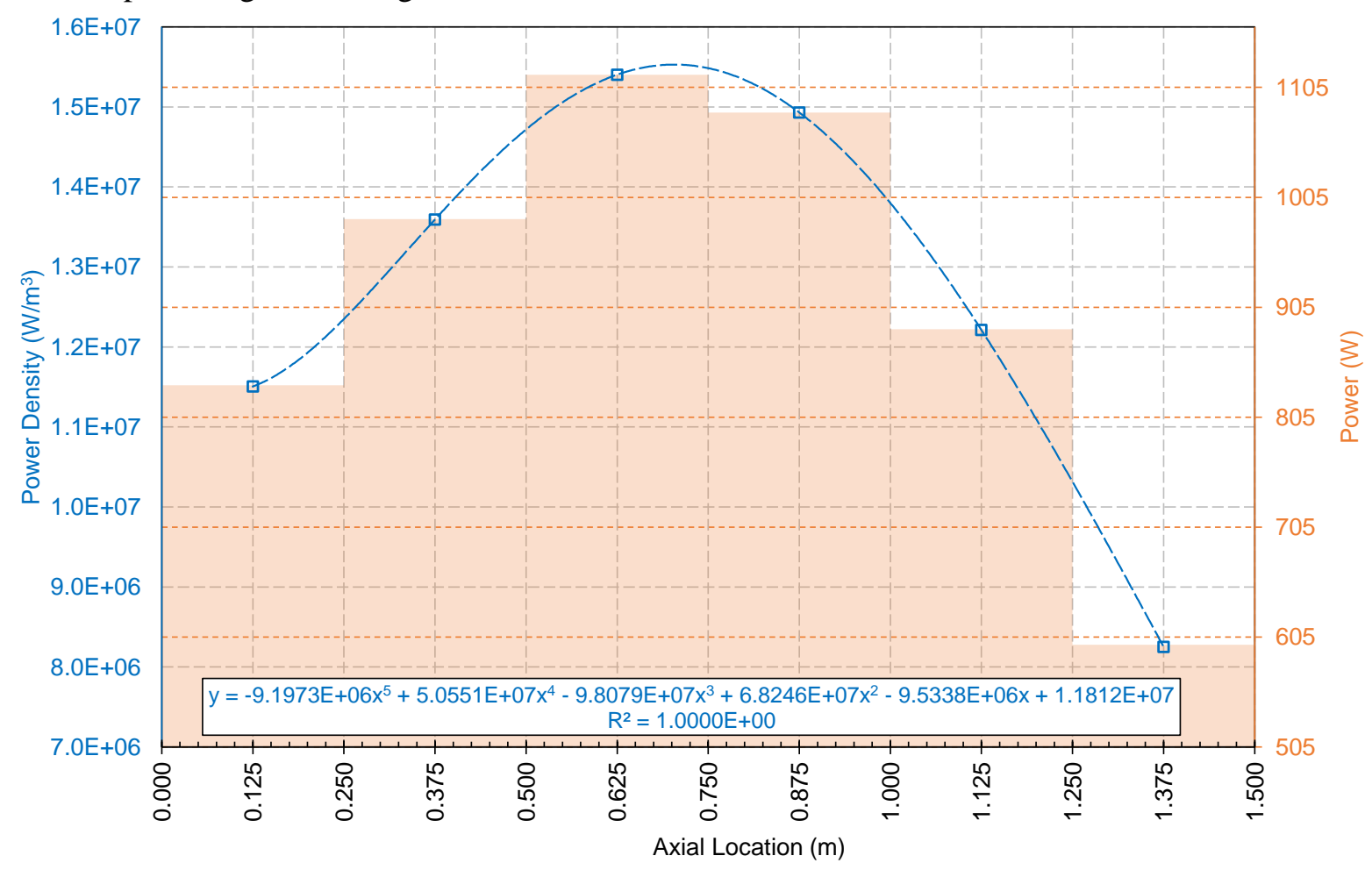

Figure 1.4. Axial heating profile for hottest pin

The peak volumetric heat generation rate of $1.54 \cdot 10^{7} \mathrm{~W} / \mathrm{m}^{3}$ is used uniformly in the $4 \mathrm{~cm}$ long fuel segment of the short models. The equation for the volumetric heat rate as function of axial location is used to create a more representative power distribution in the full length model.

A constant temperature boundary condition of $986 \mathrm{~K}\left(712.5^{\circ} \mathrm{C}\right)$ is specified at the inner surface of the inner clad, where the heat pipe would be located. This is based on the maximum axial heat pipe temperature which occurs at the end of the evaporator section of the heat pipe. Also, no account is taken of the temperature increase across the heat pipe wall and gap between the heat pipe and inner clad. The axial heat pipe temperature profile was calculated with the LANL HTPIPE code and shown in Figure 1.5. Adiabatic conditions are assumed at the outer surface of the hex clad. Therefore, all heat generated in the fuel will flux through the inner clad surface where the temperature boundary condition is specified. 


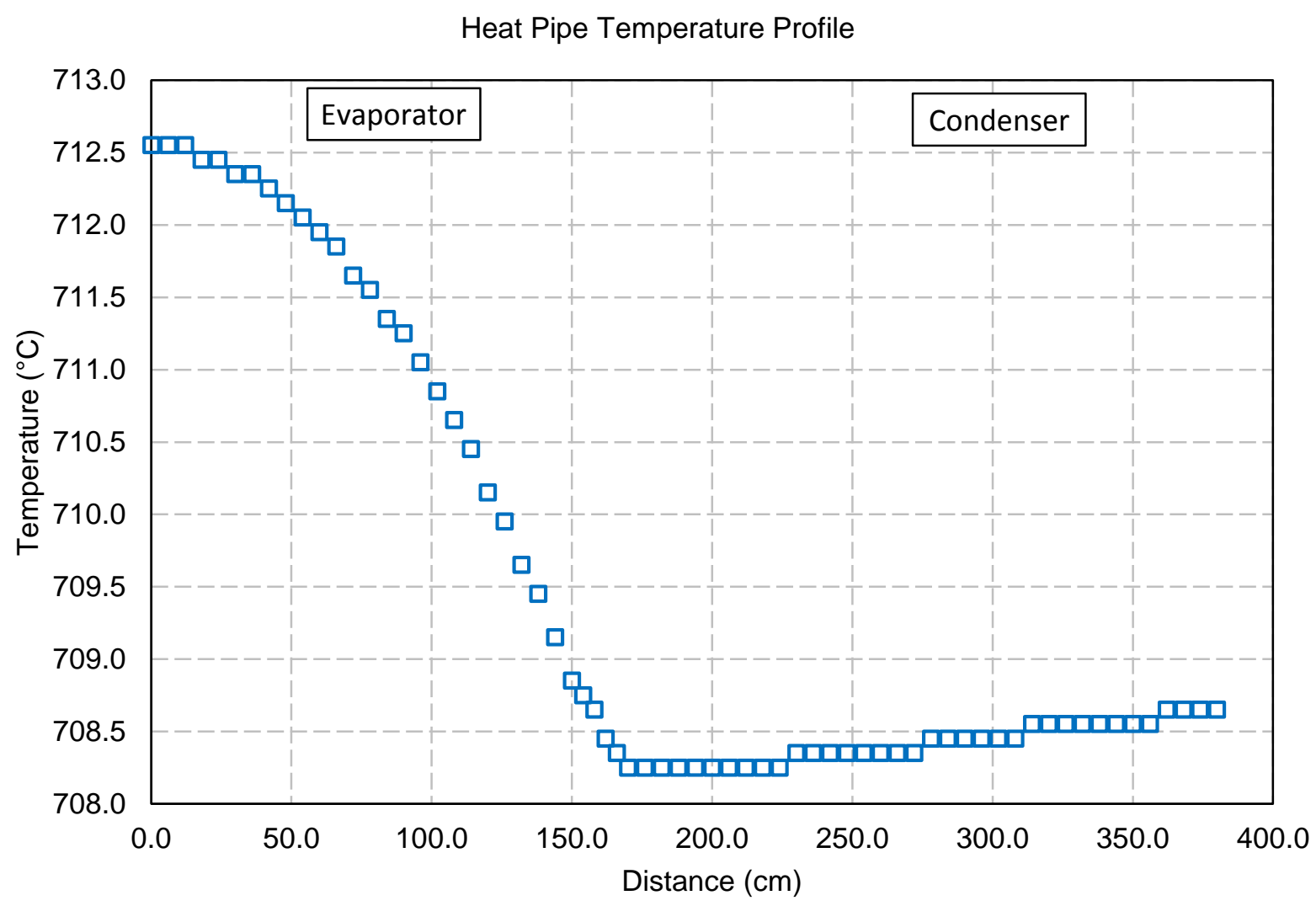

Figure 1.5. Heat pipe axial temperature profile 


\subsection{Assumptions}

For all models presented here, the following assumptions are made:

- All results represent the steady-state condition. No transient analyses have been conducted.

For both the short-parametric and short-array models, the following assumptions are made:

- Heat generation in the fuel is constant throughout the fuel; i.e., the thermal load does not vary with position.

- Symmetry is assumed at the top and bottom surfaces

- Thermal expansion is not calculated axially (z-direction). Thermal expansion is active only in the $\mathrm{X}-\mathrm{Y}$ plane.

- The origin is at the center of the model. Nodes at the $\mathrm{X}=0$ plane, and edges at the $\mathrm{Y}=0$ and $\mathrm{Z}=0$ planes are restricted from moving in their respective normal directions. This has the effect of maintaining symmetric deformations in the model without imposing artificial stresses due to boundary conditions; i.e., the model is free to expand about the origin while maintaining a net zero deflection from the origin.

For the full-length model, the following assumptions are made:

- Heat generation varies as a function of axial location. This is done using the equation shown in Figure 1.4.

- Thermal expansion is active in all directions.

- The origin is at the center of the element in the X-Y plane, and at the end of the element where the fuel contacts the end of the cladding in the $\mathrm{Z}$ plane. Nodes in the $\mathrm{X}=0$ plane, and edges at the $Y=0$ planes are restricted from moving in their respective normal directions. Edges at the fuelclad contact at the $\mathrm{Z}=0$ plane are restricted from moving in the $\mathrm{Z}$ normal direction. This allows for axial elongation of the element from the $\mathrm{Z}=0$ end. 


\subsection{Short-Parametric Model}

The parametric analysis seeks to identify the general trend associated with changing a single parameter. In this analysis, three parameters of interest have been identified - clad thickness, inner helium gap thickness, and the fillet radius on the six inner corners of the outer clad. In order to minimize the change in heat generation as parameters are modified, the fuel inner radius and apothem to the outer surface have been maintained in all models. In the case of changing the corner fillet radius, the fuel volume will change very slightly. This change in fuel volume is considered insignificant over the range of radii studied.

Figure 2.1 shows the parameters used to define the element cross-section. The constant dimensions are in black text, while the variable parameters are in blue text.

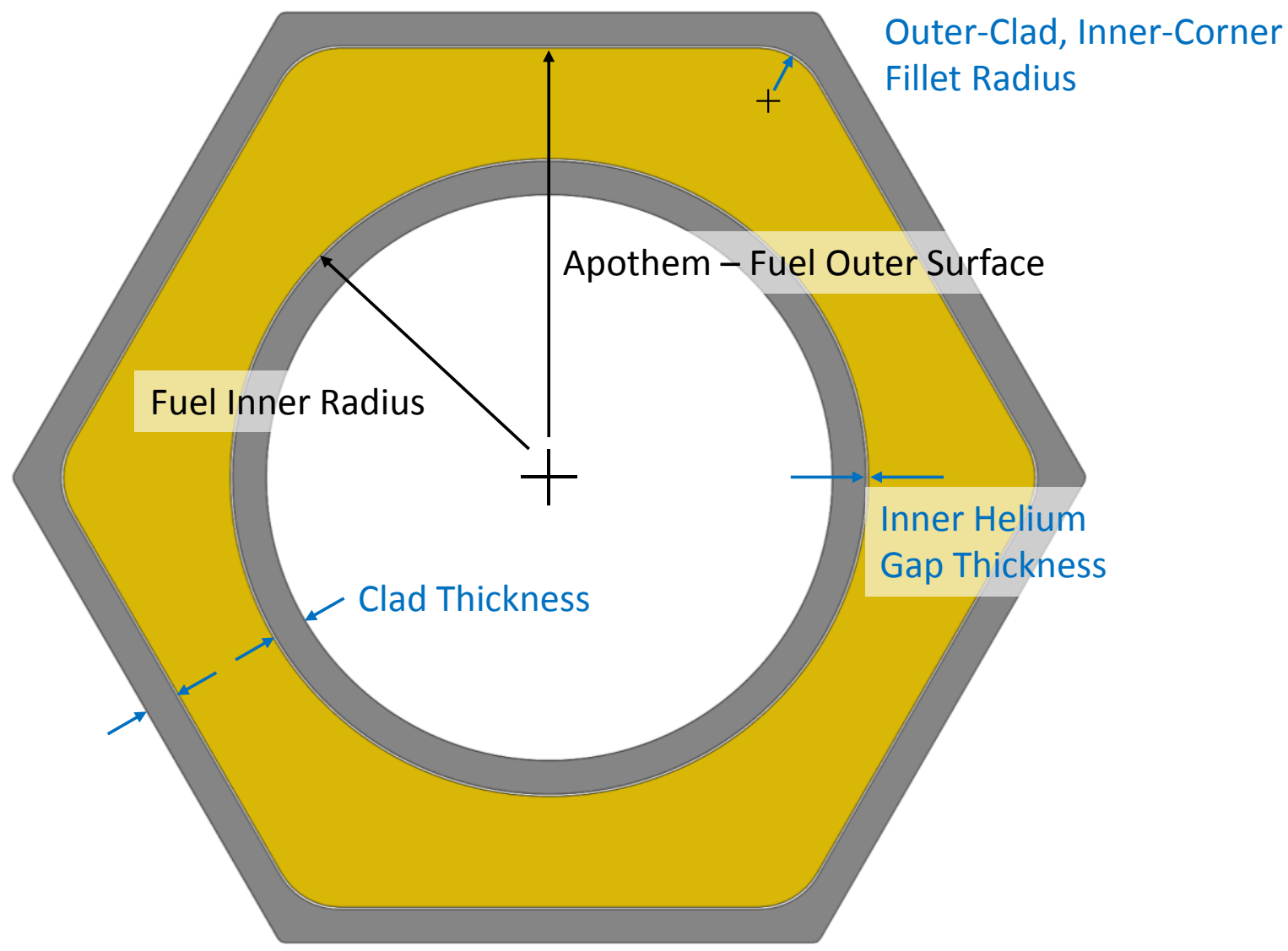

Figure 2.1. Design A parameters

A total of 16 cases have been analyzed for the parametric studies. Table 2.1 shows the variable parameters for each case. The highlighted variables show which dimension was changed for a given case while the other dimensions remained unchanged.

Table 2.1. Parametric study cases

\begin{tabular}{cccc}
\hline Case & Clad Thickness & $\begin{array}{c}\text { Inner Helium Gap } \\
\text { Thickness } \\
(\mathbf{c m})\end{array}$ & $\begin{array}{c}\text { Outer-Clad, Inner-Corner } \\
\text { Fillet Radius } \\
(\mathbf{c m})\end{array}$ \\
\hline 1 & 0.1 & 0.0071 & 0.0254
\end{tabular}




\begin{tabular}{cccc}
2 & 0.1 & 0.0071 & 0.0508 \\
3 & 0.1 & 0.0071 & 0.0762 \\
4 & 0.1 & 0.0071 & 0.1016 \\
5 & 0.1 & 0.0071 & 0.1270 \\
6 & 0.1 & 0.0071 & 0.1524 \\
7 & 0.1 & 0.0071 & 0.1778 \\
8 & 0.1 & 0.0071 & 0.2032 \\
9 & 0.05 & 0.0071 & 0.0254 \\
10 & 0.06 & 0.0071 & 0.0254 \\
11 & 0.07 & 0.0071 & 0.0254 \\
12 & 0.08 & 0.0071 & 0.0254 \\
13 & 0.09 & 0.0271 & 0.0254 \\
14 & 0.1 & 0.0171 & 0.2032 \\
15 & 0.1 & 0.0121 & 0.2032 \\
16 & 0.1 & & 0.2032 \\
\hline
\end{tabular}

Temperature results have been extracted from each case at a number of locations. These locations are noted in Figure 2.2. Note that the temperature of the inner clad at the inside radius is a constant specified boundary condition $\left(712.5^{\circ} \mathrm{C}\right)$.

Stress results have been extracted at the locations noted in Figure 2.3. Additionally, the peak overall stress in the outer hex clad and the fuel have been extracted.

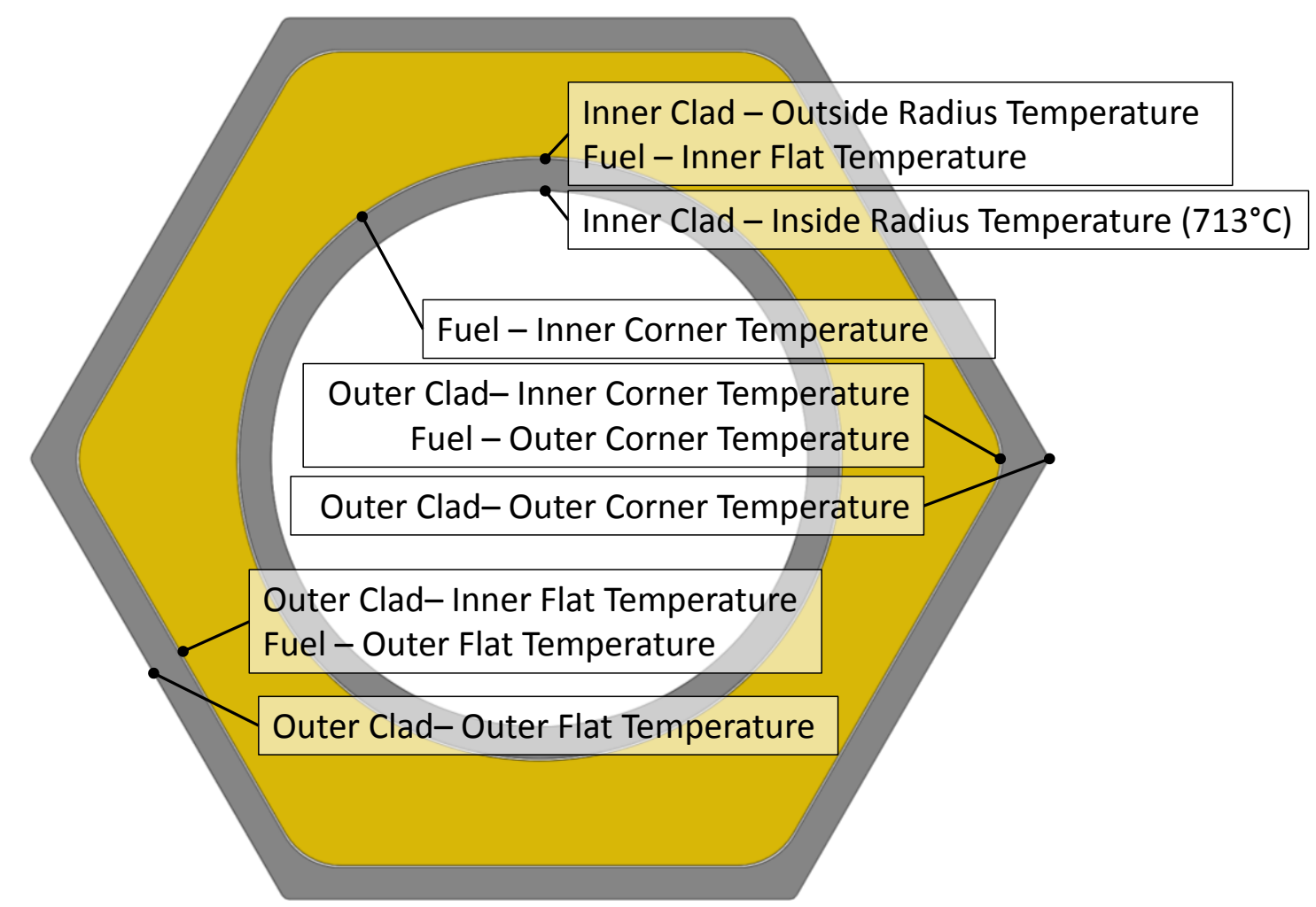

Figure 2.2. Temperature result extraction locations 


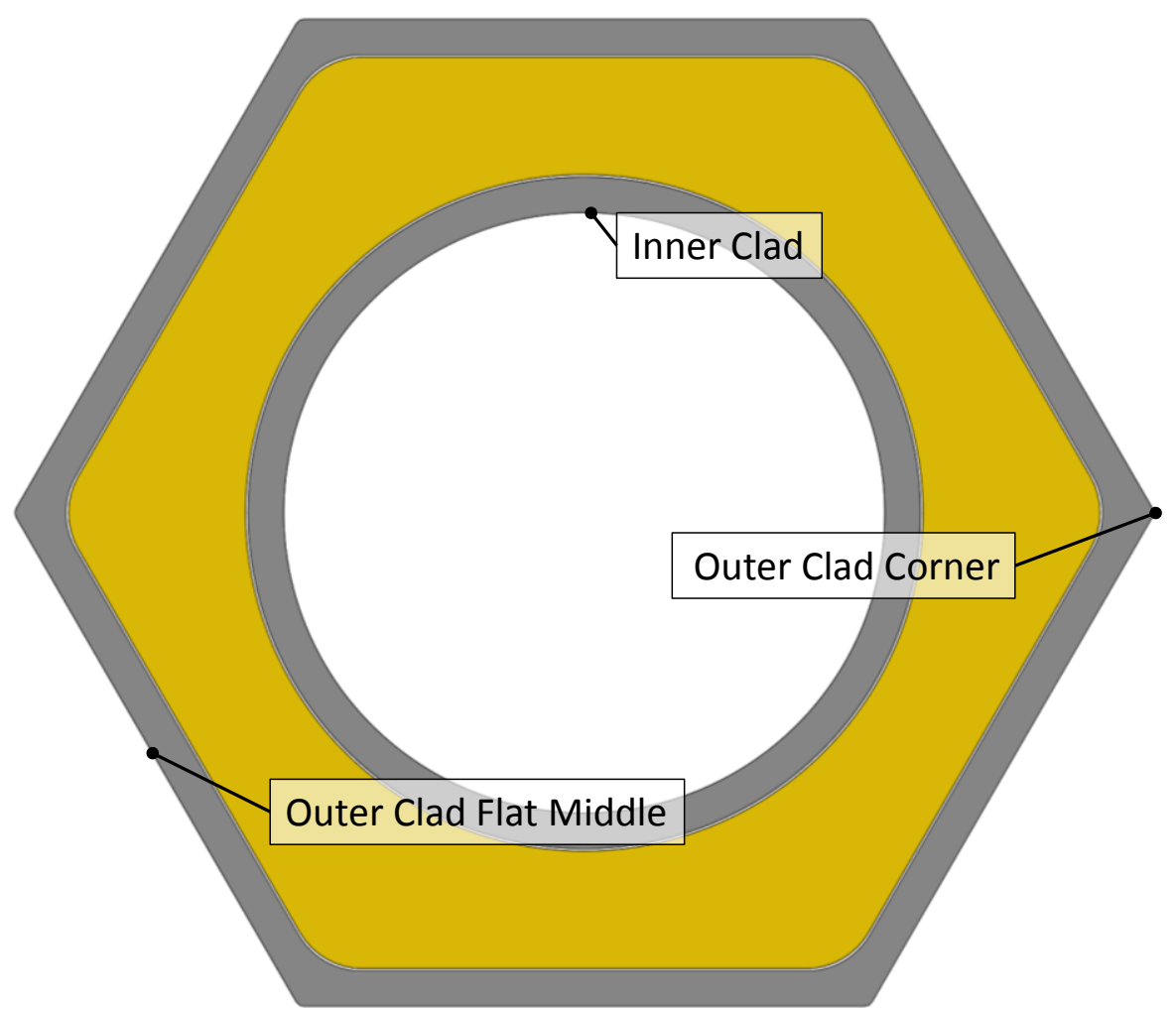

Figure 2.3. Stress result extraction locations 


\subsection{Clad Thickness}

\subsubsection{Temperature Results}

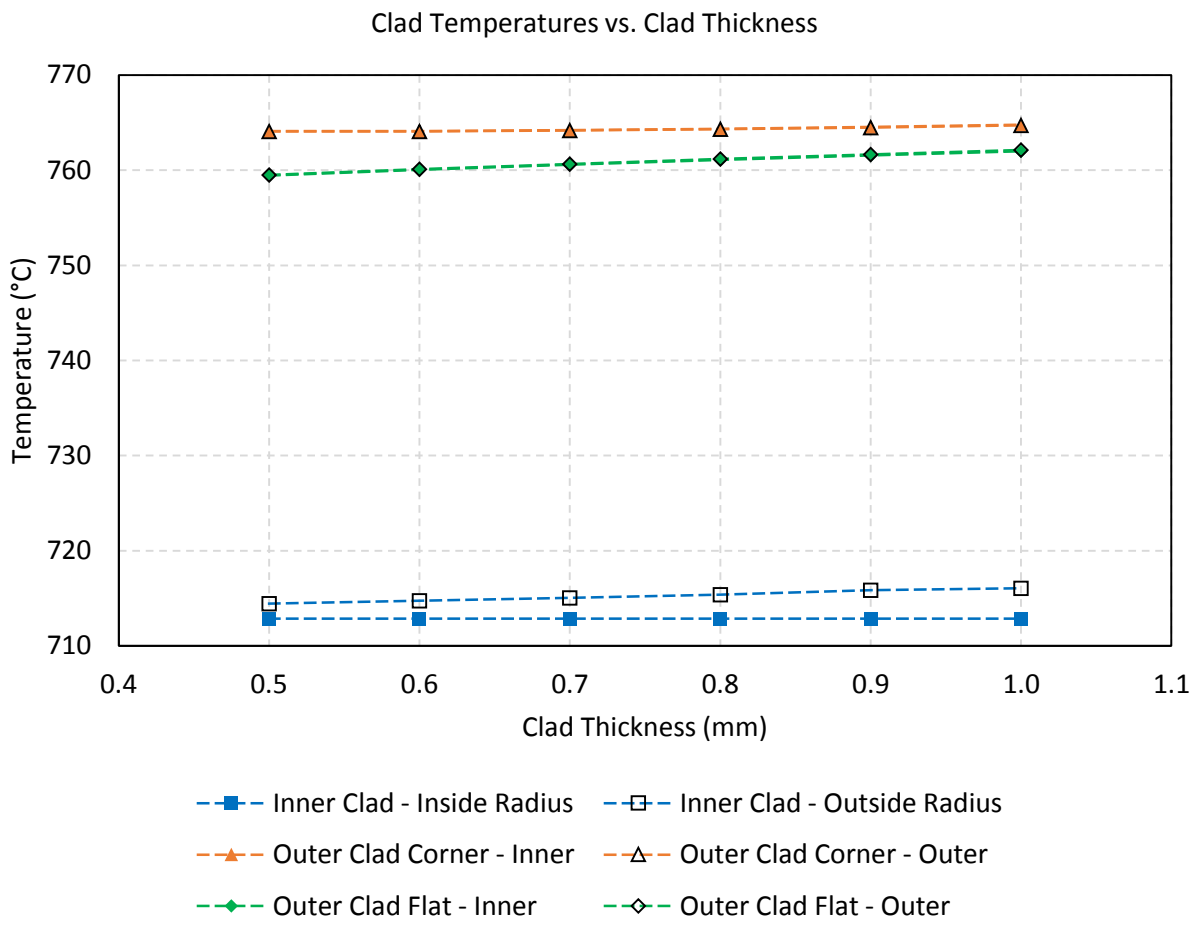

Figure 2.4. Change in clad temperatures as clad thickness is varied

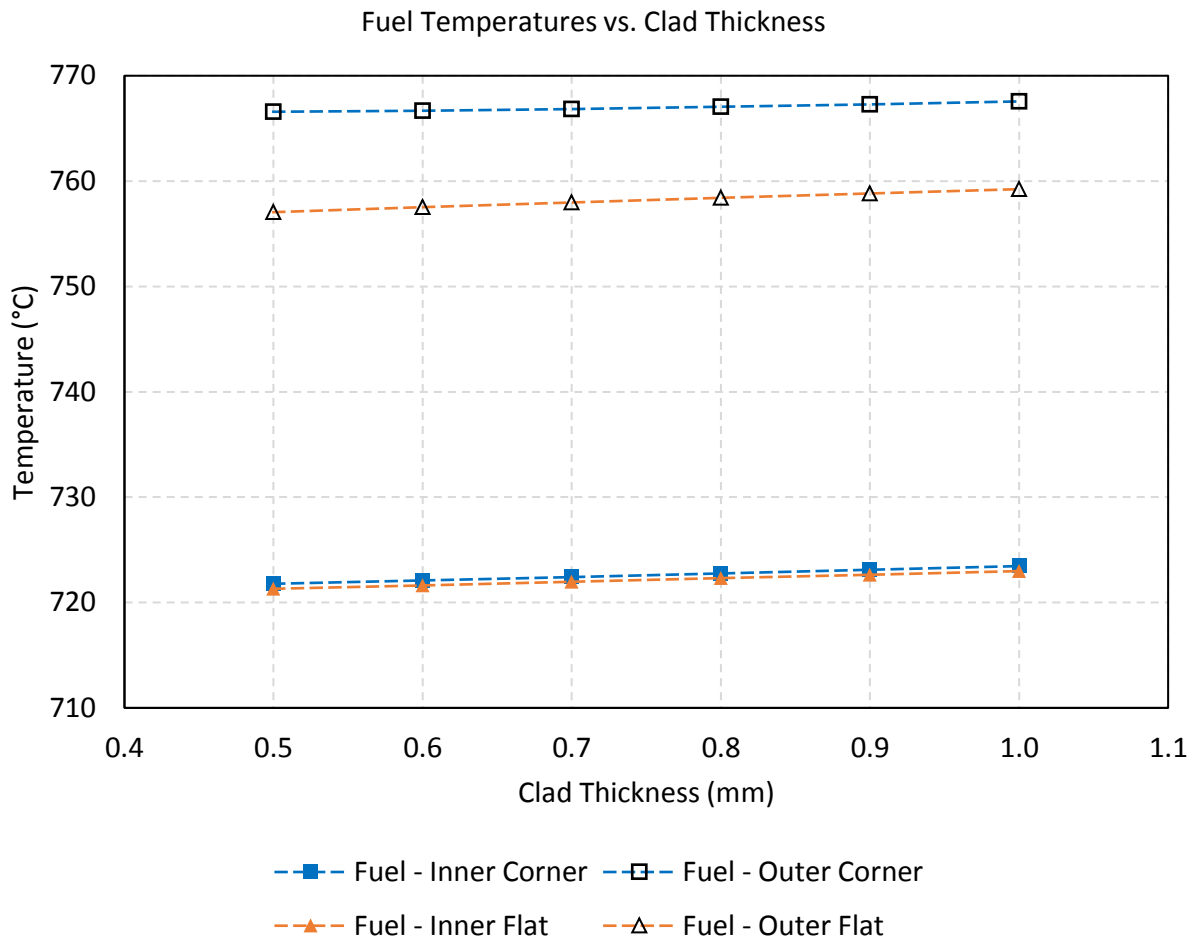

Figure 2.5. Change in fuel temperatures as clad thickness is varied 


\subsubsection{Stress Results}

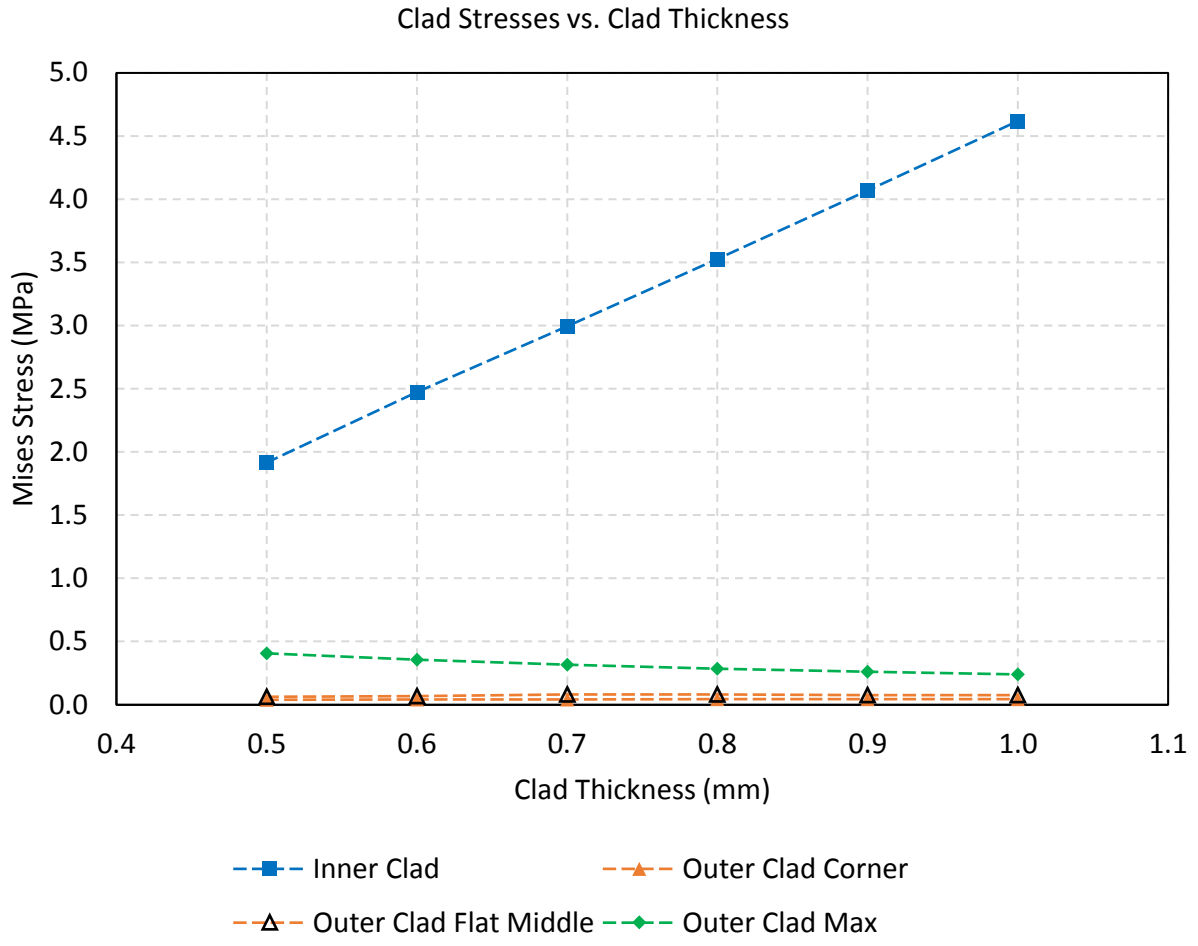

Figure 2.6. Change in clad stress as clad thickness is varied

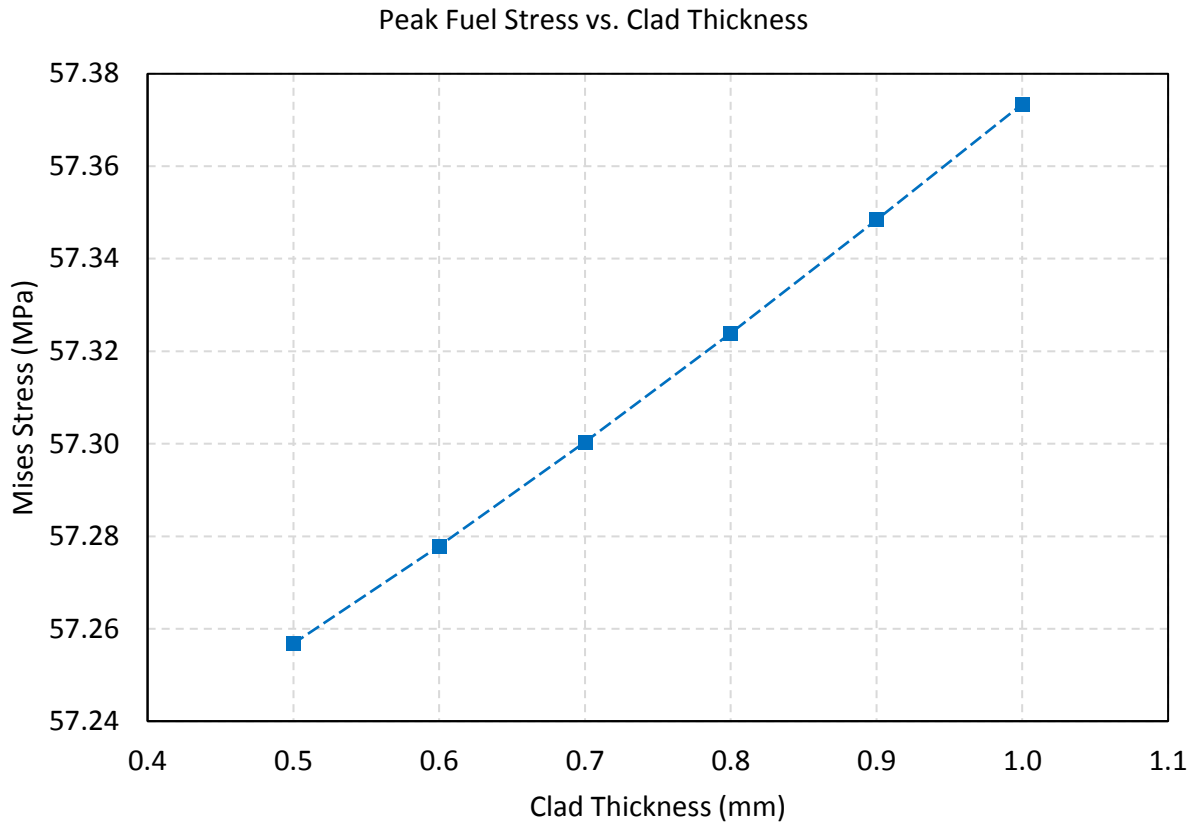

Figure 2.7. Change in peak fuel stress as clad thickness is varied 


\subsection{Inner Helium Gap Thickness}

\subsubsection{Temperature Results}

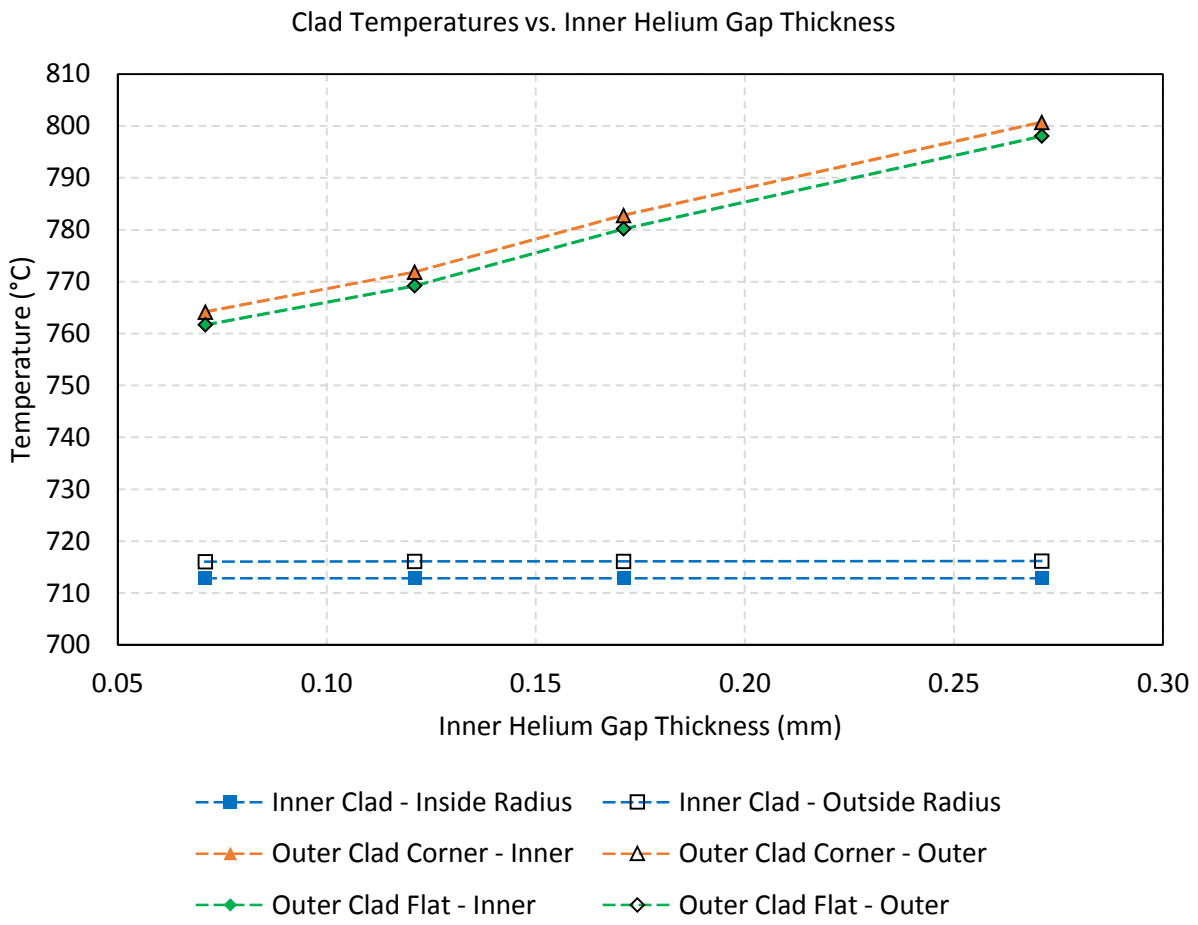

Figure 2.8. Change in clad temperatures as inner helium gap thickness is varied

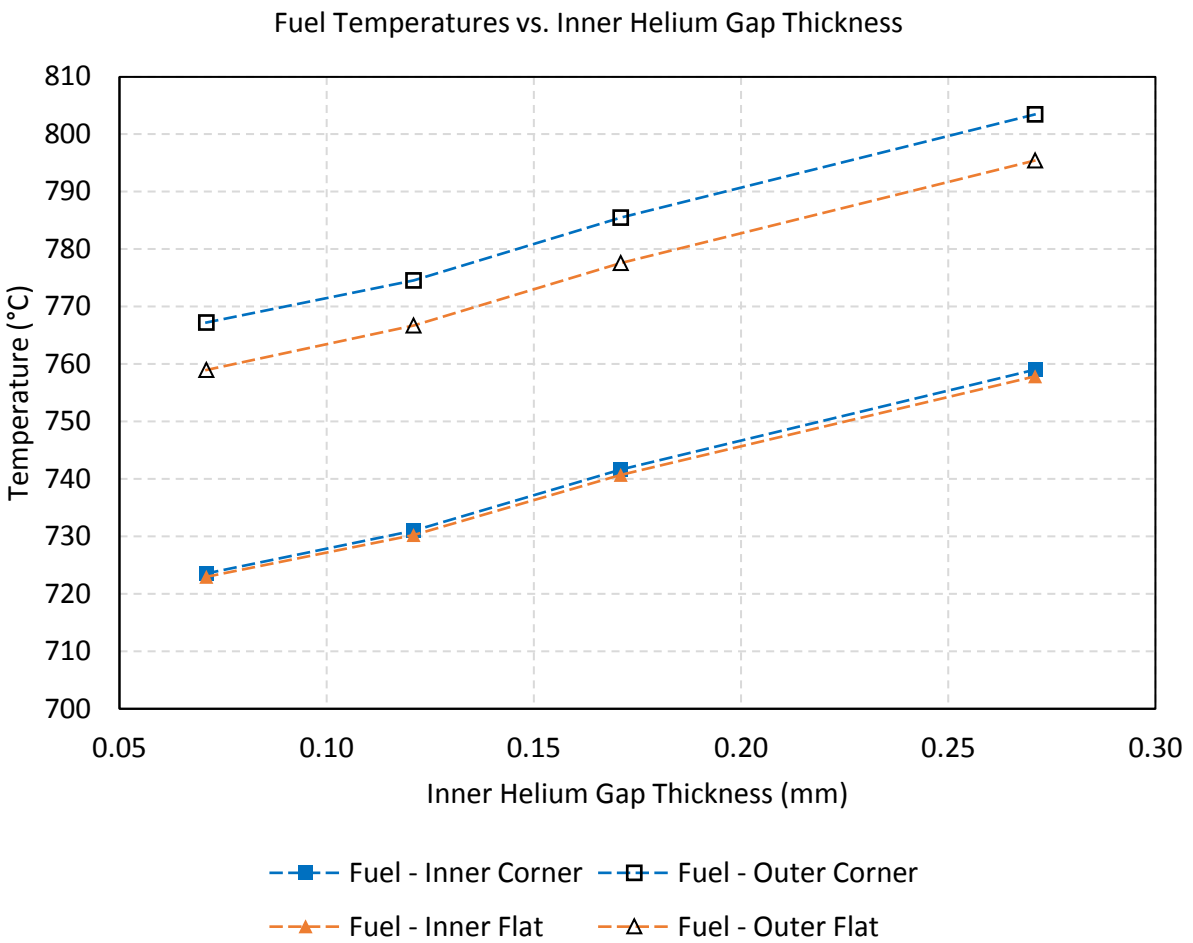

Figure 2.9. Change in fuel temperatures as inner helium gap thickness is varied 


\subsubsection{Stress Results}

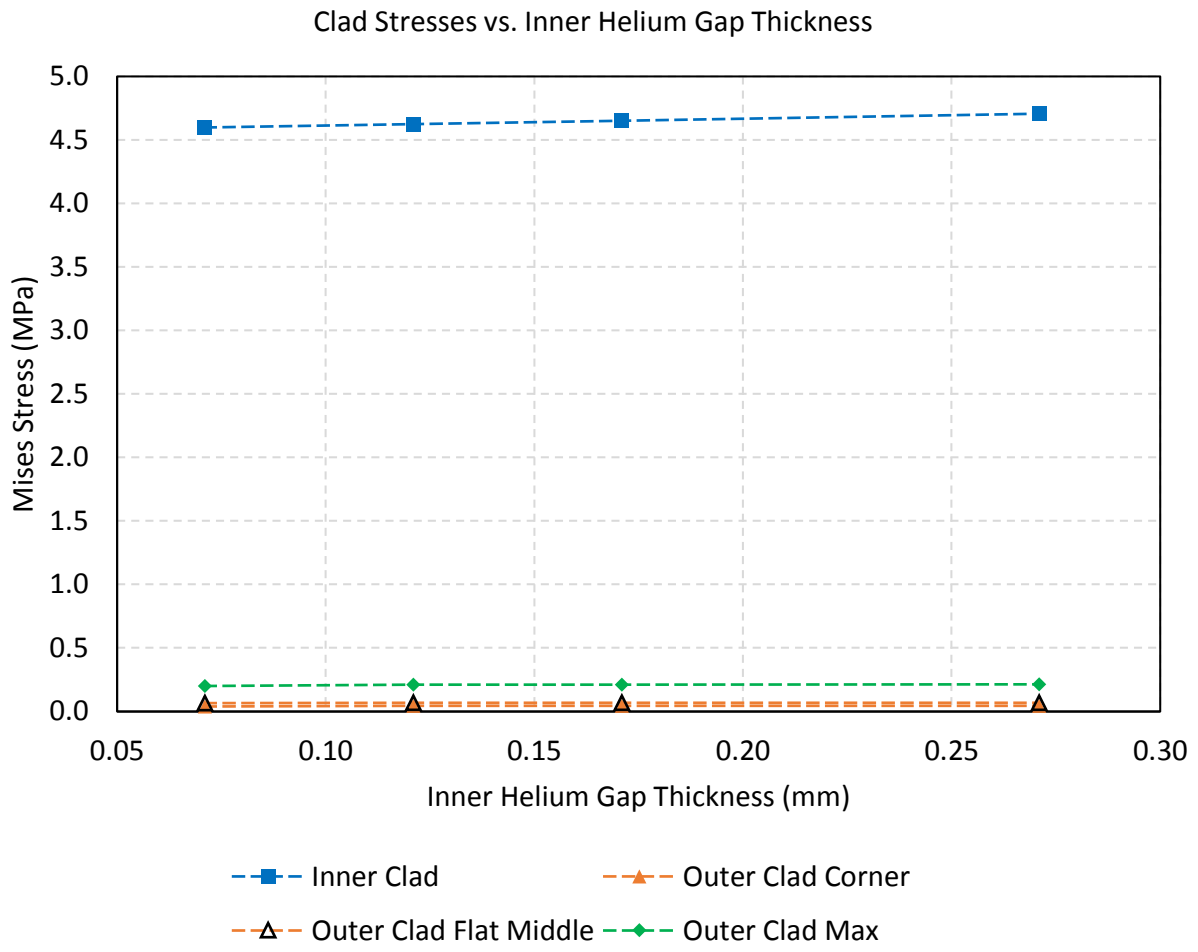

Figure 2.10. Change in clad stress as inner helium gap thickness is varied

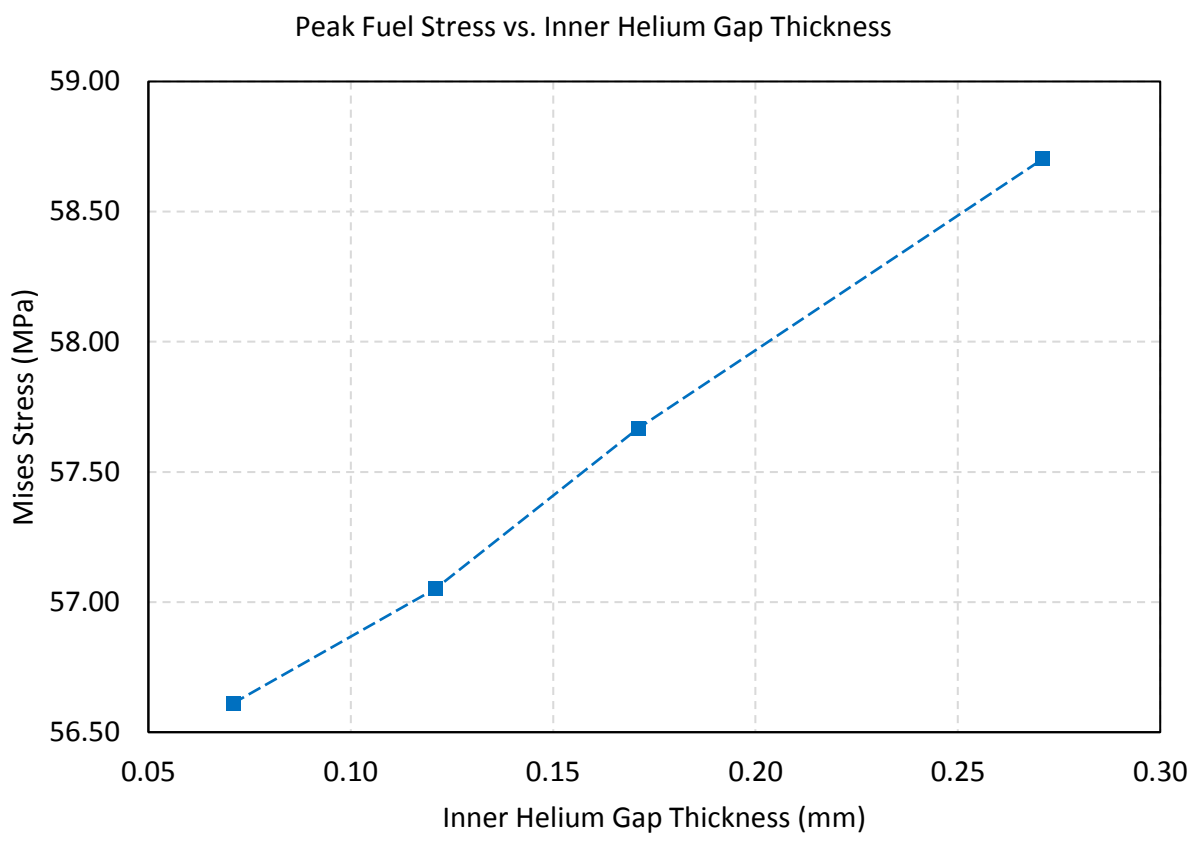

Figure 2.11. Change in peak fuel stress as inner helium gap thickness is varied 


\subsection{Outer-Clad, Inner-Corner Fillet Radius}

\subsubsection{Temperature Results}

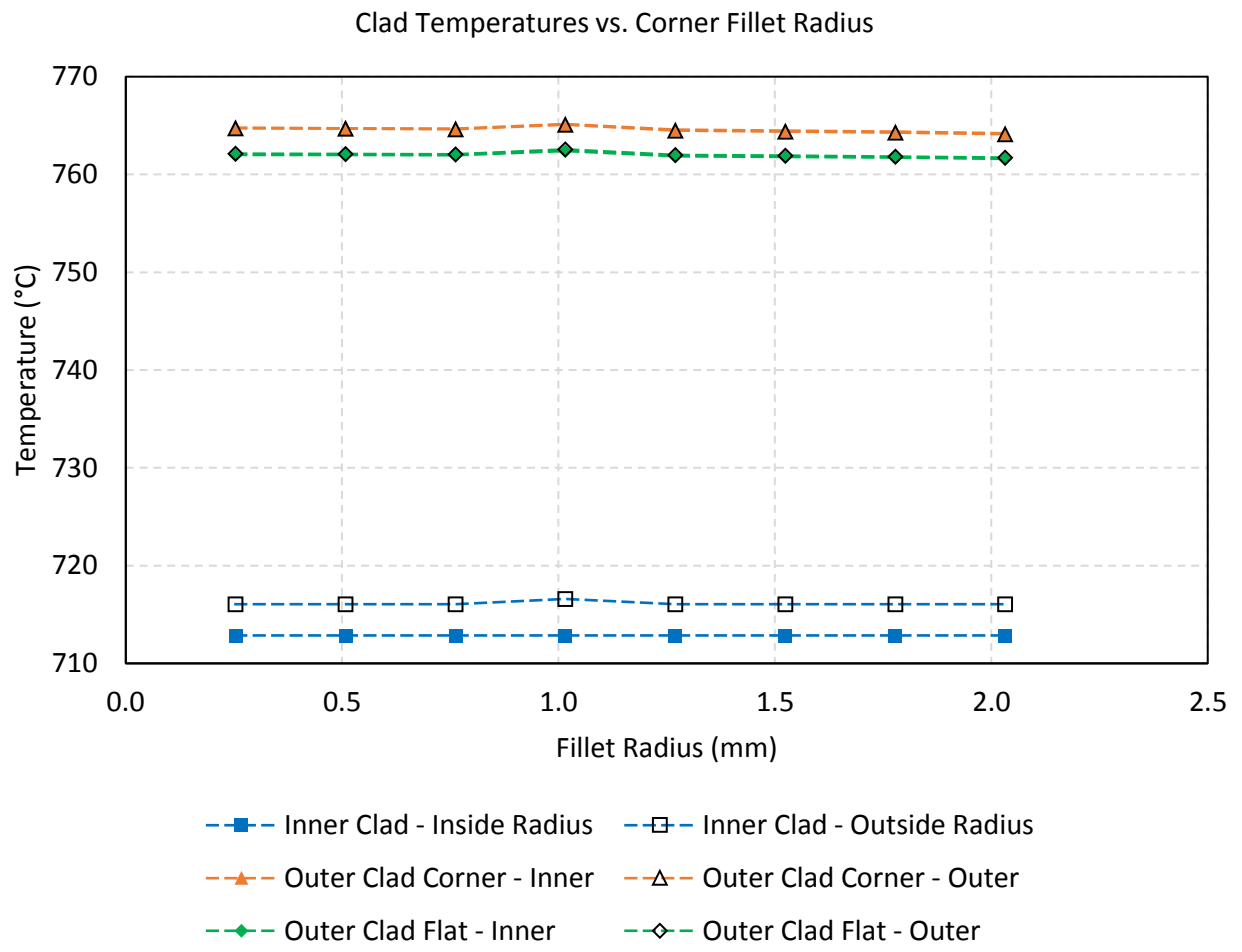

Figure 2.12. Change in clad temperatures as the inner corner fillet radius of the hex clad is varied

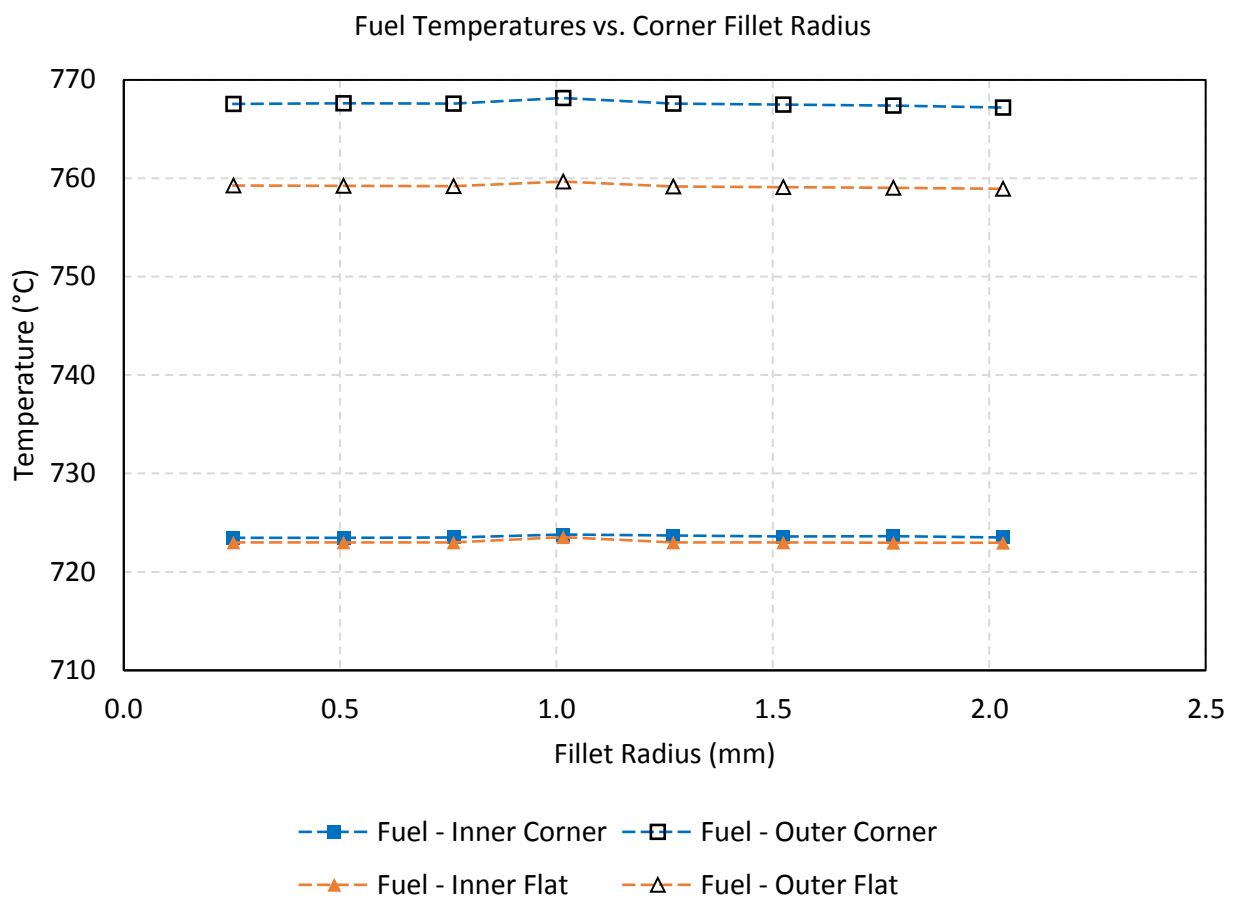

Figure 2.13. Change in fuel temperatures as the inner corner fillet radius of the hex clad is varied 


\subsubsection{Stress Results}

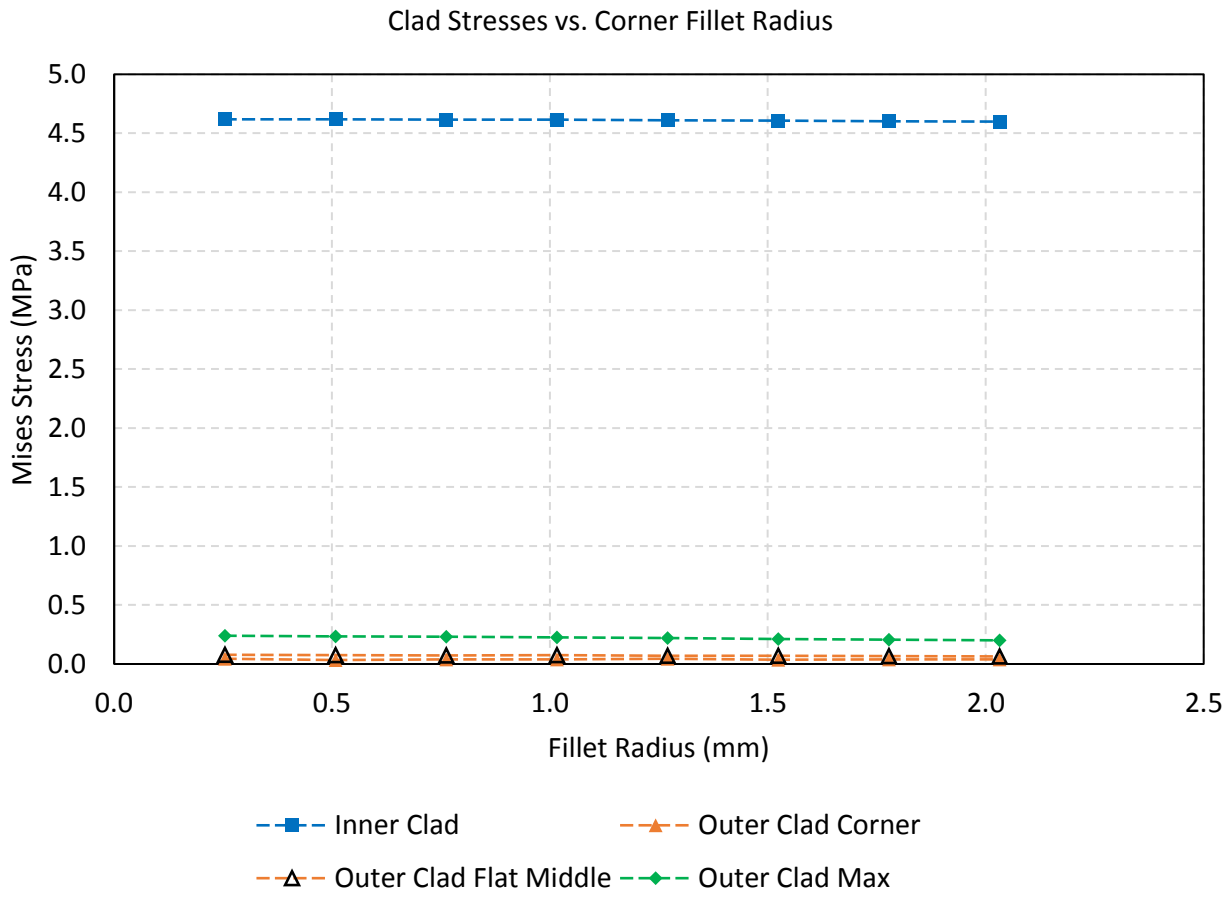

Figure 2.14. Change in clad stress as the inner corner fillet radius of the hex clad is varied

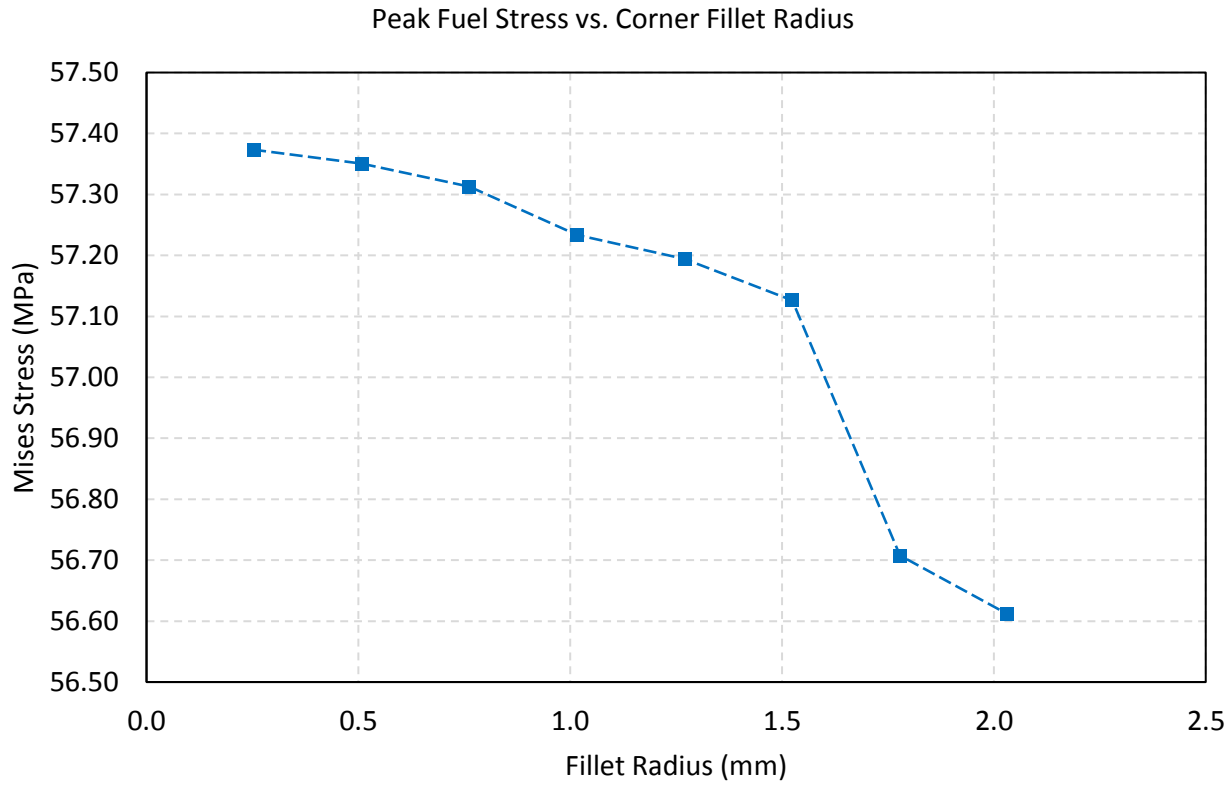

Figure 2.15. Change in peak fuel stress as the inner corner fillet radius of the hex clad is varied 


\subsection{Short-Array Model}

The short-array model is based around the dimensions of Case 8 in Table 2.1. The model consists of seven elements, as shown in Figure 1.2. The results of the uncoupled thermal stress analysis are shown here.

\subsection{Temperature Results}

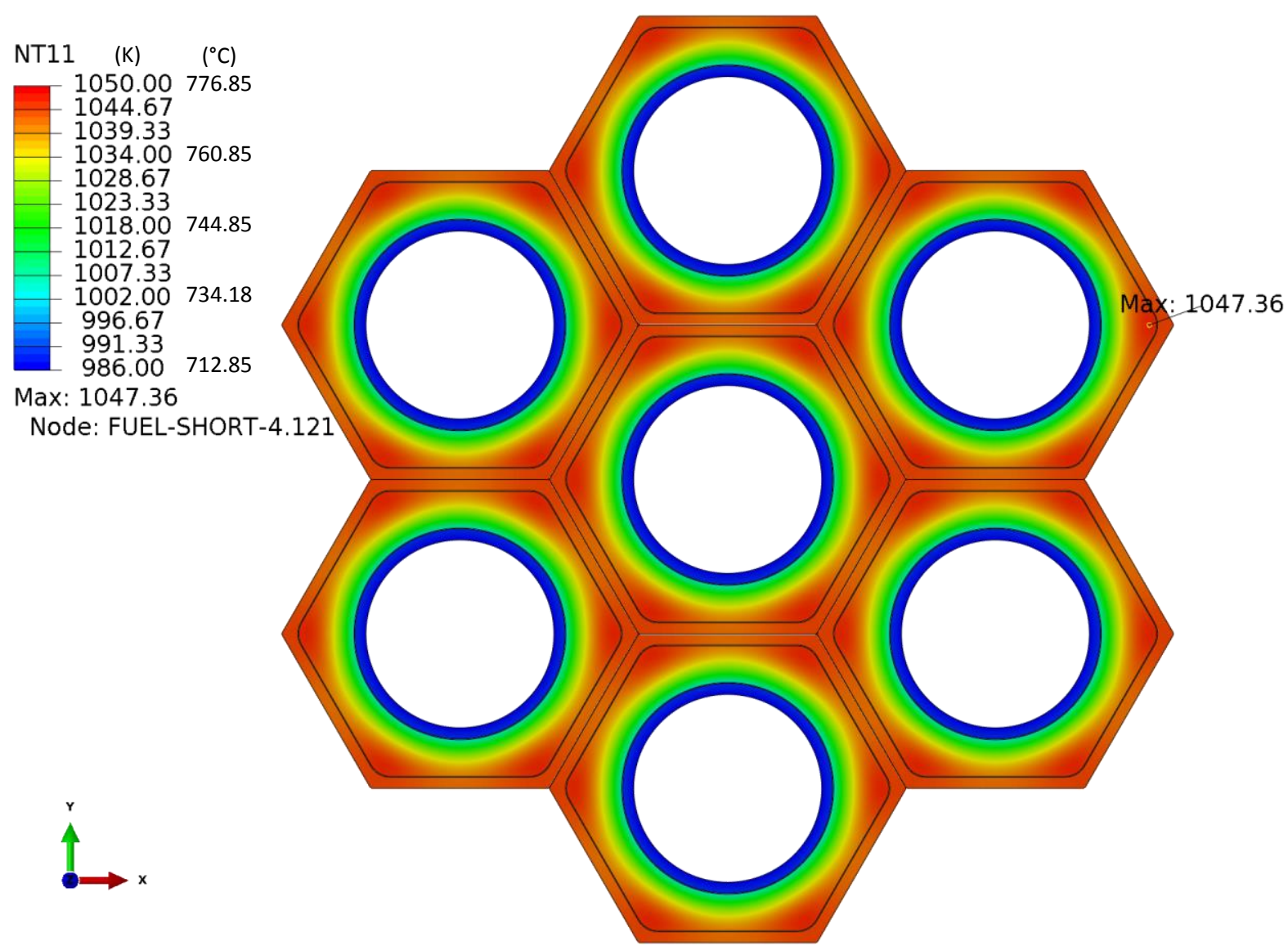

Figure 3.1. Temperature distribution in the short-array model 


\subsection{Full-Length Model}

The full-length model is based around the dimensions of Case 8 in Table 2.1. The model consists of a single full length element. The symmetry boundary conditions have been removed. The fuel is fully encapsulated by cladding, as shown in Figure 1.3. Additionally, the uniform heat generation rate has been replaced by an axially varying rate described by Figure 1.4.

\subsection{Temperature Results}

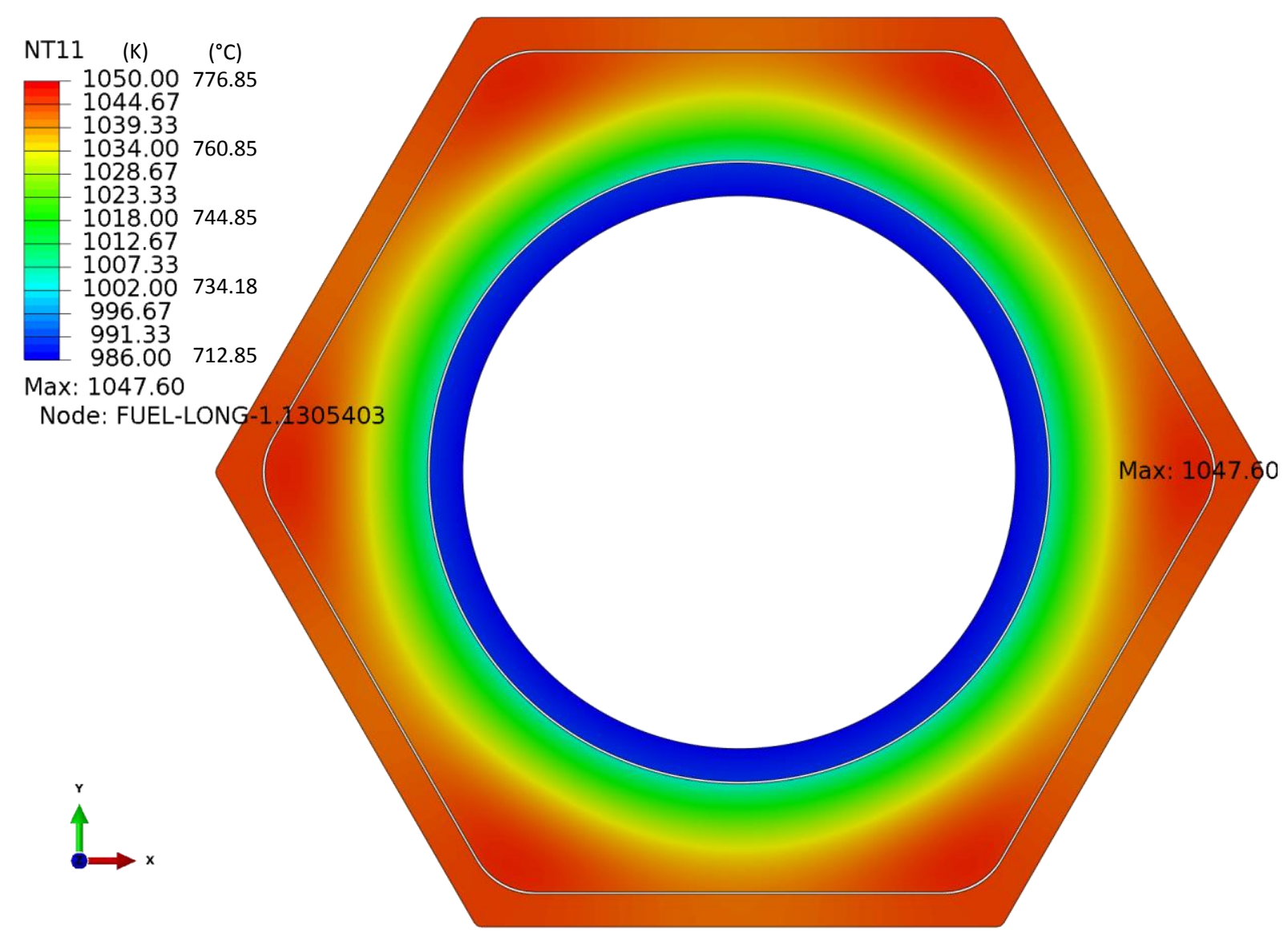

Figure 4.1. X-Y plane temperature distribution halfway along the fuel in the full-length model 

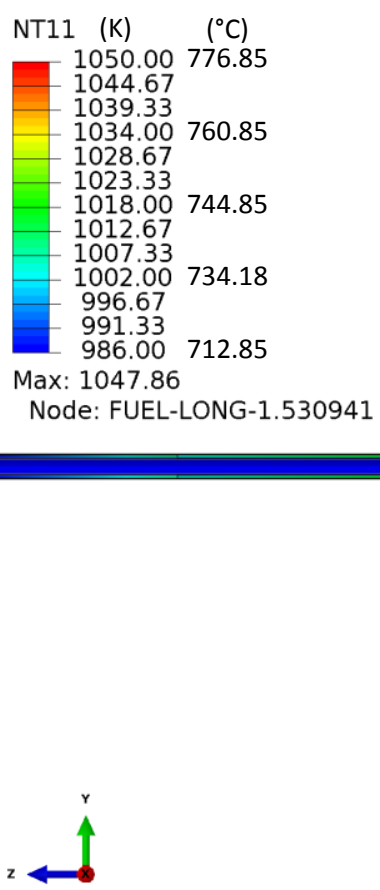

Figure 4.2. Y-Z plane temperature distribution in the full-length model

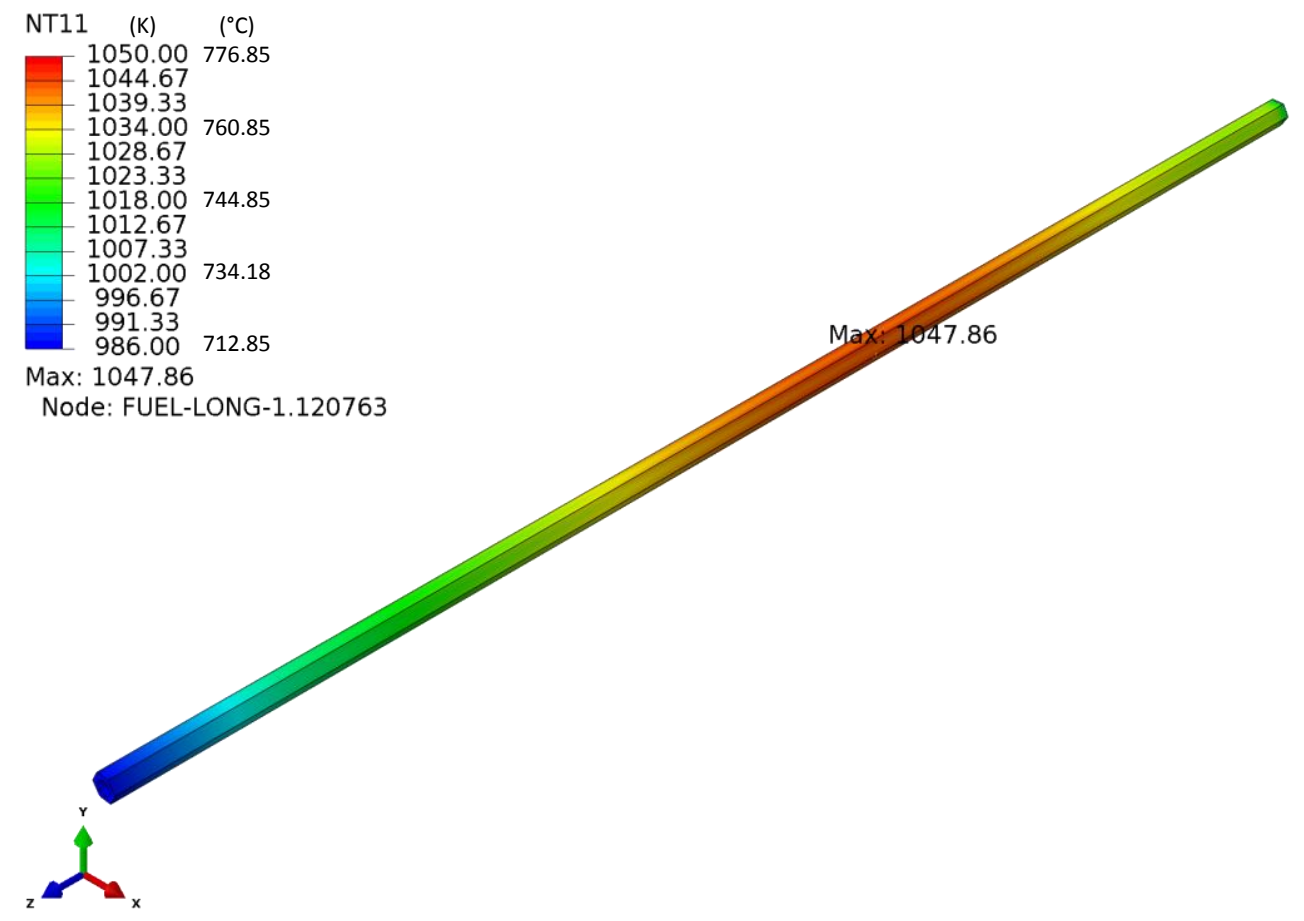

Figure 4.3. Outer clad temperature distribution in the full-length model 


\subsection{Discussion}

\subsection{Thermal Analysis}

A significant advantage of Design A is the largely isothermal temperature distribution in the outer hexagonal cladding. In the inner cladding, a temperature gradient will exist due to the hot fuel on one side, and the cooler heat pipe on the other. Future development work on this design may want to consider techniques for lowering cladding temperatures. With the current iteration, the materials appear to be within acceptable temperature ranges, but with little margin.

\subsection{Stress Analysis}

One of the parametric analyses examined how clad thickness impacts the clad stress. From section 2.1.2, it is clear that an increasing thickness for the inner clad resulted in an increase in the clad stress (Figure 2.6). When calculating the stress through the clad thickness, the temperature difference through the clad is used. As the clad thickness increases, thermal resistance increases through the clad. This results in an increasing temperature differential through the clad as the clad thickness increases. Equation 2, from Timoshenko's Theory of Plates and Shells, shows that clad stress increases as the temperature differential through the clad increases. The equation assumes a linear temperature distribution through the thickness. The stress at the inner surface will be in compression, while the stress at the outer surface is in tension. This is consistent with what is observed in the stress results.

$$
\sigma_{x}=\sigma_{\varphi}= \pm \frac{E \alpha\left(t_{1}-t_{2}\right)}{2(1-v)}
$$

The parametric analysis considered thermal expansion only in the x-y plane. Additionally, there was no variation in temperature in the axial (z) direction. In reality, a fuel element would see axial thermal expansion, leading to elongation of the fuel. Also, there would be a variation in temperature axially, resulting in additional stresses which aren't captured in the 2-D stress calculation of Equation 2. Therefore, when considering a full element or core design, the calculated stresses will increase significantly.

An advantage to Design A is that the stainless steel cladding is subjected primarily to thermal stresses. Within the context of the ASME Boiler and Pressure Vessel Code (BPVC), there are specific rules for thermal stresses and allowable stresses in cladding. Further development of this design should involve a structural engineering evaluation of the allowable stresses as dictated by the ASME BPVC.

\subsection{Conclusion}

The hexagonal element concept demonstrated by Design A appears to be a promising, viable design for a very-small modular reactor. Simplified and fault tolerant manufacturing are among the greatest advantages for this design. Additionally, the stainless steel cladding is dominated by thermal stresses, and it appears that a design which falls within the ASME BPVC is achievable. Future development should consider a structural evaluation of a full core design, and electrically heated, benchtop experiments to validate the numeric analysis. 


\section{Appendix D}

\section{Thermal Analysis -- Design B}

This appendix presents details of a preliminary thermal analysis for Design B. Included in this appendix are descriptions of the computer codes, computer models, and assumptions used to perform the parametric studies in order to evaluate Design B reactor thermal sensitivities and characteristics to help evaluate and support the overall Design B reactor system. 


\section{Design B}

\section{TABLE OF CONTENTS}

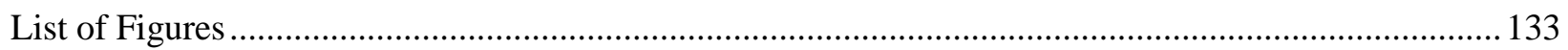

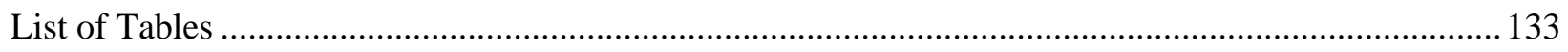

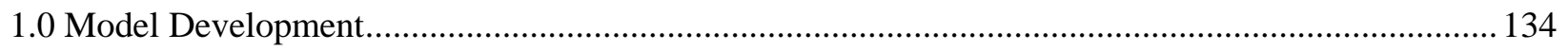

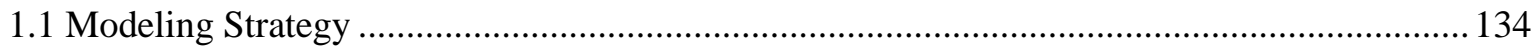

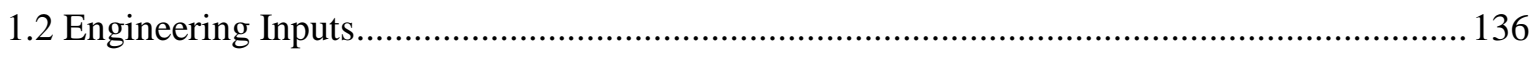

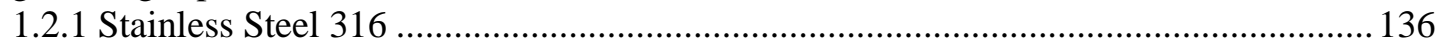

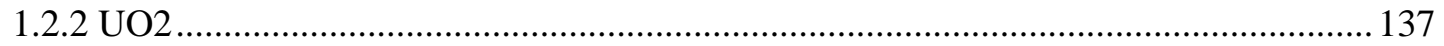

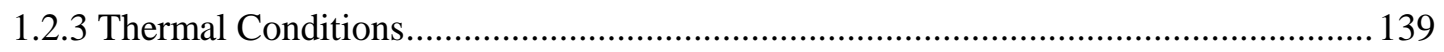

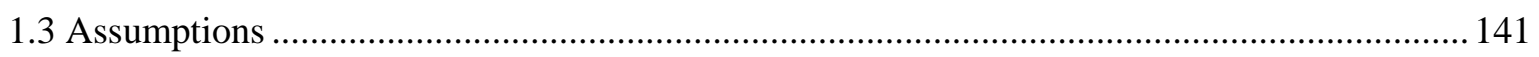

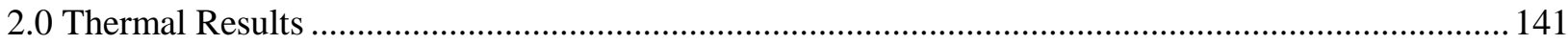

\section{List of Figures}

No table of figures entries found.

\section{List of Tables}

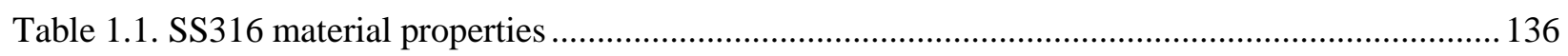

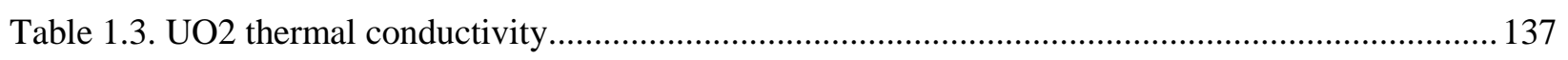

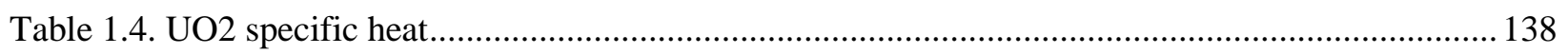




\section{Model Development}

\section{Modeling Strategy}

The model consists of one of the six core segments. There are seven grid plates in the model, resulting in eight sections for sodium. The fuel elements are modeled with the fuel, helium, and cladding all represented. The outer cladding for the heat pipes is also present, with a constant temperature boundary condition on the inner surface of the pipe. Figure 11.1 shows the full model with each of these parts identified. Figure 11.2 shows a cross-section of the model through one of the sodium segments in the core region.

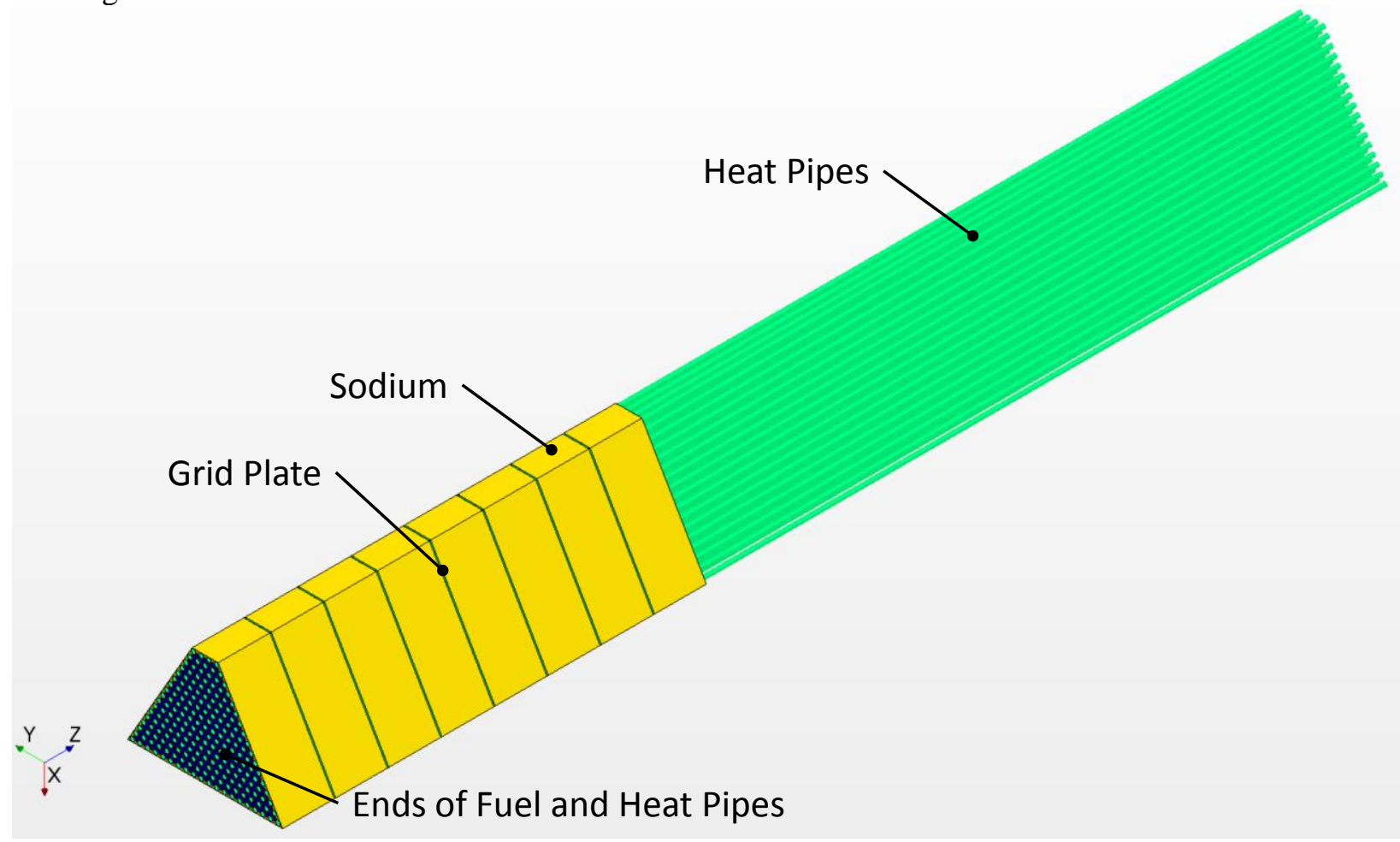

Figure 11.1. Model geometry 


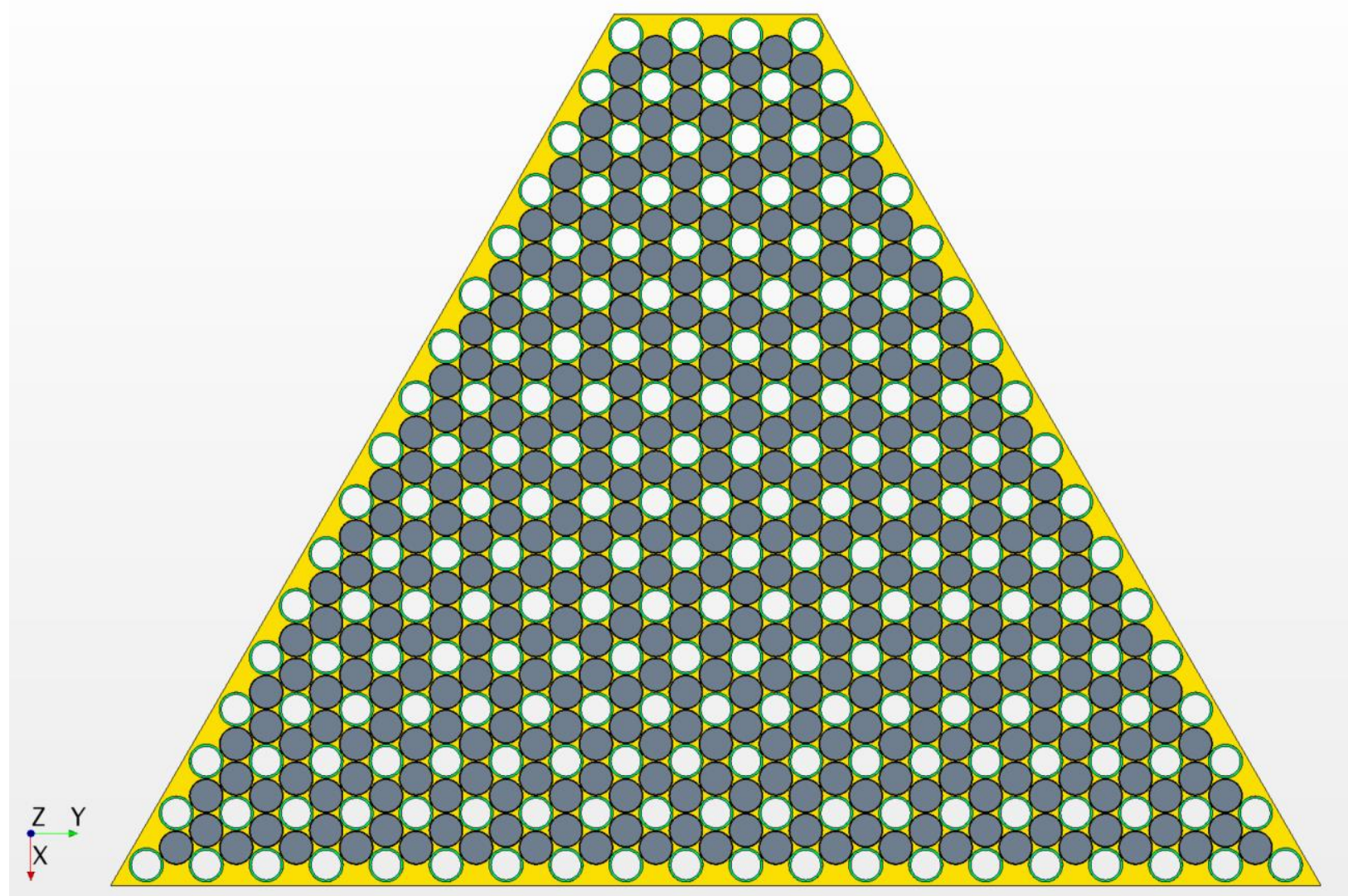

Figure 11.2. Cross-section through sodium core region 


\section{Engineering Inputs}

This section details the material properties and thermal boundary conditions.

\section{Stainless Steel 316}

Stainless steel 316 material properties were specified by selecting the UNSS31600 material in the Star$\mathrm{CCM}+$ physics definition. These values are shown below in Table 11.1.

Table 11.1. SS316 material properties

\begin{tabular}{lcrll}
\hline \multicolumn{1}{c}{ Material Property } & Symbol & Value & & Units \\
\hline Density & $\rho$ & 8000.0 & $\mathrm{~kg} / \mathrm{m}^{3}$ & \\
Specific Heat & $c_{\mathrm{p}}$ & 502.0 & $\mathrm{~J} / \mathrm{kg} \cdot \mathrm{K}$ & \\
Thermal Conductivity & $\mathrm{k}$ & 16.0 & $\mathrm{~W} / \mathrm{m} \cdot \mathrm{K}$ \\
\hline
\end{tabular}




\section{UO2}

\section{Thermal Conductivity}

From: IAEA-TECDOC-1496, "Thermophysical Properties Database of Materials for Light Water Reactors and Heavy Water Reactors, Section 6.1.1.7, Table 1, pp. 91.

A polynomial was fit to the data in Table 1.6 for use in Star-CCM+.

Table 11.2. UO2 thermal conductivity

\begin{tabular}{|c|c|}
\hline $\begin{array}{c}\text { Temperature } \\
\mathbf{T} \\
(\mathbf{K}) \\
\end{array}$ & $\begin{array}{l}\text { Thermal Conductivity }(95 \% \text { Dense }) \\
\mathbf{k} \\
(\mathrm{W} /(\mathbf{m} \cdot \mathbf{K}))\end{array}$ \\
\hline 298.15 & 7.61 \\
\hline 300 & 7.59 \\
\hline 400 & 6.58 \\
\hline 500 & 5.78 \\
\hline 600 & 5.14 \\
\hline 700 & 4.61 \\
\hline 800 & 4.17 \\
\hline 900 & 3.79 \\
\hline 1000 & 3.47 \\
\hline 1100 & 3.19 \\
\hline 1200 & 2.95 \\
\hline 1300 & 2.74 \\
\hline 1400 & 2.56 \\
\hline 1500 & 2.41 \\
\hline 1600 & 2.29 \\
\hline 1700 & 2.19 \\
\hline 1800 & 2.12 \\
\hline 1900 & 2.08 \\
\hline 2000 & 2.06 \\
\hline 2100 & 2.07 \\
\hline 2200 & 2.09 \\
\hline 2300 & 2.14 \\
\hline 2400 & 2.20 \\
\hline 2500 & 2.28 \\
\hline 2600 & 2.37 \\
\hline 2700 & 2.48 \\
\hline 2800 & 2.59 \\
\hline 2900 & 2.71 \\
\hline 3000 & 2.84 \\
\hline 3100 & 2.97 \\
\hline 3120 & 2.99 \\
\hline
\end{tabular}




\section{Density}

From: IAEA-TECDOC-1496, "Thermophysical Properties Database of Materials for Light Water Reactors and Heavy Water Reactors," Section 6.1.1.10, Table 1, pp. 115

Density:

$$
\rho=10760 \mathrm{~kg} / \mathrm{m}^{3}
$$

\section{Specific Heat}

From: IAEA-TECDOC-1496, "Thermophysical Properties Database of Materials for Light Water

Reactors and Heavy Water Reactors," Section 6.1.1.1, Table 3, pp. 29

A polynomial was fit to the data in Table 1.7 for use in Star-CCM+

Table 11.3. UO2 specific heat

\begin{tabular}{|c|c|}
\hline $\begin{array}{c}\mathbf{T} \\
(\mathbf{K}) \\
298.15\end{array}$ & $\begin{array}{c}\mathbf{C}_{\mathbf{p}} \\
(\mathbf{J} / \mathbf{k g} \cdot \mathbf{K}) \\
235\end{array}$ \\
\hline 300 & 235 \\
\hline 400 & 266 \\
\hline 500 & 282 \\
\hline 600 & 292 \\
\hline 700 & 299 \\
\hline 800 & 304 \\
\hline 900 & 308 \\
\hline 1000 & 312 \\
\hline 1100 & 315 \\
\hline 1200 & 318 \\
\hline 1300 & 320 \\
\hline 1400 & 324 \\
\hline 1500 & 327 \\
\hline 1600 & 332 \\
\hline 1700 & 339 \\
\hline 1800 & 347 \\
\hline 1900 & 358 \\
\hline 2000 & 373 \\
\hline 2100 & 390 \\
\hline 2200 & 411 \\
\hline 2300 & 437 \\
\hline 2400 & 466 \\
\hline 2500 & 500 \\
\hline 2600 & 537 \\
\hline 2700 & 579 \\
\hline 2800 & 625 \\
\hline 2900 & 674 \\
\hline 3000 & 726 \\
\hline 3100 & 781 \\
\hline 3120 & 792 \\
\hline
\end{tabular}




\section{Sodium}

\section{Density}

Sodium density is specified as a constant value.

$\rho=802.0 \mathrm{~kg} / \mathrm{m}^{3}$

\section{Dynamic Viscosity}

From: Faghri, A., Heat Pipe Science and Technology, Second Editions, Global Digital Press, 2016, pp. 897, 908.

A polynomial was fit to the data in Table 11.4 for use in Star-CCM+ Table 11.4. Sodium dynamic viscosity

\begin{tabular}{cc}
\hline Temperature & Liquid Viscosity \\
$\mathbf{T}$ & $\boldsymbol{\mu}_{\mathbf{1}}$ \\
$(\mathbf{K})$ & $(\mathbf{P a} \cdot \mathbf{s})$ \\
\hline 600 & 0.0003276 \\
700 & 0.000269 \\
800 & 0.0002298 \\
900 & 0.0002018 \\
1000 & 0.0001809 \\
1100 & 0.0001645 \\
1200 & 0.0001514 \\
1300 & 0.0001407 \\
1400 & 0.0001317 \\
1500 & 0.000124 \\
1600 & 0.0001176 \\
1700 & 0.0001117 \\
1800 & 0.0001067 \\
\hline
\end{tabular}

\section{Specific Heat}

Specific heat is specified as a constant value. $\mathrm{c}_{\mathrm{p}}=1260.0 \mathrm{~J} / \mathrm{kg} \cdot \mathrm{K}$ 


\section{Thermal Conductivity}

From: Faghri, A., Heat Pipe Science and Technology, Second Editions, Global Digital Press, 2016, pp. 897, 908. A polynomial was fit to the data for use in Star-CCM+.

Table 11.5. Sodium thermal conductivity

\begin{tabular}{cc}
\hline Temperature & Liquid Thermal Conductivity \\
$\mathbf{T}$ & $\mathbf{k}$ \\
$(\mathbf{K})$ & $(\mathbf{W} / \mathbf{m} \cdot \mathbf{K})$ \\
\hline 600 & 75.17 \\
700 & 70.53 \\
800 & 65.88 \\
900 & 61.25 \\
1000 & 56.6 \\
1100 & 51.96 \\
1200 & 47 \\
1300 & 42.5 \\
1400 & 37.5 \\
1500 & 33 \\
1600 & 28.5 \\
1700 & 24 \\
1800 & 19 \\
\hline
\end{tabular}

\section{Helium}

Helium material properties were specified by selecting the He material in Star-CCM+. These values are shown below in Table 11.6.

Table 11.6. Helium material properties

\begin{tabular}{lcrll}
\hline \multicolumn{1}{c}{ Material Property } & Symbol & Value & \multicolumn{1}{c}{ Units } \\
\hline Dynamic Viscosity & $\mu$ & $1.9891 \cdot 10^{-5}$ & $\mathrm{~Pa} \cdot \mathrm{s}$ & \\
Molecular Weight & $\mathrm{N}$ & 4.0026 & $\mathrm{~kg} / \mathrm{kmol}$ & \\
Specific Heat & $\mathrm{c}_{\mathrm{p}}$ & 5197.61 & $\mathrm{~J} / \mathrm{kg} \cdot \mathrm{K}$ & \\
Thermal Conductivity & $\mathrm{k}$ & 0.154933 & $\mathrm{~W} / \mathrm{m} \cdot \mathrm{K}$ & \\
\hline
\end{tabular}

\section{Thermal Conditions}

To simplify the initial analysis, a uniform heat generation rate was assumed for all fuel in the model. This number was found by taking the total core thermal power $(5 \mathrm{MW})$ and dividing by the number of segments (6). This resulted in a total power of $0.83 \mathrm{MW}$ for all fuel in the model.

The inner surface of the heat pipe has a constant temperature boundary condition of $713^{\circ} \mathrm{C}(986 \mathrm{~K})$. This value is a reasonable approximation from the LANL HTPIPE code. 


\section{Assumptions}

- The model is assumed to be steady state (no transient events are considered)

- The sodium and helium are assumed stagnant (no fluid motion). Therefore, natural convection and buoyancy forces from sodium motion are not accounted for.

- Only a basic thermal solution is presented - no structural analysis has yet been completed.

\section{Thermal Results}

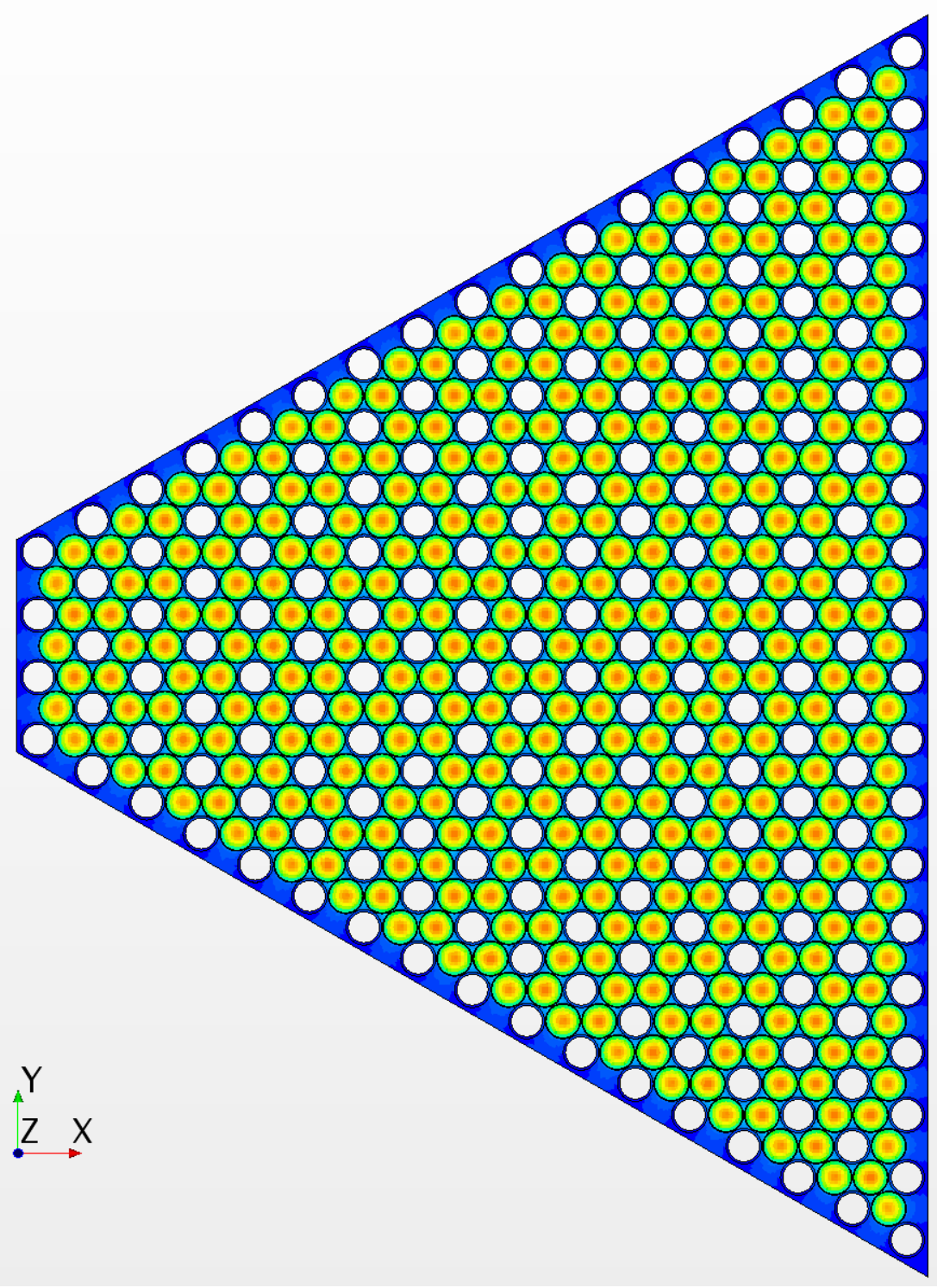

Temperature (C)

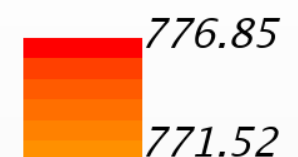

766.18

760.85

755.52

750.18

744.85

739.52

734.18

728.85

723.52

718.18

712.85

Figure 12.1. X-Y plane temperature cross-section 


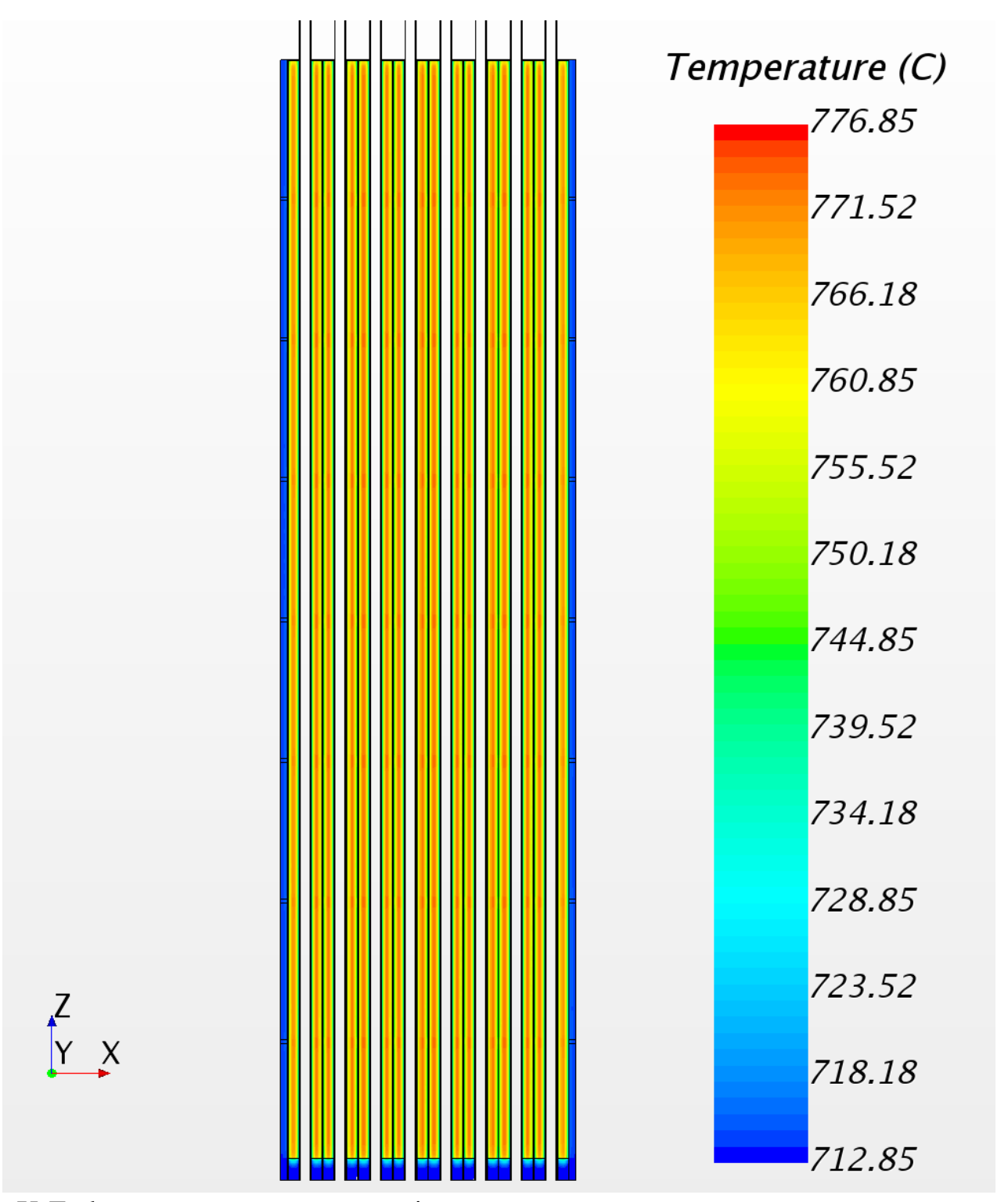

Figure 12.2. X-Z plane temperature cross-section 


\section{Appendix E}

\section{Steady-State Simulation of Liquid Metal Heat Pipes}

This appendix presents the heat pipe performance analysis for both Design A and Design B. The same heat pipe design is used both concepts. Included are descriptions of the computer codes, computer models, and assumptions used to perform the verification, validation, parametric studies, and evaluation of the heat pipe design to provide primary core cooling for a small modular fast reactor. 


\section{CONTENTS}

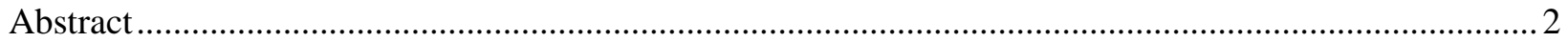

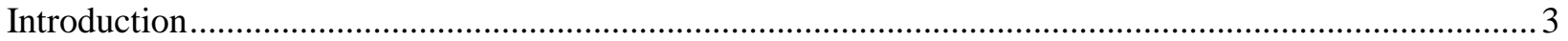

What is a heat pipe

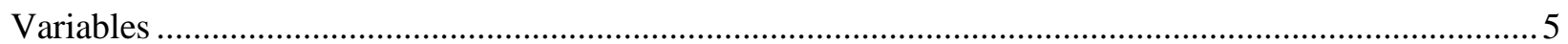

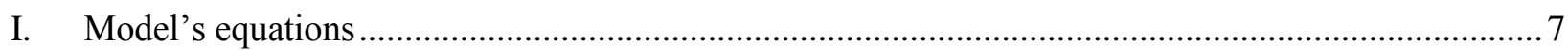

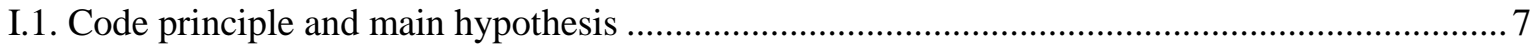

I.2. Incompressible one-dimensional vapor flow ….............................................................. 7

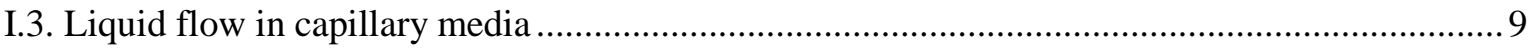

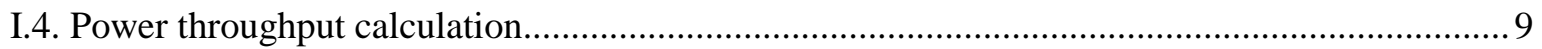

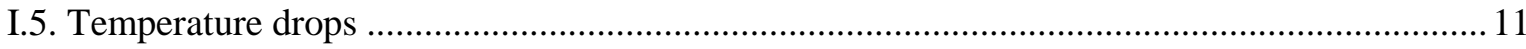

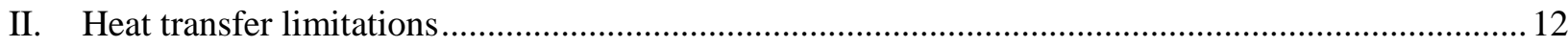

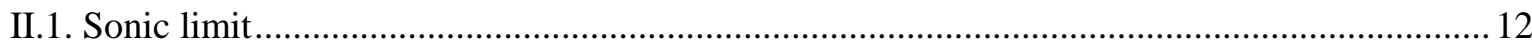

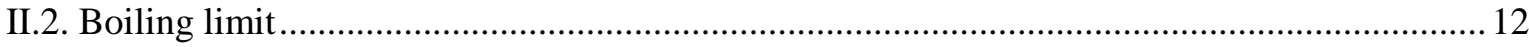

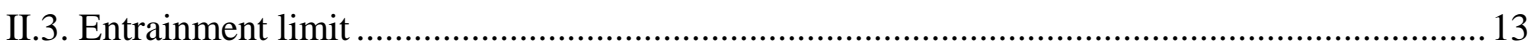

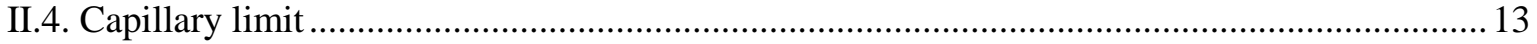

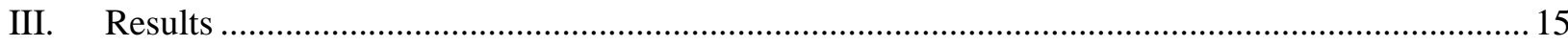

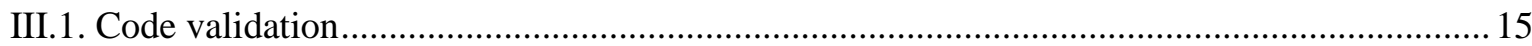

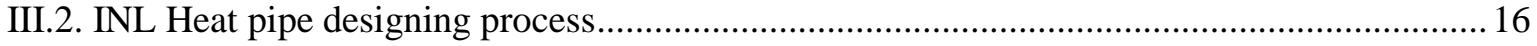

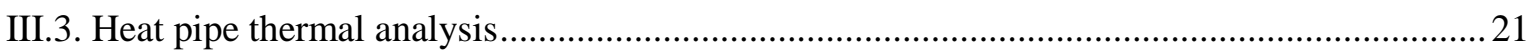

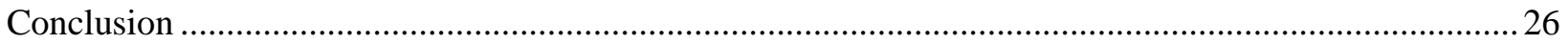

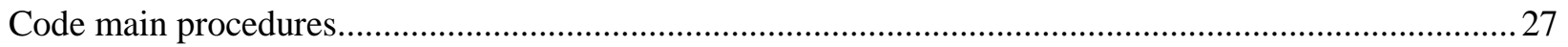

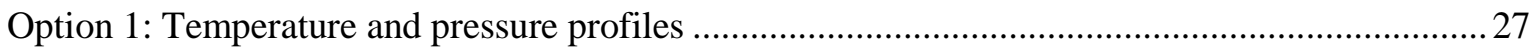

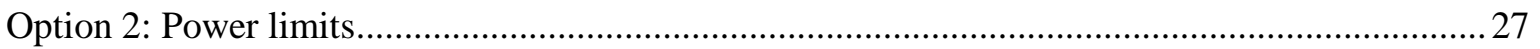

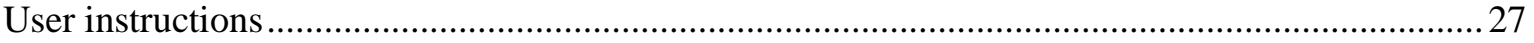

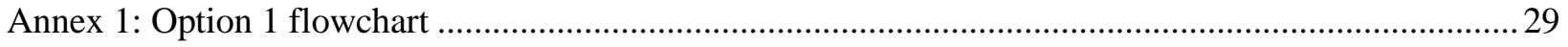

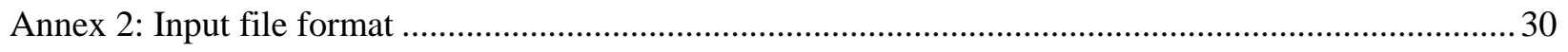

Annex 3: Heat transfer with source, example of input file ….............................................................. 31

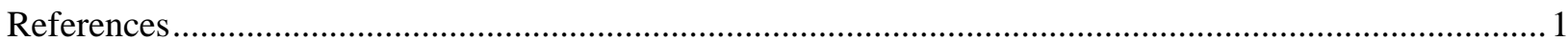




\begin{abstract}
Heat-pipe cooled fast reactors have been identified as serious candidates for providing durable and safe energy to remote locations isolated from stable electrical grids. The Specific Purpose Reactor currently in development in the Los Alamos and Idaho National Laboratories should produce about $2 \mathrm{MWe}$ for strategic defense or emergency locations.

A steady-state simulation code was programmed to evaluate the performances of liquid metal heat pipes as the primary cooling system of a 5MWth fast reactor. The model uses simple first order fluid mechanic concepts, neglecting some complex phenomena, to determine the power limitations of common types of heat pipes. A thermal analysis procedure was also implemented, giving access to temperature profiles of the working fluid and the different layers of the input pipe.

Once the model was implemented and compared to results found in the literature, several designs compatible with the reactor geometry were simulated. Screened annular gap appeared to be the best available option but gave only small power margins under operating conditions. Satisfying results are obtained by changing slightly the initial reactor design or with innovative heat pipes still in development at Los Alamos National Laboratory. The next step will be the manufacture of prototypes and the experimental tests on heat pipes.
\end{abstract}




\section{Introduction}

The present work was conducted in the framework of the Specific Purpose Mega-Power Reactor project. This fission reactor, developed in cooperation between LANL and INL, is supposed to provide between 1 and 2 MWe of electricity (with a total power of approximately 5MWth) for remote sites isolated from electrical grids. The use of liquid metal heat pipes is investigated as the primary cooling system, for its safety and versatility.

The thermodynamics of heat pipes have been the subject of considerable literature and its simulation in three dimensions remains a tremendous challenge. This document will detail a simple model aiming at finding the operating temperature and pressure of a heat pipe under a specific set of conditions, corresponding to our project needs. This model is inspired of a previous code developed by LANL (Los Alamos National Laboratory) researcher Keith A. Woloshun in 1988. The simulation was coded at the time in Fortran 77 and needed to be translated into python, both for integration into wider applications and for specific modifications purposes. In particular, the new code should enable a more precise discretization of the heat pipe and take into account a more realistic (regarding this project) heat transfer both between the source and the pipe and between the pipe and the sink.

\subsection{What is a heat pipe?}

A heat pipe is a metallic pipe filled with a working fluid at equilibrium between its liquid and vapor phases. The inside of the pipe wall is generally covered by a porous structure called wick, enabling liquid flow by capillarity while the center is an open channel for vapor flow. A heat pipe is divided into two main sections: the evaporator section is in contact with the heat source (in this case the fuel rods) and evacuate the heat by evaporation of the liquid phase. The vapor created flows towards the end of the pipe and transfers its latent heat to the sink as it turns back into the liquid phase. The condensation occurs in the condenser section, in contact with the secondary cooling system. The liquid is then pumped back to the evaporator by capillarity effect through the porous wick. A third section, called the adiabatic section, can exist if the source is not in contact with the sink. In this case, the vapor and the liquid flow through this section without any heat exchange and only undergo viscous loss, compensated by the increase of the fluid's velocity.

Figure C-29 | Schematic view of a heat pipe. Liquid in a saturated wick evaporates in contact with the heat source and condensates after flowing to the condenser section, transferring its latent heat to the sink. The liquid flows back to the evaporator by capillarity.

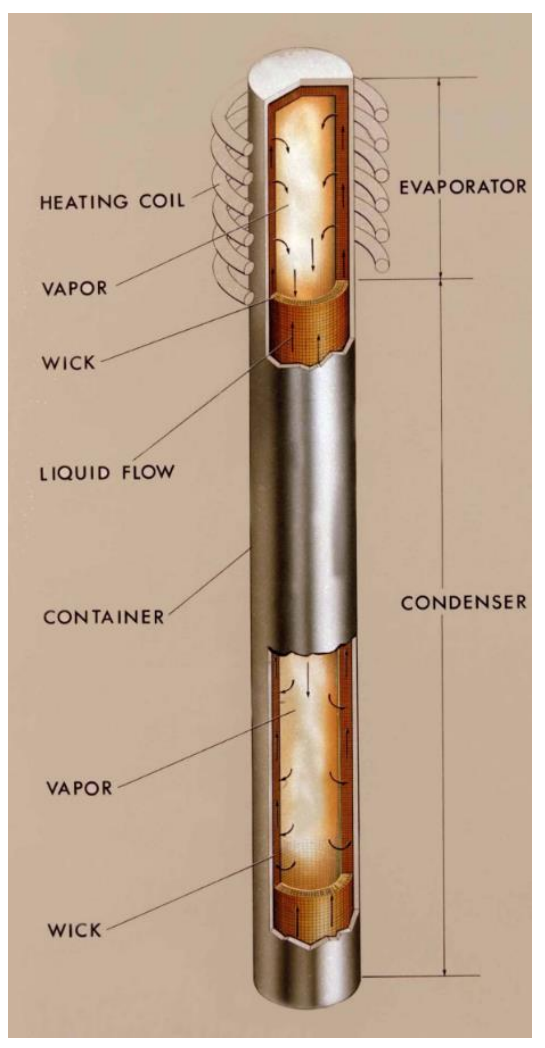

Source: DOE report LA-UR-15-28840

Heat pipes present many advantages. First of all, it is a passive system that uses very small amounts of working fluid, which reduces significantly the risks of major accidents. Being all independents and used in large numbers, the probability of primary cooling complete failure is 
extremely low, and in the case of an accident, the emergency cooling of the reactor will be passively assured to a certain extent. Similarly, the loss due to a leak will be low, and the consequent risks will be reduced given the low operating pressure of heat pipes (typically less than one atmosphere). The thermodynamic properties of heat pipes are its other huge advantage. All the heat being exchanged by phase change, the heat pipe is almost isothermal once steady-state is reached. Besides, it can operate at any temperature between $-270 \mathrm{~K}$ and $2000 \mathrm{~K}$, depending on the working fluid, the cladding and the wick materials. Finally, heat pipes can operate in any orientation, with or without gravity. This opens a wide panel of applications, in particular for space power systems.

On the downside, heat pipes advantages don't scale well with the reactor total thermal power. For bigger reactors, such as commercial ones, the weight and size of a heat pipe cooling system would be way too high. The lifetime of heat pipes is also limited, and it is not adapted for operations longer than 20 years. Test databases are really rare for heat pipes other than copper/water heat pipes (widely used in electronics) and long-term properties of high temperature heat pipes still has to be investigated. At last, the materials and working fluids used usually have a high thermal neutron absorption crosssection, which explains why it was never used for thermal reactors. 


\section{Variables}

$q_{t o t}:$ Total power input $(W)$

$T_{\text {sink }}:$ Entry sink temperature $(K)$

$C_{\text {air }}$ : Air heat capacity $\left(J \cdot \mathrm{kg}^{-1} \cdot \mathrm{K}^{-1}\right)$

$\dot{m}_{\text {air }}$ : Air mass flow in the sink $\left(\mathrm{kg} . \mathrm{s}^{-1}\right)$

$\theta:$ Tilt angle, taken from the horizontal to the pipe in trigonometric direction (Degrees)

$l_{e}:$ Evaporator section length $(m)$

$l_{a}$ : Adiabatic section length $(m)$

$l_{c}:$ Condenser section length $(m)$

$l_{\text {tot }}:$ Total pipe length $(m)$

$r_{i}$ : Inside pipe radius $(m)$

$r_{o}$ : Outside pipe radius $(m)$

$r_{v}:$ Radius of vapor space $(m)$

$d_{v}:$ Diameter of vapor space $(m)$

$A_{v}$ : Cross-sectional area of vapor flow passage $\left(\mathrm{m}^{2}\right)$

$A_{l}$ : Cross sectional area of liquid flow passage $\left(\mathrm{m}^{2}\right)$

$a:$ Characteristic dimension of the wick $(m)$

$z$ : Characteristic dimension of the mesh forming the wick $(m)$

$r_{\text {eff }}$ : Effective pore radius of the wick $(m)$

poro $_{\text {rad }}$ : Radial porosity of the wick (perpendicular to flow)

poro $_{a x}:$ Axial porosity of the wick (parallel to flow)

$\lambda_{\text {pipe }}$ : Pipe wall thermal conductivity $\left(W . K^{-1} \cdot m^{-1}\right)$

$\lambda_{\text {wick }}$ : Wick thermal conductivity $\left(W \cdot K^{-1} \cdot m^{-1}\right)$

$K$ : Wick permeability $\left(\mathrm{m}^{2}\right)$

$h_{\text {conv }}$ : Pipe to sink heat transfer coefficient $\left(W \cdot K^{-1} \cdot m^{-2}\right)$

$R_{\text {conv }}$ : Pipe to sink heat transfer resistance $\left(K \cdot \mathrm{m}^{2} \cdot W^{-1}\right)$

$\rho_{v}:$ Vapor density $\left(\mathrm{kg} \cdot \mathrm{m}^{-3}\right)$

$\rho_{l}:$ Liquid density $\left(\mathrm{kg} \cdot \mathrm{m}^{-3}\right)$

$\mu_{v}:$ Vapor dynamic viscosity (Pa.s)

$\mu_{l}:$ Liquid dynamic viscosity (Pa.s)

$L_{l \rightarrow v}$ : Latent heat of vaporization $\left(J \cdot \mathrm{kg}^{-1}\right)$

$\sigma:$ Surface tension $\left(\mathrm{kg} \cdot \mathrm{s}^{-2}\right)$

$\gamma:$ Specific heat ratio

$M:$ Molecular weight $\left(\mathrm{kg}^{\mathrm{m}} \mathrm{mol}^{-1}\right)$

$\lambda_{\text {fluid }}$ : Fluid thermal conductivity $\left(W \cdot K^{-1} \cdot m^{-1}\right)$

$\dot{m}_{a}$ : Axial vapor mass flow $\left(\mathrm{kg} \cdot \mathrm{s}^{-1}\right)$

$\dot{m}_{r}$ : Radial vapor mass flow due to evaporation or condensation $\left(\mathrm{kg} \cdot \mathrm{s}^{-1}\right)$ 
$U:$ Vapor average axial velocity $\left(m \cdot s^{-1}\right)$

$R e$ : Reynolds number

$R e_{r}:$ Radial Reynolds number

$f$ : Fanning friction factor

$P_{\text {sat }}(T)$ : Saturation pressure at temperature T $(P a)$

$T_{\text {sat }}(P)$ : Saturation temperature at pressure $\mathrm{P}(K)$

$d p_{i}$ : Vapor inertial pressure drop between $x$ and $x+d x(P a)$

$d p_{v}$ : Vapor viscous pressure drop between $x$ and $x+d x(P a)$

$d p_{l}$ : Liquid viscous pressure drop between $x$ and $x+d x(P a)$

$d p_{z}$ : Hydrostatic pressure drop between $x$ and $x+d x(P a)$

$g$ : Gravity acceleration on earth $\left(m . s^{-2}\right)$

$R$ : Universal gas constant $\left(J \cdot K^{-1} \cdot m^{-1}\right)$

\section{Heat pipe geometry:}

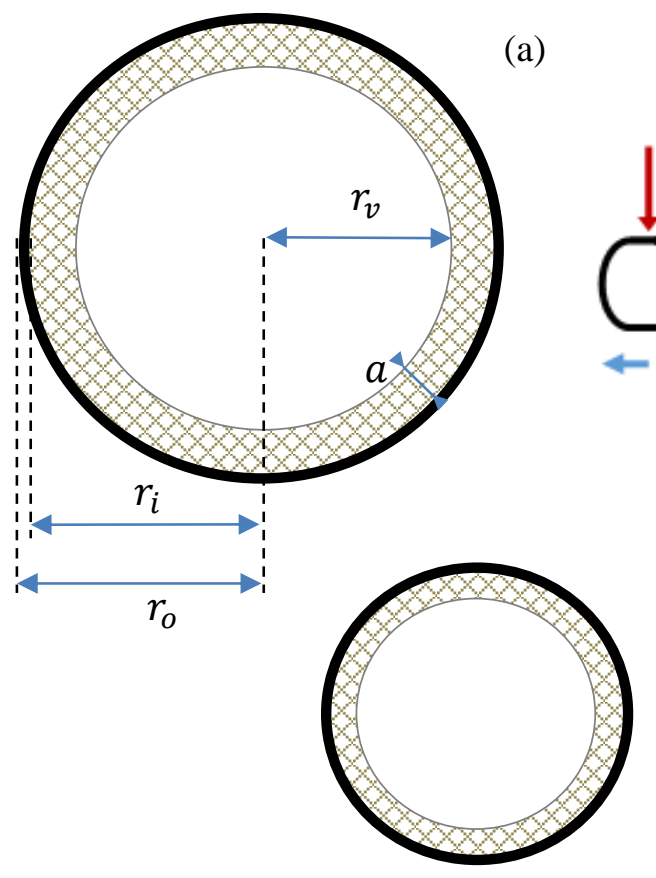

Homogenous wick

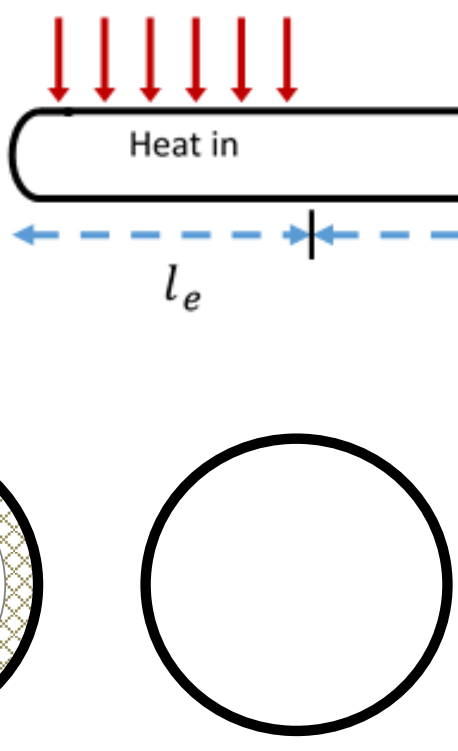

Wickless heat pipe

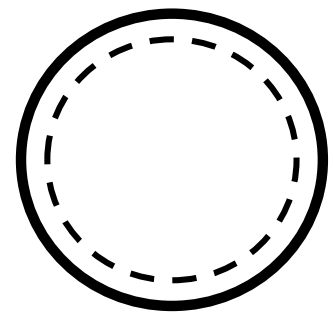

Annular gap (b)

(c)

Figure 30 | Cross-sectional views of heat pipes. (a) (b) Meanings of the main geometrical parameters. (c) Example of the most common heat pipe types. The annular gap geometry consists in a porous screen separating two open channels: one for the vapor and one for the liquid. 


\section{Model's equations}

\subsection{I.1. Code principle and main hypothesis}

The code has two main purposes: validating a heat pipe design compatible with our operating conditions and obtaining the temperature profiles inside the heat pipe during operations for stress simulations. It should also be able to simulate the effect of an adjacent heat pipe failure and take into account the position of the heat pipe in the reactor (i.e. variations of local power input but not total power). The model uses a discretized approach to calculate the working fluid temperature and pressure profiles, both in the vapor and liquid phase. The value obtained are then compared to the operating limits of the heat pipe to determine whether or not the input heat pipe geometry is compatible with the operating conditions.

The code uses a simple one-dimensional first order model and a lot of hypothesis to obtain an approximate, yet as faithful as possible, solution of the problem. First, we assume that all the liquid is contained inside of the wick, and all the vapor outside. The code thus uses single phase equations (except in some specific cases described later) to describe the liquid and vapor flows. Those flow are also supposed to be incompressible and laminar. In some cases, empirical correction factors are used to take into account turbulences and velocity profile changes along the axis perpendicular to the flow. The particular effects that could occur near the junctions between two sections or at the extremities of the pipe are neglected. Finally, transition effects are neglected and we assume the pipe has reached steady state and that the liquid and vapor phases are at saturation equilibrium.

Geometrically-wise, the pipe is supposed to be straight, of a constant radius and thickness, and divided into three sections: evaporator, adiabatic and condenser, in this order (there is no adiabatic sections before the evaporator or after the condenser). The heat transfer rate with the source will be an input as a function of the position. At the condenser section, we assume that the coolant is air at a constant mass flow, which enters the heat pipes array at the beginning of the condenser position and exits at the end of the array. The circulation of air is assumed ideal, so that the air temperature is constant perpendicularly to the pipes (even if the pipes temperature can vary at a given position for two different heat pipes). In particular, this implies that the sink temperature is independent of heat pipe failures or varying peaking factors and only depends of the total power and the average operating conditions.

\subsection{I.2. Incompressible one-dimensional vapor flow}

The vapor pressure at a given position is always calculated first, and the temperature is then determined by the Clapeyron relation between pressure and temperature at saturation.

If the vapor pressure $P_{v}(x)$ is known at a certain position $x$, the pressure $P_{v}(x+d x)$ can be found with:

$$
P_{v}(x+d x)=P_{v}-d p_{i}-d p_{v}
$$

The viscous vapor pressure drop can be calculated with the Hagen-Poiseuille equation in the case of a laminar flow or with the Fanning equation for a more general flow. The Fanning equation simply states that the pressure drop due to friction with the wick is equal to

Where $\tau$ is the shear stress at the wick.

$$
d p_{v}=\tau \cdot \frac{\text { contact area }}{\text { cross sectional area }}=\tau \cdot \frac{2 \pi r_{v} d x}{\pi r_{v}{ }^{2}}=\tau \cdot \frac{2 d x}{r_{v}}
$$

The Fanning friction factor is defined as the shear stress over the kinetic energy flow: 
Which yields:

$$
f=\frac{\tau}{\rho_{v} \cdot \frac{U^{2}}{2}}
$$

$$
d p_{v}=f \rho_{v} U^{2} \frac{d x}{r_{v}}
$$

Measurements of the friction factor have shown a dependency on the Reynolds number as follow:

$$
f=\left\{\begin{array}{l}
\frac{16}{R e} \text { if } R e<2000 \\
\frac{0.079}{R e^{0.25}} \text { if } R e>2000
\end{array} \text { where } R e=\frac{\rho_{v} U d_{v}}{\mu_{v}}\right.
$$

It can be noticed that if we replace $f$ by the first expression, we find the Hagen-Poiseuille equation. The second expression is an empirical correction used to take into account the turbulence effects. Another correction is used for compressible flows when the Mach number is greater than 0.2 (Chi, 1976). In this case, we use the following friction factor:

$$
f_{c}=f \cdot\left(1+\frac{\gamma-1}{2} M a c h^{2}\right)^{\frac{-3}{4}}
$$

Where Mach $=\frac{U}{C_{\text {sound }}}$ and $f$ is the friction factor previously calculated.

At this point, the average fluid velocity is still unknown, but it can be easily found with the power throughput $q_{a}(x)$ :

$$
\begin{aligned}
\dot{m}_{a}(x) & =\frac{q_{a}(x)}{L_{l \rightarrow v}}=\rho_{v} A_{v} U \\
U & =\frac{q_{a}(x)}{\rho_{v} A_{v} L_{l \rightarrow v}}
\end{aligned}
$$

The inertial vapor pressure drop is much harder to determine and varies depending on the model used and the authors. It first depends on the rate of mass injection or removal compared to the viscous effect, comparison that is described by the radial Reynolds number:

$$
R e_{r}=\frac{1}{2 \pi \mu_{v}} \frac{d \dot{m}_{a}}{d x}
$$

The most widely used first-order equation was developed by Cotter (1965) who assumed a uniform mass injection or removal rate on the length $d x$ and approximated the pressure gradient for a laminar incompressible flow in a cylindrical pipe to:

$$
\begin{gathered}
\frac{d p_{i}}{d x}= \begin{cases}\frac{6 \mu_{v} \dot{m}_{a} R e_{r}}{\pi \rho_{v} r_{v}{ }^{4}} & \text { if }\left|R e_{r}\right|<1 \\
\frac{S \dot{m}_{a}}{4 \rho_{v} R_{v}{ }^{4}} \frac{d \dot{m}_{a}}{d x} & \text { if }\left|R e_{r}\right|>1\end{cases} \\
\text { where } S= \begin{cases}1 & \text { in evaporator section } \\
\frac{4}{\pi^{2}} & \text { in condenser section }\end{cases}
\end{gathered}
$$

The difference of behavior between the evaporator and the condenser is due to a different radial distribution of the axial velocity when mass is injected (parabolic) or removed (cosinusoidal), effect that can be neglected when the mass change rate is small. This implies that for high heat transfer rate heat pipes, only $\frac{4}{\pi^{2}} \simeq 40 \%$ of the pressure lost by inertial effect is recovered in the condenser. The 
consequence is a lower pressure, and thus a lower temperature in the condenser, which reduces the heat exchanged with the sink.

\subsection{I.3. Liquid flow in capillary media}

As for the vapor, the liquid pressure is calculated by successive iteration using:

$$
P_{l}(x+d x)=P_{l}(x)+d p_{l}+d p_{z}
$$

Where the sign of the pressure drops varies depending on the conventions chosen. Here $x=0$ at the beginning of the condenser, meaning that $d p_{l}$ will be positive (as the liquid flows in the opposite direction as vapor) and $d p_{z}$ will be negative in case of gravity assist $(\theta>0)$. Note that in the case of the liquid, the inertial pressure drop due to mass removal or addition is negligible compared to the viscous drop.

The hydrostatic pressure gradient in a column of fluid gives:

$$
d p_{z}=-\rho_{l} g \sin \theta d x
$$

The viscous pressure drop depends on the nature of the wick. For homogenous wicks, the Darcy's law describing a fluid discharge rate through a porous media states:

$$
d p_{l}=\frac{\mu_{l} \dot{m}_{a}}{\rho_{l} A_{l} K} d x
$$

Where the permeability $K$ is a property of the wick, measured and given by the manufacturer.

When the liquid flows in an open channel, like circular arteries, grooves or an annular gap, the HagenPoiseuille equation for laminar flows applies. In the case of an annular channel, very common for liquid metal heat pipes, we can write the Hagen-Poiseuille locally, by assimilating the wall of the pipe and the mesh screen to two parallel plans separated by the distance a. The radial distribution of the axial fluid velocity is then:

Which gives an average velocity after integration:

$$
u(y)=y \frac{a-y}{2 \mu_{l}} \frac{d p_{l}}{d x}
$$

$$
U=\frac{a^{2}}{12 \mu_{l}} \frac{d p_{l}}{d x}
$$

Using $\dot{m}_{a}=\rho_{l} A_{l} U$ we find:

$$
\frac{d p_{l}}{d x}=\frac{12 \mu_{l} \dot{m}_{a}}{\rho_{l} a^{2} A_{l}} \simeq \frac{6 \mu_{l} \dot{m}_{a}}{\pi \rho_{l} r_{v} a^{3}} \quad \text { assuming } a \ll r_{v}
$$

For wickless heat pipes, we assume that $d p_{l}=0$.

\subsection{I.4. Power throughput calculation}

The power is known in the evaporator, being entered as an input for the calculations, and is constant in the adiabatic section. The remaining power in the condenser is calculated by successive iterations on the length of the section. Assuming the temperature of the fluid is known at the beginning of the condenser, the power exchanged with the sink along a subsection of length $d x$ is given by the newton law for forced convection:

$$
q_{r}=\frac{T_{\text {wall }}-T_{\text {sink }}}{R_{\text {conv }}} 2 \pi r_{i} d x
$$

Where $T_{\text {wall }}$ is the exterior temperature of the pipe. The temperature of air thus increases by:

$$
\Delta T_{\text {sink }}=\frac{q_{r}}{C_{\text {air }} \dot{m}_{\text {air }}}
$$


Given the fluid temperature and the power at $x$ in the condenser, the two previous equations give the power and sink temperature at $x+d x$. 


\subsection{I.5. Temperature drops}

The equations described above give the vapor temperature profile along the heat pipe, but not the radial profile of the temperature. Mechanical simulations need the temperature of the pipe wall and not of the vapor, it is thus important to calculate the radial temperature drops in the heat pipe. The code determines the thermal resistances of the different layers and interfaces (the vapor-liquid interface, the saturated wick and the pipe wall) following McLennan's works (1983). Across the vapor-liquid interface when evaporation or condensation occurs, the temperature drop can be found using the Clapeyron formula that gives after manipulation:

$$
Q \simeq \frac{P_{v a p} L_{l \rightarrow v}{ }^{2} A_{l v}}{\sqrt{2 \pi \frac{R T_{v a p}}{M}} \cdot R T_{v a p}{ }^{2}} \Delta T
$$

Where $A_{l v}$ is the area of the vapor-liquid interface and $Q$ is the heat transfer across the interface. The temperature drops across the wick and the wall are calculated assuming that the thermal resistance is only due to conduction. Using Fourier's law in cylindrical coordinates, the following expression can be found:

$$
Q \simeq \frac{2 \pi \lambda l}{\ln \left(\frac{r_{\max }}{r_{\min }}\right)} \Delta T
$$

Where $\lambda$ is the thermal conductivity of the layer, $l$ is the length of the pipe corresponding to the radial heat transfer $Q$ and $\frac{r_{\max }}{r_{\min }}$ is the ratio of the outer and inner radius of the layer. 


\section{Heat transfer limitations}

The temperature at one point is enough to determine the pressure and temperature profiles of the fluid (using Clapeyron's relations), but the total power obtained must still be compared to some operating limits of heat pipes. There commonly 5 operating limits, that all require a dedicated and precise study: viscous, sonic, entrainment, boiling and capillary limits. The viscous limit is relevant only for very low temperatures or high viscosity fluids, and it will be neglected further on.

\section{II.1. Sonic limit}

The sonic limit is the power that the vapor can transfer from the evaporator to the condenser when it has reached sonic speed in the pipe. As the vapor keeps accelerating until it reaches the condenser, this limit is calculated by assuming $M a c h=\frac{U}{c_{s o n}}=1$ at the end of the adiabatic section. Busse (1973) showed in this case that the heat transfer is equal to:

$$
Q_{\text {sonic }}=0.474 L_{l \rightarrow v} A_{v} \sqrt{P_{0} \rho_{0}}
$$

Where $P_{0}$ and $\rho_{0}$ are respectively the stagnation pressure and density of the vapor. An easier way to approximate the sonic limitation is by simply using:

$Q_{\text {sonic }}=\dot{m}_{a} L_{l \rightarrow v}=\rho_{v} A_{v} U L_{l \rightarrow v}$
As we assumed $U=C_{\text {sound }}=\sqrt{\frac{\gamma R T}{M}}$ at adiabatic section end, this yields:

$$
Q_{\text {sonic }}=\rho_{v} A_{v} L_{l \rightarrow v} \sqrt{\frac{\gamma R T}{M}}
$$

Where $T$ is taken at the end of the adiabatic section. This temperature can be hard to determine because it requires to know the power throughput. Although a good approximation can be found by assuming the temperature constant along the pipe, it may be inaccurate for low temperatures or long heat pipes. The code uses an iteration to determine the sonic limit. Given a certain evaporator exit temperature, a total power throughput is guessed, enabling the calculation of the vapor velocity at the evaporator exit and pressure drops along the adiabatic section. The viscous losses induce an increase of the vapor velocity that can be determined. At the end of the adiabatic section, if the Mach number is not equal to one, another power is guessed.

\section{II.2. Boiling limit}

If a certain liquid superheat is reached, nucleate boiling can occur and damage the pipe. If a bubble appears and grows in the liquid, the thermal conductivity will drop locally, leading to an overheating of the wall that could potentially damage it. For a bubble to appear, the temperature drop between the liquid on the wall surface and the fluid temperature far from the wall must enable the mechanical equilibrium of the bubble, given by the Laplace equation:

$$
P_{b}-P_{l}=\frac{2 \sigma}{r_{b}}
$$

Where $P_{b}$ and $r_{b}$ are respectively the pressure and radius of the bubble. The superheat needed to reach this pressure difference in a wick structure saturated with liquid can be found by integrating the Clausius-Clapeyron equation (Dunn and Reay, 1976):

$$
\Delta T_{\text {boil }}=\frac{2 \sigma T_{\infty}}{L_{l \rightarrow v} \rho_{v}}\left(\frac{1}{r_{b}}-\frac{1}{r_{e f f}}\right)
$$


Where $T_{\infty}$ is the temperature of the fluid far from the wall. We assume this temperature equal to the vapor phase temperature. The corresponding heat transfer rate can be found by using Fourier's law applied to a cylindrical pipe:

$$
Q_{b o i l}=2 \pi r l_{e} \lambda\|\vec{\nabla} T\|=2 \pi r l_{e} \lambda \frac{d T}{d r}
$$

Where $r$ is the radial coordinate of the cylindrical envelope for which the equation is written, $\lambda$ the thermal conductivity and $T$ the temperature in the liquid layer at the radial coordinate $r$. In the case of a wick saturated with liquid, we have:

By integrating between $r_{v}$ and $r_{i}$ :

$$
\lambda=\text { poro }_{\text {rad }} \lambda_{\text {wick }}+\left(1-\text { poro }_{\text {rad }}\right) \lambda_{\text {fluid }}
$$

Which yields:

$$
\int_{r_{v}}^{r_{i}} \frac{d r}{r}=\int_{T_{\text {vapor }}}^{T_{\text {wall }}} \frac{2 \pi l_{e} \lambda}{Q_{\text {boil }}} d T
$$

$$
Q_{b o i l}=\frac{2 \pi l_{e} \lambda \Delta T_{b o i l}}{\ln \left(\frac{r_{i}}{r_{v}}\right)}
$$

\section{II.3. Entrainment limit}

The Weber number compares the effects of the shear force imposed by the vapor on the liquid and the surface tension force due to the wick. We assume that when this number is greater than 1 , the entrainment of the liquid is too important (Droplets of liquid are entrained out of the wick and can create waves at the surface of the wick, blocking the vapor flow). This relation gives:

$$
W e=\frac{z \rho_{v} U^{2}}{2 \pi \sigma}=1
$$

Where $z$ is a characteristic dimension for entrainment. In the case of a wick or a mesh, $z$ is equal to the radius of the wire constituting it. Using $Q=\dot{m}_{a} L_{l \rightarrow v}=\rho_{v} A_{v} U L_{l \rightarrow v}$, we find the entrainment limit:

$$
Q_{\text {ent }}=A_{v} L_{l \rightarrow v} \sqrt{\frac{2 \pi \rho_{v} \sigma}{z}}
$$

Note that the entrainment is the strongest at the end of the adiabatic section, where the vapor as the highest velocity. The formula above should thus be calculated at the adiabatic section exit. For wickless pipes, the entrainment of liquid is the main limitation but it is much harder to determine. For a detailed model, the reader can refer to Faghri et al. works (1989).

\section{II.4. Capillary limit}

The wick structure in a heat pipe enables a pressure difference between the vapor and the liquid phase by capillarity. The maximum difference that can be achieved this way is:

$$
\Delta P_{\text {cap } \text { max }}=\frac{2 \sigma}{r_{e f f}}
$$


Where the effective pore radius $r_{\text {eff }}$ depends on the size of the wick pores and on the wetting angle of the liquid-wick contact. The capillary pressure built up along the pipe is the main pump for the liquid phase (figure 3), and it is also the main limitations of most wicked or screened heat pipes.

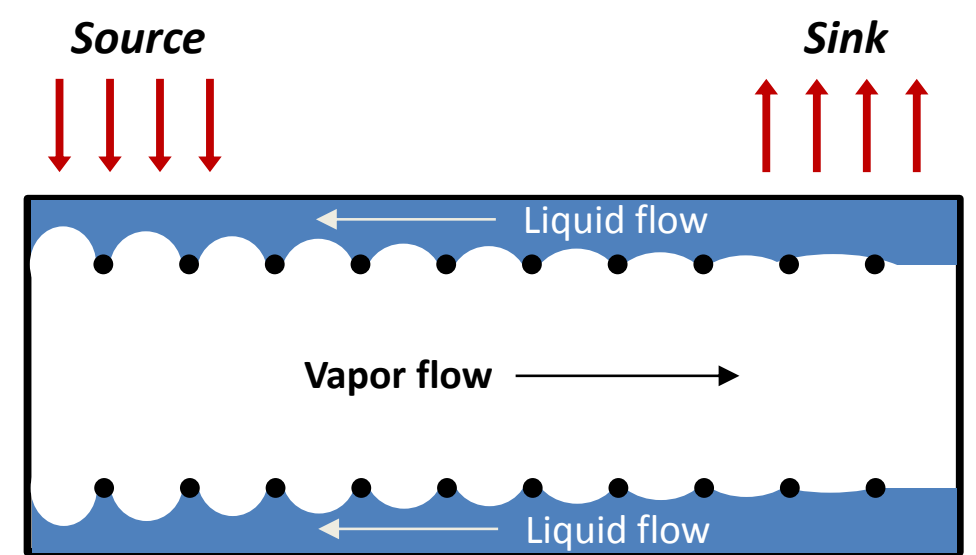

Figure 31 | Capillarity effects inside a heat pipe. Simplified view of the capillary pumping by the wick. The porous structure enables a pressure difference between the vapor and the liquid up to a certain point: the capillary limit.

If the maximum capillary pressure is lower than the pressure difference between the two phases, the wick will locally dry out and the heat pipe won't transfer enough heat. The heat pipe must then verify:

$$
\Delta P_{\text {cap } \text { max }} \geq \Delta P_{l}+\Delta \mathrm{P}_{v}+\Delta P_{i}+\Delta P_{z}
$$

Where $\Delta X=\int_{0}^{l_{\text {tot }}} d X$. It is important to be consistent with the sign conventions chosen, as the liquid and the vapor flow in different directions. In the later equations, the viscous total pressure drops $\Delta P_{l}$ and $\Delta P_{v}$ are taken positive, the inertial pressure drop $\Delta P_{i}$ is positive in the evaporator, negative in the condenser (the sum being always positive) and the hydrostatic pressure drop $\Delta P_{z}$ is negative in case of a gravity assist (tilt angle between 0 and 90 degrees, i.e. condenser end higher than evaporator end). In certain cases (for example when tilt angle is positive), the two phases keep the same pressure on a certain length $l_{w}$. The first point where the pressure are equals is called the wet point, above that point we assume that the two pressures are always equal. Thus, in order to calculate the capillary limit, the total pressure drops must be calculated from 0 to $l_{t o t}-l_{w}$ and not to the end of the condenser. 


\section{Results}

\section{III.1. Code validation}

One of the main challenge of a heat pipe simulation code is to find relevant experimental data to validate it. Liquid metal heat pipes have only been manufactured and tested by a few different entities such as the LANL, the ANL (Argonne National Laboratory) or the NASA (National Aeronautics and Space Administration). The operating limits are particularly difficult to measure, as it implies the failure of the pipe. Heat pipes being expensive systems, only the operating points are usually tested, and the limits are predicted by the codes. In order to validate the model used, some experiments will have to be carried out but it is already possible to compare the results of the simulation with other existing codes. When accurate geometrical data were available in the literature, simulations were run and compared with the ones made by the authors. Figure 4 is an example of comparison made with the predictions of the NASA on the performance of sodium heat pipes with annular gap.

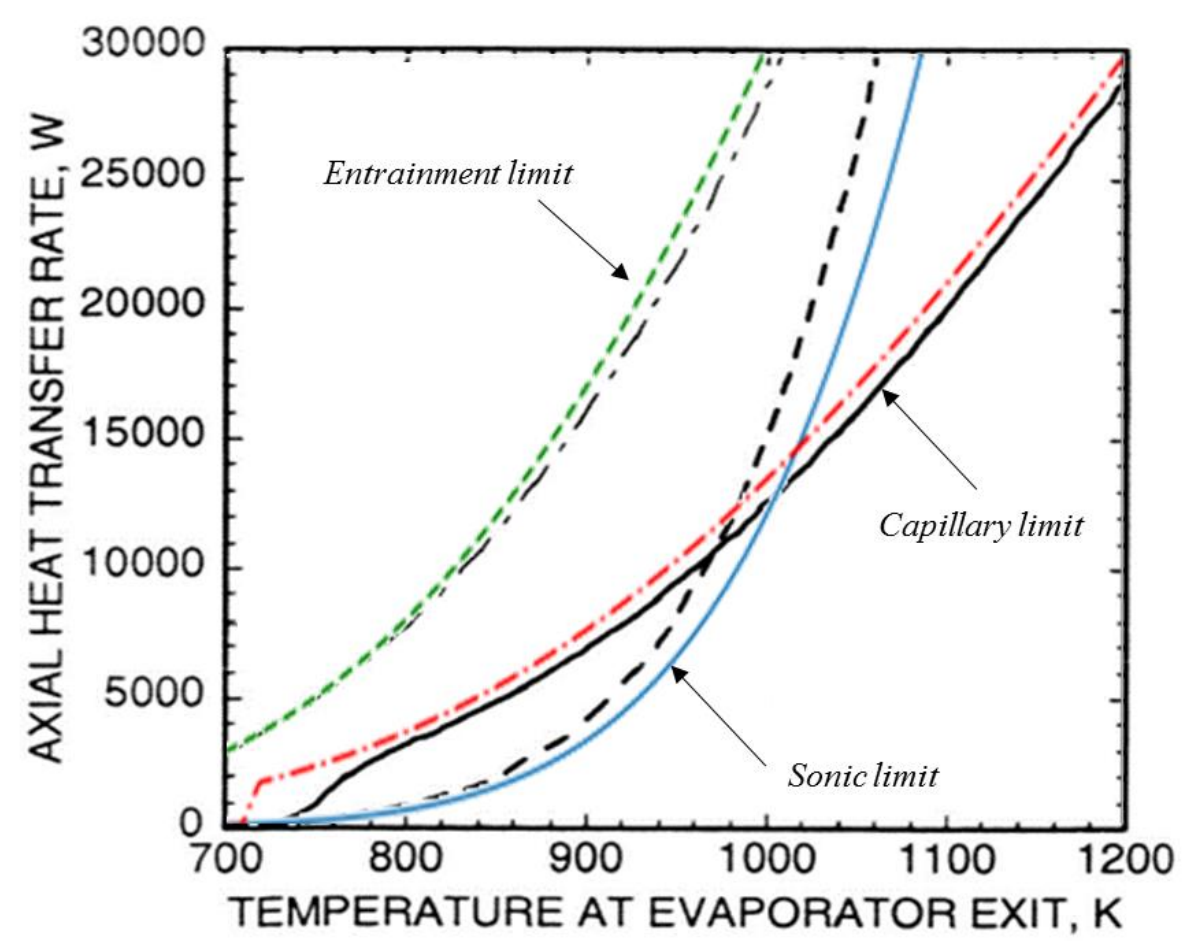

Figure 32 Comparison with NASA predictions for the SAFE-100a project. Black curves are NASA's predictions and colored ones are INL's predictions. The heat pipes used were $1.1 \mathrm{~m}$ long sodium pipes with a screened annular gap and an inner diameter of $1.5 \mathrm{~cm}$.

This method was repeated for a panel of existing liquid metal heat pipes and enabled us to compare our results with four other codes: the LANL, ANL, UNM (University of New Mexico) and NASA codes. Those comparisons have shown really similar results in every cases for both the capillary and sonic limits, that are the most likely to be reached for traditional wicked heat pipes. The entrainment and boiling limits calculations were significantly less consistent from a code to another. 
The mechanisms ruling those limits are still unknown and many different theories can be found in the literature. However, it is assumed by most authors that capillary limit will systematically be reached first in wicked pipes. Similarly, the boiling limit is not likely to be reached at normal operating temperatures. It becomes significant only for high temperatures, which the materials used already can't sustain.

Although this method can't be used to validate the model, it is a reassuring regarding our code. Knowing that the code's predictions are realistic, we can now design the experiments that will validate it more rigorously. These experiments will serve two different purposes: confirm that heat pipes fill our requirements and test the power limits for some given temperatures to compare with the code's predictions and ensure we have a decent power margin during operations.

\section{III.2. INL Heat pipe designing process}

Several design were investigated using the model previously described. These designs had to fill the following requirements, imposed by the geometry of the core and by the optimization of the power conversion efficiency:

- Exchange up to $8 \mathrm{~kW}$ from the source to the sink with a significant safety margin

- Operate between 900K and 1000K

- $\quad$ Length of $4 \mathrm{~m}$, with an evaporator section of $1.5 \mathrm{~m}$

- Outer diameter of $1.775 \mathrm{~cm}$

- Horizontal inclination

In this temperature range, the most interesting working fluids are either potassium or sodium. Sodium performs better at higher temperature, which was incompatible with the maximum fuel temperature allowed in the core. Since potassium performs slightly better than sodium in the 950$1000 \mathrm{~K}$ temperature range, it was chosen as the working fluid.

The first heat pipes investigated were "off-the-shelf" pipes built by private manufacturers. Generally extrapolated from the more usual water-copper heat pipes, those pipes were either built with a homogenous wick or no wick at all and, in both case, the requirements seemed impossible to reach. Wickless pipes, also called thermosyphons, only use gravity to return the liquid to the evaporator. Thus, it can't be operated horizontally and its operating limit is not capillarity but entrainment. Indeed, without the wick structure separating the vapor and the liquid, droplets of liquid are entrained much easier by the vapor flow, leading to the dry-out of the evaporator section or the flooding of the pipe if a wave is formed. For small radius heat pipes, the entrainment limit is too low for the thermosyphons to be a reliable option, as shown in Figure $5^{9}$.

\footnotetext{
${ }^{9}$ All the heat pipe limitations curves show the power limit as function of the vapor temperature at the evaporator exit
} 


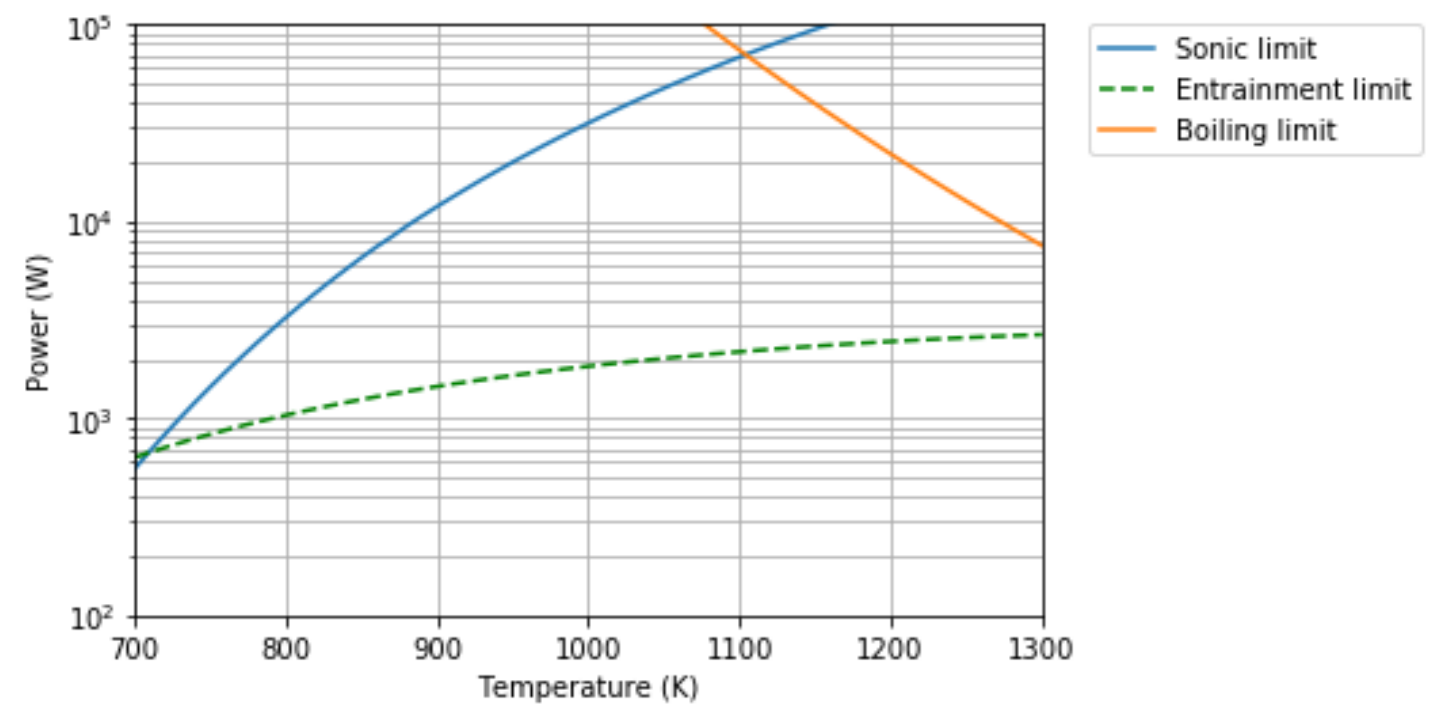

Figure 33 Power limitations without wick. 4-meter-long potassium heat pipe, $1.575 \mathrm{~cm}$ ID (Inner Diameter). The capillary limit is not represented because of the absence of a porous structure: the liquid return is only due to gravity and is more subject to entrainment by the vapor flow.

The other common design uses homogenous wicks to ensure the return of the liquid via capillary pumping. The wick is in direct contact with the wall of the pipe, meaning that all the liquid must flow back through the porous media. In this case, two main parameters must be taken into account to optimize the capillary limit: the pore radius and the permeability of the wick. The first one controls the maximum pressure difference sustainable between the vapor and the liquid phase. The second controls how easy it will be for the liquid to flow through the wick. To increase the capillary limit, the pore radius $r_{e f f}$ should be as small as possible, since the maximum pressure difference is $\Delta P_{c a p, \max }=$

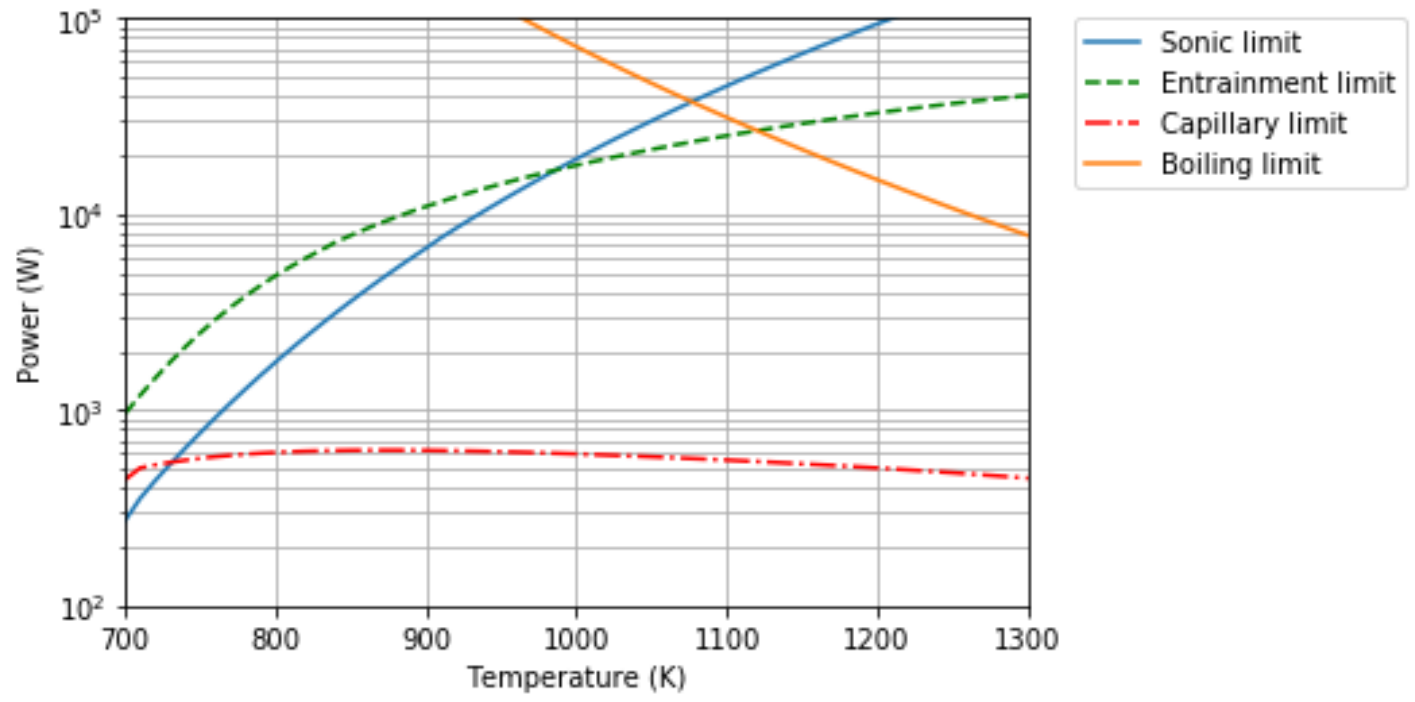

Figure 34 | Typical power limitations using a homogenous wick. 4-meter-long potassium heat pipe, $1.575 \mathrm{~cm}$ ID. The wick prevents entrainment but increases drastically the viscous loss of the liquid, leading to a very low capillary limit. 
$\frac{2 \sigma}{r_{e f f}}$, and the permeability as large as possible. However, it is not possible to optimize those two parameters at the same time because smaller pore radius will induce a lower permeability and thus higher viscous loss in the liquid phase. This flaw, inherent to homogenous wicks, prevents its use in the case of long thin heat pipes because of the systematically too low capillary limit. Figure 6 shows the performance obtained with a homogenous wick with our geometrical constraints.

In order to increase the capillary limit, the viscous loss of the liquid flow must be significantly reduced. This can be achieved by letting an open channel for the liquid to come back to the evaporator. This channel must be covered by a wick or a screen to prevent entrainment by the vapor and enable a pressure difference between the two phases by capillarity. The most commonly used geometry in the literature is the annular gap. A mesh screen is placed in the tube and separated from the wall by spacers, letting an annular gap between the screen and the wall in which the liquid can flow freely. The pore radius of the screen can thus be chosen as small as possible, without affecting the liquid axial flow (as long as the radial permeability is high enough to enable the evaporation and condensation processes). Regarding our requirements, this solution outperforms by far the thermosyphons and homogenous wick pipes. Three parameters intervene in the optimization of the capillary limit with this geometry: The size of the gap, the thickness of the screen and the pore radius. Contrary to homogenous wicks, these three parameters can be optimized simultaneously, explaining the higher performances. The pore radius should be as small as possible to increase the maximum pressure difference between the two phases. The screen should also be taken as thin as possible since it increases both the vapor and the liquid flow area. However, the annular gap has an optimal value between 0 and $r_{v}$ because it affects the two flow areas in opposite ways. When testing a mesh, this optimal value should always be determined first as it will give the best performances. Many mesh were simulated and figure 7 shows the results found with the best mesh found in the literature, with an optimized gap size (see table 1).

Pipe geometry

\begin{tabular}{|l|r|}
\hline Evaporator length $(\mathrm{m})$ & 1.5 \\
Adiabatic length $(\mathrm{m})$ & 0.3 \\
Condenser length $(\mathrm{m})$ & 2.1 \\
Outside pipe radius $(\mathrm{mm})$ & 8.875 \\
Inside pipe radius $(\mathrm{mm})$ & 7.875 \\
\hline Wick geometry & 0.7 \\
\hline Annulus thickness $(\mathrm{mm})$ & 1 \\
Screen thickness $(\mathrm{mm})$ & 0.02 \\
Half of wire diameter $(\mathrm{mm})$ & 0.015 \\
Effective pore radius $(\mathrm{mm})$ & 0.706 \\
\hline
\end{tabular}

Table 15 | Geometrical parameters of the current INL heat pipe design. The mesh used for the screen is a stainless steel 400-mesh that has already been manufactured at the Wright-Patterson Research \& Development Center. 

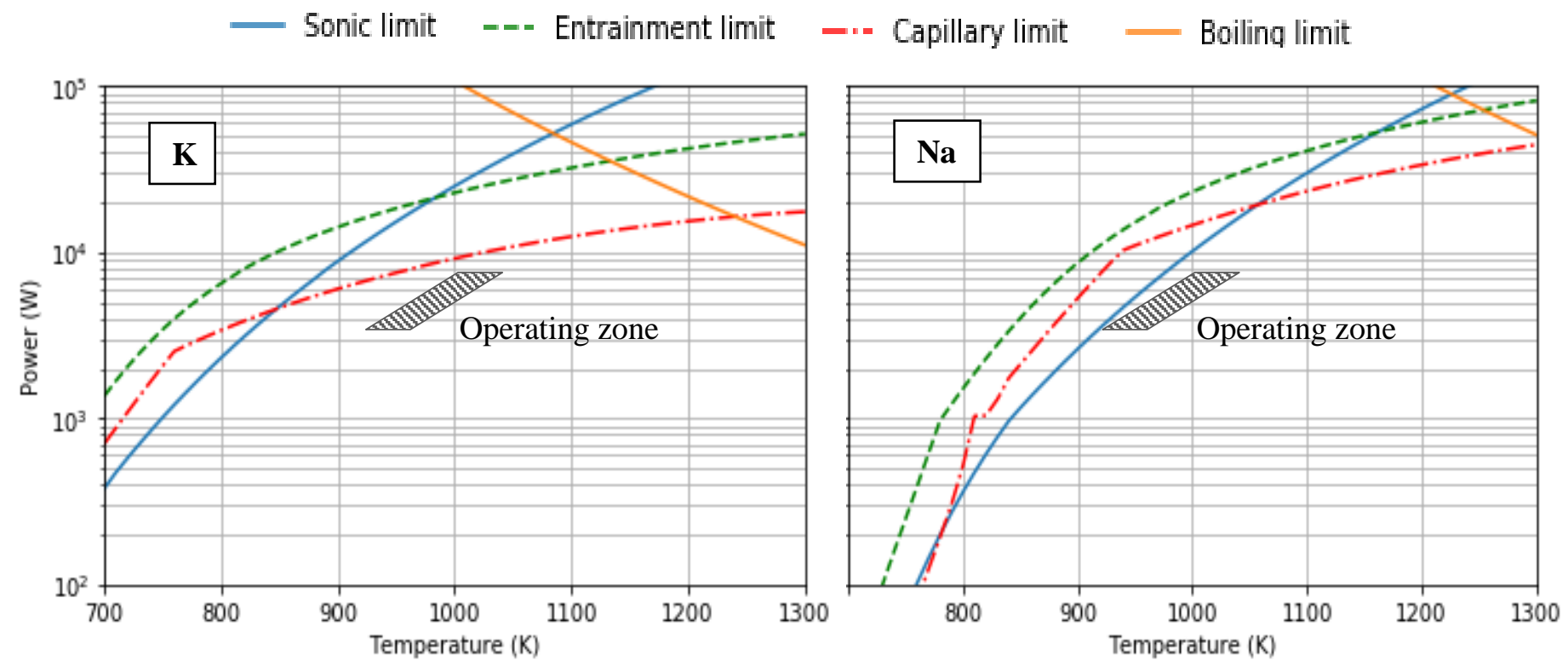

Figure 35 | INL heat pipe design limitations using potassium (left) or sodium (right). In both cases, the heat pipe maximum power in the $900-1000 \mathrm{~K}$ temperature range is very close to the operating power range $(3.5-7.5 \mathrm{~kW})$. Due to its high sonic limit, potassium is a more relevant choice for this design.

As expected, the boiling and entrainment limit are high enough and should not require any specific development efforts. In the case of potassium, the sonic limit is also particularly high at temperatures above $900 \mathrm{~K}$. The sonic limit is the main reason why potassium outperforms sodium in this temperature range. Since sodium has a higher evaporation temperature, the vapor pressure is significantly lower than potassium vapor pressure below $1000 \mathrm{~K}$ and thus the sonic speed is reached at lower power throughputs. Although the capillary limit is higher for sodium, the sonic limit doesn't let enough margin, and it can only be increased by enlarging the pipes. The capillary limit on the contrary can be improved by several ways. This is why potassium was chosen as the working fluid (Sodium would be the obvious choice if the operating temperature were to be increased).

The sonic limitation may not be an issue with potassium but the capillary limit is relatively close to the $8 \mathrm{~kW}$ target at $1000 \mathrm{~K}$, and the margin is still too low to be confident with this design. Several options are available to increase this margin. The first option is to change the geometry of the pipe, by using another method to return the liquid to the evaporator. Other wick structures in particular have been developed for this purpose, like composite wicks or circular arteries wicks. On this regard, Los Alamos National Laboratory is currently developing much more performant wicks. Those wick designs must still be patented and thus no additional details were available. The second option is to incline the pipe to help the liquid flow with gravity. As shown on figure 8 , a satisfying margin can be obtained when using vertical heat pipes, but it also adds complexity to the engineering design. Finally, a third option would be to increase the radius of the heat pipes (figure 9). This would imply higher losses of neutron in the core due to absorption by the pipes, leading to a decrease of reactivity. To counterbalance this effect, the core needs more fuel, adding extra costs to the project. 


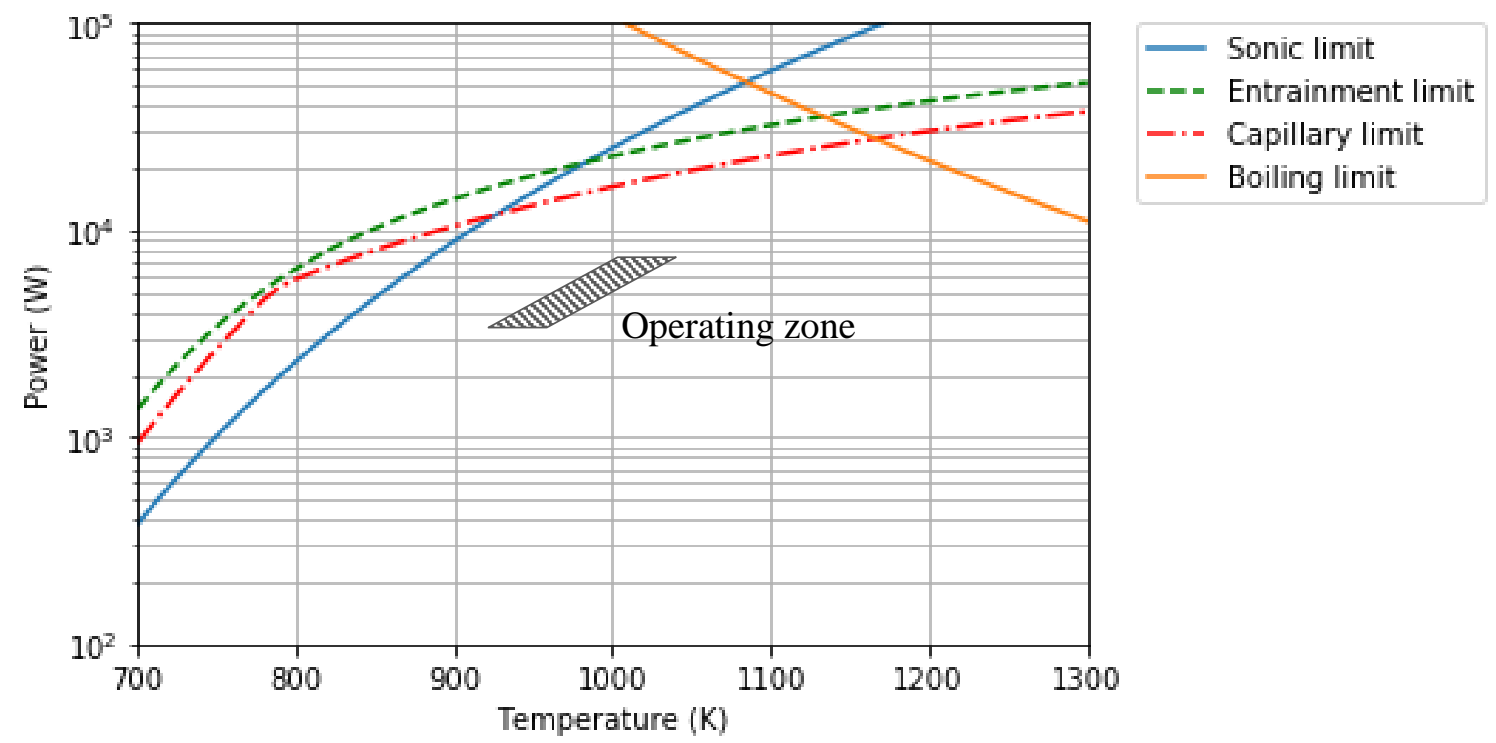

Figure 36 | Vertical heat pipe limitations. Table 1 was used for the geometrical parameters. Gravity assist for the liquid return to the evaporator increases the capillary limit and a significant margin is obtained.

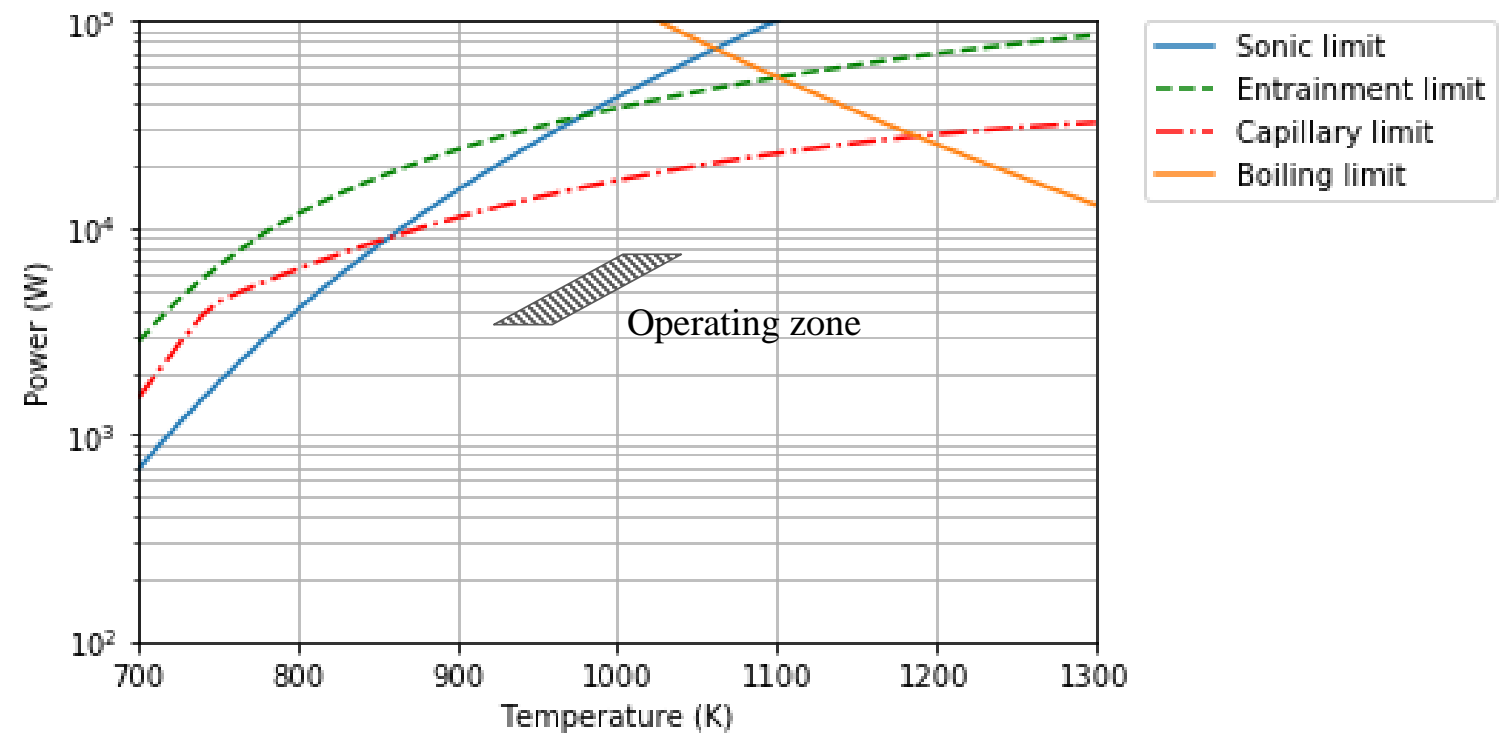

Figure 37| Horizontal, larger heat pipe limitations (+4 mm ID). Other parameters were taken following table 1. A significant margin is also obtained, at the cost of an addition of fuel in the core in order to keep the same reactivity

At this point of the project, the three options are being investigated. In particular, neutronic studies were conducted to determine the number of additional fuel rods needed if the radius of the heat pipes is increased. However, the most reliable option seems to be the modification of the wick. Figure 10 gives the LANL predictions for the new wick currently being designed. Although the details of this 
wick remain unknown, it can be seen that the capillary limit was drastically increased, at the cost of a lower sonic limit. This design would give a comfortable margin at $1000 \mathrm{~K}$ that would most likely compensate the modelling errors and the manufacture flaws.

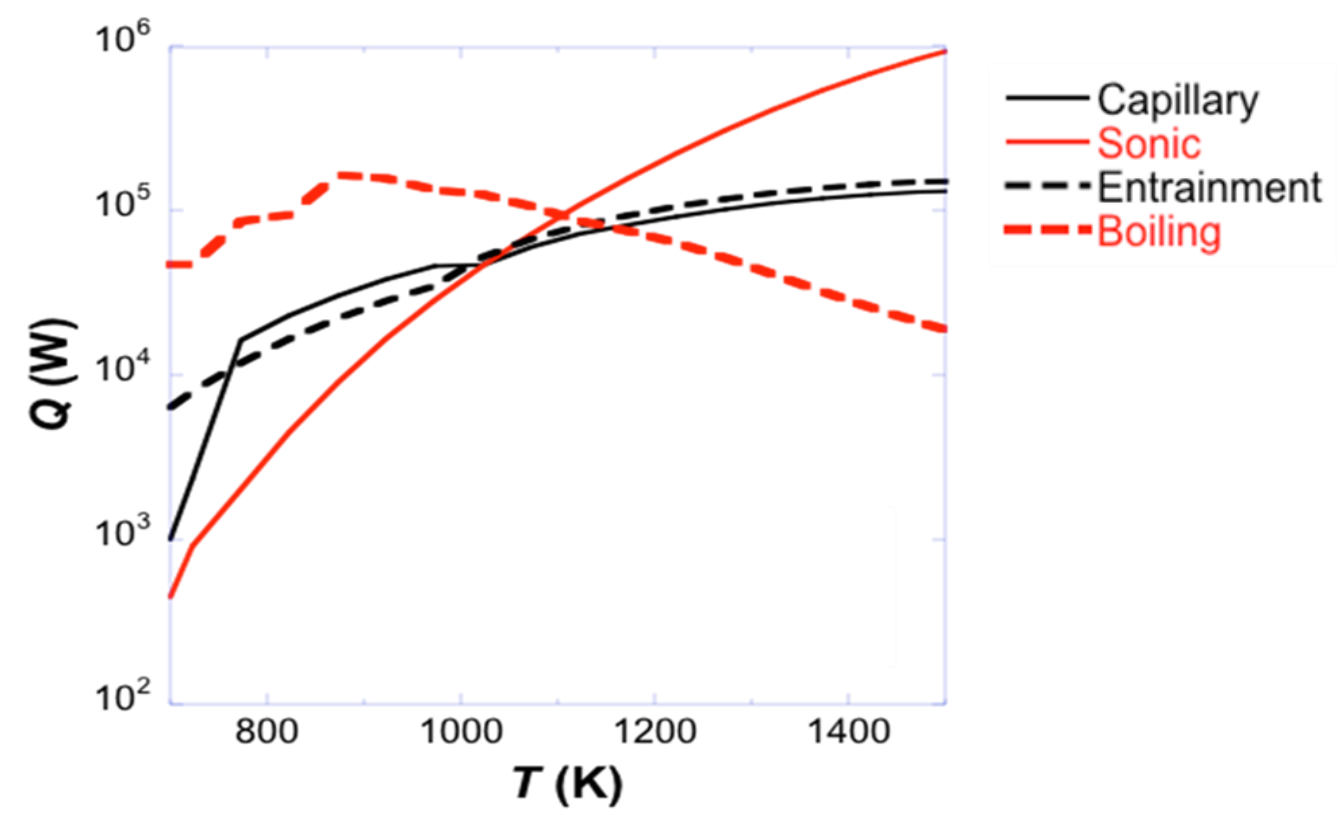

Figure 38 | LANL heat pipe design limitations. Horizontal heat pipes with the same dimensions as in table 1 . These predictions were calculated by LANL using a new type of wick currently in development and present the highest margin of all three options.

We are now confident in the ability of heat pipes to efficiently and safely withdraw heat from the reactor core. The three options to improve the design give satisfying results and the choice will be ultimately driven by their cost-effectiveness.

\section{III.3. Heat pipe thermal analysis}

The most decisive part of the heat pipe designing process is to find a geometry that gives the targeted power limits. For given lengths of evaporator, adiabatic and condenser sections, the pressure and temperature profiles are almost independent of the wick design. Although the viscous losses play a major role at the scale of the capillary maximum pressure difference, it is not as significant compared to the actual pressure of the vapor. This explains that the vapor phase is nearly isothermal along the pipe. Its temperature mostly depends on the heat exchanger at the sink and on the thermal resistances of the wall and the wick. The heat exchange with the sink can be either convective, radiative or a coupling of the two. Here, it is assumed that the transfer is purely convective. The coolant properties, mass flow, temperature and conductive transfer coefficient are given as input. The code is then able to determine the vapor pressure and temperature profiles in the pipe as well as the pipe's wall and wick temperature. The elevation of sink temperature is also calculated. For this reactor design, the coolant is air, pre-heated and injected in the heat pipe array at the condenser beginning level. The air removes heat by forced convection. An in-depth study of the power cycle was conducted to determine the best values for the heat exchanger properties. Those values can be found on Table 2. 


\section{Sink heat exchanger}

\begin{tabular}{|l|r|}
\hline Condenser length (m) & 2.1 \\
Coolant & Air \\
Inlet temperature (K) & 725 \\
Mass flow (g.s-1) & 20.12 \\
Heat transfer coefficient (W.K-1.m-2) & 326 \\
\hline
\end{tabular}

Table 16 | Parameters of the sink heat exchanger design.

Figure 11 shows the results obtained with the design previously detailed for a power throughput of $4.5 \mathrm{~kW}$. The vapor temperature only varies by $4 \mathrm{~K}$ from one end to the other, which is consistent with the nearly isothermal hypothesis. This temperature is controlled by the sink properties. It is generally chosen as high as possible to increase efficiency and the heat transfer capacities of the pipe, but below the point where the fuel temperature would reach its limit.
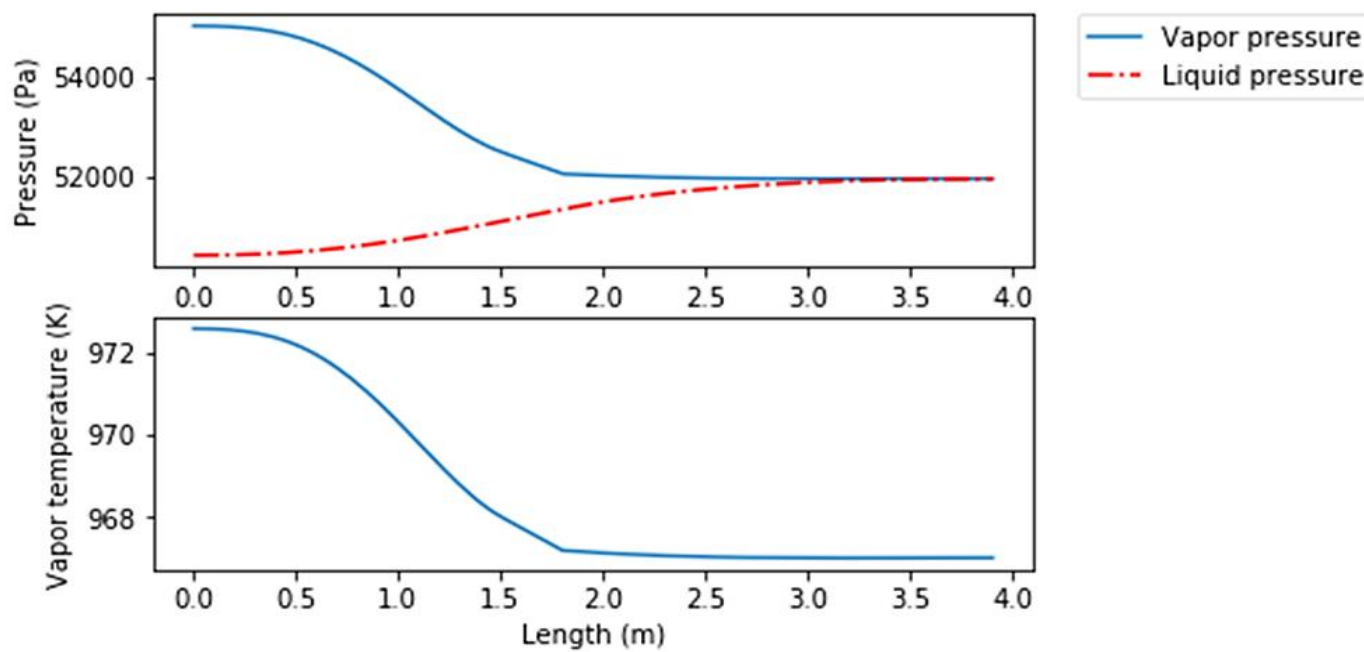

-.- Liquid pressure

Figure 39| Pressure and temperature profiles of the working fluid. The length axis starts at the evaporator beginning. These predictions were calculated based on tables 1 and 2 designs, for a total power of $4.5 \mathrm{~kW}$ and a parabolic heat input profile. Air temperature at condenser exit is $926 \mathrm{~K}$

Many other assessments made through this report can be observed on the graphs. The vapor pressure is relatively low, even at $970 \mathrm{~K}$, with a pressure of approximately half an atmosphere. Regarding safety issues, it means that leaks will be less problematic, compared for example with pressurized water in the commercial PWR. Potassium and sodium are very reactive though, especially with oxygen, creating other safety issues to deal with. Besides, this pressure is still one order of magnitude greater than the total pressure drop in the pipe, leading to an almost constant temperature. This pressure drop is mostly due to vapor, the liquid pressure drop remains low compare to an homogenous wick thanks to the open channel created by the screen. The evaporator section sees the 
most losses due to the addition of viscous and inertial effects. The vapor pressure drops in the condenser section are way smaller because the pressure recovery due to condensation counterbalances the viscous effects.

Vapor temperature is relevant to understand the mechanisms of heat pipes and calculate the power limits, but the exterior wall temperature is a more relevant output since it is used for stress simulations and heat transfer calculations.

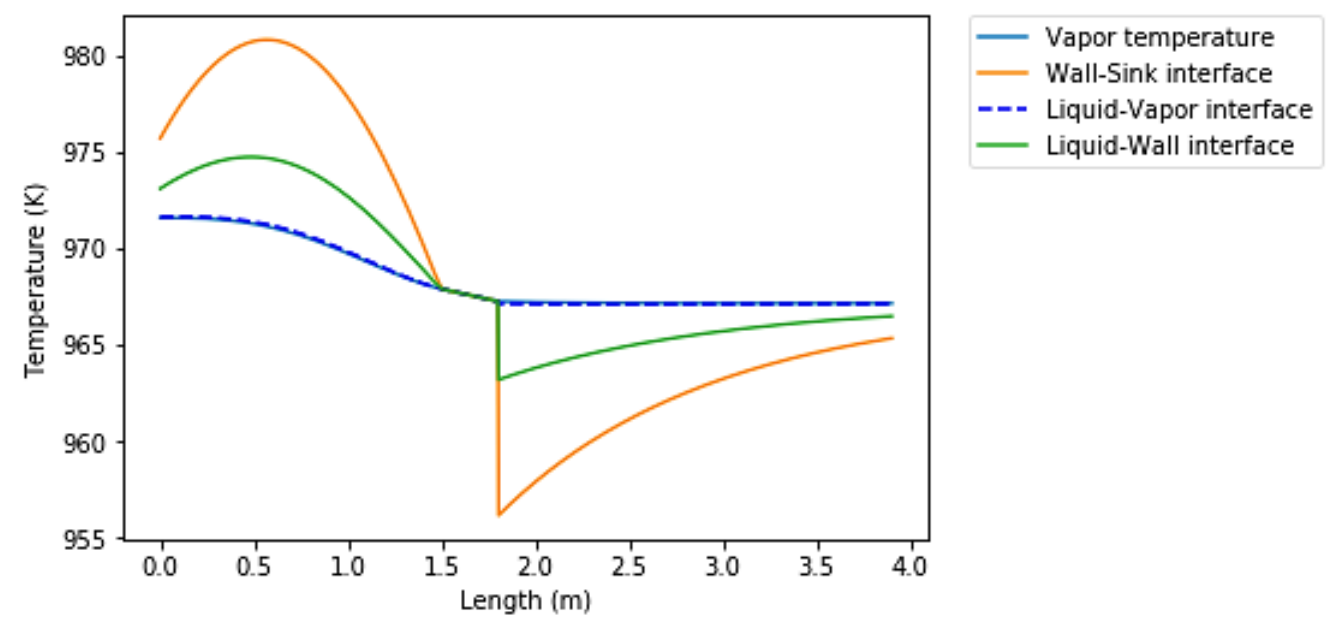

Figure 40 | Temperature profiles of radial interfaces. Simulations following tables 1 and 2, for a total power of $4.5 \mathrm{~kW}$ with a parabolic heat input profile at the evaporator. Convection in the liquid phase and axial conduction are neglected. This induces the discontinuities at the adiabaticcondenser interface, along with the perfect isolation hypothesis.

In Figure 12, it can be seen that the temperature drops of the wall are not negligible anymore $(\sim 25 \mathrm{~K})$ and it will have to be taken into account in the simulations. The radial difference between the liquid temperature at the liquid-vapor interface and at the liquid-wall interface is due to our hypothesis of neglecting convection in the liquid phase. In reality, the temperature drop in the liquid phase at equilibrium is probably smaller. We also ignored the axial conduction in the wall of the pipe because $\frac{r_{0}-r_{i}}{l_{\text {tot }}} \ll 1$. The discontinuity of the curve at the section junctions is one of the consequences. The adiabatic section is assumed to be perfectly isolated, without any radial heat loss, meaning that all the layers will have the same temperature. In the absence of axial conduction, this leads necessarily to an axial discontinuity of the wall temperature.

Several cases are investigated for each design to simulate different operating conditions, including accident conditions. Depending on its position on the core, a heat pipe can exchange various powers, going from $83 \%$ to $119 \%$ of the average power seen by each individual heat pipe (see figure 13). In case of a heat pipe failure, its direct neighbors must exchange approximately $17 \%$ more power since every heat pipe has six neighbors. Even if the individual power throughput of the heat pipes can vary, the total thermal power exchanged with the sink is the same (5 MWth). Assuming perfect circulation of the coolant in the heat exchanger, it means that every heat pipe sees the same sink temperature at a given position. Heat pipes will be hotter as its power throughput increases. Five cases were simulated:

- Minimum power input, no adjacent failure $(3.7 \mathrm{~kW})$

- Average power input, no adjacent failure $(4.5 \mathrm{~kW})$ 
- Maximum power input, no adjacent failure $(5.4 \mathrm{~kW})$

- Maximum power input, 1 adjacent failure $(6.3 \mathrm{~kW})$

- Maximum power input, 2 adjacent failures $(7.3 \mathrm{~kW})$

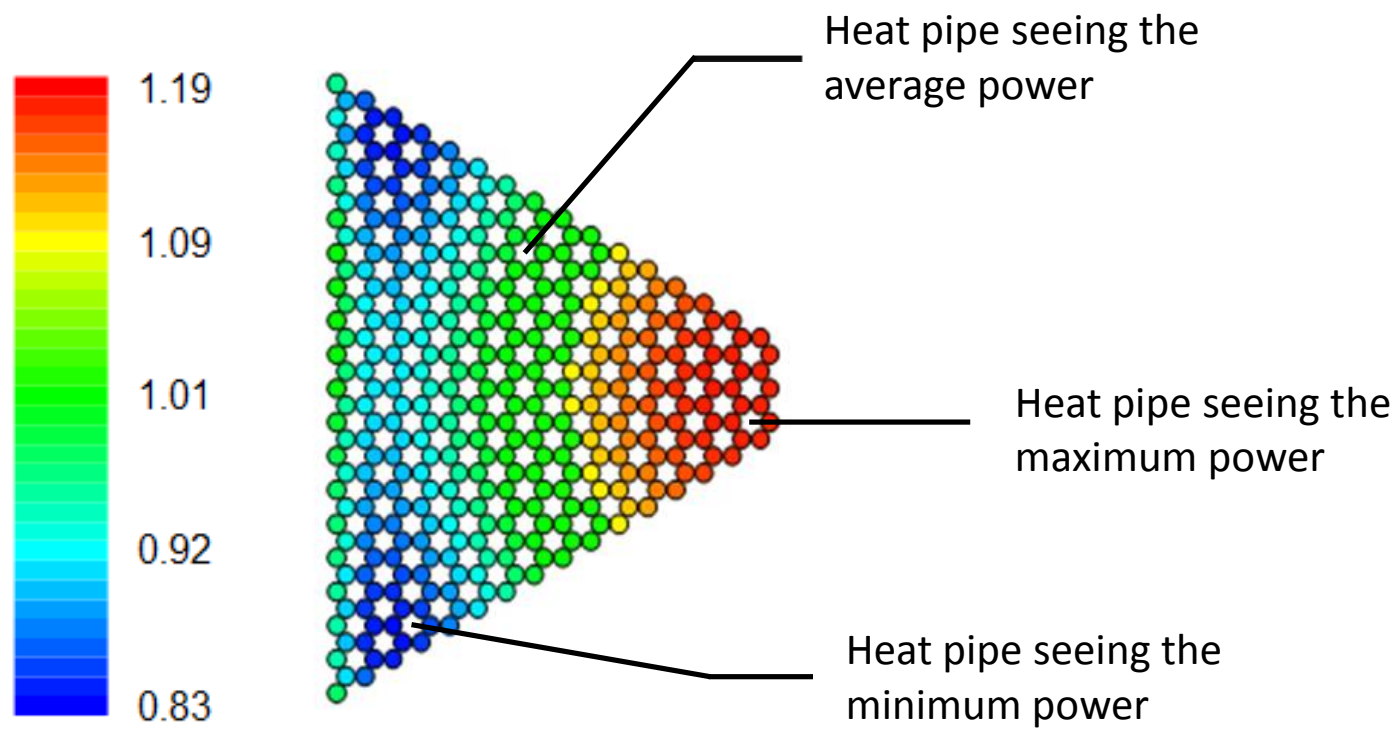

Figure 41 | Power peaking factor distribution in the core. (LANL 2015 design, DOE report LAUR-15-28840) Only one sixth of the core is represented. Numbers are averaged on the full length of the rods and local peaks may be higher. The pipe design chosen must be able to operate in every region of the core with up to two adjacent heat pipes failure.

The purpose of this study was to prove that even two adjacent failures in the highest peaking factor zone would not create a chain failure reaction. As mentioned above, the operating temperature of heat pipes increases with the total power input. The capillary limit, which is usually the limiting factor, also increases with temperature but with a lower slope, meaning that the power margin reduces as the power input increases. Figure 14 shows the different operating points for each case, using tables 1 and 2 parameters, where the inlet sink temperature has been decreased to limit the operating temperatures to $1000 \mathrm{~K}$. 


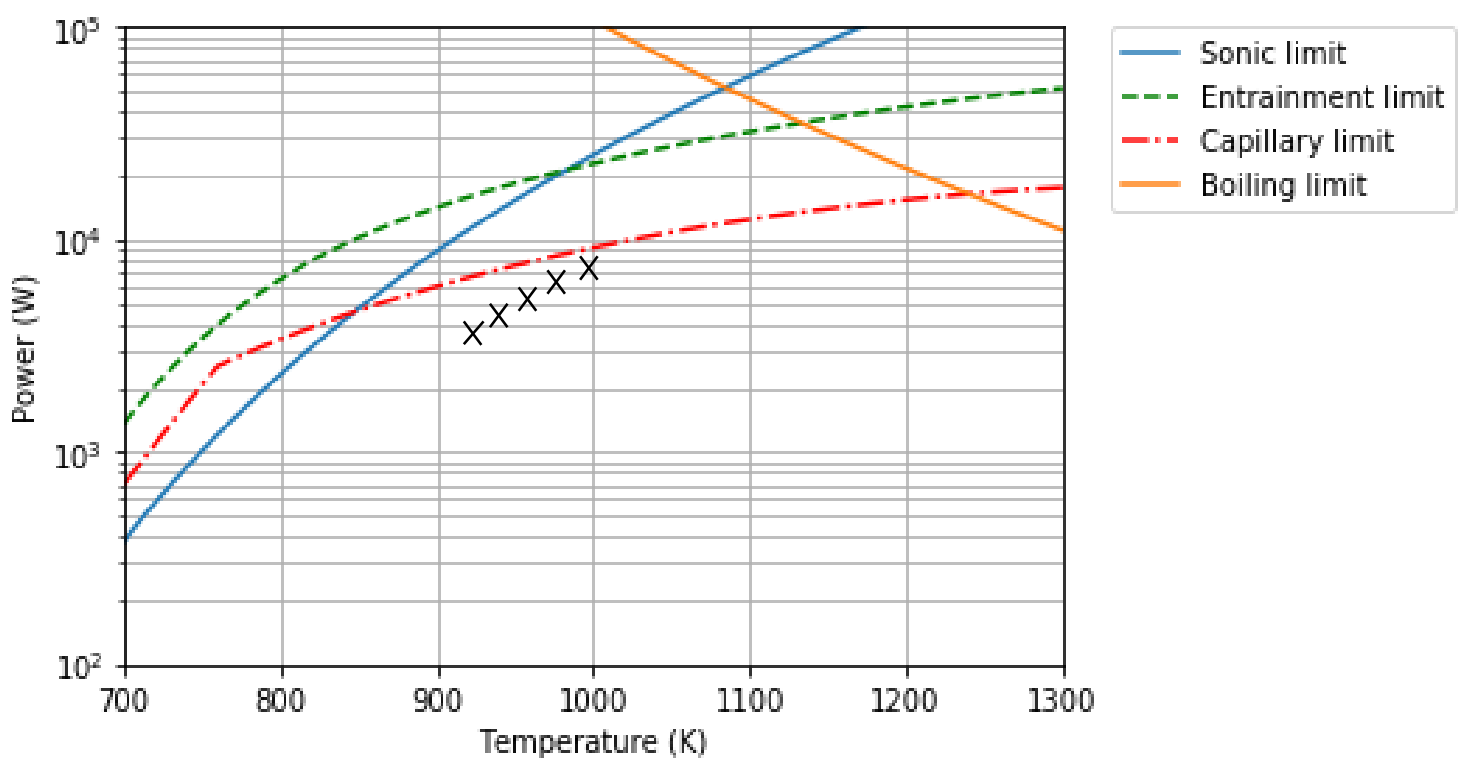

Figure $42 \mid$ INL heat pipe operating points for various power input. The two points of highest power correspond to accident scenarios where one or two adjacent heat pipes failed; the three others points are under normal operating conditions. The air mass flow and temperature at the sink were taken to have a temperature of $1000 \mathrm{~K}$ in the worst case scenario.

It is important to notice for the stress simulations that, at the same time, two heat pipes can have a temperature difference of up to $80 \mathrm{~K}$. Near the hottest heat pipes, fuel temperature can increase significantly, since it has to counterbalance both the temperature increase of the pipe and the higher power to transfer. Heat pipes can be designed to sustain extremely high temperatures but the tolerance for the fuel elements is much lower. Rigorous thermal analysis of the core will have to be made to determine the maximum fuel temperature as function of the heat pipe temperature. This will decide the properties of the sink, and thus the efficiency of the power cycle.

As mentioned earlier, the power margins are not high enough with this design to comfortably compensate the modelling errors and the manufactures flaws. In this particular case, the power margin varies from $90 \%$ under normal operating conditions to $20 \%$ for the maximum power input scenario. However, since the operating points will remain at the same power if the wick, the orientation or the pipe radius is modified, there will be a large margin once one of the option will have been chosen. 


\section{Conclusion}

A simple steady-state model of heat pipe thermodynamics was implemented in python to evaluate the performances of such systems for the cooling of a small modular fast reactor. The code uses strong hypothesis but show similar results as other codes implemented by LANL, NASA, ANL or University of New-Mexico. Specifically adapted to the INL project of small modular reactor, the code provides performance limitations and temperature profiles for the most common types of heat pipe. The simulations of existing wicks, both off-the-shelf and on the literature, led to a screened annular gap design able to transfer $9 \mathrm{~kW}$ at $1000 \mathrm{~K}$. Several options are being investigated to increase the power margin. Three options give greater margins than required, consolidating the choice of heat pipes as a safe and effective way to remove heat from the reactor core.

Many simulations and experiments still have to be carried out on heat pipes. A precise thermal analysis of the fuel elements is needed to determine the maximum heat pipe temperature allowed. The code will have to be validated by experimental data on real 4-meter potassium heat pipes and designing the experiments correctly will be a crucial point. The prototypes will have to be thoroughly tested in order to prove the reliability of heat pipes when exposed to radiations and high temperature liquid metal and air for several years. Finally, for a real accurate simulation of heat pipes performance, the code should include a transient state simulation, to evaluate its behavior during the startup of the reactor, when the working fluid is in the solid state. 


\section{Code main procedures}

Before going into the actual calculation code, we will mention the two side files that contain the classes and the graphical interface. The first one contains the definition of several classes used to regroup parameters that are often modified by categories. It also has some functions that run basic operations on those parameters, like recalculation for a different temperature. The second file is used to create the graphical user interface. It first places all the entries and labels on the interface and then defines some functions to look for an input file (both for geometry and for the heat input), to set a save path and to choose the calculation options.

\subsection{Option 1: Temperature and pressure profiles}

We saw that the knowledge of the temperature at a single point in enough to determine the temperature and pressure all along the heat pipe. The principle of the calculation loop is thus to guess a temperature at a certain point, deduce these profiles and check if the heat exchanged at the condenser is equal to the targeted power. If not, another temperature is guessed depending on the power found (lower temperature if the exchanged heat is greater than the target, higher otherwise). Once the powers match, the whole calculations is run again but by keeping the sink temperature profile of the previous calculations. This second run enables to take into account the variation of power between different heat pipes. The coolant going through all the heat pipes at once, it sees only an average power and will receive the same global heat independently of local variations. The heat pipes on the contrary can see a different power input from the source depending on the position in the core and the possible failures of other adjacent heat pipes. It is possible in the input interface to specify the peaking power factor at the heat pipe location and the number of adjacent failures to see the effect of such parameters on the heat pipe performance. Not that in the case of a single heat pipe simulation and not an array, the peaking factor would be 1 and the number of adjacent failure 0 . See annex 1 for a more detailed flowchart. At the end of the function, the temperature and pressure profiles of the heat pipe are displayed on graphs, and all the data is written in an output file.

\subsection{Option 2: Power limits}

The principle is simpler than option 1: after reading the input data, the pressure drops are calculated along the heat pipe for each temperature increment between the two bounds chosen by the user. Once determined, the limits are calculated and added to the plot. Once all the calculations are done, a graph with default axis is shown, and a new window lets the user change those axis and choose to use or not a logarithmic scale. As for option 1, all the data displayed on the graph is also written in an output file.

\subsection{User instructions}

To run the code, be sure to have the three files (main code, classes and interface) in the same folder. When launching the main file, a user interface appears to enter the input data. The input data can either be put manually or via an input file. An example of input file can be found in annex. Note that it is necessary to have all the parameters relevant for the geometry chosen in the input file, an error will appear if one is missing.

The power exchanged with the source can either be a number or a file. If it is a number, the code will consider this number to be the total power input and will assume the heat transfer is linear 
between the evaporator and the source. To allow non-linear heat transfers, it is possible to specify the heat transfer distribution via an input file. An example of such a file can be found in annex.

Some versions may have a "default values" button, which sets all the parameters to a certain set of pre-entered values. This function is mostly for developing purposes and should be remove for any freely available version. 


\section{Annex 1: Option 1 flowchart}

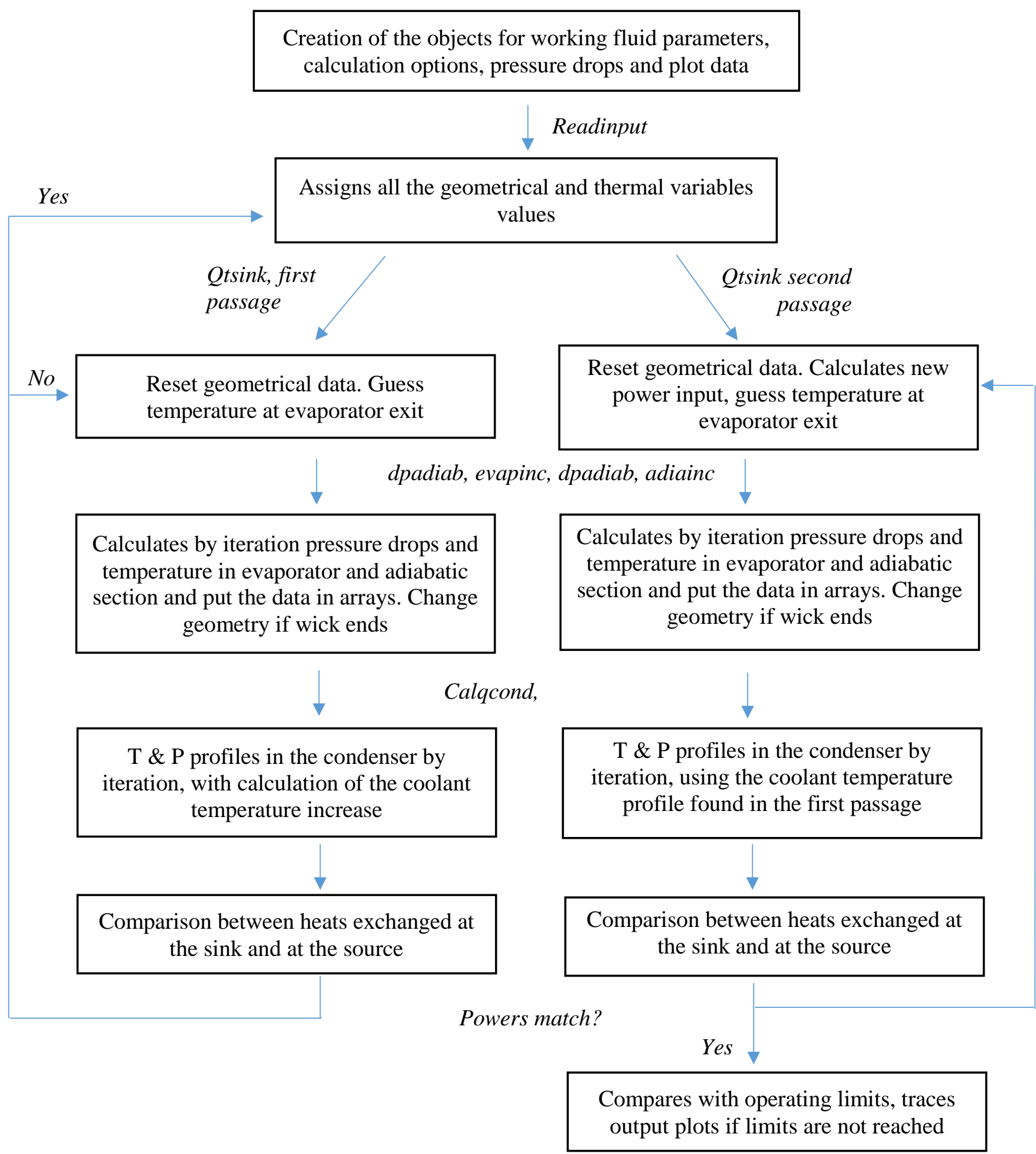

No 


\section{Annex 2: Input file format}

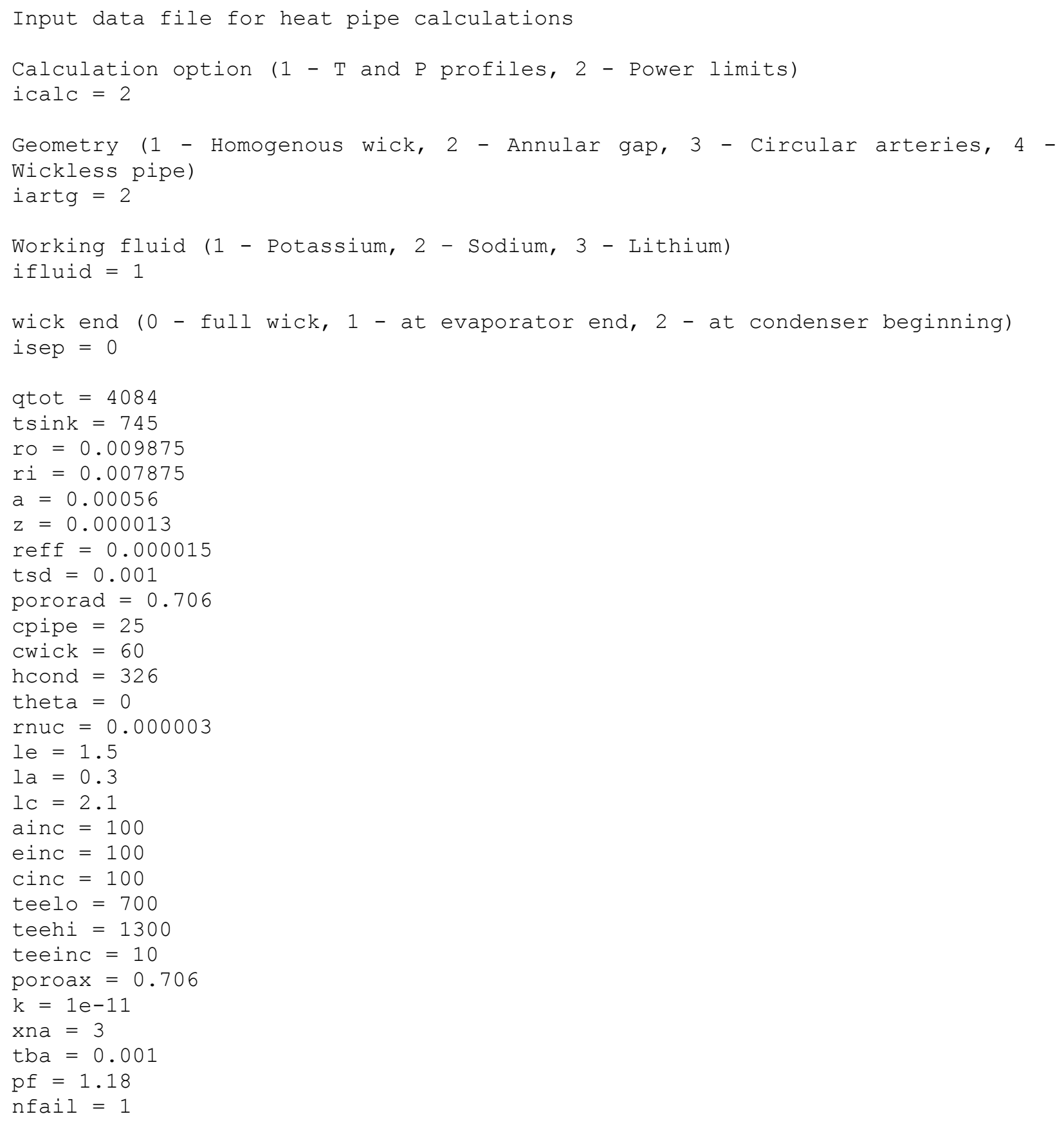




\section{Annex 3: Heat transfer with source, example of input file}

$$
\begin{aligned}
& \text { q } \\
& 59.8475 \\
& 68.3475 \\
& 76.3475 \\
& 83.8475 \\
& 90.8475 \\
& 97.3475 \\
& 103.3475 \\
& 108.8475 \\
& 113.8475 \\
& 118.3475 \\
& 122.3475 \\
& 125.8475 \\
& 128.8475 \\
& 131.3475 \\
& 133.3475 \\
& 134.8475 \\
& 135.8475 \\
& 136.3475 \\
& 139.5421 \\
& 142.4341 \\
& 136.3475 \\
& 135.8475 \\
& 134.8475 \\
& 133.3475 \\
& 131.3475 \\
& 130.0000 \\
& 128.8475 \\
& 125.8475 \\
& 122.3475 \\
& 118.3475 \\
& 113.8475 \\
& 108.8475 \\
& 103.3475 \\
& 97.3475 \\
& 90.8475 \\
& 83.8475 \\
& 76.3475 \\
& 68.3475 \\
& 59.8475 \\
& 50.8475 \\
& 41.3475 \\
& 31.3475 \\
& 20.8475
\end{aligned}
$$




\section{References}

T.J. Dickinson, "Performance Analysis of a Liquid Metal Heat Pipe Space Shuttle Experiment", M.S. thesis, Air Force Institute of Technology, 1996

M.S. El-Genk, J-M. P. Tournier, "Uses of Liquid Metal and Water Heat Pipes in Space Reactor Power Systems", Frontiers in Heat Pipes, 2011

B.I. Lee, S.H. Lee, "Manufacturing and Temperature Measurements of a Sodium Heat Pipe", KSME International Journal, Vol.15 No. 11, pp.1533-1540, 2001

R.E. Holtz, G.A. McLennan, E.R. Koehl, "On the Experimental Operation of a Sodium Heat Pipe", ANL85-61, Argonne National Laboratory, 1985

G.A. McLennan, “A Computer Code for the Simulation of Heat Pipe Operation”, ANL-98-108, Argonne National Laboratory, 1983

T.P. Cotter, “Theory of Heat Pipes”, LA-3246-MS, Los Alamos Scientific Laboratory, 1965

P.J. Brennan, E.J. Kroliczek, Heat Pipe Design Handbook, Prepared for NASA by B\&K Engineering Inc., pp. $12-50,1979$

K.A. Woloshun, M.A, Merrigan, E.D. Best, "HTPIPE: A Steady-State Heat Pipe Analysis Program”, LA11324-M, Los Alamos Scientific Laboratory, 1988

D.A. Reay, P.A. Kew, R.J. McGlen, Heat Pipes Theory, Design and Application, Sixth Edition, Elsevier, pp. $15-64,2014$

M.A. Merrigan, "Heat Pipe Technology Issues", $1^{\text {st }}$ Symposium on Space Nuclear Power Systems, LA-UR84-1238, Los Alamos National Laboratory, 1984

D.E. Glass, M.A. Merrigan, J.T. Sena, "Start Up of a Nb-1\%Zr Potassium Heat Pipe From the Frozen State”, Prepared for Langley Research Center, NASA, 1998

L.K. Tower, K.W. Baker, T.S. Marks, "NASA Lewis Steady State Heat Pipe Code Users Manual”, Lewis Research Center, NASA, 1992

W.S. Chang, "Porosity and Effective Thermal Conductivity of Wire Screens", Journal of Heat Transfer, Vol. 112, 1990

C. Tarau, Advanced Cooling Technologies Inc., personal communication, June 2017

A. Faghri, Heat Pipe Science and Technology, Second Edition, Global Digital Press, pp.77-256, 2016

J.J. Martin, R.S. Reid, "Sodium Based Heat Pipe Modules for Space Reactor Concepts: Stainless Steel SAFE-100 Core", Proceedings of International Congress on Advances in Nuclear Power Plants, Marshall Space Flight Center, NASA, 2004

S.W. Chi, "Heat Pipe Theory and Practice, A Sourcebook", McGraw Hill Book Company, 1976 
C.A. Busse, "Theory of the Ultimate Heat Transfer Limit of Cylindrical Heat Pipes", Journal of Heat Mass Transfer, Vol. 16, pp. 169-185, 1973

A Faghri, M.M. Chen, M. Morgan, "Heat Transfer in Two-Phased Closed Conventional and Concentric Annular Thermosyphons", ASME J. Heat Mass Transfer, Vol 111, pp. 611-618, 1989

P.R. Mcclure, D.I. Poston, V.R. Dasari, R.S. Reid, "Design of Megawatt Power Level Heat Pipe Reactors", LA-UR-15-28840, Los Alamos National Laboratory, 2015 